\title{
Linking structural and process-oriented models of plant growth
}

\author{
Development and test of the software NEXUS \\ as a multiple interface for \\ functional-structural models
}

\section{Dissertation}

Zur Erlangung des Doktorgrades

Der Fakultät für Forstwissenschaften und Waldökologie

Der Georg-August-Universität zu Göttingen

Vorgelegt von

Gustavo A. Anzola Jürgenson

Geboren in Valera, Venezuela

Göttingen, März 2002 
D7

Erster Gutachter: Prof. Dr. Dr. h.c. B. Sloboda

Zweiter Gutachter: Prof. Dr. W. Kurth

Dritter Gutachter: Prof. Dr. G. Gravenhorst

Tag der mündlichen Prüfung: Freitag, den 19.04.2002 


\section{ACKNOWLEDGEMENT}

I thank Prof. Dr. Dr. h. c. B. Sloboda, director of the Institute of Forest Biometry and applied Computer Sciences of the University of Göttingen, who supported me from the beginning and made the realisation of this project possible.

My special thanks to Prof. Dr. Winfried Kurth, for supporting, leading and motivating me though these last years, for proof-reading this thesis and for being a good friend.

I thank Dr. Jean Dauzat from CIRAD, France, for offering his knowledge and experience and providing valuable data and information indispensable for the project, and Dr. Jan Čermák, who collected and provided the sap flow data for the sampling site in Unterlüß.

I thank my co-workers at the Institute of Forest Biometry and applied Computer Sciences of the University of Göttingen, Dr. Michael Schulte for offering me his knowledge about plant physiology and for his inspiring questions, Helge Dzierzon and Ulrike Singer for their help with the programming and Olaf Oliefka for mapping the sampling trees and proof-reading part of this thesis. During my stage at the CIRAD in Montpellier, Jean Dauzat, Christophe Godin, Christophe Nouguier, Pascal Ferraro, Jean-François Barczi, François Houllier and Odile Chouillou helped me with their collaboration and friendship. Thank you!

The financial support for this project was provided by the DFG, the German Society of Research, and partially by the CIRAD, France, and by the Institute of Forest Biometry and applied Computer Sciences of the University of Göttingen. I thank them all very much. 



\section{LIST OF CONTENTS}

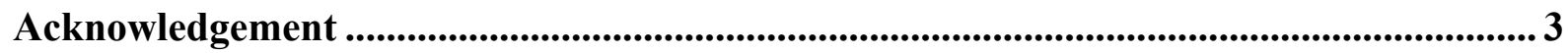

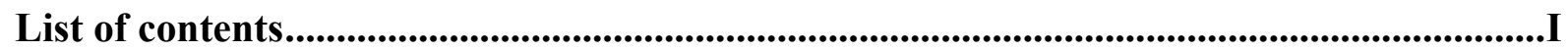

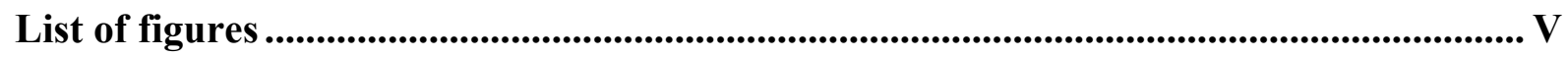

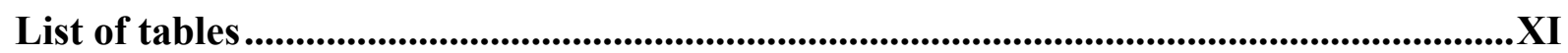

Abbreviations and symbols ................................................................................................ XII

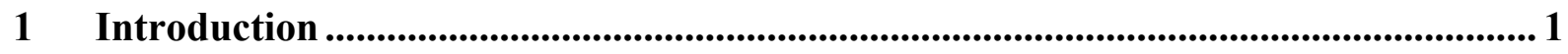

1.1 The development of plant models in the last years ........................................... 1

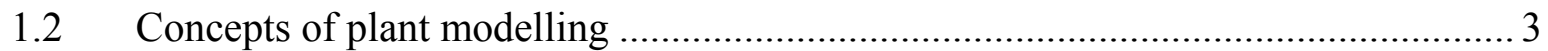

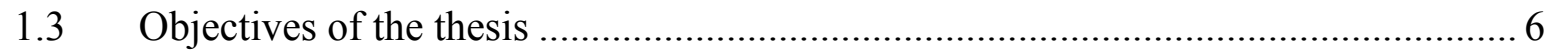

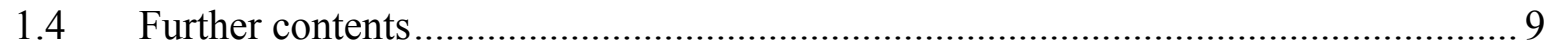

2 Plant modelling: State of the art ..................................................................................... 11 


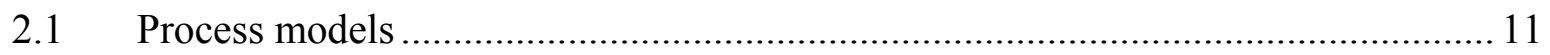

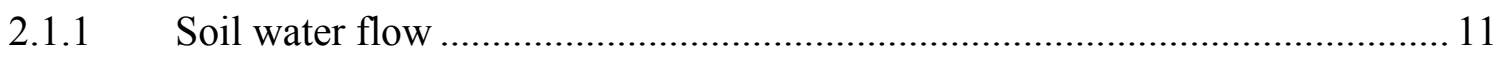

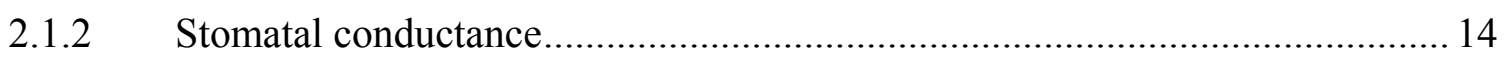

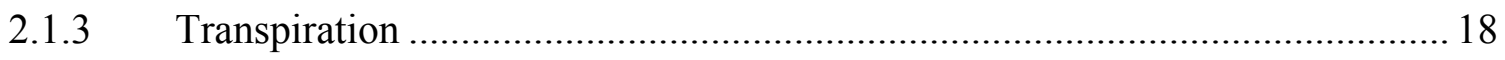

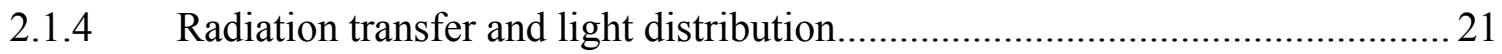

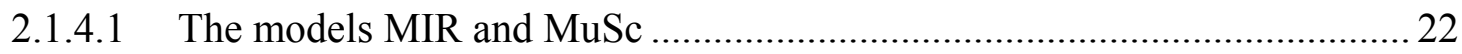

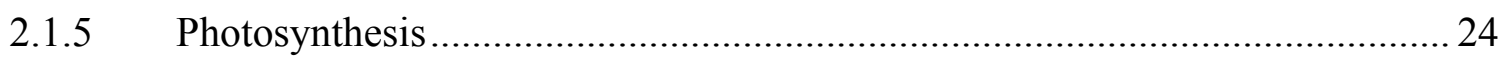

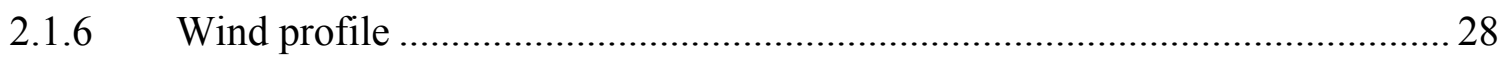

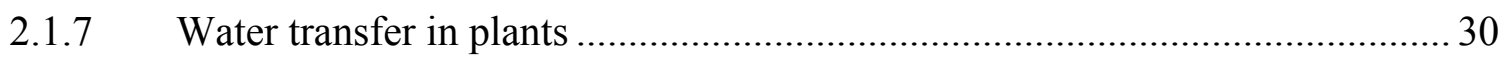

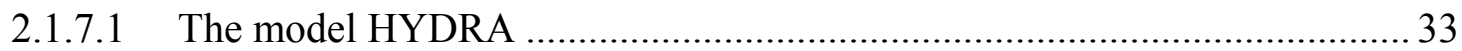

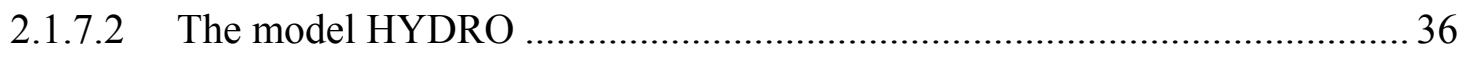

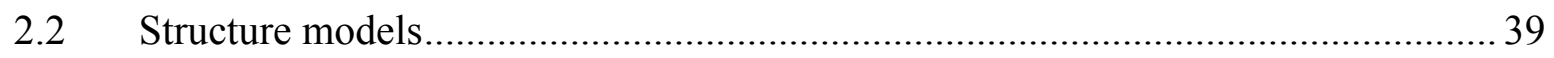

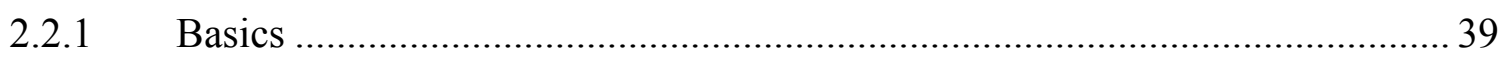

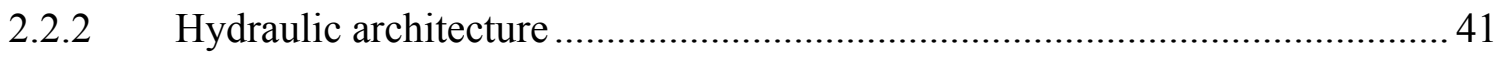

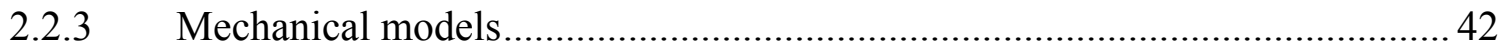

2.2.4 Forest models and the transition to functional-structural models .................. 43 
2.2.4.1 Single tree based models

2.2.4.2 Lindenmayer Systems and GROGRA

2.2.4.3 AMAP

2.3 Functional-structural models. 57

3 Material and methods.

3.1 Validation material. 67

3.1.1 Sampling plots 67

3.1.1.1 Costa Rica 67

3.1.1.2 Germany 68

3.1.2 Meteorological data. 70

3.1.3 Plant material. 71

3.2 Data formats. 76

3.3 Programming techniques.

4 The shell program NEXUS.

4.1 The Graphical User Interface (GUI) 92

4.2 Classes 


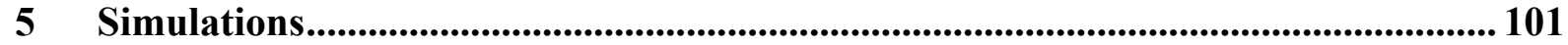

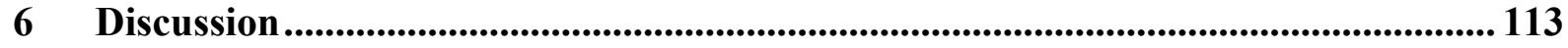

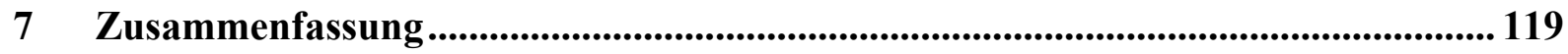

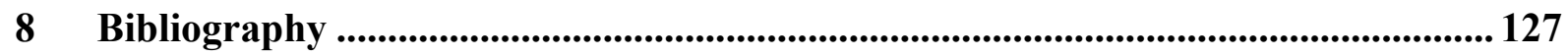

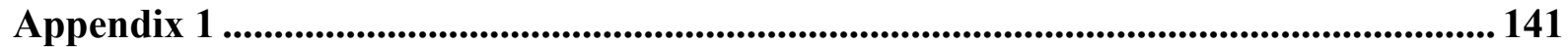

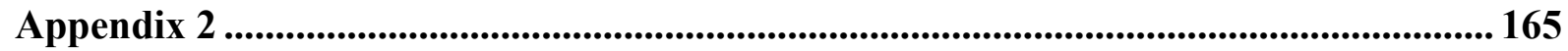

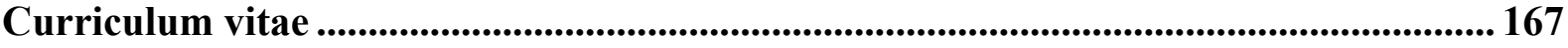




\section{LIST OF FIGURES}

Figure 1-1: Individual model interconnection. Each arrow represents a possible interface between two models. The dotted arrows represent interfaces already realised in previous projects for single programs. With this procedure a single interface must be developed for each new software tool, thus increasing the number of interfaces quadratically with each new module.

Figure 1-2: Model interconnection with a central co-ordination shell. Each module needs only one interface to the central program (NEXUS) that provides indirectly connections to all other modules. The number of necessary interfaces grows only linearly with the number of modules

Figure 1-3: General working scheme of NEXUS in the example of plant hydrology processes. The solid arrows represent the main data fluxes for the "core" and "peripheral" modules. The dotted arrows represent expanded information fluxes from "transition" modules

Figure 2-1: Diagram of a typical distribution of measurements of stomatal conductance $\left(g_{\text {sto }}\right)$ against an example variable $n$ as one of the variables in $g_{s t o}=g_{\max } \cdot f(P A R) \cdot f\left(R_{g}\right) \cdot f(\delta e) \cdot f(\Psi) \cdot f\left(C_{S}\right) \cdot \ldots$ The line demonstrates the approximation of the upper limit of observations to get $f(n)$, where all the other variables are supposed to be not limiting the stomatal conductance. While this approximation is made for each of the variables, the curve of each function does not necessarily match the curve shape shown above.

Figure 2-2: Typical light extinction curve according to Beer-Lambert's Law taking into account the leaf-mass density at different heights.

Figure 2-3: Turtle model for the sky hemisphere. The turtle is divided in 46 sectors. For each sector the incoming radiation is calculated. Cloud cover is calculated from the difference between global and extraterrestrial radiation.

Figure 2-4: Photosynthetic rate in dependence on light intensity (a), temperature (b) and air $\mathrm{CO}_{2}$ concentration

(c). The effects of temperature and $\mathrm{CO}_{2}$ concentration are greater by increasing light intensity. Temperature regulates enzyme reactions, and a higher $\mathrm{CO}_{2}$ concentration means a higher input. However, the whole process is principally regulated by the photochemical reactions, and thus by light intensity.

Figure 2-5: Characteristic wind profiles outside (a) and inside (b) a tree stand. For open fields and for the space above the canopy, the wind profile follows a logarithmic law. Inside the canopy, the wind velocity profile follows an exponential law. These formulas are valid under the assumption of a homogeneous canopy. For 
(a) is $z_{0}=0.13 \mathrm{~m}$ and $d=0 \mathrm{~m}$, and for (b) $z_{0}=2.6 \mathrm{~m}$ and $d=12.6 \mathrm{~m} . v_{0}=30 \mathrm{~m} / \mathrm{s}, h_{0}=30 \mathrm{~m}, \mathrm{hc}=20 \mathrm{~m}$ and the von Karmán constant $k=0.4$.

Figure 2-6: Diagrammatic representation of the simple pipe model of plant form (after SHINOZAKI ET AL., 1964). Diagram $\mathrm{C}$ shows the vertical distribution of leaves $(\Gamma(z))$ and branches $(C(z))$. A is the unit pipe, that can be united into a unit pipe system as the stand (B) or a single plant (D)

Figure 2-7: Diagrammatic representation of the pipe model of tree form, showing the successive accumulation of disused pipes in the trunk associated with the progress of tree growth (after SHINOZAKI ET AL., 1964) 32

Figure 2-8: Spatial discretisation for an unbranched part of the hydraulic network with variable cross sections. For the shaded region, mass conservation is assumed. The arrows indicate water flow and transpiration (after FRÜH, 1995).

Figure 2-9: Spatial discretisation scheme in the vicinity of a branching node. For the shaded cell, mass conservation is assumed. The arrows indicate water flows across the border and transpiration (after FRÜH, 1995).

Figure 2-10: Steady state water potential profiles generated by HYDRA. After selecting any number of nodes from the hydraulic map, HYDRA calculates the water potentials along the resulting flow paths. (a) shows the profile for a spruce tree simulated with GROGRA. (b) shows the profile for a young spruce tree from Solling mapped by ANZOLA (1998). After FRÜH AND KURTH (1999).

Figure 2-11: Function schema of the water flow model HYDRO from J. Dauzat. Ei is the evapotranspiration of the leaf element i used to calculate the water deficit $\Phi$ n of each segment $\mathrm{n}$. $\Psi \mathrm{j}$ is the hydraulic potential at point $\mathrm{j}$, where $\Psi 5$ is the potential at the root collar. $\Delta \mathrm{Vn}$ is the change of the water reserves, $\mathrm{Rn}$ the hydraulic resistance and $\mathrm{Cn}$ the hydraulic capacity of segment $\mathrm{n}$ (After RAPIDEL, 1995).

Figure 2-12: Architecture models proposed by Hallé and Oldeman. A-Holttum, B-Corner, C-Tomlinson, DChamberlain, E-Leeuwenberg, F-Fagerlind, G-Petit, H-Aubréville, I- Schoute, J-Nozeran, K-Massart (Abies, Ilex), L-Roux, M-Cook, N-Koriba, O-Rauh (Pinus, Acer), P-Prevost, Q-Scarrone (Aesculus), RAttims, S-Troll (Fagus, Tilia), T-Mangenot, U-Champagnat (Prunus, Rosa). After Richards, 1996. Not represented are the models McClure and Stone

Figure 2-13: Representation of a Picea sitchensis tree with the model of RENSHAW (1985). a) Visualisation of original data. b) Tree simulated with high variance. c) Tree simulated with low variance.

Figure 2-14: Structural root system simulated with the model of RENSHAW (1985): a) view from above, b) and c) lateral views.

Figure 2-15: Simulation of 14 branching systems (A-N) with the model of LÜCK ET AL. (1990) using different parameters. The systems from A to H correspond to the determinate growth of tomato (Lycopersicon spc.), with acrotonic branching. The systems I to $\mathrm{N}$ correspond to indeterminate growth.

Figure 2-16: a) Simulation of a single Abies sachalinensis tree at different ages. b) Simulation of a 35 years old stand after thinning. After SUZUKI ET AL. (1992).

Figure 2-17: Working scheme of an L-system. $\alpha$ is the initial string, $\sigma_{t}$ the string at time $t$, and $\mathrm{S}_{t}$ the corresponding geometrical structure. Horizontal arrows represent the application of generative rules, vertical arrows the geometrical interpretation. After KURTH, 1999.

Figure 2-18: Three levels of the formal description of plant structures. a) static geometrical description. b) Development of a structure over time as achieved by basic L-systems. c) Control of the development as 
achieved with stochastic or sensitive L-Systems. The arrows represent the application of generative rules (after KURTH, 1994).

Figure 2-19: Frequency distribution of number of internodes per growth unit at different branching orders and modalities in black cherry (Prunus avium). The axes of order 2 and 3 have pre-formed and neo-formed growth and thus a bimodal distribution. Axes of order 1 and short shoots have only pre-formed growth and thus a unimodal distribution. On the graphics, the horizontal axis is the internode number and the vertical axis the frequency (after DE REFFYE ET AL., 1991).

Figure 2-20: Distribution of branched and unbranched zones in a growth unit. The branching process is described by a Markov chain with the transition probabilities $\mathrm{P}_{1}$ (unbranched $\rightarrow$ unbranched) and $\mathrm{P}_{2}$ (branched $\rightarrow$ branched). Q is the global branching probability, and is independent from the distribution itself. With different parameters, diffuse and clustered distributions can be generated.

Figure 2-21: Plant growth according to the physiological gradient in a reference axis with five stages. The apical meristem changes gradually from one stage into the next. Lateral meristems can jump to a higher stage, and thus become physiologically older. (DE REFFYE ET AL., 1995a).

Figure 2-22: Representation of a plant with a tree graph coded by a string. The brackets in the string symbolise branching points (GODIN, 2000).

Figure 2-23: Concept of multi-scale tree graphs. A plant can be represented at different scales: a) axis, b) growth units, and c) internodes within a single multi-scale graph d) (GoDIN, 2000).

Figure 2-24: Encoding of plant structures with MTG in form of a string. Each scale is given by a slash followed by an indicator. /P is the plant scale, /U growth unit scale and /I internode scale. The symbol + means a branching point. Parameters at the end of a line belong to the last element of that line. In this case, growth unit $\mathrm{U} 1$ at the base of the plant has a length of 10 and a diameter of 5.9 and the growth unit $\mathrm{U} 1$ at the lateral branch has the values 7 and 3.5 respectively (GoDIN, 2000).

Figure 2-25: Three-dimensional representation of a Populus tree with ECOPHYS (HOST ET AL., 1990).

Figure 2-26: Celestial sphere model of CHIBA ET AL. (1994). Incoming radiation is calculated using the projected shadow or the leaf clusters on the sphere. From the lower hemisphere comes only diffuse radiation.

Figure 2-27: Examples of simulation results from CHIBA ET AL. (1994). Above, simulation of normal growth. Below simulation of the crown restoration after isolating one of the trees.

Figure 2-28: Representation of the voxelspace surrounding the canopy of a plant with MADEIRA. a) The plant in the voxelspace. b) The distribution of the leaf area among the voxels. b) Light reduction (after LIST AND KÜPPERS, 1998).

Figure 2-29: Simulation of a solitary tree with the light response model of TAKENAKA (1994) (above) and crosssectional view (below).

Figure 2-30: Calculated intercepted light with a hemispherical model (after KELLOMÄKI AND STRANDMAN, 1995).

Figure 2-31: Image of a simulated tree by Kellomäki and Strandman.

Figure 2-32: Simulation of growth in dependence on light. a) Tree adapted to grow in shade. b) Tree with increased light sensitivity. c) Effect of partial shadowing through an obstacle. (After www.metla.fi, 2002). 
Figure 2-33: Three isolated trees modelled with ALMIS after 150 time steps. The sun type has only sun leaves, the shade type only shade leaves and the adaptive type sun and shade leaves distributed according to the light environment.

Figure 3-1: Picture of the coffee stand in Turrialba, Costa Rica, made from the top of a $15 \mathrm{~m}$ high measurement tower situated in the centre of the stand (RAPIDEL, 1995).

Figure 3-2: Mixed stand in Unterlüß, Germany. Old growth stand, consisting mainly of Pinus sylvestris with mixed rejuvenation. View from west (above) and north (below).

Figure 3-3: Scheme of the sampling stand in Unterlüß and of some of the collected trees. The stand is fenced and very inhomogeneous. In the north, there are mostly old trees (Quercus and Pinus) with almost no rejuvenation. At the east, the old trees have been cut. The rest consists of some old trees, especially Pinus sylvestris, above a rich mixed rejuvenation, predominantly Quercus spc. Besides the five sampling trees from the middle represented here, two other exemplars were collected at the eastern part of the stand. Besides the sampling trees, significant neighbours and their respective species have been represented.

Figure 3-4: Meteorological data from Turrialba, Costa Rica, measured at a sunny day (april $13^{\text {th }}$ ), and at a coudy day (april, $27^{\text {th }}$ ). The data for the night from april $26^{\text {th }}$ to $27^{\text {th }}$ has been ommited (Data provided by Jean Dauzat, CIRAD, France).

Figure 3-5: Global radiation, air temperature and relative humidity values measured from June $27^{\text {th }}$ till July $6^{\text {th }}$ in Unterlüß, Germany. All values were measured at a height of 36 m every 30 minutes.

Figure 3-6: Representation of a Coffee plant from Costa Rica generated with the tool "Landmaker" from the AMAP software.

Figure 3-7: Example of a mapped structure and its representation with GROGRA.

Figure 3-8: Sampling trees ejk (left) and ejf(right). Tree ejk grows below a pine tree with a $d b h$ of $30 \mathrm{~cm}$ and a height of ca. $25 \mathrm{~m}$. This tree was measured in situ. Tree ejf grows within a rejuvenation group and was measured destructively after sampling.

Figure 3-9: Representation of the trees ejk (left) and ejf (right) with GROGRA.

Figure 3-10: Representation of the trees ejk (left) and ejf (right) with AMAP.

Figure 3-11: Measured sap flow for the tree ejk, ejh, ejf, ejg (below) and eji (above). On tree eji two separate sap flows were measured, once on the collar (eji low) and once on a branch in the upper part of the crown (eji high).

Figure 3-12: Replication modes used in scu-files. In option 0, P2 is ignored because it is outside the used scene. In option 2, P2 is still ignored, but P1 is duplicated to create the surrounding environment. In option 3, P2 is used as part of the surrounding environment.

Figure 3-13: Example for class hierarchy and inheritance. Organ is the base class for Internode, Foliage and Bud. Leaf and Needle are derived from Foliage. A Needle object would have a curvature value, a maximal age (by itself), transpiration and photosynthesis rates (from Foliage), and dry mass and physiological age (from Organ).

Figure 3-14: Screenshots from WGROGRA (left) and XGROGRA for SGI IRIX systems (right). The pictures show the same menu in both versions with modified gray tones. 
Figure 3-15: Screeshot of QMIR with its visualisation tool. The picture represents tree ejk viewed from sector 10 of the "turtle". The resolution of the picture depends od the number of pixels selected in the configuration file. Original colors have been changed for better reproduction.

Figure 3-16: Screenshot of QNEXUS displaying the dialog for starting GROGRA. Every command line parameter can be selected in this dialog. With a special option, GROGRA runs interconnected with NEXUS via socket.

Figure 4-1: Representation of the tree structure in different formats. a) The standard model in GROGRA represents the structure at GU level. b) In AMAP, internodes and nodes are included in the structure files. c) In the pbg file from HYDRA, the structure is changed to meet the requirements for the numerical model. NEXUS keeps track of the relation between structures.

Figure 4-2: Generic graphical user interface (GUI) in the NEXUS project. 1) Project window. 2) Editor window.

3) Output window. 4) Menu and tool bars.

Figure 4-3: Parameter dialog of QMIR. The parameters can be selected directly in the command line. This dialog is also used in NEXUS to create the command line of MIR.

Figure 4-4: Picture generated by QMIR and viewed with the tool ViewPix. QMIR calculates the pixels that are hit by a light ray coming from an specific direction (ray tracing method). The output can follow different options. Here each plant has a different colour.

Figure 4-5: AMAP scene used to generate the picture in Figure 4-4. 96

$\begin{array}{lr}\text { Figure 5-1: Scene for ejc. } & 101\end{array}$

Figure 5-2: Scene for ejd. $r$

Figure 5-3: Scene for ejf. $r$

$\begin{array}{lr}\text { Figure 5-4: Scene for ejg. } & 103\end{array}$

$\begin{array}{lr}\text { Figure 5-5: Scene for ejh. } & 103\end{array}$

$\begin{array}{lr}\text { Figure 5-6: Scene for ejk. } & 104\end{array}$

Figure 5-7: Evolution of calculated transpiration values of a coffee plant according to the meteorological data from Costa Rica provided by Dauzat (see 3.1.2).

Figure 5-8: Comparison of measured and calculated leaf temperatures. The black symbols represent the original data, the white symbols represent the modified data after the multiplication of the boundary layer resistance (BLR) by 2 (After RAPIDEL, 1995).

Figure 5-9: Results of the first simulation of the coffee plant and tree ejk using meteorological data from Costa Rica. The diagram shows the assimilation for April 27, 1994.

Figure 5-10: Sap flow of all sampling trees in relation to meteorological data: Global radiation, relative air humidity, air temperature and air vapour pressure deficit. The lower flow rates correspond to the smaller trees, ejk, ejk and ejh. The upper part of the scatter-plot correspond to the larger trees ejf and eji. The flow rate depends upon the respiration surface, and thus upon the number of leaves, and overall conductance.

Figure 5-11: Measured and calculated sap flows for the trees ejk and ejf. Calculated values tend to underestimate the measured values by up to $20 \%$ divergence.

Figure 5-12: Assimilation of trees ejk and ejf.

Figure 5-13: The assimilation of the same tree changes drastically between solitary and competition growth. 109 
Figure 5-14: Spruce trees generated with the L-system epi2cdi2.ssy, written by Kurth, after 3 years (left), 5 years (middle) and 7 years (right). The L-system reads data from a table which is generated by NEXUS. Flow of data from radiation or hydraulic calculations is not yet realised.

Figure 5-15: Scheme of the interconnection NEXUS-GROGRA with a socket for data transfer. GROGRA waits for a signal from NEXUS to start the growth simulation using the generated values, and sends a signal back when the simulation is finished. NEXUS calculates new values using other models like MIR, HYDRO or HYDRA and signalises GROGRA to continue with the growth simulation. 


\section{LIST OF TABLES}

Table 3-1: Example for the description of a plant structure using dtd (digitised tree data) format. The columns mean: identifier, length [mm], mother segment (\#\# is the base segment), location on the mother branch [mm] (V means the same axis), branching direction (+ right, - left), branching angle, diameter [mm], colour and possible attributes.

Table 3-2: Plant models to be interfaced with each other, together with their respective data file formats. The basic structure files describe the main part of the models. Advanced structure files, on the other hand, are expansions to the original formats based on higher mathematical concepts (formal grammars, graph theory). Supplementary data describe the general environment and framework of the simulated scenarios. 


\title{
ABBREVIATIONS AND SYMBOLS
}

\author{
$\Psi \quad$ Matrix potential of a porous medium [ $\mathrm{MPa}$ ] \\ $\theta \quad$ Water content in $\%$ \\ $\Phi \quad$ Water flow [ $\mathrm{kg} \mathrm{s}-1]$ \\ Se Water vapour pressure deficit $\left[\mathrm{mPA} \mathrm{Pa}{ }^{-1} ; \mathrm{Pa} \mathrm{kPa}^{-1} ; \mathrm{mmol} \mathrm{mol}^{-1}\right]$ \\ ol Leaf specific activity [ $\mathrm{g} \mathrm{C} \mathrm{g-1;g} \mathrm{C} \mathrm{m-2} \mathrm{]}$ \\ A Assimilation [ $\mathrm{mg} \mathrm{CO} 2 \mathrm{~h}-1$ ] \\ AMAP Atelier de Modelisation de l'Architecture des Plantes \\ AML AMAP Modelling Language \\ C Hydraulic capacity [ $\mathrm{kg} \mathrm{MPa} \mathrm{-1} \mathrm{]}$ \\ $\mathrm{Ci} \quad \mathrm{CO} 2$ concentration in the intracellular air $\left[\mathrm{cm}^{3} \mathrm{CO} 2 / \mathrm{m}^{3}\right]$
}

CIRAD Centre de Coopération Internationale en Recherche Agronomique pour le Développement

Cs $\quad \mathrm{CO} 2$ concentration on the leaf surface $\left[\mathrm{cm}^{3} \mathrm{CO} 2 / \mathrm{m}^{3}\right]$

E Evapotranspiration $[\mathrm{kg} \mathrm{MPa}-1]$

g Leaf conductance $\left[\mathrm{mmol} \mathrm{m}^{-2} \mathrm{~s}^{-1}\right]$

g gravitational acceleration $\left(=9.81 \mathrm{~m} \mathrm{~s}^{-2}\right)$

GROGRA GROwth GRAmar Interpreter

$\mathrm{g}_{\text {sto }} \quad$ Stomatal conductance $\left[\mathrm{cm} / \mathrm{s} ; \mathrm{mmol} \mathrm{m}^{-2} \mathrm{~s}^{-1}\right]$

GUI Graphical User Interface

$h \quad$ Height [ $\mathrm{m}$ ]

$\mathrm{H} \quad$ Hydraulic potential [ $\mathrm{m} \mathrm{PA} ; \mathrm{m}^{2} / \mathrm{s}^{2}$ ]

$\mathrm{k} \quad$ Hydraulic conductivity [ $\mathrm{m} / \mathrm{s}$ ]

$\mathrm{k} \quad$ Von Karmán constant $=0.4$

LAI Leaf Area Index 


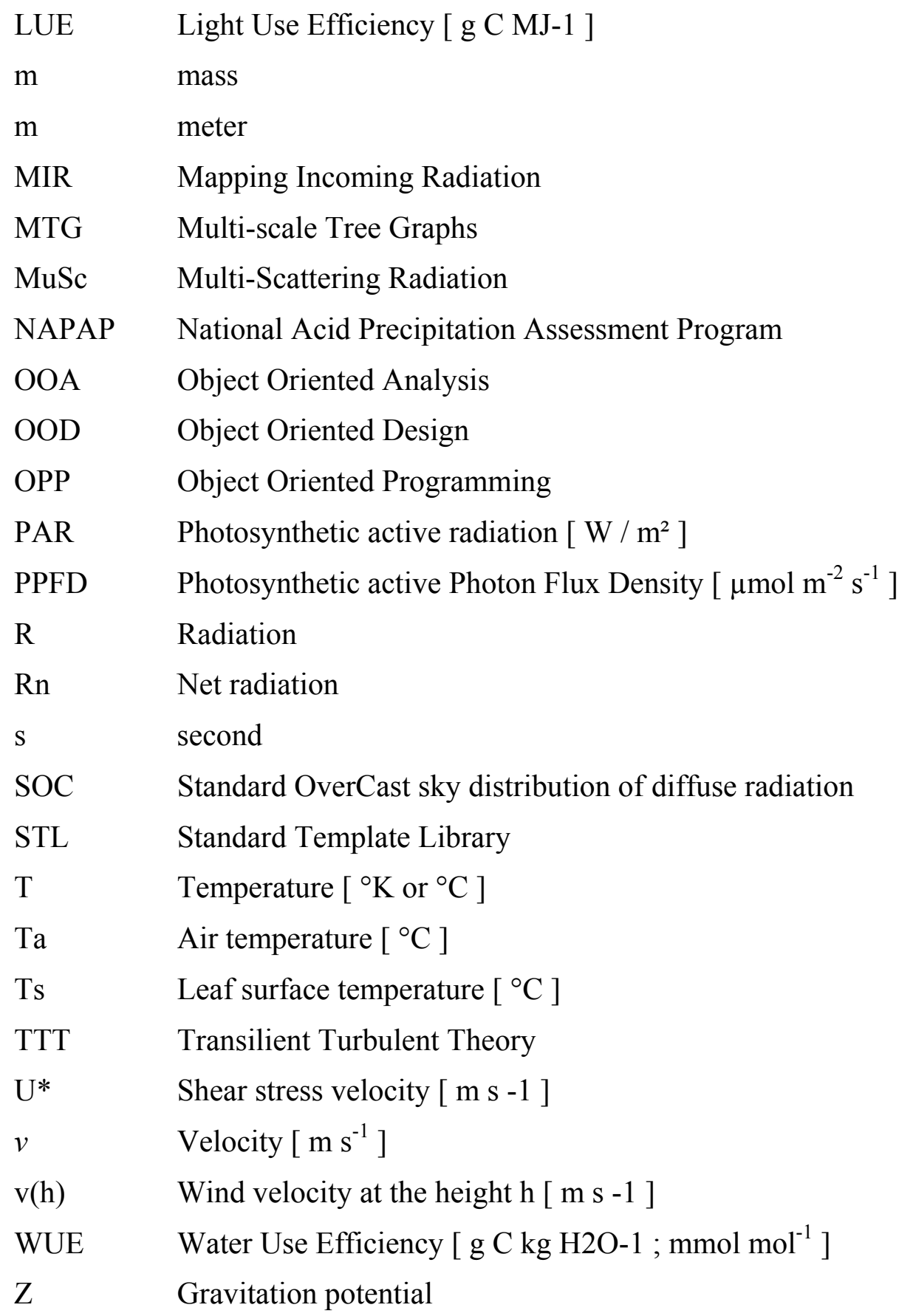





\section{INTRODUCTION}

\subsection{The development of plant models in the last years}

In the last decades the modelling of plant growth has become an inherent part of the theoretical and practical decision making processes in forestry and agronomy. A vast number of empirical models, based on long term observations, have been developed and used as standard methods of the classical forestry, namely yield tables and stand height curves. However, these models are no longer able to describe or prognosticate accurately the behaviour and reactions of trees and tree stands to their environment, because they do not consider the rapidly changing environmental conditions of our time. Global meteorological changes, pollution and new methods of forest management build a new scope of parameters and relationships that have an influence on the growth of plants.

The original question formulation for forest models was focused on the general changes of productivity and wood quality and on the measurement of these changes. In the last 20 years, the discussion about the impact of environmental change on plant growth was initially marked by the phenomenon of forest die-back (Waldsterben). The direct effects on the plant phenotype became a measurement tool for the condition of trees and the environmental impact on their growth (UlRICH, 1981; ROLOFF, 1984; GRUBER, 1987). In addition, due to different ecological and economical factors, new methods of forest management have been developed and used in the last years. These are mainly aiming at resource sustainability and natural stand structures (e.g. LÖWE program, ОтTO, 1989, 1991; NIEDERSÄCHSISCHE LANDESREGIERUNG, 1992), creating mixed stands containing both different species and trees in different ages. This sustainable and ecological silviculture has to be taken into account by 
modern models, as it represents a significant difference to the growth conditions in older stands.

All these processes and needs led to the development of models able to describe single plants and stand structures at different scales to better represent and study the relationships between plants, and between plants and their environment in a three-dimensional scope. On the other hand, the interest and the wish to better understand the effects of the flow, interchange and concentration of different substances (nutrients, pollutants, water, energy, etc) also led to the development of models based on physical, chemical and physiological processes, such as photosynthesis, transpiration, respiration and water and nutrient uptake. Such models generally use simplified structures that serve as spatial frame for the processes. These structures consist of abstract spatial compartments, such as whole plants (BASSOW ET AL., 1990), crown (CONSTANTIN, 1993; IBrom, 1993), stem (Brough ET AL., 1986; ČERMÁK ET AL, 1976) and soil (BöHM, 1979; IstoK, 1989, LAFOLIE ET AL., 1991; SCHMIDT ET AL., 1995; MORGEnSTERn, 1996).

Although the interest of forestry is centred on tree and stand models, many of the plant models used in forestry have their origins in other research fields. KURTH (1999) divides the different modelling approaches into seven major schools:

I. The French school, which is concerned with the description of tree architecture (Hallé And Oldeman, 1970; Hallé ET AL., 1978; Hallé, 1986) as the result of both endogenous and exogenous processes, and later with the development of procedures and their practical implementation for the modelling of agrarian plants (DE REFFYE).

II. The theoretical biology, which provides important basic principles for the modern modelling of plants. These are for example the modular structure of plants, the development of the morphology as result of casual connections with different processes, and the optimisation of resources uptake which is related with the production of optimal structures.

III. The theoretical computer sciences and mathematics. From this field come most of the mathematical formalisms of modern plant modelling. 
IV. Computer aided graphics. This field is relatively new, and was originally centred on the production of optically attractive structures which had not always correct botanical background. However, the realistic visualisation of simulation results is today an important part of plant modelling. This field provided important insights in relation to spatial discretisation of data and for the optimisation of algorithms.

V. Forestry and forest ecology. As described before in this thesis, this research field is concerned with the prognosis of forest development under modern silviculture methods, and with the modelling of single trees.

VI. Bioclimatology and tree physics. This research field includes the modelling of photosynthesis in heterogeneous tree stands, the deposition of air pollutants and nutrients and their filtering through the canopy, and the description of the mechanical and hydraulic characteristics of crowns.

VII. Entomology. This field uses plant structures to represent and to describe the habitat of some insects and to study the effects of feeding on plant structures.

All these schools can not longer be considered individually, but rather as a network of ideas which can be combined to conceive better models that can satisfy the requirements and provide answers to actual questions of plant modelling.

\subsection{Concepts of plant modelling}

At this point it is necessary to clearly define a model. A model is reproduction of the reality with abstraction of redundant details. A model permits us to discern, comprehend and handle information without the knowledge about the whole background. The complexity of models is highly variable, depending on the degree of abstraction and naturally on the complexity of the part of the reality that it describes. The simplest models are mainly descriptive. A map provides us with different information about a landscape depending on its scale and on the number of details included. The more information we try to reproduce with the map, the more it becomes complex and difficult to read. A more complex form of description is for example, a chemical formula for a reaction, or a mathematical formula that describes a given set of 
data. However, to satisfy the needs and questions of modern silviculture, it is necessary to go further. The description of complex dynamic processes in organisms (trees) or ecosystems (stands) is obtained through simulation, i.e. the virtual reproduction of the reality at a structural-functional level using a model. Simulation models serve to describe, analyse and validate theoretical and empirical data that led to the model formulation, and to generate prognoses under different (plausible) scenarios. The simulation models are thus a powerful research and decision making tool for the practice. However, it is important always to remember that a simulation is only a possible picture of the reality using a given set of boundary conditions and not the reality itself. Unfortunately, there is a tendency to overvalue the results of simulations.

A vast number of plant models have been developed using different approaches, depending on the goals of the respective project. In general, the modelling approaches for plant growth can be classified in three main categories, whose boundaries, however, can be vague. Thus, an exact assignation for each model to a category is not always possible without ambiguity.

First, process oriented models such as photosynthesis and C-allocation models. These models are based on the physical interaction between ecological compartments that can be defined on different time and space scales. One characteristic of this kind of models is that the definition of the spatial compartments used to delimit the process can be very abstract. These models often consist of a couple of mathematical formulas and rules that describe the given process. This is given as the description of a state or a dynamic, especially equilibrium condition, threshold values, flow rates, uptake/loss rates, etc, or a combination of them (BASSOW ET AL. 1990; THORNLEY, 1976; BOSSEL, 1994).

Second, structural models based on the morphological architecture of the plant. Thus, these models are devoted to the observed growth topology of the plants without taking in consideration the interaction with the environment and the internal physiological processes of the plant. In this regard we have to distinguish between plant topology and plant morphology. The term "plant topology" describes the relative relationship between the different elements of the plant - especially nodes and internodes - and their arrangement in space, while the term "plant morphology" describes the general shape and form of the plant and its elements, along with their biological significance. Morphological models are generally based on empirical 
botanical observation with abstraction from the physiological processes that control plant growth. Examples of this group are crown architecture models (HALLÉ AND OLDEMAN, 1970; HALlÉ ET AL., 1978; KRANIGK, 1995) and basic Lindenmayer systems (PRUSINKIEWICZ, 1987; Prusinkiewicz et AL., 1988; Prusinkiewicz, 1994; Kurth, 1994; Kurth, 1997; Kurth, 1999). These models try to reproduce the observed or perceived reality without having to reproduce the processes or environmental conditions that led to a given state. A representative example for such models is the description of the crown morphology using length/diameter relationships to create new branches, and the general approach of the pipe model (DA VINCI, after RICHTER, 1970; SHINOZAKI ET AL., 1964) to simulate the secondary diameter growth.

At last, a new group of models has emerged, that increasingly unifies process modelling with more and more complex structural models (KELLOMÄKI AND KURTTIO, 1991; KELLOMÄKI And Strandman, 1995; KellomäKI AND IKONEN, 1996; PertTUNEN ET AL., 1996; PertTUnen ET AL., 1998; SiEVÄNEN ET AL. 1995) where the structure is more than only the physical frame of the processes. Process and structure interact with each other and are thus interdependent. Thus, a direct description of plant growth in dependence on its physiological processes is made possible. This third category comprises the structural-functional models based on the reconstruction of the topological and/or morphological architecture of the plant, taking into account the dynamics of specific physiological and mechanical processes as well as the interaction of the plant with the environment and other plants (light, water, nutrients, and competition). In this context, we can also mention the model ECOPHYS (HoST ET AL., 1990), the AMAPpara software from CIRAD, France (DE REFFYE ET AL., 1995a; DE REFFYE ET AL., 1995b) and the advanced formalisms of the already mentioned L-systems.

For this category of models the differentiation in topological and morphological architecture is also important, because there exist models devoted to the reproduction and representation of tree stands, where the fine structure of single plants is ignored and only "raw" morphological elements are used (i.e. cones, spheres or ellipsoids are used to represent single tree crowns, Pfreundt And Sloboda, 1996; Kahn And Pretzsch, 1997; Pretzsch, 1990a, Pretzsch, 1990b; Pretzsch, 1992a; PretzsCH, 1992b). 


\subsection{Objectives of the thesis}

As shown above, the vast amplitude of models is the result of the separated and individual development of approaches in the different research branches of botany, forestry and agronomy. This has made it difficult to warrant the exchange of information between existing models. As a result, model outputs and results are often difficult or impossible to compare, even by very similar question formulation and model development.

At this time the actual project from which this thesis has originated includes five categories of models or software to be interconnected. First, the growth engines AMAP (DE REFFYE ET AL., 1995a, 1995b; BARCZI ET AL., 1997) and GROGRA (KURTH, 1994, 1999), i.e. the software for the simulation of growth and for the representation and visualisation of the geometrical and topological architecture of the plant and thus its morphology. Second, the models for internal hydrological processes and water flow in the plant itself, especially the model HYDRA by Thomas FRÜH (1995; FRÜH AND KURTH, 1999). Third, models that describe the microenvironment in the canopy layer, especially light and radiation balances, such as MIR/MuSc by J. Dauzat (Dauzat And Hautecoeur, 1991; Dauzat, 1993; DauZat, 1994; DAUZAT AND EROY, 1997; DAUZAT AND RAPIDEL, 1997; RAPIDEL ET AL., 1999). Fourth, models of root growth and behaviour. And fifth, models for the soil environment, including water flow, e.g. SilVlow (SCHMIDT ET AL., 1995), and nutrients. A common way of model interconnection is the creation of single interfaces between two modules, as already realised by the software AIR by D. LANWERT (LANWERT 1997; LANWERT ET AL., 1998) to interconnect HYDRA and AMAP, and by the model HYDRA (FRÜH, 1995), that offers a simple interface to the model SilVlow (SCHMIDT ET AL., 1995). The growth engines AMAP and GROGRA provide both a range of interfaces to other models. As shown in Figure 1-1, this method of model interconnection would lead to a quadratically growing number of interfaces, and thus it would also lead to the same problem as described above. There would be many different transcription tools that act individually and thus ineffectively, as the information exchange with other modules is not warranted or is only possible through manual corrections and single conversion.

It is thus necessary to create a generic tool, able to co-ordinate the interaction of these models. However, the exchange of information is often only possible in a static and unidirectional way. That is to say, the results of a model are integrated in other models and supply the 
general conditions for further processes, e.g. the architecture of the crown as framework for light interception. Another problem results from the large differences in temporal and spatial scales between models, due to their different objectives and methods. The co-ordination of time steps and the adjustment of spatial information is an important part of the model interconnection.
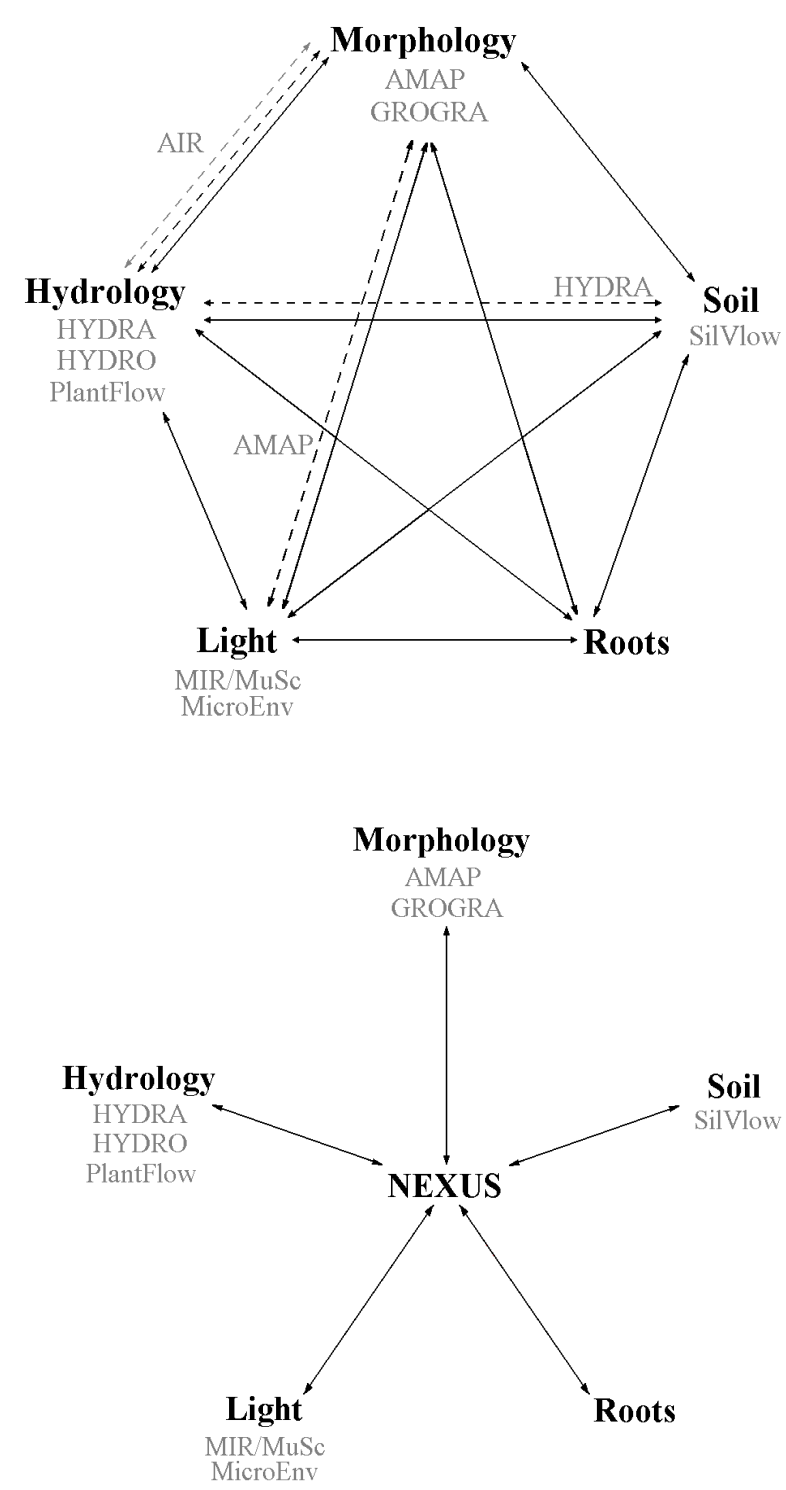

Figure 1-1: Individual model interconnection. Each arrow represents a possible interface between two models. The dotted arrows represent interfaces already realised in previous projects for single programs. With this procedure a single interface must be developed for each new software tool, thus increasing the number of interfaces quadratically with each new module.
Figure 1-2: Model interconnection with a central co-ordination shell. Each module needs only one interface to the central program (NEXUS) that provides indirectly connections to all other modules. The number of necessary interfaces grows only linearly with the number of modules

Main objective of this project is to create a software shell, called NEXUS that facilitates or even makes possible the interconnection of different models. For the development of the software some basic principles had to be defined. First, the software should be portable to different platforms, so that it works under different operation systems. This is necessary 
because models have been developed under different systems, especially Windows and UNIX. Second, the shell should have a modular architecture, so that the integration of new models at some indefinite future time (as new modules) is warranted. And third, the maintainability, portability and use of the software should be warranted through an exhaustive documentation. Figure 1-2 shows the schematic structure for a central interface that makes the data exchange between the modules more effective and manageable.

For the realisation of the model interconnection the co-operation between different work groups is very important. At present, the main co-operative work relevant for this study takes place between the following workgroups: the workgroup of W. Kurth at the Institut für Forstliche Biometrie und Informatik at the University of Göttingen, Germany, the workgroup of F. Houllier at the CIRAD (Centre de Coopération Internationale en Recherche Agronomique pour le Développement), Montpellier, France, and the work group of R. Sievänen at the METLA (Finnish Forest Research Institute), Vantaa, Finland. This cooperation includes the exchange of information, data and software, as much as collecting new data for validation of the model interaction. For this reason, the long-term development of the NEXUS shell will include five main groups of modules: First, the growth engines AMAP (CIRAD, France) and GROGRA (University of Göttingen, Germany), i.e. the software for the simulation of growth and for the representation and visualisation of the geometrical and topological architecture of the plant and thus its morphology. Second, the models for internal hydrological processes and water flow in the plant itself, especially the model HYDRA by T. Früh (University of Göttingen), and HYDRO by J. Dauzat (CIRAD, France). Third, models that describe the microenvironment in the crown layer, especially light and radiation balance. The modules include the programs MIR and MuSc by J. Dauzat (CIRAD, France). Fourth, models of root growth and behaviour (not yet included). And fifth, models for the soil environment, including water flow, e.g. SilVlow by C. Blendinger (Bonn), and nutrients. Figure 1-3 shows a general working scheme of NEXUS in the example of plant hydrology processes. The solid arrows represent the main process and information flow between the modules. They represent the data transfer in NEXUS and the process interfaces to coupled modules, e.g. Growth Engines and radiation models. The dotted arrows represent the information flow needed as model input, which can be produced by other external models. 
A similar project had been aimed at by the NAPAP (National Acid Precipitation Assessment Program, USA) to simulate single trees and tree stands (KIESTER ET AL., 1990). The project contained different models at different spatial and temporal scales, which combine numerous modelling approaches. For single trees, a meta-model called Simple Whole Tree had been designed. It contained five modules: NEEDLES, which describes needle physiology; BRANCH/FOLIAGE, which describes branch morphology and physiology; TRANS, which simulates the carbohydrate transport in phloem; ROOTS/SOIL, for the interaction of roots and the soil matrix; and WHORL, which describes the competition behaviour of the crown. The modules in turn are made of different structure-function models, with a focus on C- and $\mathrm{N}$-allocation and on transfer processes. These models were parameterised for Pinus taeda L. and Picea rubens Sarg. For whole stands, the model MAESTRO was used to calculate the radiation balance and the carbon gain through photosynthesis, and $\mathrm{NuCM}$ was used to simulate the cycling of nutrients in forested ecosystems. The single models are very complex and detailed, and have been individually validated. However, the final model integration into the proposed meta-model does not seem to have been achieved.

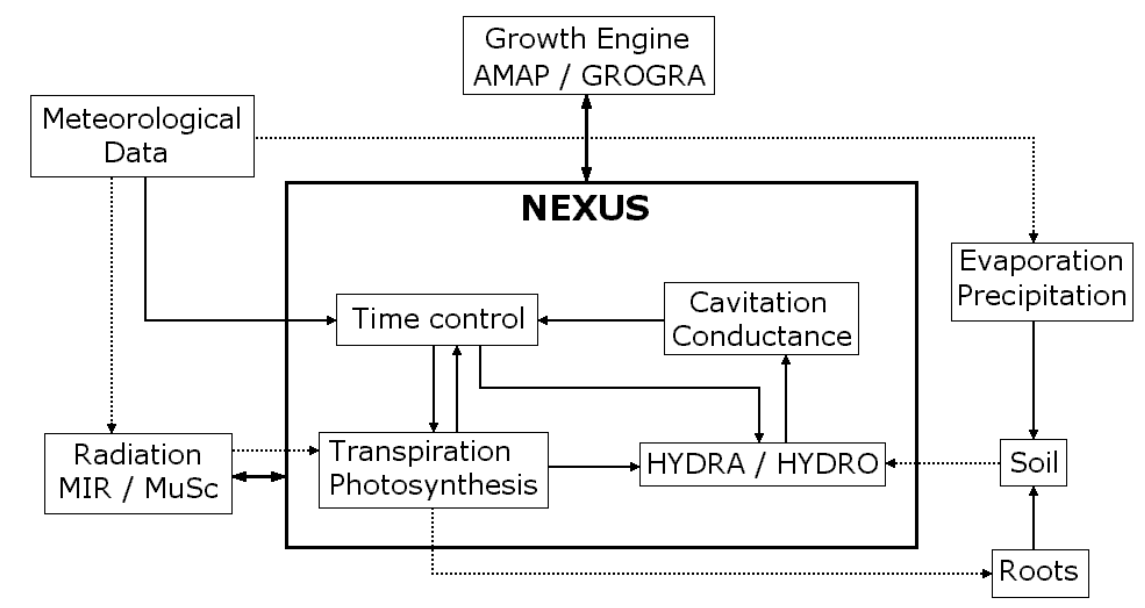

Figure 1-3: General working scheme of NEXUS in the example of plant hydrology processes. The solid arrows represent the main data fluxes for the "core" and "peripheral" modules. The dotted arrows represent expanded information fluxes from "transition" modules

\subsection{Further contents}

Chapter 2 of this thesis describes the most important modelling approaches in each of the three categories of plant models. The model description includes the model hypothesis, the input and output data and their format, and the description of how it interacts with other models, respectively how these have been included in existing models. 
Chapter 3 describes the programming techniques used to create the shell, especially object oriented programming (OOP). Further, the measurement methods and experimental sites used to get the validation material for the model interconnection as well as the methods used to validate it are described in this chapter as well.

In chapter 4, the shell software NEXUS is described in a more detailed fashion together with some additional programs used to create the interfaces.

In chapter 5 the validation and comparison of each used model takes place considering results obtained with and without the interconnection of the different models.

Chapter 6 is dedicated to the evaluation of the results and of the utility and benefits as much as of the drawbacks of the interconnection of the given models by means of a general shell. 


\section{Plant modelling: State of The aRT}

Following the classification presented above, we describe briefly some of the most relevant models, altogether with their utility, limitations, portability and connectivity with other models.

\subsection{Process models}

There exists a series of models for physical and physiological processes that have been already tried and tested, and that build the basics of most of the more recent process and structure-process models. In this context we concentrate on model approaches for soil water flow, stomatal conductance, transpiration, photosynthesis, radiation transfer, vertical wind velocity profiles and water flow in plants.

\subsubsection{Soil water flow}

The most important basic models for water flow rest upon the principle of the hydraulic potential $h$ that can be represented as follows under simplification of the physical characteristics of water

$$
H=\Psi+z
$$

where $\Psi$ is the matrix potential of the system and $z$ the gravitation potential. In some literature the formula 2-1 is also given as $H=h+z$. The units for the potential depend on the reference value used. Generally, the energy can be related to a mass, a volume or a weight of water, implying as resulting potential unit length ${ }^{2} / \mathrm{time}^{2}$, pressure and length units respectively. This 
can be illustrated by means of the gravitation potential, defined as $m \cdot g \cdot l$, where $m$ is the mass $(\mathrm{kg}), g$ the gravity acceleration $\left(\mathrm{m} / \mathrm{s}^{2}\right)$ and $l$ the height or length of the capillary $(\mathrm{m})$. In reference to the mass, we get $m \cdot g \cdot l / m=g \cdot$, thus $\mathrm{m}^{2} / \mathrm{s}^{2}$ as unit. In reference to the volume, we get $m \cdot g \cdot l / V=m \cdot g / l^{2}$, thus Pa as unit. And in reference to the weight, we get $m \cdot g \cdot l / m \cdot g=l$, thus $\mathrm{m}$ as unit. In the soil hydrology the most common units used are length units, especially meter.

The second base model is Darcy's Law from 1856 that describes the water flow velocity in dependence upon the difference of potentials between two points in a porous medium. The flow velocity is supposed to be proportional to this potential gradient and indirectly proportional to its length. For a simplified three-dimensional system we get:

$$
\begin{aligned}
& q_{x}=-k(\Psi)_{x} \frac{\partial h}{\partial x} \\
& q_{y}=-k(\Psi)_{y} \frac{\partial h}{\partial y} \\
& q_{z}=-k(\Psi)_{z} \frac{\partial h}{\partial z}
\end{aligned}
$$

where the proportionality factor $k$ is called hydraulic conductivity $[\mathrm{m} / \mathrm{s}]$. For non-saturated systems $k$ is supposed to be an explicit function of $\Psi$. This means, the hydraulic conductivity is the same for the flow into and out of the system, i.e. the system has not hysteresis.

Besides Darcy's Law, the principle of mass conservation is often a precondition. According to this principle, any mass of water that flows into the system also has to flow out of it. Thus, any change of the mass in the system can only be due to the water flow into or from the system. A mass change in the system can only happen if the system is non-saturated, or it becomes non-saturated. The water content is symbolised by $\theta$. Furthermore, Darcy's Law assumes that the flowing fluid, i.e. water, is not compressible. This is however not realistic, and so the change of the volume of a given mass of water due to the change of the water 
pressure is explained by the thermodynamic principle of compressibility and elasticity of water:

$$
\kappa=\frac{\partial \varphi}{\varphi \partial p}
$$

where $\kappa$ is the compressibility of water, $\varphi$ the water density and $p$ the water pressure. This formula represents the isothermal compressibility, i.e. the change of the temperature caused by the change of density is ignored.

An example for a more complex model based on these principles is the SilVlow model for water movement in the soil (BLENDINGER, 1995; SCHMIDT ET AL, 1995). It simplifies Darcy's Law, taking into account only two main flow directions, vertical and horizontal, ignoring lateral flow. The model is designed to be applied to slopes and similar sites with only a two directional flow. The terrain is covered with a grid of triangular elements, each having two hydrological parameter functions, the $\mathrm{pF}$ curve $(\theta / \Psi)$ and the $\mathrm{Kr}$ curve $(\kappa / \Psi)$. For each element, the water uptake through roots is taken as sink value and added to other sinks. As input serve the precipitation and the infiltration through the upper boundary. The user defines the time steps for the simulation according to the given precipitation data. For each time step, the model calculates the system water output, potentials, water contents and flow rates according to the given boundary conditions. The equation derived from Darcy's Law for the sink values used by the model is called Seep-Flow- or Fokker-Planck-Equation:

$$
\begin{array}{r}
\partial_{t} \theta(\Psi)-\operatorname{div}(\kappa(\Psi) \cdot \operatorname{grad}(\Psi-z))=S \\
\operatorname{div}=\frac{\partial}{\partial x}+\frac{\partial}{\partial y}+\frac{\partial}{\partial z} \quad ; \quad \operatorname{grad}=\left(\begin{array}{c}
\frac{\partial}{\partial x} \\
\frac{\partial}{\partial y} \\
\frac{\partial}{\partial z}
\end{array}\right)
\end{array}
$$

The information transfer into and from the SilVlow model is actually realised through files. The input file contains the information about the infiltration rates at given points of time, the 
output file contains the potentials, water contents and flow rates for pre-selected points and time steps. Furthermore, there are thirteen different files that co-ordinate the simulation and that can be used as interface for the model. SilVlow is also designed in a way that different models for the water uptake of roots can be connected with it.

Because the model is based on Darcy's Law, it is restricted to the following conditions:

- Slopes with relatively low soil thickness or similar terrain.

- Water and soil are incompressible.

- Water flow is only due to hydraulic potential and gravitational gradients.

- There is no hysteresis.

- The water flow is predominantly described by the laminar flow in the micropores.

- The soil characteristics are homogenous inside each grid element and constant during the complete simulation time.

However, it describes well the hydrological situation for the given site conditions, and due to the portability of data from and into the model it is well suited for the interconnection with other models for more detailed simulations.

\subsubsection{Stomatal conductance}

The modelling approaches for stomatal conductance can be divided into two main categories, linear and non-linear approaches. Linear approaches are based on a linear combination of regressions with different factors such as PAR (Photosynthetically Active Radiation), global radiation $\left(\mathrm{R}_{\mathrm{g}}\right)$, water vapour saturation deficit $(\delta \mathrm{e})$, water potential $(\psi)$, air temperature $\left(\mathrm{T}_{\mathrm{a}}\right)$, leaf surface temperature $\left(\mathrm{T}_{\mathrm{S}}\right)$, concentration of $\mathrm{CO}_{2}$ in the air at the leaf surface $\left(\mathrm{C}_{\mathrm{S}}\right)$, etc., 
what makes them easy to compute and apply (JONES, 1992; BIDEL, 1994). Linear models take the general form:

$$
g_{s t o}=c_{1}+c_{2} \cdot R_{g}+c_{3} \cdot \delta e+c_{4} \cdot \Psi+c_{5} \cdot T_{S}+\ldots
$$

where $g_{\text {sto }}$ is the stomatal conductance and $c 1, c 2$, etc. are the regression constants for the respective factors. This concept is, however, considered not very exact due to the non-linear characteristic of the stomatal response to the environment, so that more complex models are generally based on non-linear approaches. SINOQUET AND LE ROUX (2000) present four major representative examples for the non-linear approaches.

The first approach is the multiplicative model presented by Jarvis (NEILSON AND JARVIS, 1975; JARVIS, 1976) that describes the stomatal conductance as the product of nonsynergistic, i.e. independent, functions:

$$
g_{\text {sto }}=g_{\text {sto } \max } \cdot f(P A R) \cdot f\left(R_{g}\right) \cdot f(\delta e) \cdot f(\Psi) \cdot f\left(T_{S}\right) \cdot f\left(C_{S}\right) \ldots
$$

where $g_{\text {sto max }}$ is the maximal stomatal conductance.

The functions are approximated by a non-linear optimisation technique to the boundary line of a given cloud of points for measured data. The main flaw of this model is that the exact degree of interaction between the observed variables is unknown. The assumption that there is no correlation between the observed variables is thus only a simple hypothesis that cannot reflect the real behaviour of stomatal conductance. However it serves to differentiate the degree of effect of each variable on stomatal conductance. Furthermore, it is difficult to optimise the values to get an acceptable regression for each variable. The approximation to the upper boundary line (frontier) or "probable upper limit of observations" (JARVIS, 1976), as shown in Figure 2-1, makes the assumption that for these values all the other variables are not limiting, so that this line reflects at best the response of the stomatal conductance to the given variable. It is possible to generate the optimal conditions for smaller plants in a laboratory, especially for crop plants, but it is almost impossible to find optimal conditions for trees or stands in the field as needed for forest research. Due to this bias, the real upper limit could be higher than observed. 


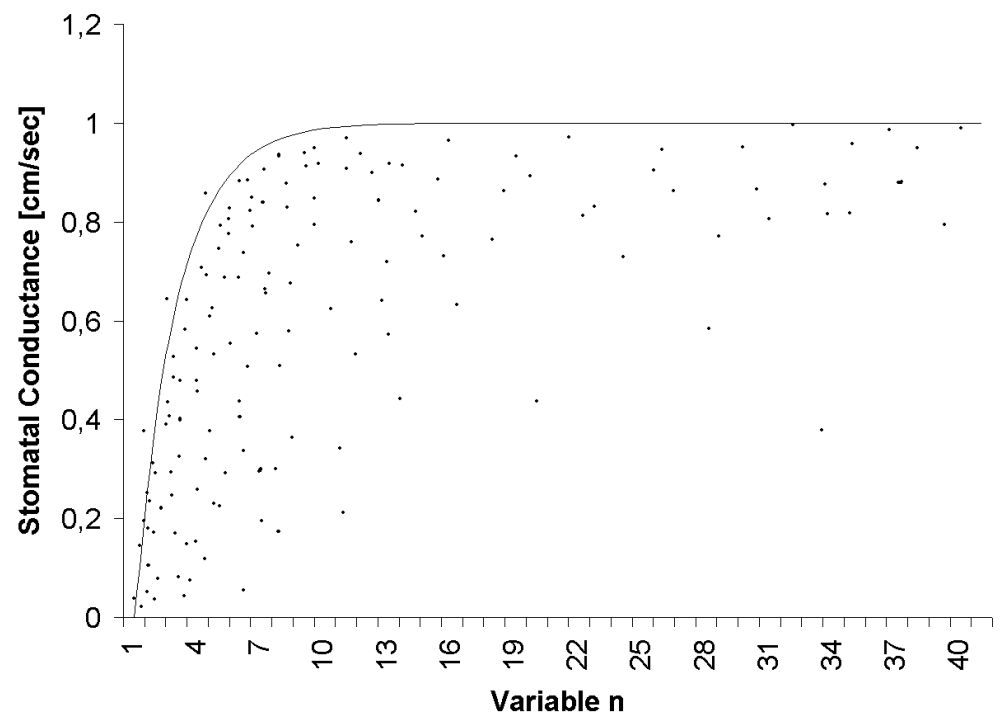

Figure 2-1: Diagram of a typical distribution of measurements of stomatal conductance $\left(g_{\text {sto }}\right)$ against an example variable $n$ as one of the variables in $g_{s t o}=g_{\max } \cdot f(P A R) \cdot f\left(R_{g}\right) \cdot f(\delta e) \cdot$ $f(\Psi) \cdot f\left(C_{S}\right) \cdot \ldots$ The line demonstrates the approximation of the upper limit of observations to get $f(n)$, where all the other variables are supposed to be not limiting the stomatal conductance. While this approximation is made for each of the variables, the curve of each function does not necessarily match the curve shape shown above.

On the other hand, the fitting can be realised using statistical analysis methods (REED ET AL., 1976; Roberts AND PitMan, 1984 cited by AdAMS ET AL., 1991). The fitting is made for each of the observed variables in the system. The number of functions used in the different models is highly variable and for each of the functions there exists a vast range of propositions (JARVIS, 1976; JONES, 1992; FARQUHAR AND WONG, 1984; CHAMBERS ET AL., 1985; LIVINGSTON AND BLACK, 1987). The model presented by Jarvis uses the following functions:

$$
\begin{gathered}
f(P A R)=\frac{a_{1} \cdot a_{2} \cdot\left(P A R-a_{3}\right)}{a_{1}+a_{2} \cdot\left(P A R-a_{3}\right)} \\
f(\delta e)=1-e^{-a_{4} \cdot \delta e} \\
f(T a)=\left(\frac{T_{S}-T_{\min }}{T_{o p t}-T_{\min }}\right) \cdot\left(\frac{T_{\max }-T_{S}}{T_{\max }-T_{o p t}}\right)\left(\frac{T_{\max }-T_{o p t}}{T_{\max }-T_{\min }}\right)
\end{gathered}
$$




$$
\begin{gathered}
f\left(\Psi_{\text {leaf }}\right)=1-e^{-a_{5} \cdot\left(\Psi_{\text {leaf }}-\Psi_{t}\right)} \\
f\left(C_{S}\right)=\left\{\begin{array}{ll}
1 & C_{S}<100 \\
1-\left(1-g_{\text {min }}\right) \cdot C_{S} / 900 & 100<C_{S}<1000 \\
g_{\text {min }} & C_{S}>1000
\end{array}\right\}
\end{gathered}
$$

where $a_{1}, a_{2}, a_{3}, a_{4}$ and $a_{5}$ are the function coefficients, $\Psi_{\text {leaf }}$ is the hydraulic potential of the leaf, $\Psi_{t}$ is the threshold value for the decrease of the stomatal conductance, and $g_{\min }$ is the minimal stomatal conductance for $C_{S}$ values greater than $1000 \mathrm{~cm}^{3} \mathrm{CO}_{2} / \mathrm{m}^{3}$ air.

YANG ET AL. (1997) applied the multiplicative model approach to simulate the stomatal conductance in Acer saccharum trees. They used PAR, leaf water potential, leaf temperature, and vapour pressure differences as variables. The resulting spline functions were fitted with least square estimation. The model HYDRO described below (DAUZAT AND RAPIDEL, 1998) uses originally the multiplicative model of Jarvis to calculate the stomatal conductance. In this model, the values were fitted for single species as for example Coffea arabica.

Second, COWAN AND FARQUHAR (1977) defined an optimisation approach based on the constant ratio $\lambda$ between water loss through evapotranspiration $(E)$ and $\mathrm{CO}_{2}$ gain through assimilation $(A)$ :

$$
\frac{\partial E}{\partial g_{s t o}} \div \frac{\partial A}{\partial g_{s t o}}=\lambda
$$

Third, BALL ET AL. (1987) presented an empirical and simpler model based on the leaf photosynthetic rate:

$$
g_{s t o}=\frac{m \cdot A \cdot h}{C_{S}+b}
$$


where $h$ is the relative air humidity, and $m$ and $b$ are function parameters. This model is considered suitable as a sub-model for canopy functioning and not as a mechanistic model by itself (Aphalo and Jarvis, cited by SINOQUET AND LE ROUX, 2000).

Finally, ZHANG AND NOBEL (1996) proposed an empirical model based on the ratio of partial pressure of $\mathrm{CO}_{2}$ in the intracellular space $\left(\mathrm{C}_{\mathrm{I}}\right)$ and the partial pressure of $\mathrm{CO}_{2}$ in the air at the leaf surface, but that does not compute the stomatal conductance directly:

$$
\frac{\mathrm{C}_{\mathrm{S}}}{\mathrm{C}_{\mathrm{I}}}=f(P A R, \delta e)
$$

Other authors use others parameter as correlation variables or include them in the multivariable approaches shown above. SchUlzE ET AL. (1985), KRAMER (1998), LIVINGSTON AND BLACK (1987) and ADAMS ET AL. (1991) present functions for the correlation of the hydraulic potential of the soil and the stomatal conductance. Alternatively, due to the influence of wind on temperature and water vapour saturation deficit and thus the indirect influence on stomatal conductance, the wind has been proposed as variable in some models (KozlowsKi ET AL., 1991; GOUdRIAAN, 1977; FARQUHAR, 1978).

\subsubsection{Transpiration}

Transpiration is a physical process that occurs at most surfaces of the plant but that is mainly concentrated on the leaves. Especially while the stomata are open for $\mathrm{CO}_{2}$ uptake (assimilation), the plant looses great amounts of water due to transpiration. The transpiration can be reduced through morphological (e.g. building a cuticula) and physiological (e.g. C4 plants) adaptation. However, all actual approaches to describe the transpiration are based on the energy balance (BIDEL, 1994; SINOQUET AND LE ROUX, 2000):

$$
R_{n}+M=H+\lambda E
$$

where $R n$ is the net radiation, $M$ the energy from metabolism, $H$ the flux of sensible heat and $\lambda E$ the flux of latent heat. The radiation balance is described below (section 2.1.4). The relatively low energy gain from metabolic processes (less than 5\%) is often neglected. 
$H$ is calculated as the heat transfer between the leaf and the air due to a heat gradient according to the thermal conductance of the boundary layer $g_{b}$ :

$$
\begin{gathered}
H=\rho \cdot c_{p} \cdot g_{b} \cdot\left(T_{s}-T_{a}\right) \\
H=\rho \cdot C_{p} \cdot g_{b} \cdot\left(T_{s}-T_{a}\right)
\end{gathered}
$$

where $\rho$ is the air density and $c_{p}$ the heat capacity of the air.

The flux of latent heat $\lambda E$ is calculated according to the conductance of water vapour from the intra-cellular space to the air $\left(g_{w}\right)$ due to the gradient between the vapour pressure in the intracellular space $\left(e_{s}\right)$, that is supposed to be saturated, and the vapour pressure of the air $\left(e_{a}\right)$ :

$$
\lambda E=\frac{\rho \cdot c_{p}}{\gamma} \cdot g_{w} \cdot\left(e_{s}-e_{a}\right)
$$

where $\gamma$ is the psychrometric constant. The conductance $g_{w}$ is a combination of the vapour conductance of the boundary layer $g_{b}$, the stomatal conductance $g_{s t o}$ and the conductance of the cuticula $g_{c}$. The interaction of $g_{s t o}$ and $g_{c}$ depends on the distribution of the stomata on the surfaces of the leaf. The water vapour deficit is influenced by the interaction of the heat of evaporation of water and the heating of the leaf due to the radiation. Because of this interplay of radiation and water vapour deficit, it is necessary to combine the equations (2-15), (2-16) and (2-17) to compute the transpiration. Penman (PENMAN, 1948) presented a first solution to this problem, the combination equation for wet surfaces, in this case a leaf:

$$
\lambda E=\frac{s \cdot \phi+\rho \cdot c_{p} \cdot g_{b} \cdot \delta_{b}}{s+\gamma\left(1+\frac{g_{b}}{g_{s t o}}\right)}
$$

with $s$, the slope of the saturation vapour curve with respect to temperature, $\phi$, the incoming radiation on the leaf surface, and $\delta_{b}$, the vapour pressure deficit of the boundary layer. Similar approaches has been proposed by SAUGIER (1974), GOUDRIAAN (1977) and JARVIS AND 
MCNAUghton (1986). TAUSEND ET AL. (2000) also used the same approach to calculate the sensitivity of transpiration to changes of the hydraulic conductivity for Coffea arabica plants.

MONTEITH (1965) tried to modify and apply the combination equation (2-18) to the whole stand, using the upper layer of the canopy as reference surface. This model was called the single leaf model, but is now often referenced as Penman-Monteith-Equation. The parameters $g_{b}$ and $g_{s t o}$ had to be modified, so that they reflected the condition of the whole stand. The conductance of the boundary layer $\left(g_{b}\right)$ has to be converted to an aerodynamic conductance for the whole air mass $\left(g_{a}\right)$ and the stomatal conductance $\left(g_{s t o}\right)$ had to be adapted to fit the whole canopy $\left(g_{s}\right)$. Equally $\delta_{b}$ is substituted by $\delta_{a}$ as the water vapour pressure deficit of the whole air mass and the net radiation $R n$ is used instead of $\phi$ :

$$
\lambda E=\frac{s \cdot R n+\rho \cdot c_{p} \cdot g_{a} \cdot \delta_{a}}{s+\gamma\left(1+\frac{g_{a}}{g_{s}}\right)}
$$

The parameters $g_{a}$ and $g_{s}$ are the critical values of the model, because they are very difficult to define well for the canopy, whereas $R n$ and $\delta a$ are relatively easy to determine. The translation from leaf scale to canopy scale has been well analysed and similar models have been proposed by other authors (FINNIGAN AND RAUPACH, 1986; JONES, 1992; MCNAUGHTON AND JARVIS, 1983).

Beside analytical methods, numerical methods can be used to solve non-linear equations like the system (2-15), (2-16) and (2-17), and thus to describe the effect of common factors on the different variables. One of the most often applied methods is the Newton-Raphson method (Li, 1966; ACTON, 1970; HAINZL, 1981; PRESS ET AL., 1992). The Newton-Raphson method is an iterative method for generating a sequence of approximations to a solution of a given equation, with the hope that it converges to the solution:

$$
x_{n}=x_{n-1}-\frac{f\left(x_{n-1}\right)}{f^{\prime}\left(x_{n-1}\right)}
$$


This method is a complement to the Taylor formula, which approximates a given function using a polynomial function. Depending on the initial conditions, the Newton-Raphson method can lead to very different results. Thus, this method is only safe if it is possible to find bounds to the roots of the function and to supply a good guess for the solution. Similar methods are the secant method and the more accurate extension of Newton's method, Newton's 2nd-order method (ACTON, 1970). More powerful are globally convergent methods as Newton's method with backtracking, Broyden's method, the hook step method and the dogleg method (PRESS ET AL., 1992).

\subsubsection{Radiation transfer and light distribution}

As described above, the light interception, and thus leaf irradiation, is one of the most important parameters used in plant modelling. It is used in most of the models for stomatal conductance (see 2.1.2), it is an integral part of the energy balance (see 2.1.3) and it is the "motor" for the photosynthesis (see 2.1.5). Thus the description of the light environment becomes an essential component of plant modelling. The structure of the plant or of the stand is regarded as an important factor that affects the light environment and the light regime of single components and thus their processes (HOST ET AL., 1990; TAKENAKA, 1994; LIST ET AL, 1994; Tsel'NiKer, 1994, 1995; PertTUnen et AL., 1996; Kurth AND ANZOlA, 1997; ANZOLA, 1998; KURTH, 1999; ESCHENBACH, 2000). In addition, the light "quality", i.e. the proportion of photosynthetically active radiation (PAR), decreases from the top to the soil level. However, some models use relatively simple approaches for the radiation transfer. The application of the Penman-Monteith equation to a canopy needs only the value of the incoming radiation at the top bounder of the canopy, and is does not need a transfer model.

The simplest models for radiation transfer are light extinction curves. These assume that the leaves are evenly distributed in the canopy space, i.e. they form a homogeneous "leaf gas", and the radiation transfer is calculated in analogy to the Beer-Lambert's Law (KURTH, 1999). This provides an exponential decrease of radiation. This approach can be extended by dividing the canopy in layers with different "leaf concentrations". This approach is not very accurate and leads to large discrepancies to measured values. In the vertical profile there are up to $10 \%$ discrepancy between simulated and measured values (LECOUSTRE, 1990) and up to $16 \%$ for the horizontal distribution (KNYAZHIKIN ET AL, 1996). 
Further extensions are the consideration of the sun position and thus the increased path for the light through the canopy, the consideration of the reflection of light from the canopy (albedo) and the consideration of the spectrometric characteristic of the leaf pigments to determine the absorption of PAR. The horizontal distribution of leaves can also be added to get more accurate results. However, at this point the structure of plants and/or stands plays an important role for describing the three-dimensional components of light distribution and radiation transfer (KuUluvainen And PukKala, 1987; Anzola, 1998; De Castro and Fetcher, 1998; DAUDET ET AL, 1999; KURTH, 1999), and thus, it can not be satisfied by models of this category.

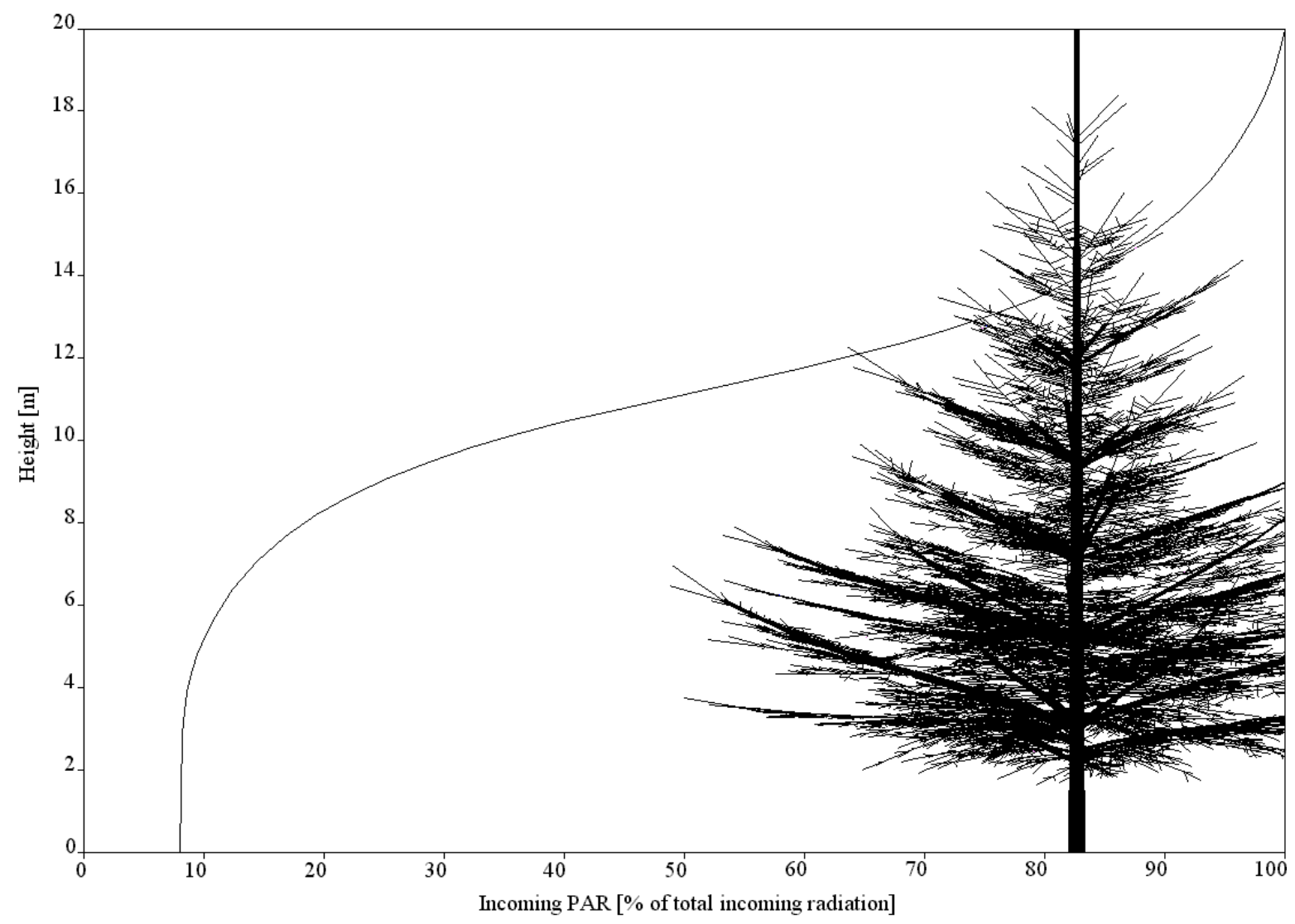

Figure 2-2: Typical light extinction curve according to Beer-Lambert's Law taking into account the leaf-mass density at different heights.

\subsubsection{The models MIR and MuSc}

The models MIR (Mapping Intercepted Radiation) and MuSc (Multi-Scattering) were developed at the CIRAD Plant Modelling Unit in France by Jean Dauzat and form part of a project for modelling hydraulic transfer (DAUZAT AND HAUTECOEUR, 1991; DAUZAT, 1994; 
DAUZAT AND EROY, 1997; RAPIDEL, 1995; RAPIDEL ET AL. 1999). The model MIR is based on the Turtle principle developed by DEN DULK (1989). The sky is represented by a hemisphere that is divided into 46 sectors (Figure 2-3). For each direction corresponding to the sectors the quantity of incoming PAR is calculated. The turtle is placed above the plant or stand. These are represented by simplified scene files (“.scn”) generated from AMAP scene files (“.sce”). In addition, a ".scu" file containing information for the needed plot inside the scene is required. This file contains the limits of the plot and its geographical co-ordinates (latitude and longitude) needed to calculate the position of the sun. In the used co-ordinate system $\mathrm{X}$ corresponds to the East, $\mathrm{Y}$ to the North, and $\mathrm{Z}$ to the altitude. For a given moment the model calculates the amount of intercepted radiation for each visible pixel of the scene and calculates the brightness according to the pixel area. Direct and diffuse components of the global radiation are calculated in relation to the extra-terrestrial radiation using formulas proposed by Jong (1980, cited by SPITTERS ET AL., 1986). To achieve this, two formulas are applied. First, the Standard OverCast sky distribution (SOC) of MOON AND SPENCER (1942) for completely clouded sky. And second, the formula of ANDERSON (1966) for clear sky. According to the difference between global and extra-terrestrial radiation, a percentage of cloud cover is calculated and the respective formula applied. Thus, the brightness is linearly proportional to the cloud cover.

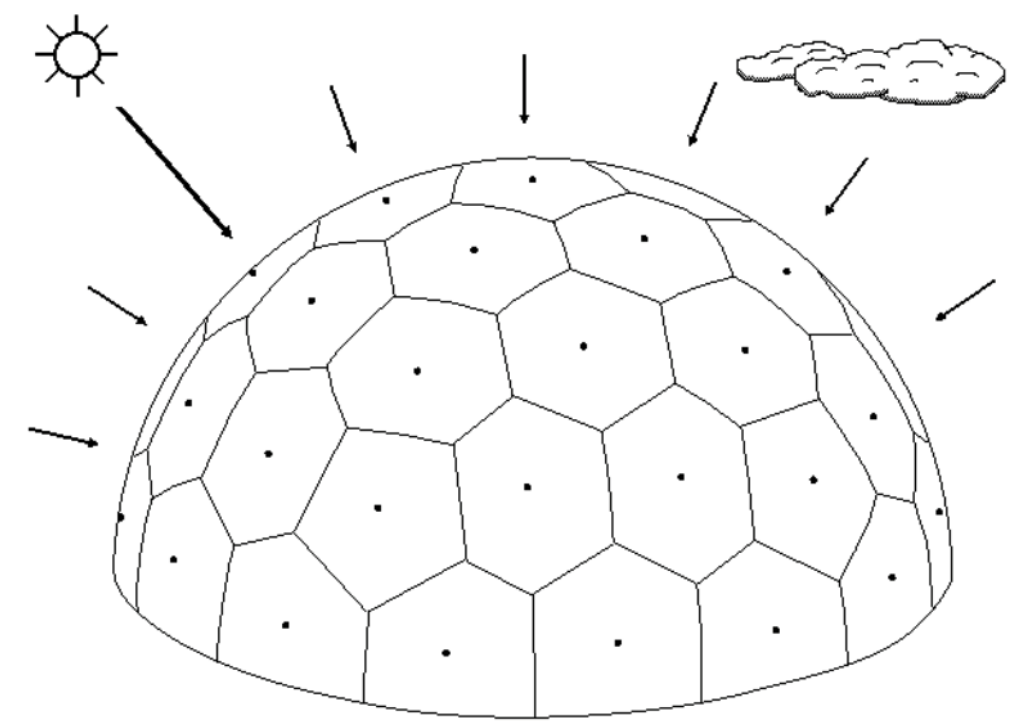

Figure 2-3: Turtle model for the sky hemisphere. The turtle is divided in 46 sectors. For each sector the incoming radiation is calculated. Cloud cover is calculated from the difference between global and extra-terrestrial radiation. 
MIR generates a map of the light interception with different options for each sector. The model is currently being modified in a way that the number of sector can be varied according to needs of specific projects (DAUZAT AND NOUGUIER, personal communication).

The model MuSc calculates the scattering of the intercepted light due to reflection and transmission, at the different horizontal layers of the vegetation and the soil. Because reflected and transmitted radiation can also be absorbed, reflected or transmitted, the model has to take into account the multiple scattering. In contrast to MIR, MuSc calculates the radiation for each layer and not for each element. For each layer, MuSc uses the information from MIR to calculate the total intercepted radiation in that layer. The radiation transfer in each layer is calculated with the Beer-Lambert's Law. The different events are calculated in a numerical process and the procedure is repeated until the radiation fluxes are negligible. The soil is considered as special layer without transfer rate and with its own reflection coefficient. The intercepted radiation for each layer is distributed among the leaves in proportion to their area. Both programs are part of the ARCHIMED model shell, which furthermore contains the programs Biomscene and Radbal. ARCHIMED is a series of concatenated programs destined to calculate the radiation and thermodynamic balance of a scene.

\subsubsection{Photosynthesis}

Photosynthesis is one of the most important processes in plant physiology. There exists a vast range of models that simulate both directly and indirectly, this process. Photosynthesis depends on many variables, the most important being the light intensity, but also light quality, temperature, $\mathrm{CO}_{2}$ concentration in the air, the water vapour deficit, the physiological status of the leaf, and the concentration of air pollutants. The simulation of photosynthesis is especially important for C-allocation models as source of assimilates, e.g. ECOPHYS (HOST ET AL., 1990), MADEIRA (LIST AND KÜPPERS, 1998), SIMWAL (BALANDIER ET AL., 2000), EMILION (BOSC, 2000) and ALMIS (ESCHENBACH, 2000).

Photosynthesis can be defined simply as the uptake of $\mathrm{CO}_{2}$ and $\mathrm{H}_{2} \mathrm{O}$ for the production of glucose and the emission of oxygen using the energy from the light, as described by the equation: 


$$
\begin{array}{r}
6 \mathrm{CO}_{2}+6 \mathrm{H}_{2} \mathrm{O} \stackrel{\Delta G}{\longrightarrow} \mathrm{C}_{6} \mathrm{H}_{12} \mathrm{O}_{6}+6 \mathrm{O}_{2} \\
\Delta G=+2875 \mathrm{~kJ} \text { from light }
\end{array}
$$

BLACKMAN described in 1905 for the first time the effects of light and temperature on photosynthesis (BAYRHUBER ET AL., 1991). He discovered that under constant temperature, the photosynthetic rate increases with increasing light intensity, until it reaches a point at which photosynthesis is maximal. He called this point the light saturation point. Beyond this point the photosynthetic rate stays constant. For the temperature he discovered that there exist a temperature minimum point, a temperature optimum point with maximal photosynthetic rate, and a temperature maximum point. Furthermore, he found that the effect of temperature is low for low light intensity, and high for stronger light intensity. The $\mathrm{CO}_{2}$ concentration of the air has a similar effect on photosynthesis as the light intensity, with a $\mathrm{CO}_{2}$ saturation point for given temperature and light intensity.

Figure 2-4 shows the light saturation curve, the $\mathrm{CO}_{2}$ saturation curve and the temperature curve of photosynthesis. Photosynthesis is divided into two reaction chains. The first chain contains the light or primary reactions, which depends on the light intensity, but are relatively temperature independent. The second chain of reactions is temperature dependent but doesn't need light; these are the darkness or secondary reactions. The primary reactions deliver the products needed for the secondary reactions. By low light intensity only a small amount of substances is produced in the primary reactions. These products are rapidly consumed in the secondary reactions, even by low temperatures. Thus, an increase of the temperature does not strongly affect the photosynthetic rate. By high light intensity, the demand of the secondary reaction can be satisfied by the primary reactions, so that the photosynthetic rate increases with higher temperatures, until the enzymatic activity ends due to enzyme deterioration. The shape of the different curves, the saturation points and the maximum, minimum and optimum points are different for each plant and even for different types of leaves within the same plant.

The effect of water is directly correlated to the stomatal conductance. Drought stress conditions induce a reduction of the stomatal conductivity because the stomata get closed (see 2.1.2), the $\mathrm{CO}_{2}$ uptake is thus also reduced and so also the photosynthesis. 
a)

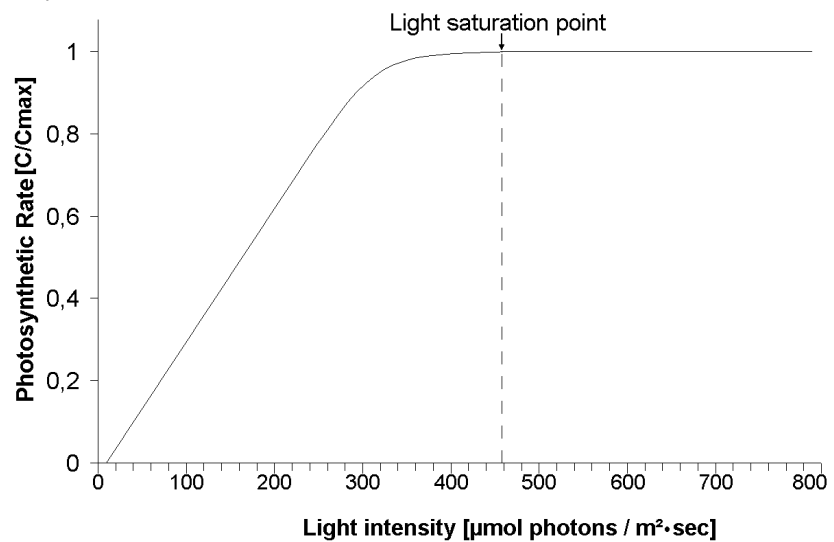

c) b)

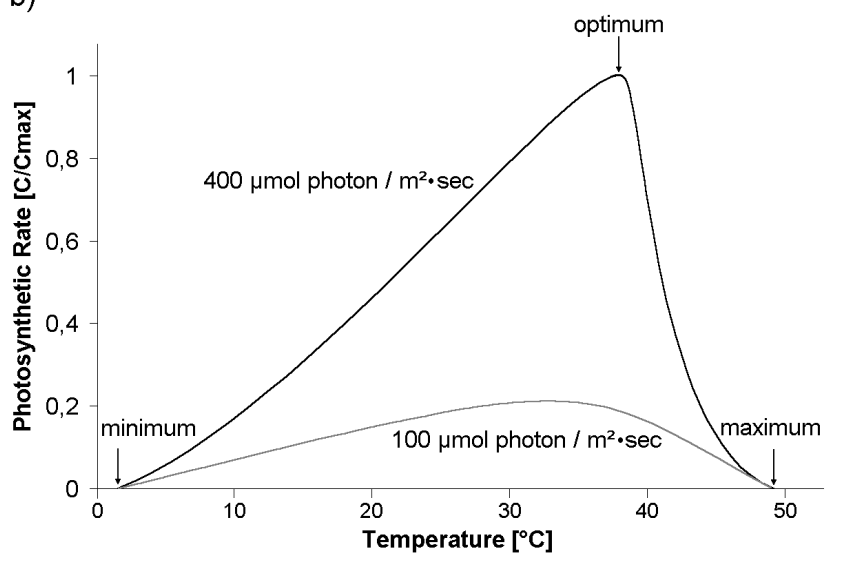

$400 \mu \mathrm{mol}$ photon $/ \mathrm{m}^{2} \cdot \mathrm{sec}$

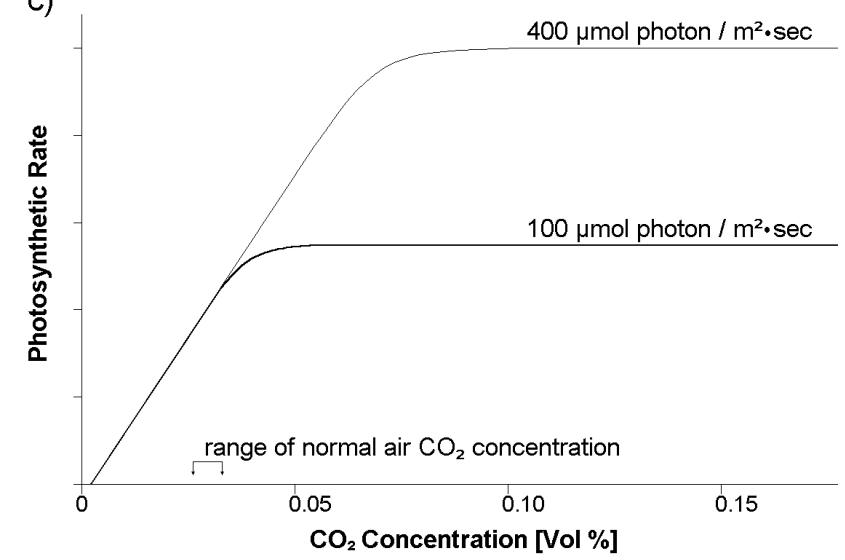

Figure 2-4: Photosynthetic rate in dependence on light intensity (a), temperature (b) and air $\mathrm{CO}_{2}$ concentration $(\mathrm{c})$. The effects of temperature and $\mathrm{CO}_{2}$ concentration are greater by increasing light intensity. Temperature regulates enzyme reactions, and a higher $\mathrm{CO}_{2}$ concentration means a higher input. However, the whole process is principally regulated by the photochemical reactions, and thus by light intensity.

These four processes build the base of most photosynthesis models. However, we can classify the models into three main approaches (SINOQUET AND LE ROUX, 2000). The first approach includes models that compute the assimilation indirectly. The production of assimilates $(\mathrm{P})$ is assumed to be proportional to some factor, normally leaf area $\left(\mathrm{A}_{1}\right)$, leaf mass $\left(\mathrm{W}_{1}\right)$, light absorption or transpiration:

$$
\begin{gathered}
P=\sigma_{l} W_{l} \\
P=\sigma_{l} A_{l} \\
P=L U E \cdot P A R
\end{gathered}
$$




$$
P=W U E \cdot E
$$

with $\sigma_{1}$, the leaf specific activity $\left(\mathrm{g} \mathrm{C} \mathrm{g}^{-1}\right.$ in $2-22 \mathrm{a}$ or $\mathrm{g} \mathrm{C} \mathrm{m}^{-2}$ in 2-22b), LUE, the light use efficiency $\left(\mathrm{g} \mathrm{C} \mathrm{MJ}^{-1}\right)$, WUE, the water use efficiency $\left(\mathrm{g} \mathrm{C} \mathrm{kg} \mathrm{H}_{2} \mathrm{O}^{-1}\right)$.

The model MADEIRA (LIST AND KÜPPERS, 1998) presents a photosynthesis sub-model which assumes that the light saturation point is not reached or only for short, negligible periods. By combining the normal light saturation curve with measurements over long periods of time and on the whole tree, the model provides a linear seasonal function for the carbon gain. The model calculates the assimilation in a season from leaf area and the light climate. A similar approach is followed in numerous forest growth models operating at a time scale of seasons or growth periods (years) (MÄKELÄ, 1986; PERTTUNEN ET AL., 1996).

The second type of approach includes models that calculate directly the assimilation through a multiplicative, empirical function similar to (2-6):

$$
A=A_{\max } \cdot f(P A R) \cdot g_{1}\left(T_{a}\right) \cdot g_{2}\left(C_{a}\right) \cdot g_{3}(\delta e) \cdot g_{4}(\Psi) \cdot g_{5}(N)
$$

where $A_{\max }$ is the maximal assimilation, $f(P A R)$ is an empirical function for the effect of PAR on photosynthesis, and $g_{n}$ are the multiplicative functions for the other possible variables. GOUDRIAAN (1982) proposed a rectangular hyperbolic model for $f(P A R)$ that is used in other important models such as ECOPHYS (HOST ET AL.,1990):

$$
C E R=\left(\left(F_{\max }+R_{d}\right) \cdot P_{e} \cdot P P F D /\left(P_{e} \cdot P P F D+F_{\max }+R_{d}\right)\right)-R_{d}
$$

where $C E R$ is the carbon exchange rate, $F_{\max }$ the maximal photosynthetic rate, $R_{d}$ the dark respiration rate, $P_{e}$ the photosynthetic efficiency at low energy level, PPFD the photosynthetic active photon flux density. However it ignores the $\mathrm{CO}_{2}$ concentration. The model ALMIS (ESCHENBACH, 2000) calculates photosynthesis in dependence on stomatal conductance, PPFD and temperature. 
The third approach describes the biochemical models in conjunction with a stomatal conductance model, presented by Farquhar (FARQUHAR ET AL., 1980):

$$
A=\min \left(W_{c}, W_{j}\right)+R_{d}
$$

where $W_{c}$ is the carboxylation rate in dependence of the Rubisco activity, $W_{j}$ is the carboxylation rate in dependence of the regeneration of Rubisco, and $R_{d}$ is the rate of $\mathrm{CO}_{2}$ evolution in light that results from processes other than photorespiration. This model is used in a modified form by the models EMILION (BOSC, 2000) and SIMWAL (BALANDIER ET AL., 2000). LE ROUX ET AL. (1999) adapted the models of Jarvis and of Farquhar and parameterised them for walnut (Juglans regia).

\subsubsection{Wind profile}

The structure of trees has great effects on wind characteristics. In the canopy the wind is characterised by turbulent movement. The atmospheric turbulence and the analogously defined turbulent diffusion are the main "motors" for the exchanges between the canopy and the atmosphere and the deposition of pollutants and nutrients (CONSTANTIN, 1993; IBROM, 1993; NÜTZMANN, 1999). They are an important part of SVAT models (Soil Vegetation Atmosphere Transfer Models).

Most of these models are based on wind velocity. Due to the complexity of the canopy, especially the density and inhomogeneity, it is very difficult to provide an exact model for the distribution of wind velocity and turbulence within the stand. Many authors assume a homogeneous canopy for the calculation of wind velocities. The simplest model for the wind velocity by lacking a wind profile is given by the formula:

$$
v(h)=v_{0}\left(\frac{h}{h_{0}}\right)^{c}
$$

where $v(h)$ is the wind velocity at the height $h, v_{0}$ the wind velocity at the reference height $h_{0}$, and the factor $c \approx 0,3$ for forests (HÄCKEL, 1999). 
The classical model for the vertical wind profile is given by the combination of a logarithmic function above the canopy (2-27), and an exponential function within the canopy (2-28), for a closed and homogeneous canopy (THOM, 1975; CHOUdHuRY AND MONTEITH, 1988; LHOMME ET AL., 1994):

$$
\begin{gathered}
v(h)=\frac{U *}{k} \cdot \ln \left(\frac{h-d}{z_{0}}\right) \\
v(h)=v_{0} \cdot \frac{\ln \left(\frac{h_{c}-d}{z_{0}}\right)}{\ln \left(\frac{h_{0}-d}{z_{0}}\right)} \cdot e^{-n \cdot\left(1-\frac{h}{h_{c}}\right)}
\end{gathered}
$$

where $k$ is the von Karmán constant $(k=0.4), U^{*}$ the shear stress velocity, $h_{c}$ the upper height of the canopy, $d$ the displacement height for the logarithmic function $\left(d=0.13 \cdot h_{c}\right), z_{0}$ the roughness length $\left(z_{0}=0.63 \cdot h_{c}\right)$, i.e. the point at which the velocity calculated by the logarithmic law would be zero, and $n$ a correction coefficient for the leaf distribution $(n=$ $2.5)$. Figure $2-5$ shows the typical wind profile as calculated by the functions (2-27) and (228) outside (a) and inside (b) of a $20 \mathrm{~m}$ high stand, with a wind velocity of $30 \mathrm{~m} / \mathrm{s}$ measured at a height of $30 \mathrm{~m}$. Inside the stand, both $d$ and $z_{0}$ are higher than outside because they are calculated in relation to the stand height (BIDEL, 1994). $U^{*}$ is calculated using the reference values $h_{0}$ and $v_{0}$ in (2-27). For the logarithmic curve outside the stand (a), $d$ is set to 0 .

NÜTZMANN (1999) describes a more complex model for the turbulent transfer in the canopy space based on the Transilient Turbulent Theory (TTT). The main drawback of most models for forest atmosphere is that they are often limited to the canopy space or short above it, so that the effects of the atmospheric boundary layer are not taken into account. Thus, it is very difficult to connect complex tree structure models with atmospheric transfer models. BRUNET (1997) asserts that this connection is often inefficient, due to the difference of information content.

Actual models use more general structure parameters to establish a relation to the momentum transfer such as the one-sided LAI and the projected area of the other plant elements 
(branches, fruits, etc.). The relative density of leaves and other organs can be calculated through the relation of these projection indices with the height of given layers in the canopy. These values help to calculate the aerodynamic resistance of a stand, using as reference measures in the wind tunnel (BRUNET ET AL., 1994; BRUNET ET AL. 1996; NÜTZMANN, 1999). However, in most of these models the wind components are simplified and only onedimensional components are used.

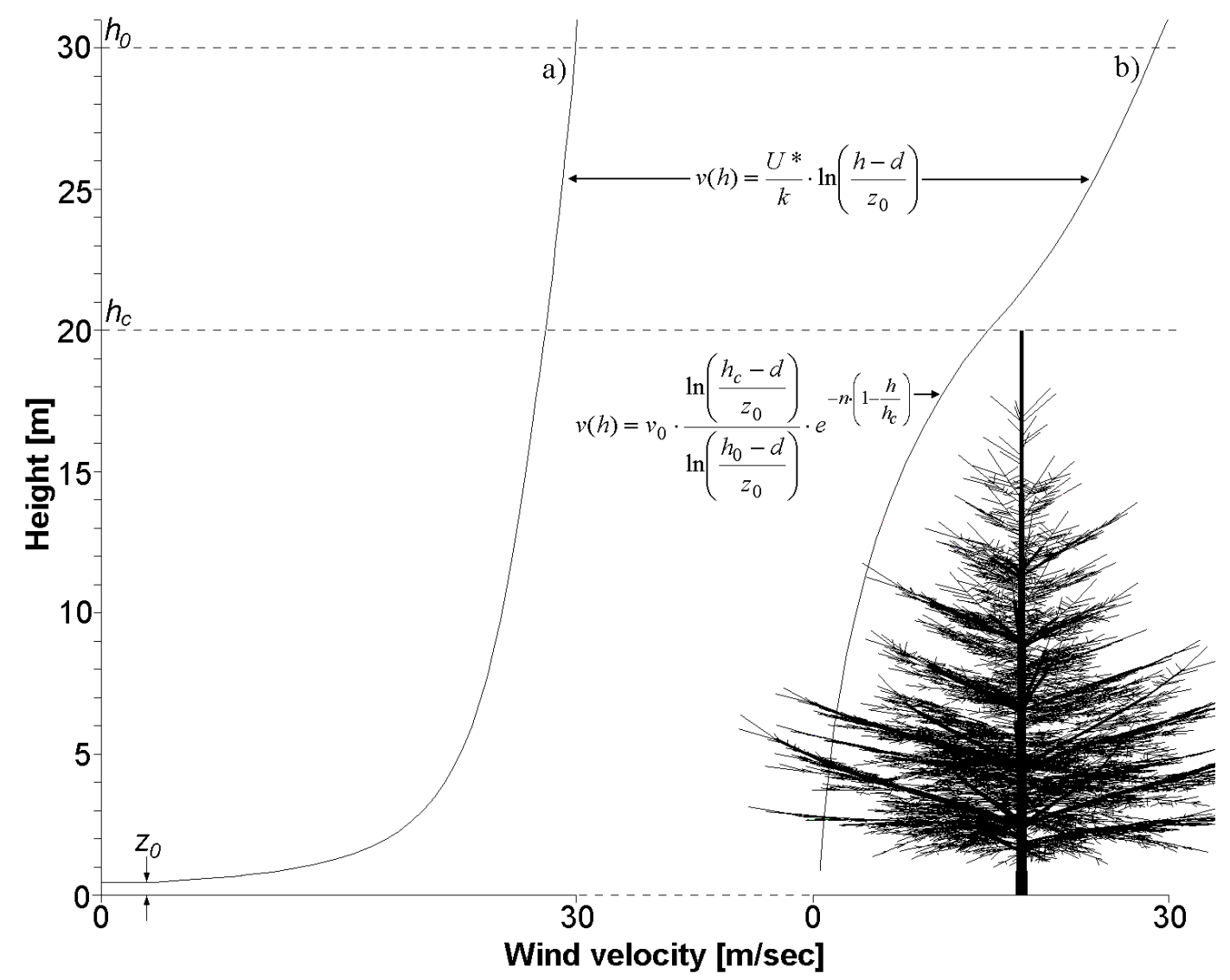

Figure 2-5: Characteristic wind profiles outside (a) and inside (b) a tree stand. For open fields and for the space above the canopy, the wind profile follows a logarithmic law. Inside the canopy, the wind velocity profile follows an exponential law. These formulas are valid under the assumption of a homogeneous canopy. For (a) is $z_{0}=0.13 \mathrm{~m}$ and $d=0 \mathrm{~m}$, and for (b) $z_{0}=2.6 \mathrm{~m}$ and $d=12.6 \mathrm{~m} . v_{0}=30 \mathrm{~m} / \mathrm{s}, h_{0}=30 \mathrm{~m}, \mathrm{hc}=20 \mathrm{~m}$ and the von Karmán constant $k=0.4$.

\subsubsection{Water transfer in plants}

Water transfer through the plant is important for the transpiration, as already mentioned in chapter 2.1.3. Mechanistic models consider the plant as a series of resistances between the soil and the atmosphere (VAN DEN HONERT, 1948; ROBERTS, 1976; TYREE, 1993; YANG AND TYREE, 1993). The simplest models for the water transfer consider the plant or stand as only 
one compartment, like the single leaf approach described by MONTEITH (1965). The next step in the development of water transfer models was subdividing the plant into many different compartments. JARVIS ET AL. (1981) and AMÉGLIO (1991) presented simple models with only two compartments which permit to calculate the water flux through the plant. CRUIZIAT ET AL. (1989) developed a model with four compartments for conifers. They differentiated stem, branches in the lower part of the crown, branches in the upper part of the crown and foliage. These compartments showed different potential gradients. Similar approaches included the roots as a new compartment. RUNNING ET AL. (1975) developed a model with two compartments that concentrated on the role of roots and the water transfer from the roots to the sapwood. This model calculates the water flow in response to the demand of the plant to maintain the hydraulic balance under the aspect of mass conservation. ROBERTS (1976) and EDWARDS ET AL. (1986) also developed models that included the roots as compartment. The model by Edwards et al. calculates the water flow according to Darcy's Law (see 2.1.1), but the plants are divided into only four compartments. Thus, the water flow is determined principally through the length of the stem compartment, and the total branch length is reduced to a single compartment.

SHINOZAKI ET AL. (1964) defined the vertical distribution of non-photosynthetic organs. Their model bases on the assumption that the amount of leaves above a given height $z$ must be supported by an amount of non-photosynthetic organs $C$ (branches and stem). Thus both amounts are proportional. Figure 2-6-C shows the vertical distribution of leaves $(\Gamma(z))$ and non-photosynthetic organs $(C(z))$. They conclude that a set of leaves is supported by a basic unit pipe whose thickness is constant (Figure 2-6-A). A plant stand is a set of these units and builds the unit pipe system (Figure 2-6-B). A plant is in turn also a unit pipe system (Figure 2-6-D). This scheme can be repeated for single branch systems. This is the so called pipe model theory.

This theory was proved to be true for most herbaceous communities. With some extensions, Shinozaki et al. also managed to explain the irregularities found at the bottom of trees and tree communities. The leafless part of the stem causes a bending of the correlation curve between accumulated leaf mass and branch mass. The die-back of the lower leaves and branches results in an accumulation of useless pipes at the bottom of the tree which do not longer 
support any living leaves. This is shown in figures Figure 2-6 and Figure 2-7. This new formulation of the pipe theory is called the pipe model theory of tree form.

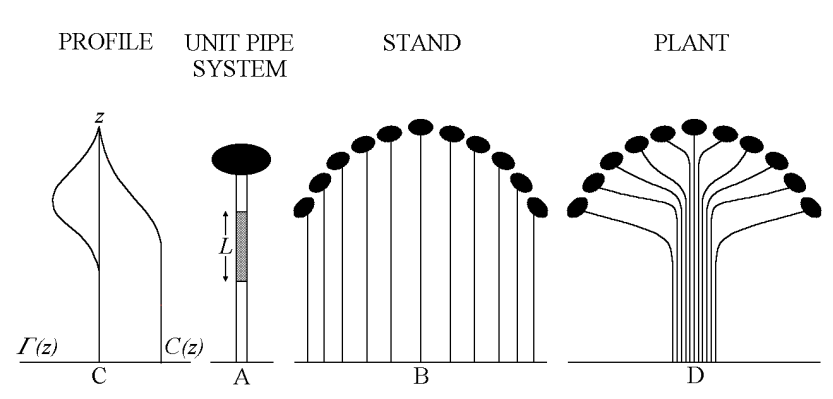

Figure 2-6: Diagrammatic representation of the simple pipe model of plant form (after SHINOZAKI ET AL., 1964). Diagram C shows the vertical distribution of leaves $(\Gamma(z))$ and branches $(C(z))$. A is the unit pipe, that can be united into a unit pipe system as the stand (B) or a single plant (D)

Figure 2-7: Diagrammatic representation of the pipe model of tree form, showing the successive accumulation of disused pipes in the trunk associated with the progress of tree growth (after SHINOZAKI ET AL., 1964)

This constancy of the cross-sectional area of sapwood in proportion to the supported leaf mass is an important relationship for both the hydraulic and mechanic architecture of plants. The development of this model was an important step toward more accurate structural-functional models (ChiBA ET AL., 1994; ANZOLA, 1998; KURTH, 1999; ChIBA, 2000).

A more accurate structure model as base for the hydraulic architecture was developed by TYREE (1988). He divided a 10 meters high Thuja occidentalis tree into 4107 segments using the nodes as delimitation. He conserved the topological information of each segment in form of an index which denominates each segment and its predecessor. For each segment he also stored length, diameter and leaf area. This information was contained in a hydraulic map. The roots form a single compartment of their own, with one hydraulic resistance and capacity. These values are re-calculated according to the demand of the simulation. For the parameterisation of the hydraulic characteristics, Tyree centred the spatial discretisation on the middle of the segments, and not on the nodes. He calculated the water flow according to Darcy's Law, assuming homogeneity in the cross-sections of the segments and taking the principle of mass conservation into account. The resulting differential equations can be combined into: 


$$
F_{j, n}^{\text {in }}-F_{j, n}^{\text {out }}=\left[c_{j, n} \cdot D_{t}^{+} \cdot \Psi_{j, n}+E(t) \cdot l_{j}\right] \cdot \Delta \kappa_{j} A_{j}
$$

where $F_{j, n}^{\text {in }}$ is the water flow into segment $j$ at time $n\left[\mathrm{~kg} \cdot \mathrm{s}^{-1}\right], F_{j, n}^{\text {out }}$ the water flow out of segment $j$ at time $n\left[\mathrm{~kg} \cdot \mathrm{s}^{-1}\right], c_{j, n}$ the capacity $\left[\mathrm{kg} \cdot \mathrm{dm}^{-3} \cdot \mathrm{Mpa}^{-1}\right], D_{t}^{+}$the operator for the temporal forward difference, $\Delta \kappa_{j} A_{j}$ the approximation for the volume of segment $j\left[\mathrm{dm}^{3}\right], l_{j}$ the leaf area per volume of segment $j\left[\mathrm{~m}^{-1}\right]$.

TAUSEND ET AL. (2000) investigated how different factors affect the transpiration, and thus the water flow in coffee (Coffea arabica) cultivars. They measured transpiration per unit leaf area, leaf water status, stomatal conductance leaf boundary layer conductance, crown conductance and total hydraulic conductance of the system between the soil and the leaves to investigate the reactions of water flow to partial defoliation.

A further process that influences the water flow is the xylem embolism or cavitation, the closure of the xylem vessels through air inclusion (CRUIZIAT ET AL., 2001). The accumulation of cavitation incidents leads to a reduction of the conductivity (SPERRY ET AL., 1988). This conductivity loss is possibly reversible (FRÜH, 1995). It is possible to simulate it using a vulnerability curve, i.e. a function which represents the conductivity loss in dependence upon the hydraulic potential or water content (EDWARDS AND JARVIS, 1982; EDWARDS ET AL., 1986).

\subsubsection{The model HYDRA}

FRÜH (1995) developed a numerical model for the hydraulic system of trees. This model is restricted to the above-ground part of the tree. However, an interface to the model SilVlow (see section 2.1.1) has been developed to complete the system. The model takes into account the network characteristics of tree structures to calculate water flow. The structure is first divided into non-branched segments of constant cross-section (axis segments) and then evaluated with a discrete method. Similar to the model of TYREE (1988), HYDRA is based on the one-dimensional formulation of Darcy's Law and on the principle of mass conservation. Tyree calculated the conductivity at the middle of the segments. Früh recommends a 
discretisation method whose grid nodes coincide with the branching nodes to avoid the necessity of weighting the conductivity values before and after the node (Figure 2-8 and Figure 2-9) and to ensure numerical consistency.

The model assumes that within an axis segment the capacitance and the axial conductivity are homogeneous. These three basic assumptions lead to the following non-linear diffusion equation:

$$
\frac{\partial}{\partial x}\left[K(x, P) \frac{\partial \Psi}{\partial x}\right]=c(x, P) \frac{\partial \Psi}{\partial t}+E(t) l(x)
$$

where $K$ is the hydraulic conductance, $E(t)$ the transpiration, $l(x)$ the local leaf area index, $P$ the hydrostatic pressure, and $c$ the hydraulic capacitance. For conifers, the model assumes a uniform distribution of leaf area over the surface of the segment. Leaf area serves directly as transpirational surface for the segment, and the leaf capacities are added to the segment capacities. For deciduous trees each leaf is represented by two segments, one for the petiole, and one for the blade (SCHULTE, personal communication), thus allowing a more realistic dynamic of the hydraulic system.
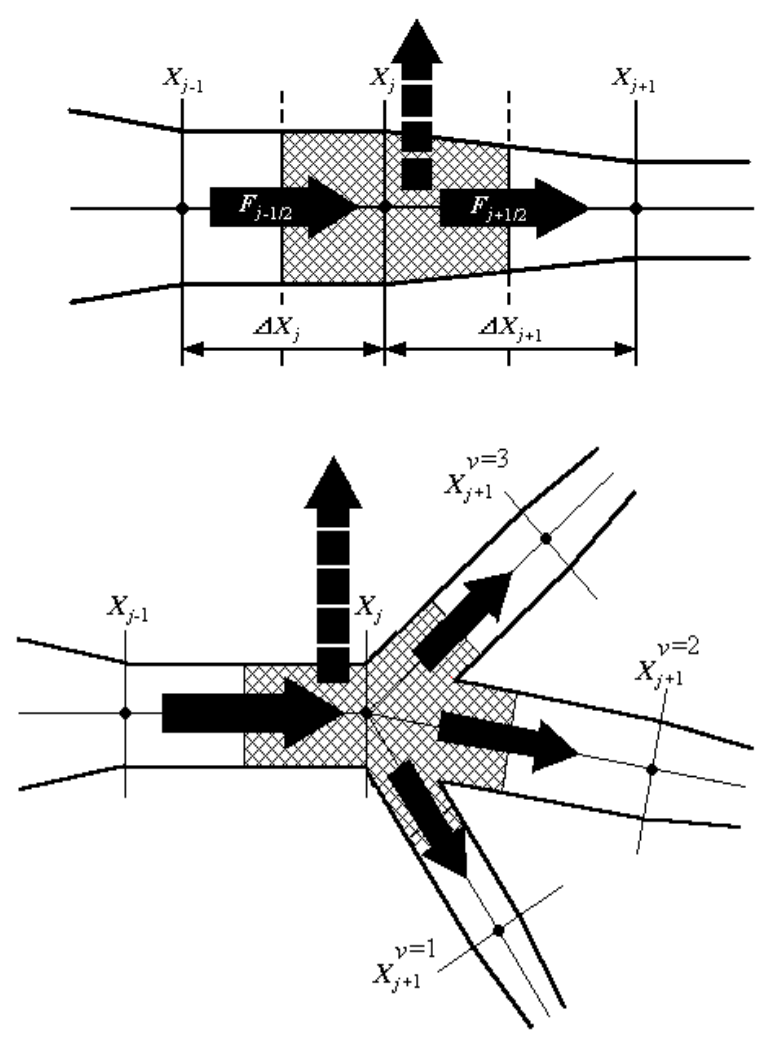

Figure 2-8: Spatial discretisation for an unbranched part of the hydraulic network with variable cross sections. For the shaded region, mass conservation is assumed. The arrows indicate water flow and transpiration (after FRÜH, 1995).

Figure 2-9: Spatial discretisation scheme in the vicinity of a branching node. For the shaded cell, mass conservation is assumed. The arrows indicate water flows across the border and transpiration (after FRÜH, 1995). 
Due to the selected boundary conditions, an initial boundary value problem (IBVP) results. To solve this non-linear IBVP the finite difference method of Douglas and Jones is applied. This is based on a Crank-Nicholson scheme with a predictor-corrector method. Früh extended the system to apply it to hydraulic systems with variable cross sections as shown in Figure 2-8, and with branching points as shown in Figure 2-9, through discrete approximation of the adjacent cell volume. The parameterisation of the IBVP follows a principle of Tyree (TYREE AND SPERRY, 1988), deriving the local initial hydraulic conductivities and capacities according to the local geometrical characteristics. Initial conductivity depends on the xylem structure and is assumed to be free of any embolism. Initial capacity depends on axial volume and leaf areas. For the spatial discretisation, segment lengths are selected following two numerical criteria:

a) Uniform accuracy: Spatial steps are large where moderate gradients exist, and small where gradients are steep.

b) Locally limited variation of step-widths: A very small step cannot follow a large step. This is necessary to prevent the reduction of convergence order by the transition from constant to variable spacing.

There must be a compromise between both criteria because gradients may vary extremely, and no information is yet available about the spatial pattern of gradients. The discretisation is also limited by the natural branching of plants. The branching structure containing only branching points and terminal nodes forms the base grid for a multi-step discretisation algorithm, which generates a hydraulic map similar to that from Tyree. Both topological and geometrical data for each element is contained in this map file. For the temporal discretisation an algorithm from HoRNUNG AND MESSING (1984) was used. Time steps are adjusted in a way that the change of water content $\theta$ occurs accordingly to a given optimum $\Delta \theta_{\text {opt }}$.

The program was initially strongly coupled with GROGRA (KURTH, 1994), which provides the base grid files from simulated or mapped trees. A separated module called DISC generates the hydraulic map. However, to also get access to data generated with the AMAP software, Lanwert (LANWERT, 1997; LANWERT ET AL, 1998) developed a first tool that generates base grid maps from the architecture files from AMAP. 
The model was initially tested on a Thuja occidentalis tree previously mapped by TYREE AND Sperry (1988), a 14 years old Picea abies (L.) tree from Solling, Germany, and artificial Picea abies (L.) trees generated with GROGRA. Further tests were made with mapped Picea abies trees from Solling with accurate data about needle mass and area (ANZOLA, 1998; FRÜH AND KURTH, 1999). This model is a powerful and numerically stable calculation tool and allows theoretically well-founded hypotheses about the behaviour of the hydraulic system (FRÜH AND KURTH, 1999).
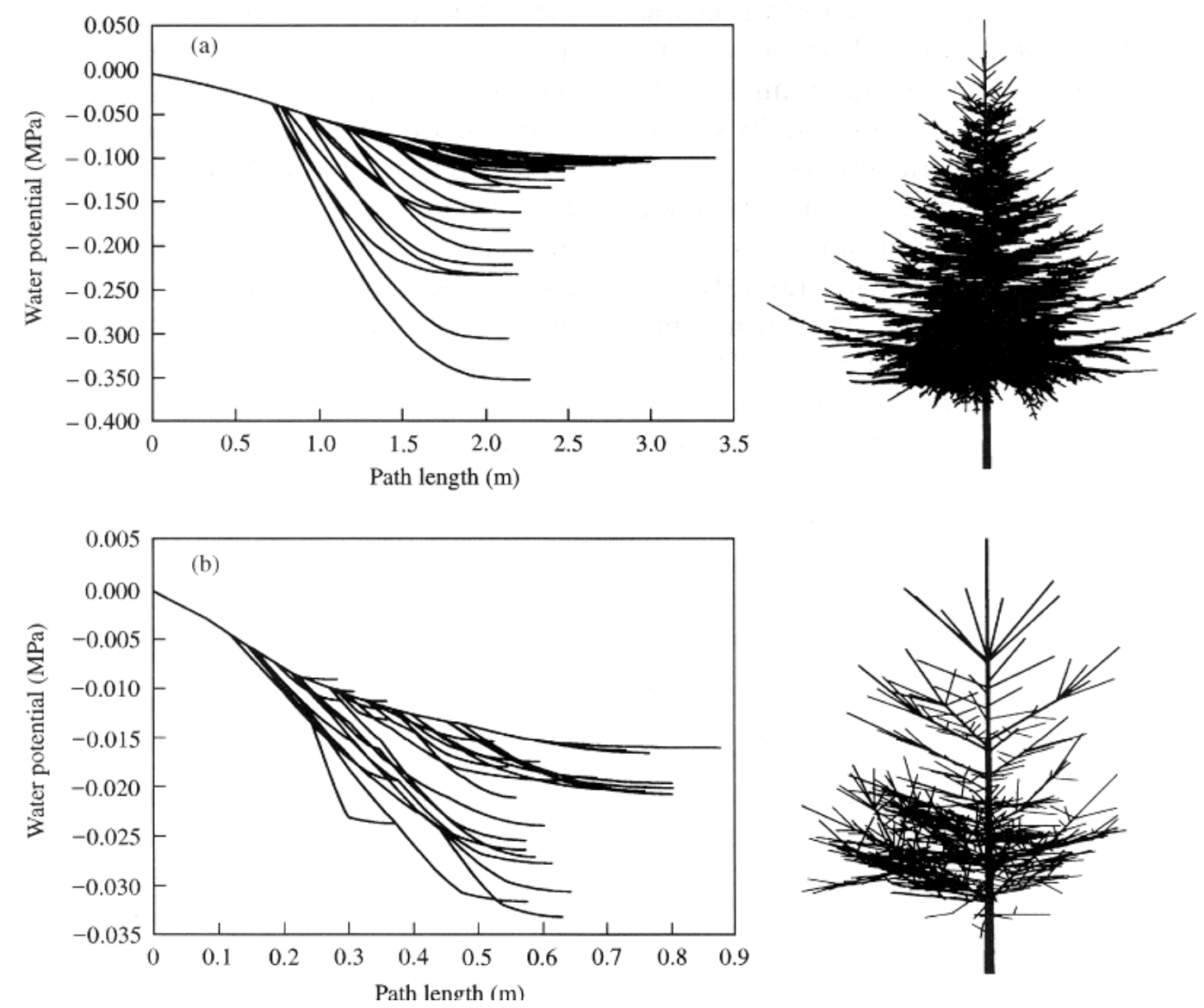

Figure 2-10: Steady state water potential profiles generated by HYDRA. After selecting any number of nodes from the hydraulic map, HYDRA calculates the water potentials along the resulting flow paths. (a) shows the profile for a spruce tree simulated with GROGRA. (b) shows the profile for a young spruce tree from Solling mapped by ANZOLA (1998). After FRÜH AND KURTH (1999).

\subsubsection{The model HYDRO}

DAUZAT (1993) also developed a model for the simulation of the hydraulic system of trees called HYDRO. HYDRO is a complex model that integrates different sub-models dedicated to water flow in plants. The model uses data generated by MIR/MuSc (Section 2.1.4.1) to 
calculate the radiation balance and the transpiration rate of the plant or stand according to the formulas $2-15,2-16$ and 2-17 in section 2.1.3. The stomatal conductance is calculated in analogy to the multiplicative models of Jarvis (formula 2-6), FARQUHAR (1978) and FEDERER (1980). For the wind velocity Dauzat used the formulas 2-27 and 2-28.

Water flow in a branch is a function of the transpiration through the sustained leaf mass. The model is thus based on the principle of water potential differences. The conductivity of the xylem is reflected by the resistance that it opposes to water flow and the water content changes:

$$
\begin{gathered}
\Delta \Psi=-\mathrm{R} \cdot \Phi \\
\Delta \mathrm{V}=-\mathrm{C} \cdot \Delta \Psi
\end{gathered}
$$

where $\Delta \Psi$ is the potential difference in MPa, R the segment resistance in $\mathrm{MPa}^{\prime} \mathrm{sg}^{-1}, \Phi$ the water flow in $\mathrm{kg} \mathrm{s}^{-1}, \Delta \mathrm{V}$ the part of the water content of the segment that contributes with the water flow in $\mathrm{kg}$, and $\mathrm{C}$ is the hydraulic capacity in $\mathrm{kg} \mathrm{MPa}^{-1}$. From this system HYDRO calculates the hydraulic capacity of each element. The input to the model is simulated by a resistance value and a capacity value that represent the soil-root system.

The water flow is calculated in two iteration steps. The first iteration calculates the water flow adding the transpiration of each leaf element without taking into account the reserves in the xylem. Like most models for water transfer, evapotranspiration $(\mathrm{E})$ is the driving force of the hydraulic system. Through the water loss a water deficit results in the system. This water deficit is compensated by drawing off water from lower parts of the plant, until the soil is reached. The model assumes that the needed amount of water is directly absorbed from the soil. The system must be initialised by adding the evapotranspiration values of all the carried leaves as in the formula:

$$
\Phi_{i}=\sum_{j=1}^{j=n} E_{j}(t)
$$

where each element $i$ carries $j$ leaves. 
Through the interaction between the hydraulic resistance of the soil-root system and the ground water potential a specific hydraulic potential results at the root collar. The hydraulic potentials along the hydraulic system are then calculated starting from the root collar potential until each leaf has been reached $\left(\Psi_{\mathrm{t}}\right)$. With this new generated set of hydraulic potentials a second iteration is started. It calculates anew the water flow, this time taking into account the water reserves of the xylem and of the root system. The difference of the actual potentials and the potentials of the previous iteration $\left(\Psi_{\mathrm{t}}-\Psi_{\mathrm{t}-1}\right)$ or $\Delta \Psi$ is used in 2-32 to calculate the changes in the water reserves of each element and thus the change of the flow through each of them. The iterations are repeated until given convergence criteria are fulfilled. Figure 2-11 shows the iteration stages.

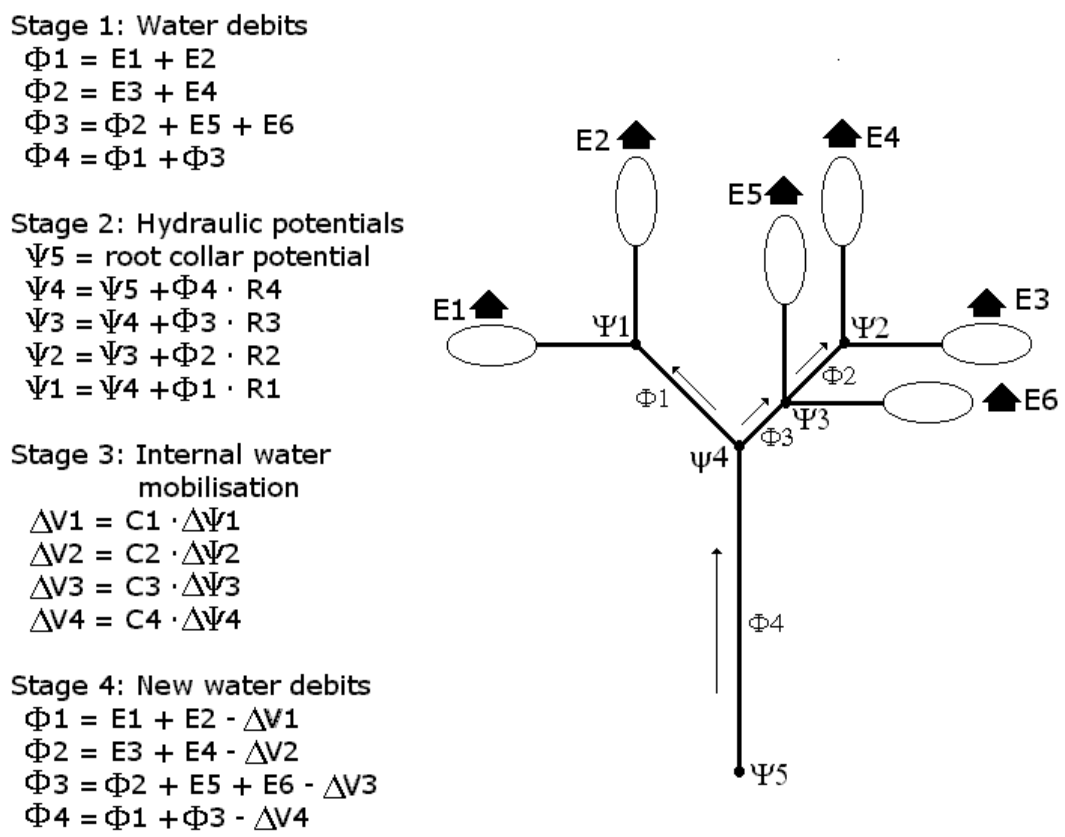

Repeating 2-4 until convergence criteria reached

Figure 2-11: Function schema of the water flow model HYDRO from J. Dauzat. Ei is the evapotranspiration of the leaf element $i$ used to calculate the water deficit $\Phi$ n of each segment $\mathrm{n} . \Psi \mathrm{j}$ is the hydraulic potential at point $\mathrm{j}$, where $\Psi 5$ is the potential at the root collar. $\Delta \mathrm{Vn}$ is the change of the water reserves, $\mathrm{Rn}$ the hydraulic resistance and $\mathrm{Cn}$ the hydraulic capacity of segment $n$ (After RAPIDEL, 1995).

In contrast to HYDRA, HYDRO calculates only an equilibrium state of water flow dynamics. No dynamic simulations concerning short-term reactions of the hydraulic system, e.g. the answer to a sudden increase in transpiration, are possible. The numerical properties of the iteration algorithm of HYDRO were not so carefully checked on mathematical grounds than 
was done for HYDRA. On the other hand, HYDRO was much closer linked with microclimatic models (simulating radiation and energy balance) from the beginning on. Therefore it makes sense to try to combine the advantages of both models.

\subsection{Structure models}

The range of structure oriented modelling approaches is very vast, reaching from simple tree shape models up to models describing single leaves and branches. There exists also models for single trees and for whole stands, the later being an important tool for forest management.

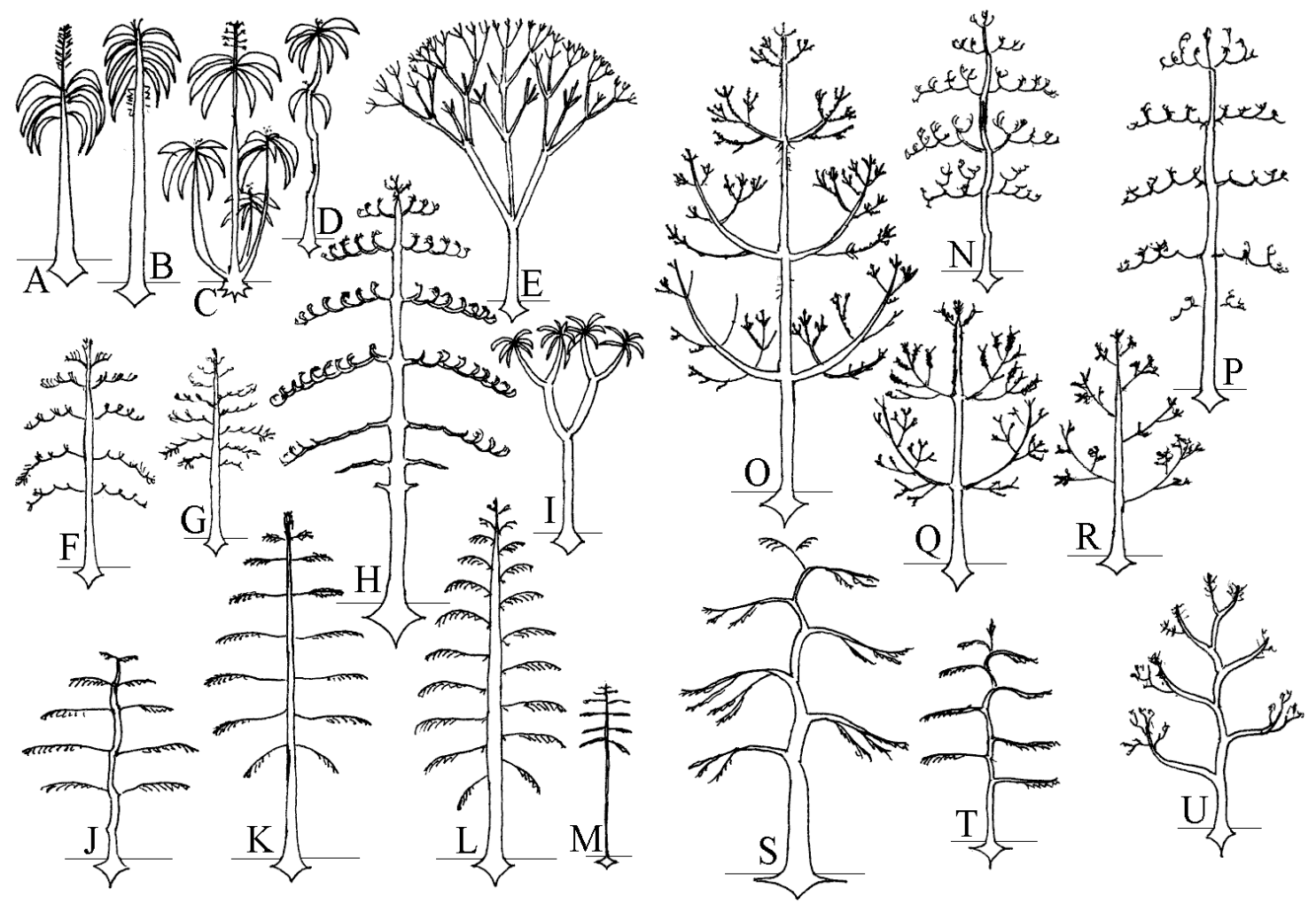

Figure 2-12: Architecture models proposed by Hallé and Oldeman. A-Holttum, B-Corner, CTomlinson, D-Chamberlain, E-Leeuwenberg, F-Fagerlind, G-Petit, H-Aubréville, ISchoute, J-Nozeran, K-Massart (Abies, Ilex), L-Roux, M-Cook, N-Koriba, O-Rauh (Pinus, Acer), P-Prevost, Q-Scarrone (Aesculus), R-Attims, S-Troll (Fagus, Tilia), TMangenot, U-Champagnat (Prunus, Rosa). After Richards, 1996. Not represented are the models McClure and Stone

\subsubsection{Basics}

Hallé and Oldeman (Hallé and Oldeman, 1970; Hallé et al., 1978; Hallé, 1986) developed a system of "models" or architectural schemes based upon a series of morphological 
characteristics and growth patterns. All plants could then be placed in one of 20 models thus allowing the development of a more reductionist view of forest complexity (Tomlinson, 1983). The different models are named after renowned botanists and show the increasing complexity of growth forms from simple angiosperms, like Corner and Chamberlain, to the taxonomically higher order families with complex branching patterns, arrangements of flowers and a reduction in the size of vegetative and reproductive structures, like Massart, Troll and Rauh. These models are based on the external harmony of the tree shape according with specific growth patterns. Figure 2-12 shows the models proposed by Hallé.

This system is more a classification tool than a simulation tool. Some trees cannot be clearly ascribed to one category, but are intermediate between some of them. Even other trees change from one model into another during different growth stages. However, the principles of growth described here can be used as a base for more complex architecture models. The growth of plants can thus be simplified into only few growth rules. The implementation of such rules is the primary objective of most models.

Descriptive models concentrate on single or few rules for growth. Some criteria used to describe branching systems are the bifurcation ratio $\left(\mathrm{R}_{\mathrm{b}}\right)$ between branches of different order, the growth direction of stem and branches or tropism (plagiotropy $\leftrightarrow$ orthotropy), the duration of the growth phase (bound or seasonal $\leftrightarrow$ free $\leftrightarrow$ continuous) and the position of the reproductive organs (terminal $\leftrightarrow$ lateral). As with the models proposed by Hallé, these values are often very variable within the same species, and even in the same tree, so that they only offer a guide for further modelling. The orientation of branches (and thus leaves) is also an important characteristic of tree growth. Three main forms are considered: opposed, disperse and alternate according to the divergence angle between buds (opposed = bud pairs rotate $90^{\circ}$, alternate $=180^{\circ}$, disperse neither $90^{\circ}$ nor $180^{\circ}$ ). In this context, maximum divergence is reached with the angle $137^{\circ} 30^{\prime}$, i.e. the exploitation of the available space is maximal.

Purely descriptive models are based on botanical observations: regular branching patterns, distribution of buds along an axis, tropism, reiteration, etc. Reiteration is the replication of the branching pattern of the whole plant at branch level. Reiteration can be an adaptive process, a reaction to special environmental events as observed on Picea abies and other woody plants 
(Gruber, 1987), or it can be part of the natural growth process, and thus it can be characteristic for the general appearance of the plant as observed in some herbal plants, particularly ferns and tropical trees (Kurth, 1999). One could speak of a smaller plant growing in another bigger plant. The mathematical description of reiteration represents a great simplification of the growth rules and is defined by the principle of self-similitude as an extension of the symmetry principle (Kurth, 1999). It is thus only necessary to define the ground pattern, and to repeat it to get more complex structures. Size, position and orientation can vary, but angles and length relations are constant, and thus unrealistic. Plants generated this way tend to look artificial, because they appear too regular. Although these models are based on general botanical observations their realisation concentrates on graphical representation. Only a specific part of the botanical phenomena can be reproduced with self-similar structures. However, these methods provide some very important basics for more complex models (Kurth, 1999; Prusinkiewicz et al., 2001) allowing to simplify growth algorithms beyond the modelling of true reiteration.

\subsubsection{Hydraulic architecture}

As described in section 2.1.7, more complex models for water flow rely on an accurate representation of plant architecture. The structure of plants, especially of trees, must be hydraulically stable, i. e. the water transfer from the roots to the canopy must be granted. The term "hydraulic architecture" was coined by Zimmermann (cited by TAUSEND ET AL., 2000) and describes the hydraulic conductivity of the xylem in relation to the supported leaf mass that it must supply with water. Chiba (CHIBA, 1990; CHIBA, 1991; CHIBA, 2000; CHIBA AND SHINOZAKI., 1994; CHIBA ET AL., 1988) expanded the model of Shinozaki (SHINOZAKI ET AL., 1964) to develop a descriptive model of tree crowns. The branching patterns had to maintain the conditions of the pipe model theory. FARNSWORTH AND VAN GARDINGEN (1995) tested the branching patterns of Sitka spruce against the pipe theory, and Anzola (KURTH AND ANZOLA, 1997; ANZOLA, 1998) developed a growth model for Picea abies which takes into account the principles of the pipe model theory. OPPELT ET AL. (2001) tested the topology of root systems from three African tree species under consideration of Leonardo's rule. GAFFREY AND SLOBODA (2001) presented some propositions for modelling tree morphology in dependence upon its hydraulic characteristics. The models HYDRA and HYDRO described in sections 
2.1.7.1 and 2.1.7.2 offer a possibility to link growth engines to generate trees that are conform to the requirements of hydraulic architecture. This, however, has not been yet realised.

\subsubsection{Mechanical models}

While the pipe model theory seems to explain the phenomenon of secondary diameter growth of woody plants very well, some growth events at the base of the stem and of branches are beyond the model of Shinozaki. Mechanical stress has an influence on growth in diameter. Mechanical stress can result from the need of supporting the own weight, especially by branches of first order or higher, from wind stress and as a reaction to irregular events like losing the apical bud and the consequent raising of a lateral branch to take on its position, or ice and snow loads. Mechanical stress can then lead to an increased diameter growth. The description of elasto-mechanical characteristics of trees usually concentrates on bending and torsion (GAFFREY AND SLOBODA, 2001). Diameter growth resulting from mechanical stress is adaptive, i.e. a reaction (adaptation) to a particular stress situation. However, it is important to differentiate between stress and strain. While stress can be relatively constant (e.g. predominant wind direction), strain is subject to permanent changes due to the growth process. The natural growth process can lead to mechanical stress. MATTHECK (1993) describes three internal regulators responsible for possible mechanical stress. First, through apical dominance the lateral branches are forced to grow away from the main stem, so that the apical branch gets better growth conditions. Second, through negative geotropism every branch tries to grow vertically. The contrariety of both forces must be compensated to minimise the mechanical strain. And third, phototropism can induce bending growth when the light distribution is not optimal and the branches are forced to growth in deviation to the ideal vertical direction. Because wind is the most important external factor of mechanical stress, the significance of the wind models presented in section 2.1.6 has increased considerably, together with their interconnection with detailed crown models (MöHRING, 1980). MENCUCCINI ET AL. (1997) investigated hydraulic and mechanical characteristics of tree structure of Scots pines at different ages to describe the interaction between hydraulic and mechanical properties during plant growth. MCMAHON AND KRONAUER (1976) described tree growth under application of an optimality principle with respect to carrying the own weight. They defined "elastic self-similarity" of tree branches as a fairly constant value of an exponent in a power law relating diameters and lenghts. Loup (LOUP ET AL., 1991) 
investigated the relationship between architecture, mechanical properties and anatomy of Pinus pinaster Soland. They explain the re-orientation of the meristems according to mechanical needs after traumatic events, and the anatomical changes that result in this process. FARNSWORTH AND VAN GARDINGEN (1995) searched for a pattern in secondary diameter growth in relation to both hydraulic and mechanical principles. They concluded that mechanical laws have a higher explanation power than hydraulic needs for the patterns they observed in their sample trees. GAFFREY AND KNIEMEYER (2001) developed a threedimensional model to simulate the elasto-mechanical reactions of trees to wind and snow loads and tested it on a 64 years old Douglas fir.

\subsubsection{Forest models and the transition to functional-structural models}

Classic forest models like yield tables and height curves are no more accurate enough to predict the growth behaviour of plants upon changing environment and upon modern ecological forest management. Modern models are based on general applications of growth functions (e.g. MURRAY AND VON GADOW, 1990) or on the analysis of the stand structures to reproduce them (PretZsCh, 1990a; Pretzsch, 1990b; LEWANDOWSKI AND VON GADOW, 1996; Pommerening, 2000). These models, however, lack the information needed to explain accurately more complicate processes. Thus, models which provide a well suited basis for further modelling had to be created.

\subsubsection{Single tree based models}

Different architecture models based on the structure of single trees have been developed. These models can also be used to represent whole stands by generating a number of single trees. The main flaw of most models of this kind is that they are often developed to describe a single species or even a single sub-species. The re-adjustment for other species is very difficult or even impossible. However, these models provide important basic knowledge for plant modelling. Most older models were limited to single or few parts of plants and to the growth behaviour of meristems.

RENSHAW (1985) developed a stochastic growth model of the canopy and the main root system for Sitka spruce (Picea sitchensis) based on data of 39 15-years old trees. Renshaw 
measured branch length, branching angle, number of daughter branches and orientation, and formulated his model using regression analysis. He simulated temporal dynamic by including time dependant variance of growth parameters, and demonstrated the importance of variance in the growth process. He argued that the choice of variances is more important than the choice of distribution functions.

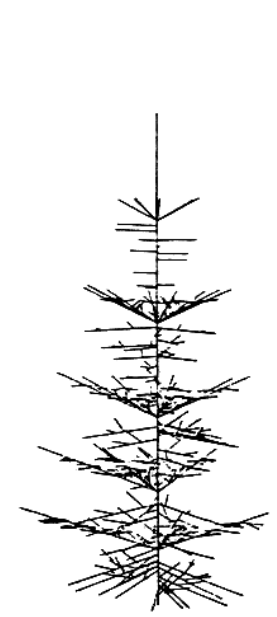

a

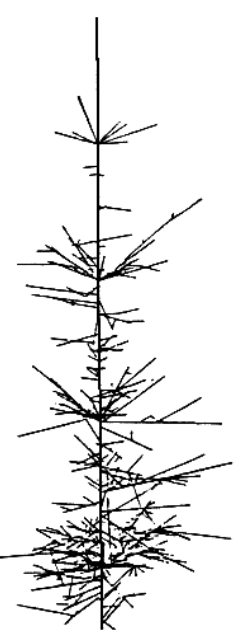

b

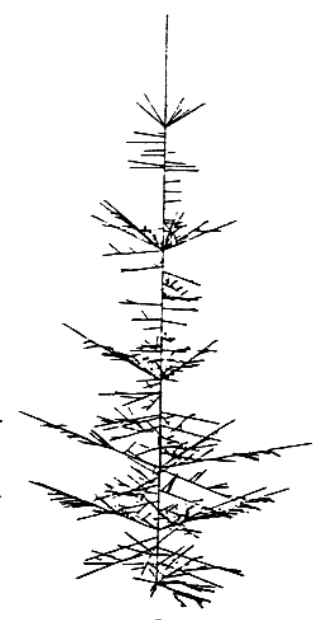

$\mathrm{C}$

Figure 2-13: Representation of a Picea sitchensis tree with the model of RENSHAW (1985). a) Visualisation of original data. b) Tree simulated with high variance. c) Tree simulated with low variance.

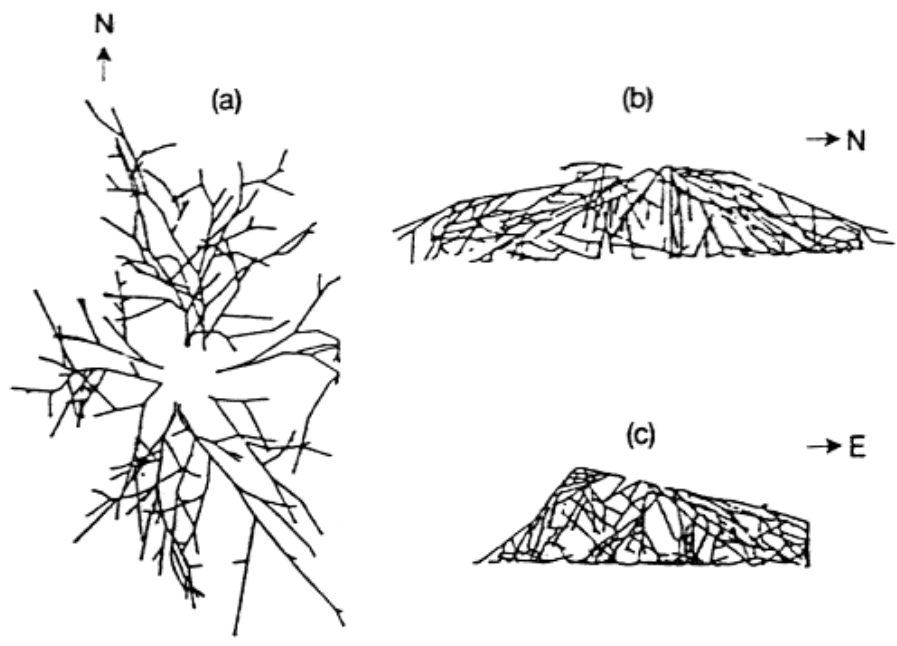

Figure 2-14: Structural root system simulated with the model of RENSHAW (1985): a) view from above, b) and c) lateral views.

Figure 2-13 shows the result of the simulation of a tree canopy using different variance levels, and Figure 2-14 the simulation of a root system. Renshaw also argued that the application of 
the model for stand simulation would not be appropriate due to the independent development of each tree in the stand. BARTÁK AND JANOUŠ (1989) presented a simple architecture model for Turkey oak (Quercus cerris L.) based on data of an approximately 80 years old tree. The data was limited to $1^{\text {st }}$ order branches and to three branch samplings to investigate the orientation of leaves. Realistic simulation of tree crown was not achieved.
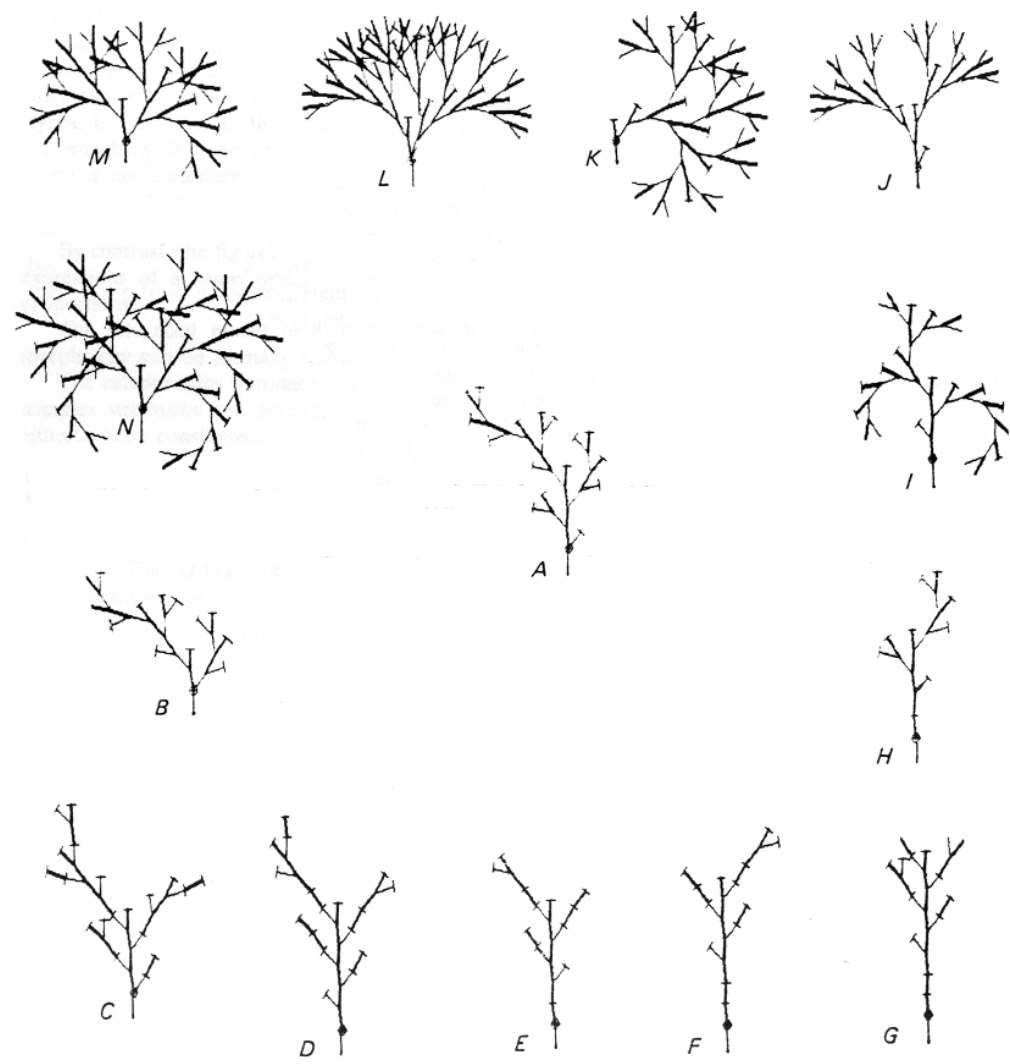

Figure 2-15: Simulation of 14 branching systems (A-N) with the model of LÜCK ET AL. (1990) using different parameters. The systems from A to $\mathrm{H}$ correspond to the determinate growth of tomato (Lycopersicon spc.), with acrotonic branching. The systems I to $\mathrm{N}$ correspond to indeterminate growth.

LÜCK ET AL. (1990) developed a generic model to describe acrotony, mesotony and basitony in plant growth. The model is based on a parallel rewriting method similar to the L-systems presented below. However, the branching rules are represented by equations and not by grammars. Growth is regarded as a bifurcation process. The main bifurcation characteristics are described by monopodial or sympodial growth for the local characteristics and by basitony, mesotony and acrotony for the global characteristic. The model was tested on Lycopersicon spc. (Tomato) plants. Figure 2-15 shows some simulation results using a range of parameters to describe determinate and indeterminate growth. 
LEDIG (1991) created a visualisation software for Norway spruce (Picea abies (L.) Karst.), which, like the model of Barták and Janouš, is limited to $1^{\text {st }}$ order branches. The program was a graphical tool to determine wood quality in dependence of limbiness, and was not really a model. It shows, however, the application possibilities of even simple tree growth models. The interconnection of standard growth models with such tools would be a significant expansion.

KELLOMÄKI AND KURTTIO (1991) developed an empirical growth model for Scots pine (Pinus sylvestris). The model describes the crown dynamics based on the growth of single branches in dependence on the characteristics of the respective mother branch. The model is based on data of young trees (7-18 years) from different plantations in Finland, measuring for each branch length, diameter, branching angle, biomass, needle density and number of daughter branches. Although the data for the model comes from young trees, the model can simulate much older trees. By this time, the model has been expanded to take into account the local effects of light on growth (KELLOMÄKI AND STRANDMAN, 1995; KELLOMÄKI AND IKONEN 1996).

a)

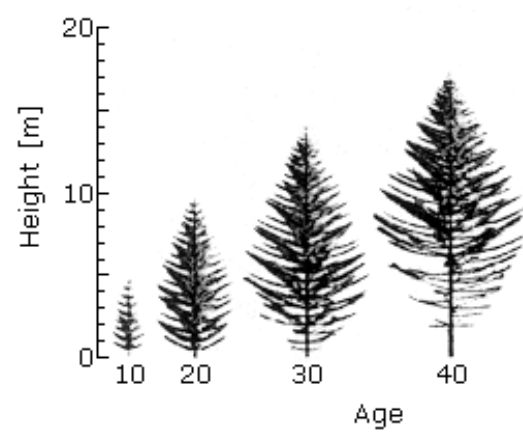

b)

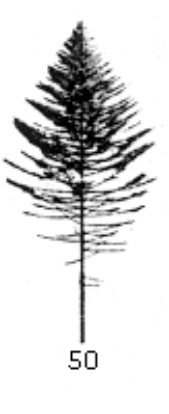

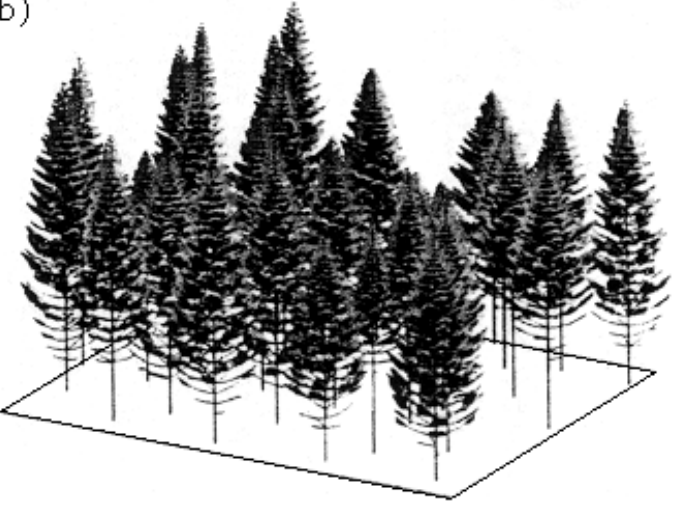

Figure 2-16: a) Simulation of a single Abies sachalinensis tree at different ages. b) Simulation of a 35 years old stand after thinning. After SUZUKI ET AL. (1992).

SUZUKI ET AL. (1992) developed a similar growth model for Todo fir (Abies sachalinensis). The model includes the bending of lateral branches, growth cessation and regeneration. The model is devoted to the visualisation of tree shapes and tree stands. The equations used for the simulations are based on basic biometrical equations from classical forestry for height and diameter growth. Figure 2-16 shows different stages of growth of a tree every ten years and the final stage of the simulation of a 35 years old stand after thinning $50 \%$ of the trees. 
DORUSKA AND BURKHARD (1994) modelled the diameter distribution of branches and their location in the crown of Loblolly pine (Pinus taeda L.). The model is limited to equations without graphical representation of the resulting structures. WOODCOCK ET AL. (1995) modelled the changes of crown morphology and branching patterns of beech trees (Fagus sylvatica L.) caused by disease syndromes not attached to known pathogens (decline). This model is also limited to the description of the branching patterns of first order branches in form of equations. DELEUZE ET AL. (1995) developed a model for the crown shape of Picea abies based on branch length increment and inclination. The model is concentrates on height growth and is limited to young trees. The list of this kind of models is long. The knowledge gained from models describing plant growth with a set of equations can be used in models that permit the simulation of different species based on general parameter definition or on abstract modelling languages. More detailed models - often aiming at graphical representation of tree structure - can provide more accurate information but are often difficult to combine, compare and expand with other models.

\subsubsection{Lindenmayer Systems and GROGRA}

LINDENMAYER (1968) developed a formalism for modelling filamentous organisms based on parallel string rewriting. The general approach got the name Lindenmayer- or L-Systems. LSystems can be used for the modelling of tree structures and their growth, through the description of their geometry and its development in time. The representation of tree structure consists of a series of concatenated symbols called string and includes the topological and geometrical information of each element (see section 1.2) and optionally also other, nonstructural attributes as colour, leaf mass, chemical composition, etc. (KURTH, 1999). The modelling of the temporal development of structures is achieved through the application of rules which describe how the structure may change from one time step to another. L-systems consist of the following elements:

1. A set $\Sigma$ of symbols (alphabet) for the representation of structure elements and operators.

2. An initial structure represented by a start symbol or start string (axiom) $\alpha$ consisting of symbols from $\Sigma$. 
3. A set of rules for the rewriting of strings. These rules are applied to every symbol in the string at time $t$ to generate a new string at time $t+1$. Symbols that do not match any rule remain unchanged. These are called generative rules, and take the form:

$$
\text { symbol -> string of symbols }
$$

4. For the (graphical) representation of the string, it must be interpreted and translated into a spatial structure. This process is called geometrical interpretation or semantic.

The application of the generative rules to the string is normally iterated many times. The string at each iteration step represents a plant at different stages of its development. For each time step $t$ or generation, a string $\sigma_{t}$ is generated, where $\sigma_{0}=\alpha$. For most simple models, each time step corresponds to one year, which is reasonable for trees and other long term simulations. For the modelling of other processes like the development of flowers and fruits or leaf fall in autumn other temporal scaling can be used. The geometrical interpretation is implemented using turtle geometry (ABELSON AND DISESSA, 1981). Turtle geometry was developed for the programming language Logo in order to control a graphical device called "turtle". The first "turtles" were mechanical devices carrying pens to draw on paper on the floor. In modern applications the turtle is a virtual device that helps to draw structures on a monitor. Turtle geometry describes paths "from within" rather than "from outside". For example, "turn right" means turn right relative to the original heading direction of the turtle and not relative to an absolute reference point. The user manipulates the graphical turtle by telling it to move forward or back some number of steps, or by telling it to turn left or right some number of degrees. The advantage of turtle geometry is that many paths are more simply described in relative than in absolute terms. In L-Systems, the virtual "turtle" is controlled by a subset of the alphabet $\Sigma$. The subset of commands can vary in complexity and size (compare PrusinKIEWICZ AND LindenMAYeR, 1990; KURTH, 1994; KURTH, 1997; KURTH, 1999). The interpretation of the string $\sigma_{t}$ produces a geometrical structure $\mathrm{S}_{t}$. The development of an L-system is represented in Figure 2-17, where the horizontal arrows represent the generative rules and the vertical arrows represent the geometrical interpretation. KURTH (1999) describes three main levels for the description of geometric plant structures (Figure 2-18): The representation of a structure at a given time, the definition of rules for the development of the structure, and the possibility of controlling the dynamics of development by choosing between different applicable rules. 


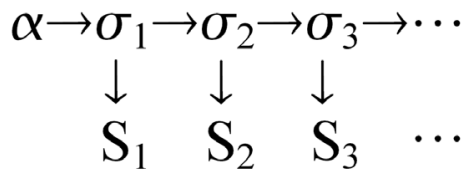

Figure 2-17: Working scheme of an L-system. $\alpha$ is the initial string, $\sigma_{t}$ the string at time $t$, and $\mathrm{S}_{t}$ the corresponding geometrical structure. Horizontal arrows represent the application of generative rules, vertical arrows the geometrical interpretation. After KURTH, 1999.

The concept of L-System and their application for plant modelling have been used and expanded by many authors to include for example stochastic rules (NISHIDA, 1980), parametric grammars (LindeNMAYER, 1974; HERMAN AND SCHIFF, 1975; CHIEN AND JÜRGENSEN, 1992) and context sensitivity (PRUSINKIEWICZ AND LINDENMAYER, 1990). The use of all these expansions have made of the original L-Systems a powerful modelling tool capable of going beyond simple structure models, and thus enabling the creation of structuralfunctional models that can be combined with different graphical tools for impressive graphical simulations (KURTH, 1994; KURTH, 1997; KURTH, 1999; KURTH, 2002; KURTH AND Anzola, 1997; Kurth And FrÜh, 1998; Kurth AND SlobodA, 1997; KuRTh AND Sloboda, 2001; MĚch And Prusinkiewicz, 1996; Prusinkiewicz, 1987; Prusinkiewicz, 1994; PrusinkiewicZ, 1997; PrusinKIEWICZ AND LindenMayer, 1990; PruSinKIEWICZ ET AL., 1998; PrusinKIEWICZ ET AL., 1994a; PrUSINKIEWICZ ET AL., 1994b; PrUSINKIEWICZ ET

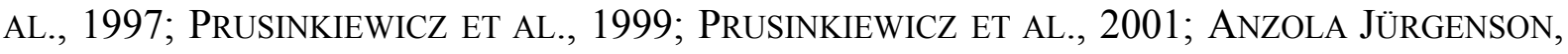
1998).

In context sensitive systems the application of generative rules depends not only on the respective symbol but also on the neighbour symbols. PRUSINKIEWICZ AND LINDENMAYER (1990) applied context sensitivity to simulate hormone activity in plants. The inclusion of stochastic rules in L-systems permits to control the application of generative rules (Figure 2-18c). Rules can be provided with a probability value, and then a rule is selected randomly and applied to the symbol. The selection is made for each instance of the symbol separately. Further, stochastic L-systems permit the simulation of Markov chains for modelling meristem state transition (MAILLETTE, 1990; GUÉDON, 1997). In parametric L-systems each symbol can be equipped with a list of real-valued parameters defining attributes like age, vitality or Creserves. 
The software GROGRA developed by KURTH (1994) at the University of Göttingen is a growth grammar interpreter based on L-Systems. GROGRA offers different possibilities for (1) structure generation based on different kinds of L-systems, (2) reading pre-generated structures, (3) analysis of a given structure under different criteria, (4) converting data from and into other data formats (AMAP, HYDRA, etc) and (5) graphical representation of structures.
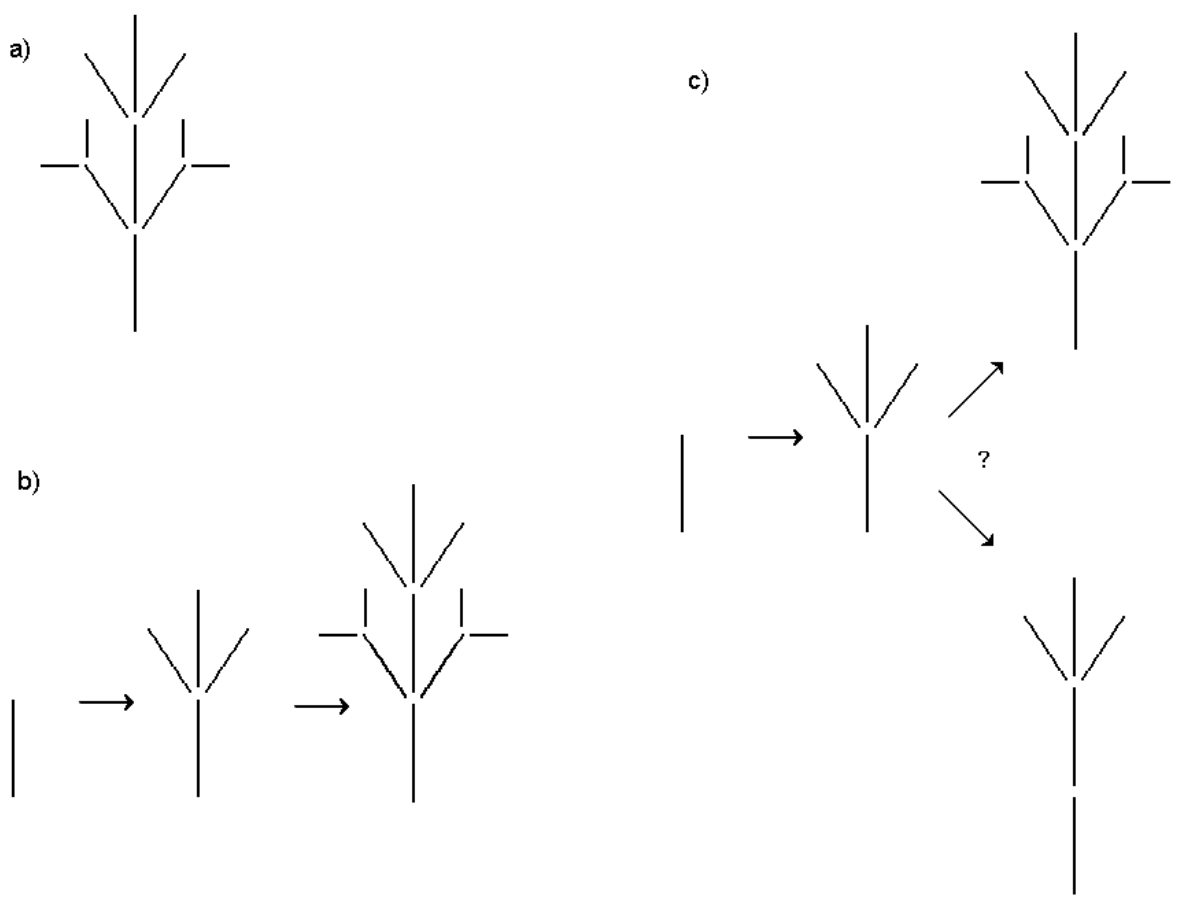

Figure 2-18: Three levels of the formal description of plant structures. a) static geometrical description. b) Development of a structure over time as achieved by basic L-systems. c) Control of the development as achieved with stochastic or sensitive L-Systems. The arrows represent the application of generative rules (after KURTH, 1994).

L-systems are written in two formats, $l s y$ for non-sensitive and $s s y$ for sensitive L-systems. In this context, sensitivity means more than context sensitivity as described above. Kurth refers to it as global sensitivity, where both the structure $\mathrm{S}_{t}$ and its environment can exert influence on the generative rules. Further, both formats include the possibility to define parameter values for each symbol, and thus, they support parametric L-systems. For more details about the language formalisms for L-systems in GROGRA see KURTH, 1999. Although the software is written in the programming language $\mathrm{C}$, knowledge about $\mathrm{C}$ is not required to use GROGRA. Only the syntax for arithmetical expressions is the same as in C. However, GROGRA also provides the possibility to call pre-defined functions in L-systems. These C- 
functions are included in the source code and cannot be changed by the user. The functions and their respective code are listed in KURTH, 1999.

For the description of structures, GROGRA provides the formats $d t g$ for the most explicit description of generated structures, $d t d$ (descriptive tree data, see 3.1.3) for the description of empirically mapped tree structures, $d t b$ for data exchange with AutoCAD, $p b g$ and $s b g$ for data exchange with HYDRA. Furthermore, GROGRA can read and generate linetree files for data exchange with AMAP. As shown here, GROGRA provides a vast range of interfaces with other models and tools, and thus it is well suited as a base for model interconnection. KURTH (1999) describes the possibilities of GROGRA and demonstrates the "universality" of L-systems by reproducing existing plant models from the literature, as e.g. the light model of TAKenAKa (1994) and the model LIGNUM (PERTTUNEN ET AL., 1996). GROGRA is per se not a model, but a growth engine which implements single models in form of L-systems. The range of models that can be implemented this way is very vast and not limited to structural models. The application of the advanced features of L-systems - stochastic and parametric systems - allows the implementation of structural-functional models based for example on light distribution, spatial competition, mechanics and allocation of assimilates.

\subsubsection{AMAP}

The modelling software system AMAP (Atelier de Modélisation de l'Architecture des Plantes) was developed by the team of Philippe de Reffye at CIRAD in Montpellier, France (BARCZI ET AL, 1997 ; DE REFFYe ET AL., 1991; DE REFFye ET AL., 1995a; DE REFFYE ET AL., 1995b; De RefFye Et AL., 1997; LeCoustre AND De RefFye, 1993). Similar to GROGRA, AMAP is not a model per se but a growth engine (moteur de croissance).

In AMAP, plant modelling is based on the description of the state of meristems. De Reffye describes three basic states of a meristem: dormancy (état de dormance), growth (état de croissance) and death (état de mortalité). The transition between these states follows stochastic laws specific for each species and even sub-species. Compare in this context the application of stochastic L-systems as described above. For the description of plant growth, especially of periodic plant growth, a basic element called growth unit (unité de croissance) is defined as the product of a meristem in a growth period after the elongation phase. The 
elongation phase includes the complete growth process, and thus is finished after the eventual neo-formed growth. In general, a growth unit corresponds to a shoot and includes a set of internodes, nodes, leaves and buds produced in this time. For the modelling of growth it is important to describe the number of internodes in a growth unit in dependence on age, situation, branching order, etc., as a function of the productivity of meristems. The distribution of the number of internodes is characteristic for the random growth of shoots. If the meristems follow only pre-formed growth, the distribution is unimodal, as in red beech (Fagus sylvatica). By neo-formed growth ("Johannistrieb") the distribution is bimodal, as in black cherry (Prunus avium). Within a tree, the distribution for equivalent vegetative axes (same branching order and physiological age) is similar (Figure 2-19). Such investigations have been made for a series of agricultural and forest plant by the team at CIRAD.

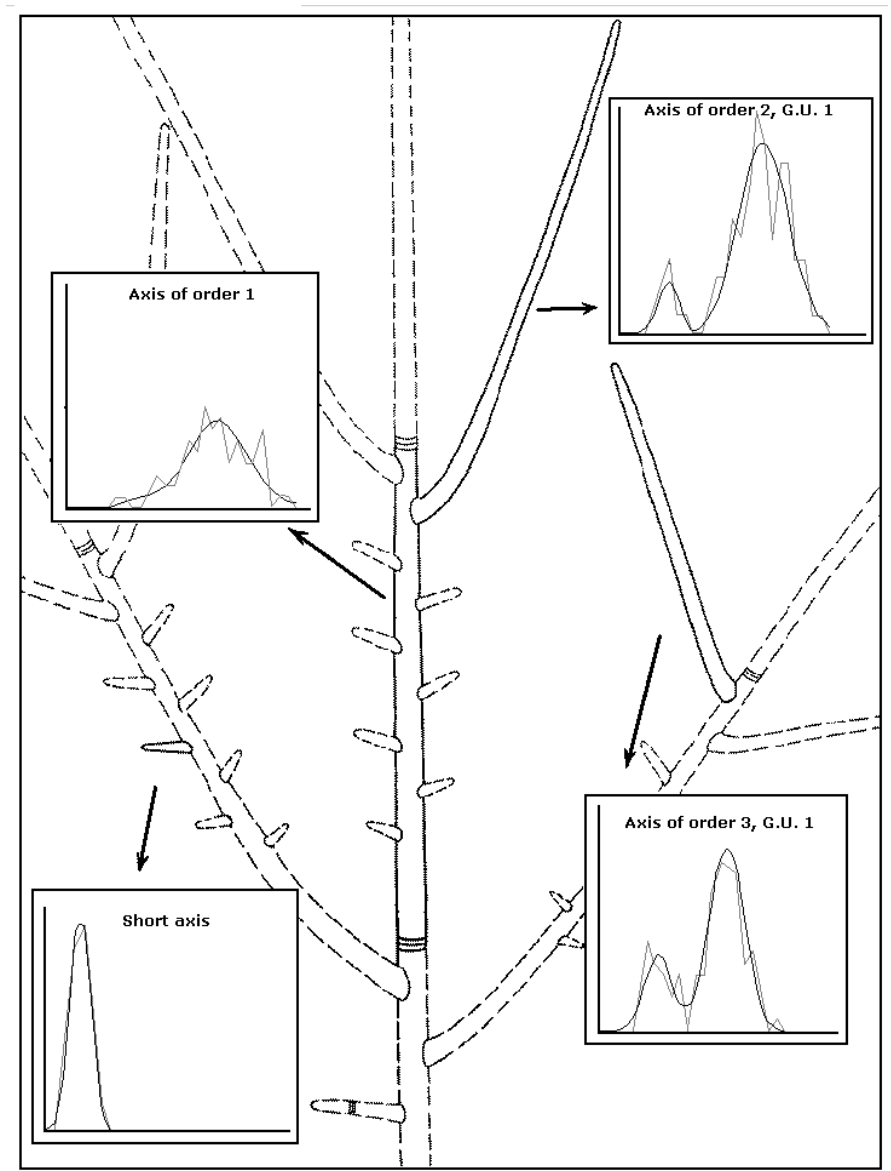

Figure 2-19: Frequency distribution of number of internodes per growth unit at different branching orders and modalities in black cherry (Prunus avium). The axes of order 2 and 3 have pre-formed and neo-formed growth and thus a bimodal distribution. Axes of order 1 and short shoots have only pre-formed growth and thus a unimodal distribution. On the graphics, the horizontal axis is the internode number and the vertical axis the frequency (after DE REFFYE ET AL., 1991). 
For the further description of tree growth, more information is necessary. The branching modality of a lateral meristem becomes significant. The branching patterns are described by the distribution of branched and unbranched zones along the growth unit. This process follows a transition probability from a state into another: unbranched $\rightarrow$ unbranched, unbranched $\rightarrow$ branched, branched $\rightarrow$ branched and branched $\rightarrow$ unbranched, and can be easily described by a Markov chain of order 1 with the two states branched and unbranched (Figure 2-20). Different branching patterns can result in equal global branching frequencies, so that the local probabilities $\mathrm{P}_{1}$ (unbranched $\rightarrow$ unbranched) and $\mathrm{P}_{2}$ (branched $\rightarrow$ branched) are more important for the description of the branching process.
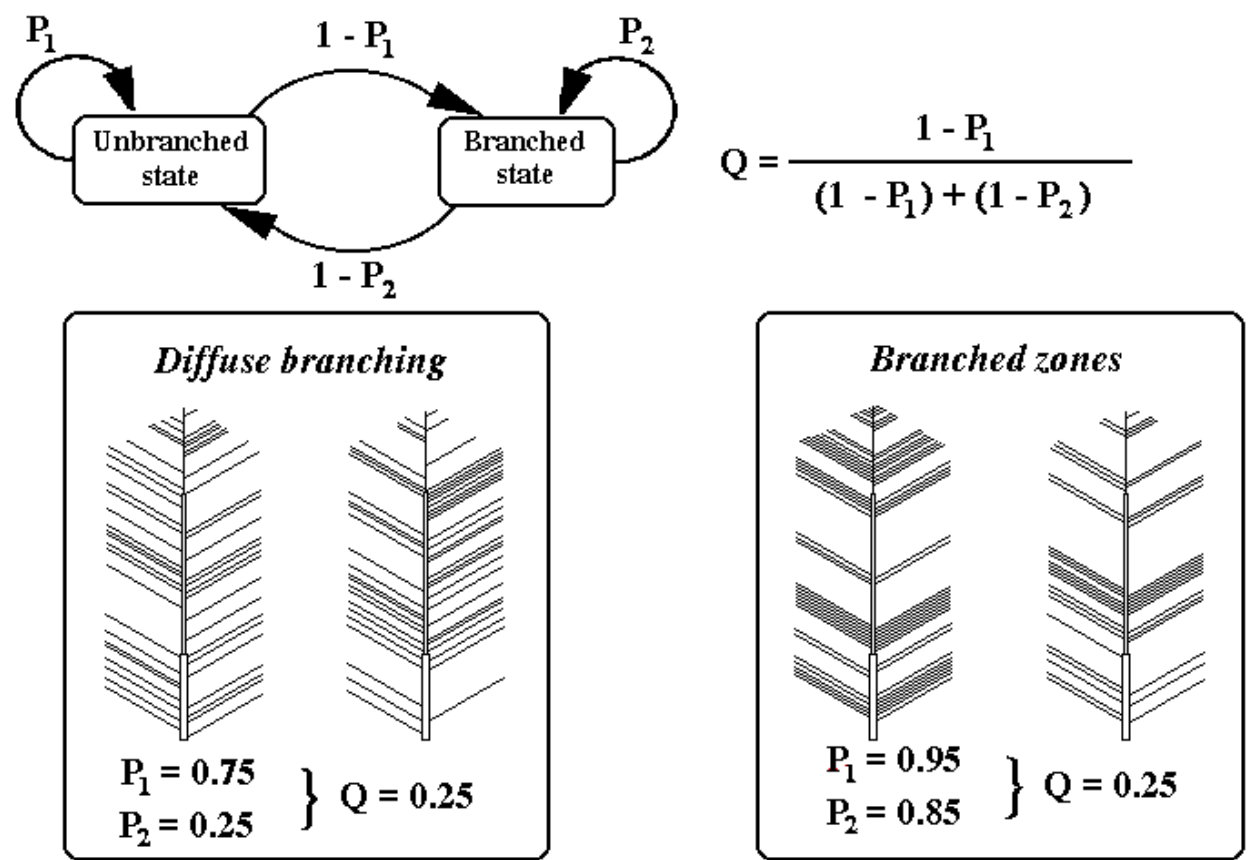

Figure 2-20: Distribution of branched and unbranched zones in a growth unit. The branching process is described by a Markov chain with the transition probabilities $\mathrm{P}_{1}$ (unbranched $\rightarrow$ unbranched) and $\mathrm{P}_{2}$ (branched $\rightarrow$ branched). Q is the global branching probability, and is independent from the distribution itself. With different parameters, diffuse and clustered distributions can be generated.

The form of these distributions is assumed to be independent from the environment. On the other hand, the numeric parameters change by changing environmental conditions and according to increasing physiological age. To describe this change, the term morphogenetic gradient was defined as “... the progressive ageing of the apical meristem of an axis..." (DE REFFYE ET AL., 1995a) (it becomes physiologically old), and “... the progressive increase of vitality in the juvenile phase..." (DE REFFYE ET AL., 1995a) as by reiteration (physiologically 
young meristems). It is possible to group growth units of similar type and function which have all the same physiological age and branching patterns. For each group a theoretical ageing scale or axis can be defined, which contains all the possible stages that a growth unit can adopt. De Reffye called this construct "reference axis" (axe de référence). Bud activity of apical meristems follows a progressive development step by step along the axis with increasing physiological age. In some cases a jump to a higher stage occurs, as by the production of reproductive organs or of short shoots, which represent the end of the reference axis (Figure 2-21). By extending the information content of the axis it is possible to simulate the geometric properties of each element, like length and branching angle.
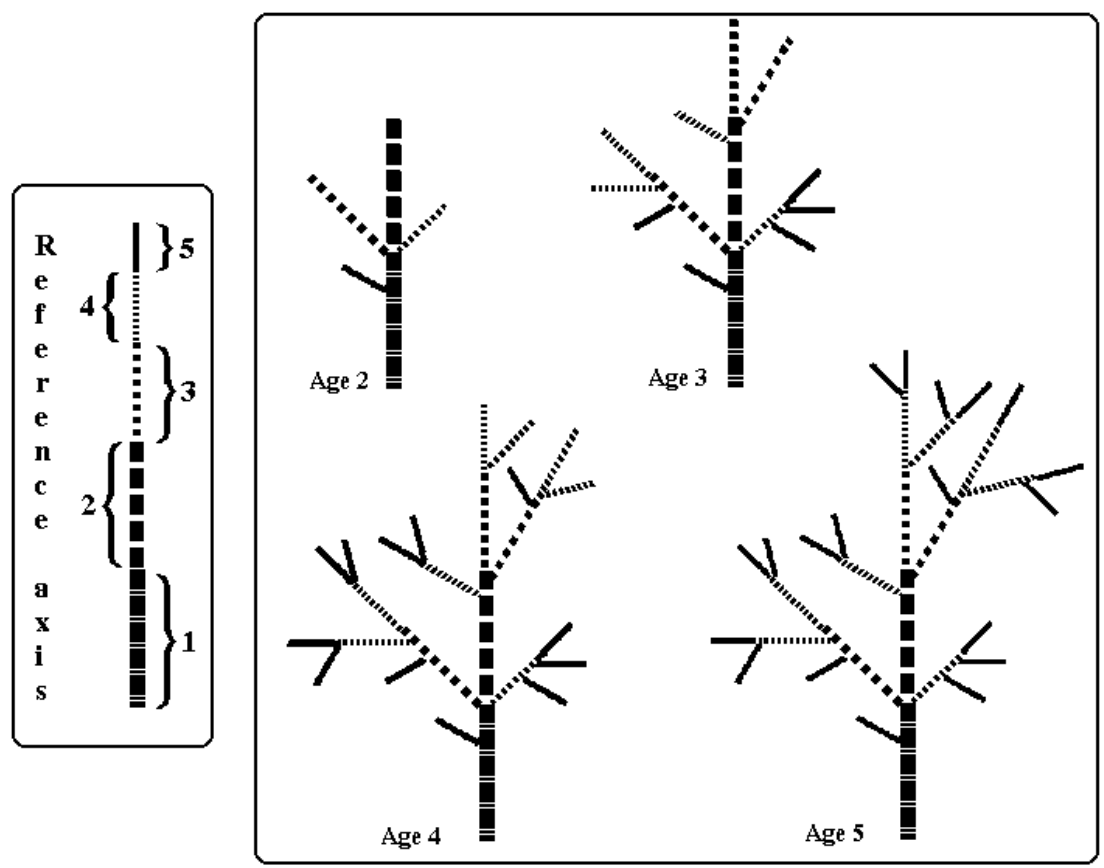

Figure 2-21: Plant growth according to the physiological gradient in a reference axis with five stages. The apical meristem changes gradually from one stage into the next. Lateral meristems can jump to a higher stage, and thus become physiologically older. (DE REFFYE ET AL., 1995a).

To realise the growth simulation using reference axes BARCZI ET AL. (1997) developed the software AMAPsim. It co-ordinates both ageing processes, chronological and physiological, combining stochastic mechanisms represented by a set of topological and stage transition parameters, with geometric parameters in a finite unidirectional automaton. Each axis is simulated from the basis to the end before the next axis is simulated. The result is high detailed three-dimensional plant models that are independent from their environment. AMAPsim is thus a structure model. 
For the simulation of secondary growth, specifically diameter growth, and later to simulate interaction with other plants and the environment the software AMAPpara was created. In contrast to AMAPsim it simulates all the meristems parallel in each stage of growth, and is thus similar to the L-system approach. It was possible with this software to simulate the effect of light interception and spatial competition. Later, the processes for $\mathrm{C}$ allocation were included. This made possible to simulate the effects of silvicultural measures on radial growth. Actually, models including mechanic, hydraulic architecture and pruning have been included. AMAPpara is therefore more than a structure model, it is a structural-functional modelling system.

Additionally, another software called AMAPmod (GODIN, 2000; GODIN AND CARAGLIO, 1998; Godin ET AL. 1999a; Godin ET AL. 1999b) for the analysis of tree structures was developed. The query language AML was created to allow the description and analysis of plants at different spatial scales at the same time. AML is based on the representation of plant structures with multi-scale tree graphs. A tree graph can be compared with a single structuredescribing string used in L-systems (2.2.4.2). There are also other possibilities for the representation of tree graphs (GoDIN, 2000).

Godin proposes using strings to encode tree structures with multi-scale tree graphs. To achieve this, he developed the encoding format MTG. A plant can thus be described at different scales, i.e. the general tree shape as the uppermost scale, and down to the representation of single internodes and nodes. Using multi-scale tree graphs permits to represent different parts of a plant with different scales. For example, the stem could be represented as a unit, while the crown is represented at branch level and reproductive organs at an even more detailed level. This allows to simplify the analysis and to concentrate on important details of tree structures (Figure 2-23).

Besides the topological data innate to tree graphs, the MTG encoding format also includes geometrical information and can be expanded to include further parameters (Figure 2-24). This allows the reconstruction of the plant structure and its representation with 3D graphics. The inclusion of parameters allows, like in GROGRA, the analysis of the tree structure under different aspects: distribution of geometrical parameters, estimated light interception, path lengths for water transfer. This has both benefits and disadvantages. The inclusion of different 
scales in the representation of plants comes along with a higher complexity of the models, and thus possible problems with the understanding. However, the integration of information from different scales in one format is becoming more important in regard to model integration. A compromise between complexity and information content must be met, and this is dependent on the implementation of management tools for MTG.

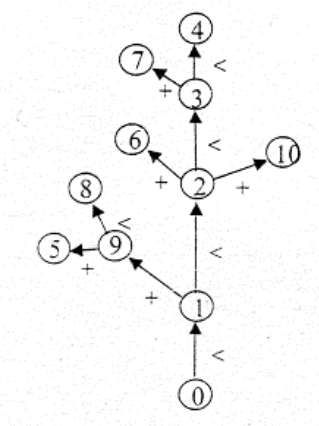

$01[9[5] 8] 2[6][10] 3[7] 4$

Figure 2-22: Representation of a plant with a tree graph coded by a string. The brackets in the string symbolise branching points (GODIN, 2000).
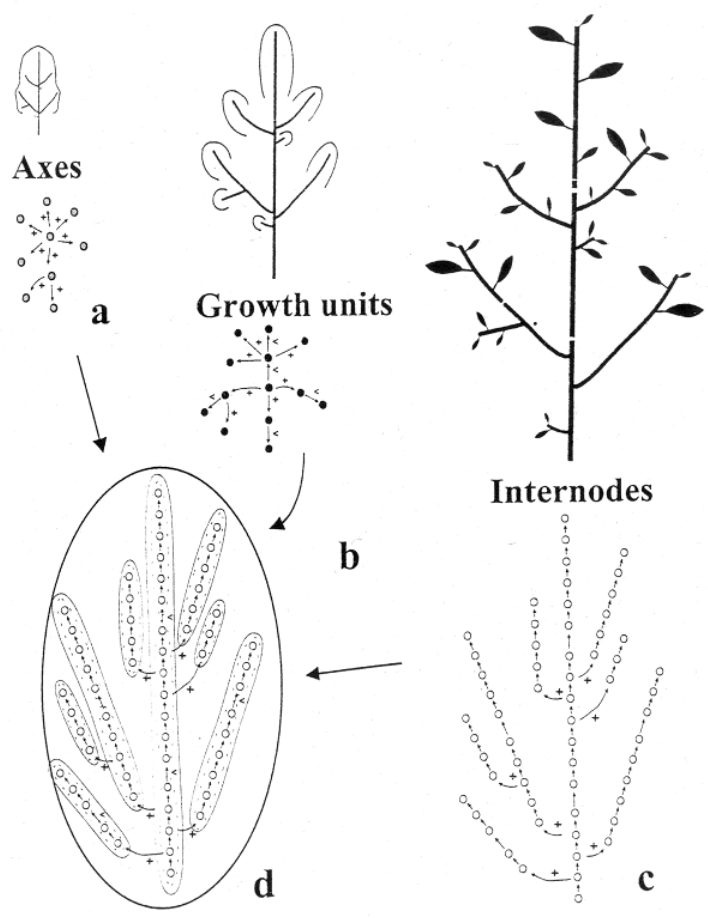

b

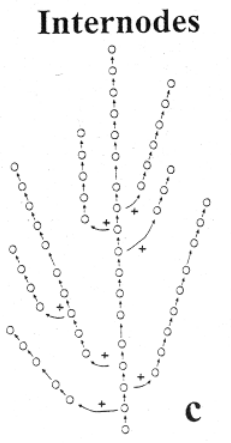

Figure 2-23: Concept of multi-scale tree graphs. A plant can be represented at different scales: a) axis, b) growth units, and c) internodes within a single multi-scale graph d) (GoDIN, 2000).

Most part of the AMAP software is distributed commercially as a graphic software, and is thus difficult to obtain in the framework of many research projects. Further, many aspects concerning the coding of plant structures, parameter files and reference axes are not accessible outside the AMAP group. The development of the MTG format provides a better possibility for researchers and scientists to access the powerful modelling system of AMAP. The software NEXUS presented in this thesis is able to work in a limited form with AMAP formats. But due to the enhanced security procedures of the later versions of AMAP, an interconnection was only possible with the version 2.2 on UNIX systems. Another general problem by the modelling approach in AMAP is the relative inflexibility of the reference axis. 
Even minor changes in branching patterns or reaction rules could implicate major changes in existing reference axes. Also the generation of own parameter files, if allowed, is a very though process. Lanwert (LANWERT, 1997; LANWERT ET AL., 1998) developed a basic program called AIR, which provided a first approach in combining the software AMAPsim with the software HYDRA. Dzierzon (DZIERZON AND KURTH, 2002) used the MTG format to create an interface between AML and LIGNUM.

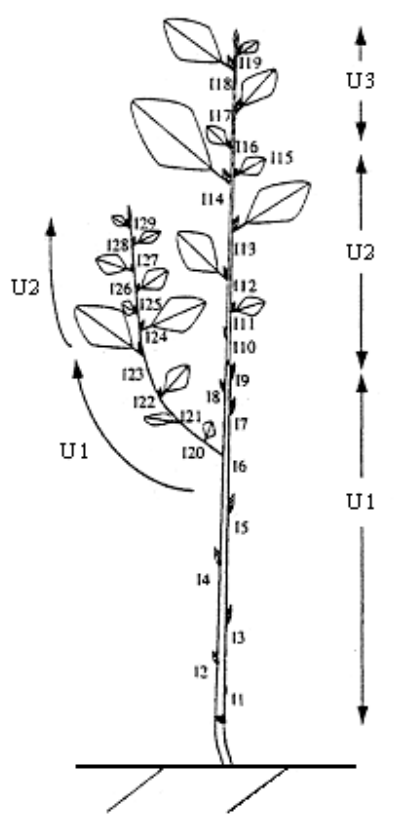

a

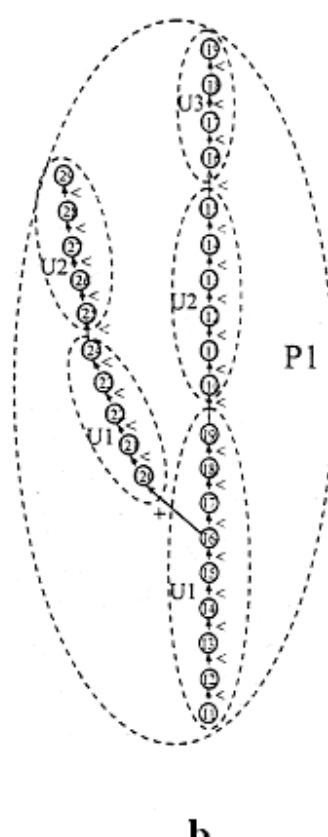

b

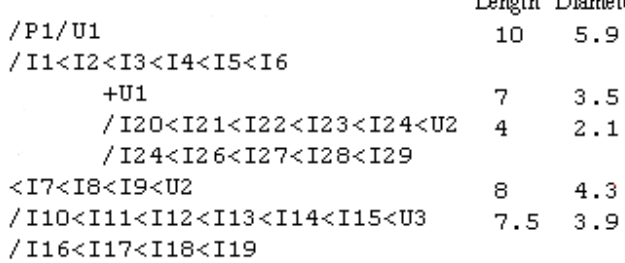

Figure 2-24: Encoding of plant structures with MTG in form of a string. Each scale is given by a slash followed by an indicator. $/ \mathrm{P}$ is the plant scale, /U growth unit scale and /I internode scale. The symbol + means a branching point. Parameters at the end of a line belong to the last element of that line. In this case, growth unit U1 at the base of the plant has a length of 10 and a diameter of 5.9 and the growth unit $\mathrm{U} 1$ at the lateral branch has the values 7 and 3.5 respectively (GODIN, 2000).

\subsection{Functional-structural models}

As described above, both GROGRA and AMAP provide a framework for plant modelling including structure and process oriented models. Most functional-structural models are very complex and combine many of the approaches already described above. However, as with most of the models presented above, these are often very specific, concentrating only on one plant species of type. Furthermore, due to the complexity and extension of these models, it is 
often very difficult for other researchers to gain access to the used procedures, approaches, boundaries, etc. In this section some representative examples for functional-structural models are described, but without the possibility to do it in detail.

AMAPpara, a part of the AMAP software, has to be considered a functional-structural modelling tool. BLAISE (1991) described the parallel modelling of plant growth using voxels, a three-dimensional discretisation of space, to simulate light interception.

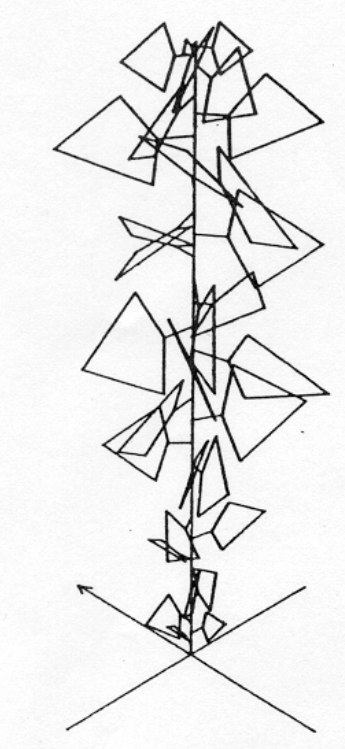

Figure 2-25: Three-dimensional representation of a Populus tree with ECOPHYS (HOST ET AL., 1990).

The model ECOPHYS (HosT ET AL., 1990) is a growth model for Populus trees based on photosynthate production. The model contains several sub-models describing light interception, photosynthate production and allocation. The model is limited to the first year of growth of poplar stem cuttings. Due to the growth condition of the sampling material, water and nutrients are assumed to be not limiting. The model concentrates on light and temperature effects. The trees are represented by a simple architecture model representing leaves as four pointed polygons (Figure 2-25). The light model is based on the sun position, calculated from latitude, date and time of day. The incoming radiation $\left[\mu \mathrm{mol} \mathrm{m} \mathrm{m}^{-2} \mathrm{~s}^{-1}\right]$ is divided into diffuse and direct radiation. For calculating leaf interception, an imaginary plane perpendicular to the light vector was used to calculate the projected area of each leaf and thus shadowing. Direct radiation was supposed to reach the sunlit parts of the leaves. The shaded parts receive only diffuse radiation which is modified by a light transfer curve (see 2.1.4). Photosynthesis and 
respiration are calculated in dependence on light and temperature (see 2.1.5). The model also takes into account the degree of maturity of the leaves to parameterise the used functions. After the carbon fixing in each leaf is calculated, an allocation model is applied to calculate the transport of photosynthates from the leaves to different growth centres. There they are converted into biomass and dimensional growth.

ChIBA ET AL. (1994) use a spherical light model, where the top hemisphere was used to calculate sunlight incidence and the lower hemisphere to calculate reflected light. To calculate light interception, leaves where clustered into leaf-balls, whose radius is proportional to the number of leaves of each branch. Light interception is calculated using the projection of the shadows from other leaf-ball on the light sphere and then running a hidden surface algorithm (Figure 2-26). New branches grow toward the brightest direction. The model also simulates hormonal control of plant growth by dormancy break. After meeting a given set of preconditions, especially by low inhibition, a dormant but could germinate or die according to its light environment. This model was developed for generic plants and simulates growth only qualitatively.

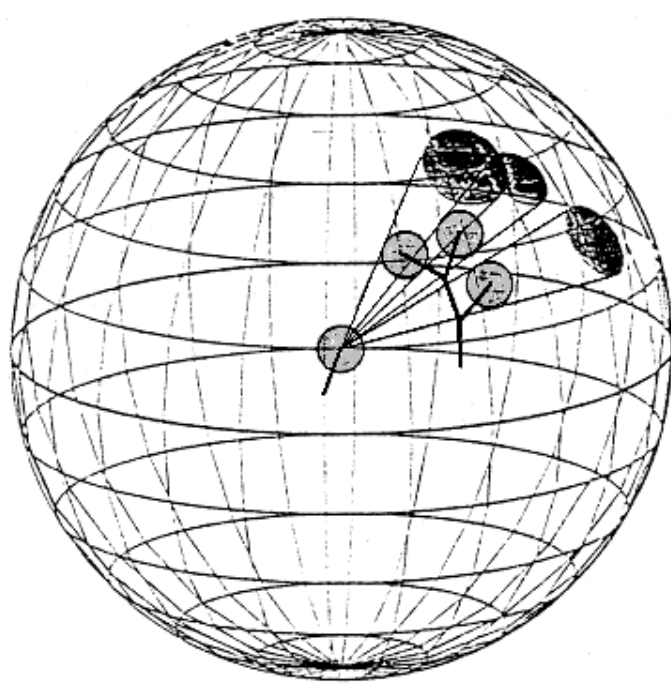

Figure 2-26: Celestial sphere model of CHIBA ET AL. (1994). Incoming radiation is calculated using the projected shadow or the leaf clusters on the sphere. From the lower hemisphere comes only diffuse radiation.
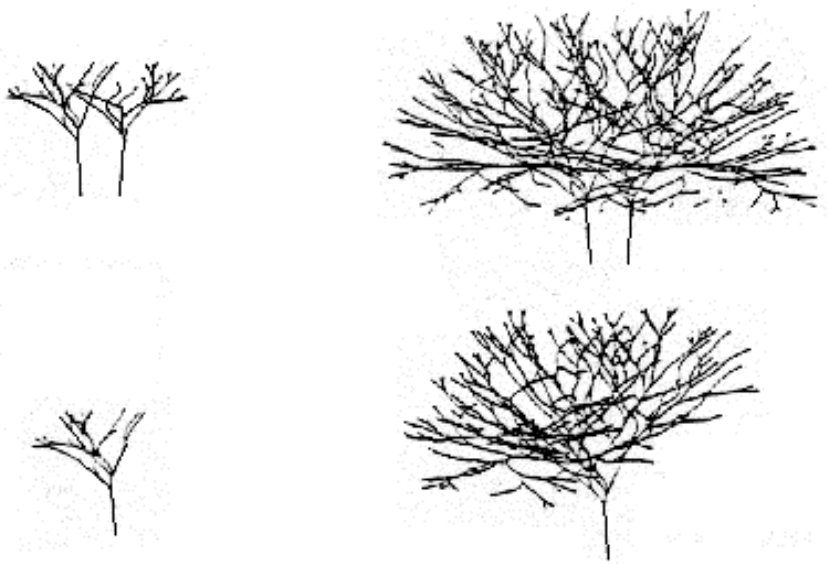

Figure 2-27: Examples of simulation results from CHIBA ET AL. (1994). Above, simulation of normal growth. Below simulation of the crown restoration after isolating one of the trees. 
The plant growth model MADEIRA (LIST AND KÜPPERS, 1998; LIST ET AL., 1994) describes the plant structure with a modular approach as typical for object oriented models. Light interception is calculated using a voxel discretisation. Leaf area is distributed among the voxels to create light absorbing filters. The volume of a voxel can be modified as needed. The authors propose a voxel side length of $10 \mathrm{~cm}$ to be adequate for most cases. Incoming radiation is not calculated as exactly as in the models presented above. The light source is considered to be homogeneous on top of the area of the plant population, because growth is integrated over a whole season. Position and orientation of individual leaves are used to calculate their exposition, and thus the capacity of exploit short phases of high light intensity, as by sun flecks.

After light interception, assimilation is calculated and assimilate pools are created. From these pools, the amount needed to support growth and maintenance is deduced and the rest is passed to the next lower segment. The flow of assimilates occurs from leaves towards the roots. There are critical maintenance values that must be paid or the respective shoots die. The branching patterns are sensible to the assimilate distribution. The greater the amount of assimilates available, the longer are the created new shoots. The model tries to calculate an optimal development of new created shoots in relation to the parent shoots. MADEIRA can be parameterised for different species with different branching patterns and was tested on different species as Acer campestre and Prunus spinosa. Figure 2-28 shows the voxelspace surrounding a canopy and the distribution of leaves within it.

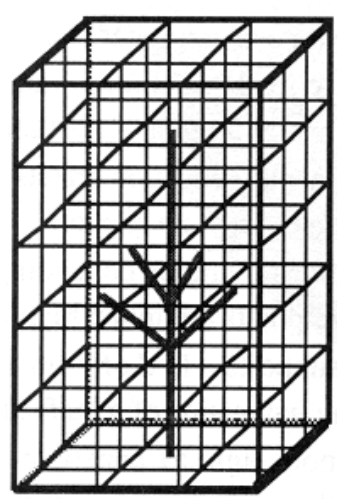

a

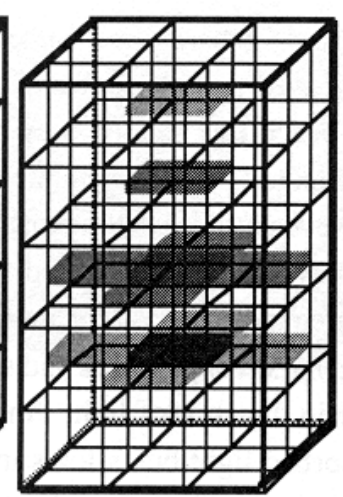

b

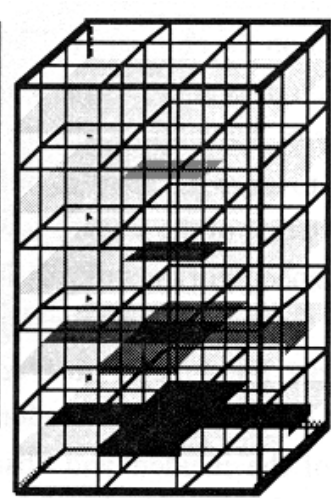

Figure 2-28: Representation of the voxelspace surrounding the canopy of a plant with MADEIRA. a) The plant in the voxelspace. b) The distribution of the leaf area among the voxels. b) Light reduction (after LIST AND KÜPPERS, 1998). 
TAKENAKA (1994) developed a light reaction model which uses a system similar to the model of Chiba et al. (1994). Incoming radiation is calculated using a hemisphere which is divided into 20 segments with different brightness. The architecture of trees is represented using "branch units" as part of a modular structure. The central stem grows vertically. There are three lateral branches produced each year separated $135^{\circ}$ from each other and inclined $60^{\circ}$ from the central axis. New units on lateral axes are located on the same plane. Each branch can produce up to three new branches with a branching angle of respectively $5^{\circ},-50^{\circ}$ and $60^{\circ}$ (or with reversed angles). The leaves are clustered at the distal end of new branch units. The clusters are represented with spheres whose size is proportional to the leaf area and to the light permeability of the cluster. Assimilation is calculated in function of illumination. The leaf mass is subtracted from the amount of produced assimilates. The rest is the net gain of organic matter $(\mathrm{P})$ and is used for branch growth in the following year. Takenaka set the boundary values Smax, Smin (maximal and minimal size of new branch units) and Nmax (maximum number of branch units that can be created, normally 3 ). The mass of a new branch is then calculated as below:

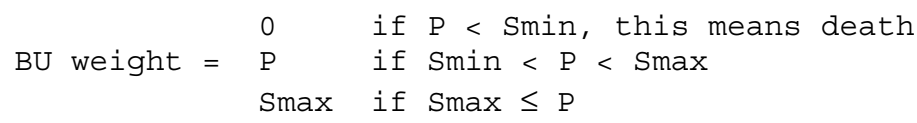

Branches are produced as long as there are enough assimilates $(\mathrm{P}>0)$ or until Nmax is reached. The length of the new branch unit and its leaf area are proportional to the weight calculated. There exists a factor $s$ Y 1 that describes how much the surface of the leaf sphere is filled with leaves. The probability that a light ray hits a leaf in the cluster is $1-1 / s^{2}$. If $s=1$ the leaf area is equal to the surface of the sphere and the probability of being hit is 1 . The transmittance $t$ of a single leaf is given as a constant, the transmittance of the cluster is given by the formula $t_{l c}=\left(1-(1-\mathrm{t}) / \mathrm{s}^{2}\right)^{2}$, and the light absorption by $1-t_{l c}$. Light absorption is then calculated for all the 20 sectors of the light hemisphere. For the light transmission only the clusters are considered to have shadows, the segments representing branch units are ignored. This model could be expanded to simulate more realistic branching structures or even "real" plant species. KURTH (1999) created an L-system that emulates this model with some minor differences. Figure 2-29 shows a simulation example of a solitary tree. 


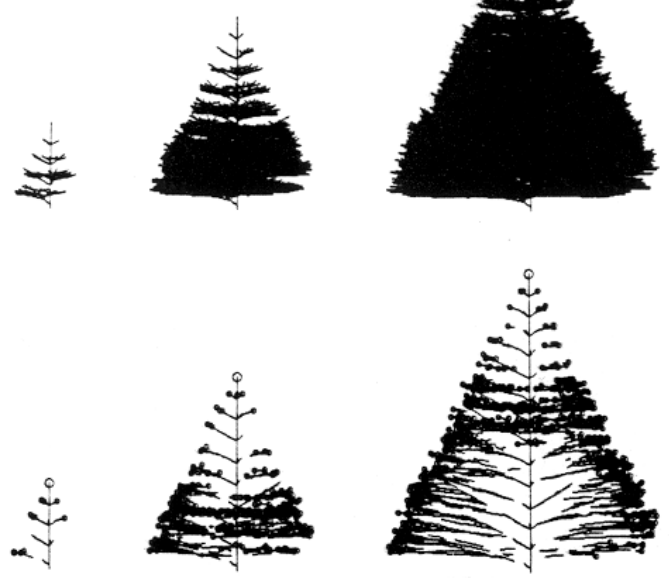

Figure 2-29: Simulation of a solitary tree with the light response model of TAKENAKA (1994) (above) and cross-sectional view (below).

The later version of the model of KELLOMÄKI AND STRANDMAN (1995) for Scots pine (Pinus sylvestris) was originally only a structure model that was further developed. The authors describe a part of the length growth of shoots as a function of light intercepted. "The intercepted light is converted to the respective length..." In this process, diameter and wood density are parameters of the light conversion function. Further growth is calculated using the mechanistic approach of McMahon, which describes diameter growth as the reaction to gravity-induced forces at each branching point. Figure 2-30 shows the calculated light interception.
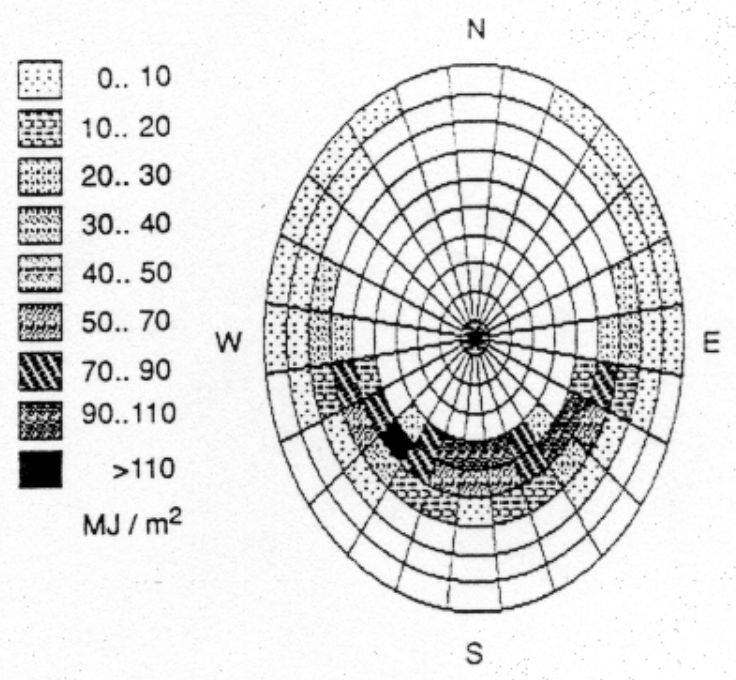

Figure 2-30: Calculated intercepted light with a hemispherical model (after KELLOMÄKI AND STRANDMAN, 1995).

The model uses a hemispherical interception model with 240 segments. For comparison, the model MIR from Dauzat (section 2.1.4.1) uses a hemisphere with "only" 46 sectors. The 
model uses the given tree structure to calculate self shadowing. Figure 2-31 shows a tree simulated with this model.

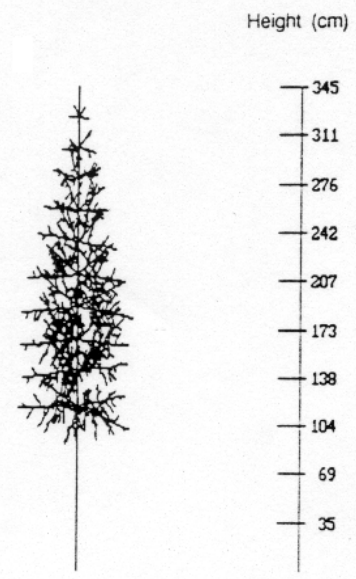

Figure 2-31: Image of a simulated tree by Kellomäki and Strandman.

The workgroup of Risto Sievänen at the METLA, Finland, developed a C-allocation model for single trees called LIGNUM (PERTTUNEN ET AL., 1996, 1998; SiEVÄNEN ET AL., 1995). The plant structure is built using three main elements: tree segment, branching point and bud. Tree segments are divided into heartwood, sapwood and foliage which are modelled as cylinders. The root system forms a single compartment that functions as an assimilate sink. The length of a tree segment is calculated according to its branching order and the local light conditions. The number of new segments generated is a function of the vitality of the parent segment which is proportional to its foliage mass. Secondary diameter growth is calculated according to the pipe model (compare 2.1.7).

The light model used is, like the models of CHIBA ET AL. (1994), SHINOZAKI (1994) and DAUZAT $(1993,1994)$, based on a ray tracing method using a sky hemisphere divided into sectors. For light interception, LIGNUM uses a voxel space like the model MADEIRA (LIST ET AL., 1994). For the C-allocation, the net assimilation of the whole tree is calculated and a central pool is created. From this pool resources are then distributed among the different elements. The amount of resources needed to produce new segments, new roots and secondary growth depends upon an unknown factor $\lambda$ for length growth (intensity factor), which is approximated with an iteration method. LIGNUM is a good example for object oriented modelling (compare section 3.3) and is suitable for interconnection with other models. KURTH (1999) developed a program shell called GROCOM which combines GROGRA, a LIGNUM re-implementation and different radiation models to reproduce the 
results of the original LIGNUM model. Figure 2-32 shows some examples of simulations with LIGNUM.

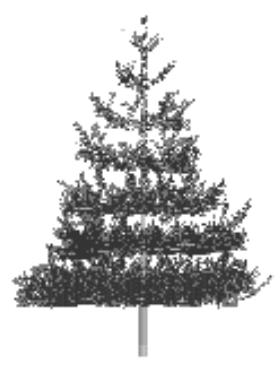

a

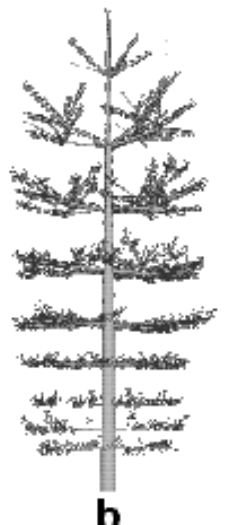

b

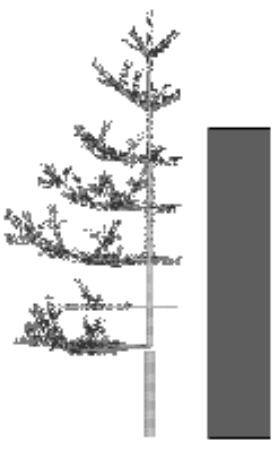

c

Figure 2-32: Simulation of growth in dependence on light. a) Tree adapted to grow in shade. b) Tree with increased light sensitivity. c) Effect of partial shadowing through an obstacle. (After www.metla.fi, 2002).

The model SIMWAL (SIMulated WALnut) (BALANDIER ET AL., 2000) simulates the threedimensional structure of young walnut trees (Juglans regia) and its dynamics. A main focus of SIMWAL is the simulation of the effect of silvicultural practices, especially pruning. The architecture and topological structure of the tree are described using the AMAP definition of growth units (see section 2.2.4.3). The model includes some sub-models described in section 2.1. to calculate light interception (radiation extinction after Beer's law) and photosynthesis (model of FARQUHAR ET AL., 1980). C-allocation is calculated using a proportional approach, i.e. the amount of resources that are allocated to a sink is proportional to its demand (LACOINTE, 2000), with some extensions. Due to an object oriented modelling approach used to describe tree organs, SIMWAL has great potential for being expanded and combined with other models. However, the basic models used have been parameterised only for Juglans regia making it difficult to adapt to other trees. A similar $\mathrm{C}$-allocation model to simulate the effect of pruning in $\mathrm{N}_{2}$-fixing trees (namely Gliricidia sepium) was developed by BERNINGER ET AL. (2000). This models includes besides C-allocation also $\mathrm{N}$-allocation for describing the process of $\mathrm{N}_{2}$-fixing.

BosC (2000) developed an object oriented ecophysiological model called EMILION (Ecophysiological Modelling Integrating Linked OrgaNs) that has been parameterised for Pinus pinaster. Using stand and tree structure and meteorological data, EMILION calculates 
$\mathrm{C}$-allocation and the distribution of water fluxes. The model works on organ level and takes into account the relationships between different organs. Allocation and water fluxes are calculated in short time steps of approximately 30 minutes. Light interception is calculated according to the geometry and orientation of shoots. Photosynthesis is calculated with the model of FARQUHAR ET AL. (1980). Stomatal conductance is calculated using a multiplicative approach (JARVIS, 1976). Transpiration is calculated as a function of stomatal conductance, leaf area and water vapour deficit. Leaf temperature is assumed to be equal to that of air. Water flow is equal to the transpiration of a shoot plus the flow of supported shoots. The model needs to know the initial and final dimensions of an organ to simulate its evolution between these two states according to its phenology. Branch mortality was simulated through the carbon balance, i.e. death occurs when the carbon balance becomes negative.

JALLAS ET AL. (2000) developed a simulation model for cotton plants (Gossypium spc.) called COTONS that includes a plant and a soil model. The model needs data about soil hydrology, stand characteristics and meteorological data. COTONS uses many sub-model to represent the architecture and the morphology of cotton plants and simulates growth based on a daily allocation model. This model is based on an older cotton model called GOSSYM and is thus limited to only one species. The sub-models used seem highly specialised and are not described clearly. However, the general approach of modular sub-models can be used to develop more generic models.

A similar situation is given in the model ALMIS (ESCHENBACH, 2000), which simulates light acclimation of black alder (Alnus glutinosa L. (Gaertn.)) trees. ALMIS is an object oriented model with the objects Internodes, Leaves, Meristems, Roots and Roottips as structure objects (compare section 3.3). The environment is also handled with own objects for air and soil segments. The environment is represented by a voxel space with $8 \times 8 \times 12$ (768) voxels. ALMIS simulates growth based on carbon fluxes. Each structure object possesses pools for assimilates, "starch" and structural carbon. Starch represents as storage pool, and structural carbon is fixed in the growth process. The model is able to handle different types of trees: sun type with only sun leaves, shade type with only shade leaves and adaptive type with sun and shade leaves distributed according to the light environment. The time discretisation is regulated by the meteorological data. The light model used is a simple model based on Lambert-Beer's Law and comparable to the model used in MADEIRA (LIST AND KÜPPERS, 
1998). The model was used on isolated and competing trees to describe the adaptation capacities of the different types of leaves. Figure 2-33 shows simulation results for isolated trees. As many other models presented here, the main flaw of ALMIS is its restriction to only one tree species. The principal approaches, however, are based on general botanical observations that are valid for other species, too. With a generic parameterisation procedure, the model could be applied to other species.

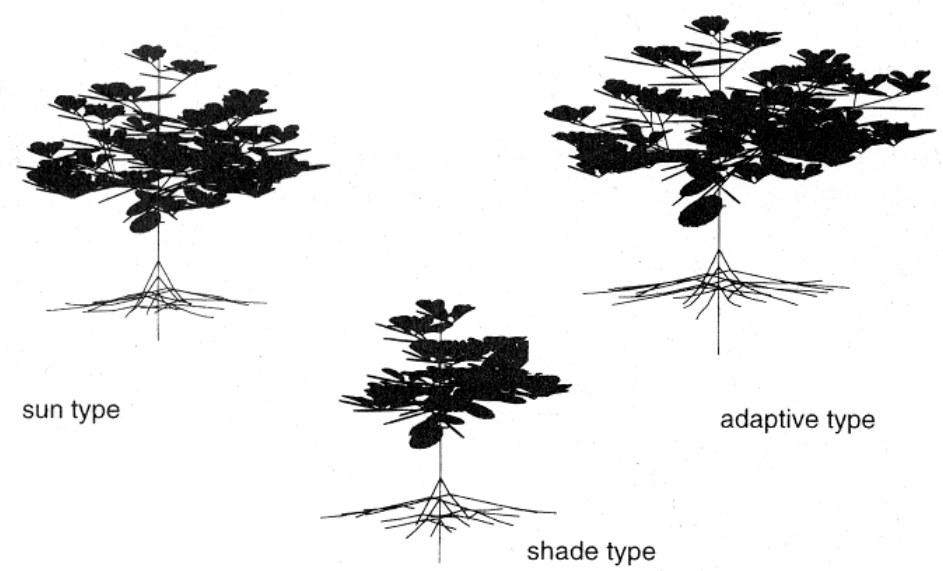

Figure 2-33: Three isolated trees modelled with ALMIS after 150 time steps. The sun type has only sun leaves, the shade type only shade leaves and the adaptive type sun and shade leaves distributed according to the light environment.

PAGÈS (2000) describes some methods for functional-structural modelling of root systems by including organ interactions in architecture models. The approaches described here are similar to those used in the "above-soil" models presented above. While most models handle root systems as single compartment representing a carbon sink, Pagès proposes to handle the above ground parts of the plant as a single compartment acting as a source of carbohydrates. Clausnitzer AND Hopmans (1994) combined root growth with soil water flow. Models combining both above-ground and below-ground models are not yet fully realised. The NAPAP project attempted to combine many different models, including root systems and mycorrhizae (KIESTER ET AL., 1990). There are also more specialised models that could be combined with some of the models presented here as for example the gas exchange models of FALGE ET AL. (1996, 1997). The level of structural information is also variable. Models simulating whole stands tend to simplify tree structures (PFrEUndT, 1988; PFREUNDT AND SlOBODA, 1996; PreTZSCH, 1990a, 1990b, 1992a, 1992b). 


\section{MATERIAL AND METHODS}

\subsection{Validation material}

For the validation of the NEXUS software plant material and meteorological data were collected from two sampling plots. Also data provided by Jean Dauzat and Bruno Rapidel for Coffea arabica and meteorological data for Costa Rica were used.

\subsubsection{Sampling plots}

\subsubsection{Costa Rica}

The data provided by Jean Dauzat were measured in a private coffee (Coffea arabica) stand (RAPIDEL, 1995; DAUZAT AND RAPIDEL, 1998; RAPIDEL ET AL., 1999) of 12 ha near Turrialba, Costa Rica $\left(9^{\circ} 22^{\prime} \mathrm{N}, 83^{\circ} 22^{\prime} \mathrm{W}\right)$ at an altitude of $600 \mathrm{~m}$ above sea level. The coffee plants grow in homogeneously planted rows mixed under Erythrina poeppigiana (Walpers) O.F. Cook. The stand was planted 1983, the coffee plants were measured 1994 at an age of 11 years.

The stand grows on a young volcanic soil with typical aluminium-silicates (allophanes) and high drainage capacity. Climate in the region is hot and humid, with a mean precipitation of $2600 \mathrm{~mm} /$ year and a mean temperature of ca. $22{ }^{\circ} \mathrm{C}$.

A 15 meter high pole is situated in the centre of the stand for measuring meteorological data. The data used by RAPIDEL (1995) was measured with instruments installed on this pole at a height of 5 meters. 


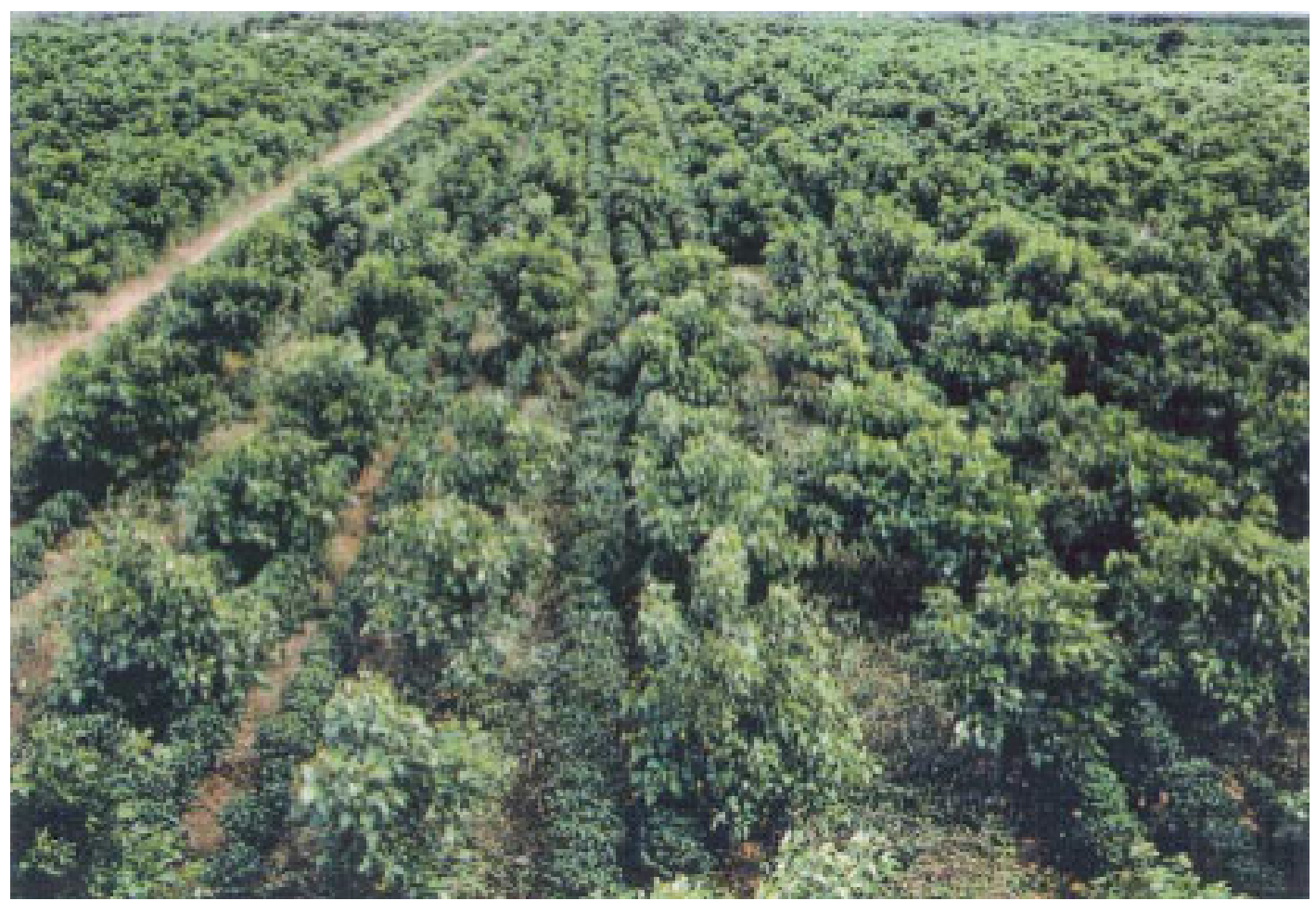

Figure 3-1: Picture of the coffee stand in Turrialba, Costa Rica, made from the top of a $15 \mathrm{~m}$ high measurement tower situated in the centre of the stand (RAPIDEL, 1995).

\subsubsection{Germany}

The sampling plots are located $2 \mathrm{~km}$ west from Unterlüß $\left(52^{\circ} 45^{\prime} \mathrm{N}, 10^{\circ} 30^{\prime} \mathrm{E}\right)$ in the Lüneburger Heide (Germany) at an altitude of 115 meters above sea level. The plots were established for different projects for the research of forest ecosystem dynamics that have been carried out since 1986 in numerous diploma and doctoral theses at the Georg-August University of Göttingen (CONERS, 2001).

The plots are situated in the south-west part of the north-west German lowlands, corresponding to the transient area from the moraines of the Lüßberge into the Sprakensehler outwash plains. Through the deposition processes in the Saale ice age and the Holocene the main soil type is a low nutrient sand soil sustaining an acidic podsol brown earth.

The first plot is a fenced area with mixed vegetation, where Quercus spc. rejuvenation grows below an old stand of Pinus sylvestris. Due to the increased light incidence through the open canopy, the herbal vegetation is relatively abundant, with exemplars of Avenella flexuosa, Carex pilulifera and some lichens and mosses. There are also single specimens of Fagus 
silvatica, Quercus robur, Quercus hybrids, Picea abies and other species. The second plot is situated near the first plot and consists of a closed mixed stand of 100 years old Fagus silvatica and 190 years old Quercus petraea with a mean tree height of $28 \mathrm{~m}$. A $36 \mathrm{~m}$ high tower has been installed within the plot to allow measurements in and above the canopy, and it provided the meteorological data used to validate the software.
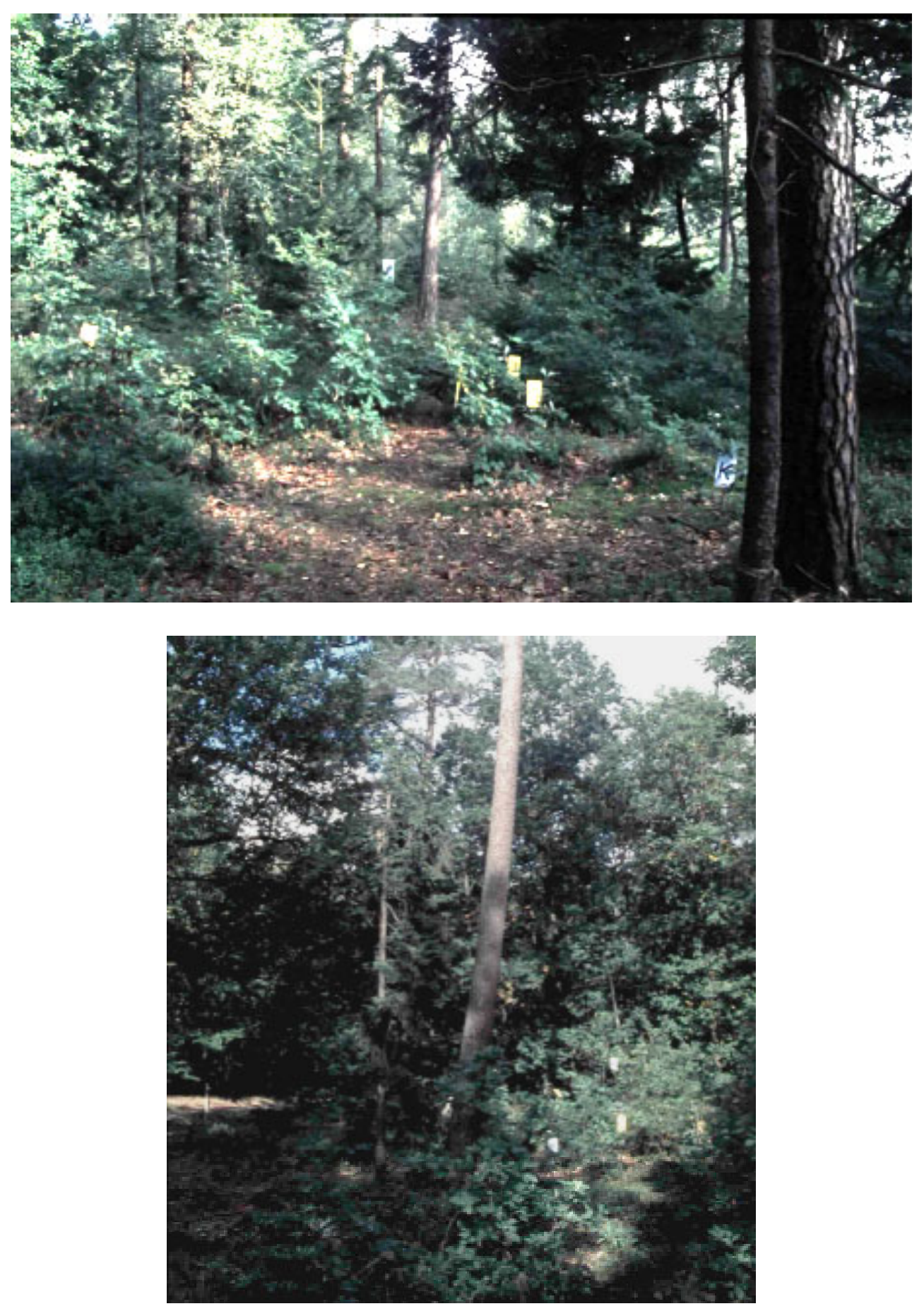

Figure 3-2: Mixed stand in Unterlüß, Germany. Old growth stand, consisting mainly of Pinus sylvestris with mixed rejuvenation. View from west (above) and north (below). 


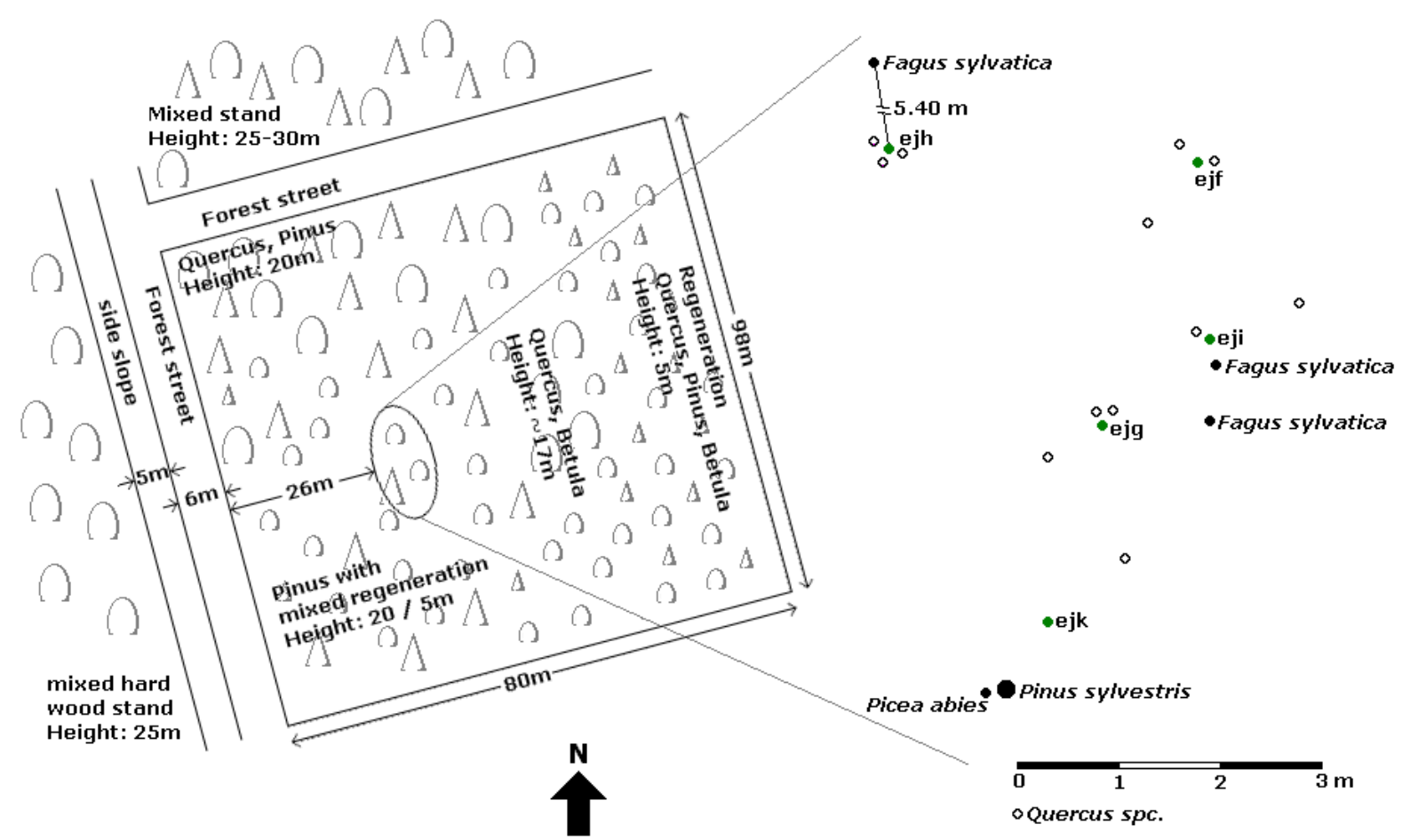

Figure 3-3: Scheme of the sampling stand in Unterlüß and of some of the collected trees. The stand is fenced and very inhomogeneous. In the north, there are mostly old trees (Quercus and Pinus) with almost no rejuvenation. At the east, the old trees have been cut. The rest consists of some old trees, especially Pinus sylvestris, above a rich mixed rejuvenation, predominantly Quercus spc. Besides the five sampling trees from the middle represented here, two other exemplars were collected at the eastern part of the stand. Besides the sampling trees, significant neighbours and their respective species have been represented.

The region of the Southern Lüneburger Heide has a temperate-cool sub-oceanic climate. According to data from the station of the German Meteorological Service (Deutscher Wetterdienst, DWD) in Unterlüß, the mean air temperature is $8.1{ }^{\circ} \mathrm{C}$, and the annual precipitation $801 \mathrm{~mm}$.

\subsubsection{Meteorological data}

There are two sets of meteorological data used to validate the software. The first set has been provided by Jean Dauzat. The meteorological data was measured every 5 seconds, saving the mean values every 30 minutes over a long period of time. For the validation, only data measured at an exemplary sunny day (April, 13 $\left.{ }^{\text {th }} 1994\right)$ and at an exemplary cloudy day (April, 27 $7^{\text {th }}$ 1994) were used (Figure 3-4). The second set of meteorological data comes from the observation tower located in the sampling plot described under 3.1.1. The data were measured from April, $12^{\text {th }}$ at 9:45 a.m. till April $13^{\text {th }}$ at $6: 00$ p.m. every 30 minutes. The set contains global radiation $\left[\mathrm{W} / \mathrm{m}^{2}\right]$, air temperature $\left[{ }^{\circ} \mathrm{C}\right]$ and relative air humidity $[\%]$. 


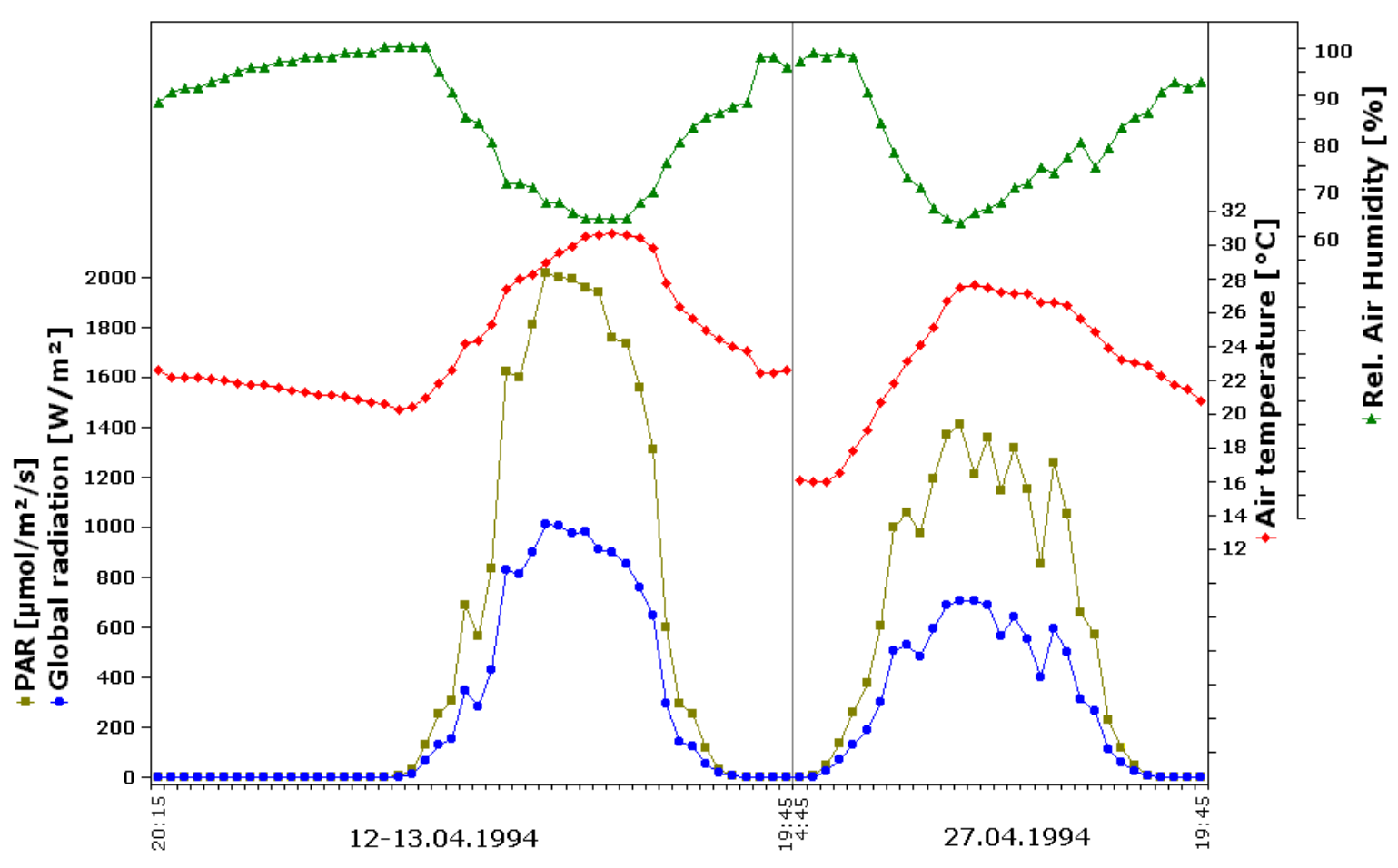

Time

Figure 3-4: Meteorological data from Turrialba, Costa Rica, measured at a sunny day (april $13^{\text {th }}$ ), and at a coudy day (april, $27^{\text {th }}$ ). The data for the night from april $26^{\text {th }}$ to $27^{\text {th }}$ has been ommited (Data provided by Jean Dauzat, CIRAD, France).

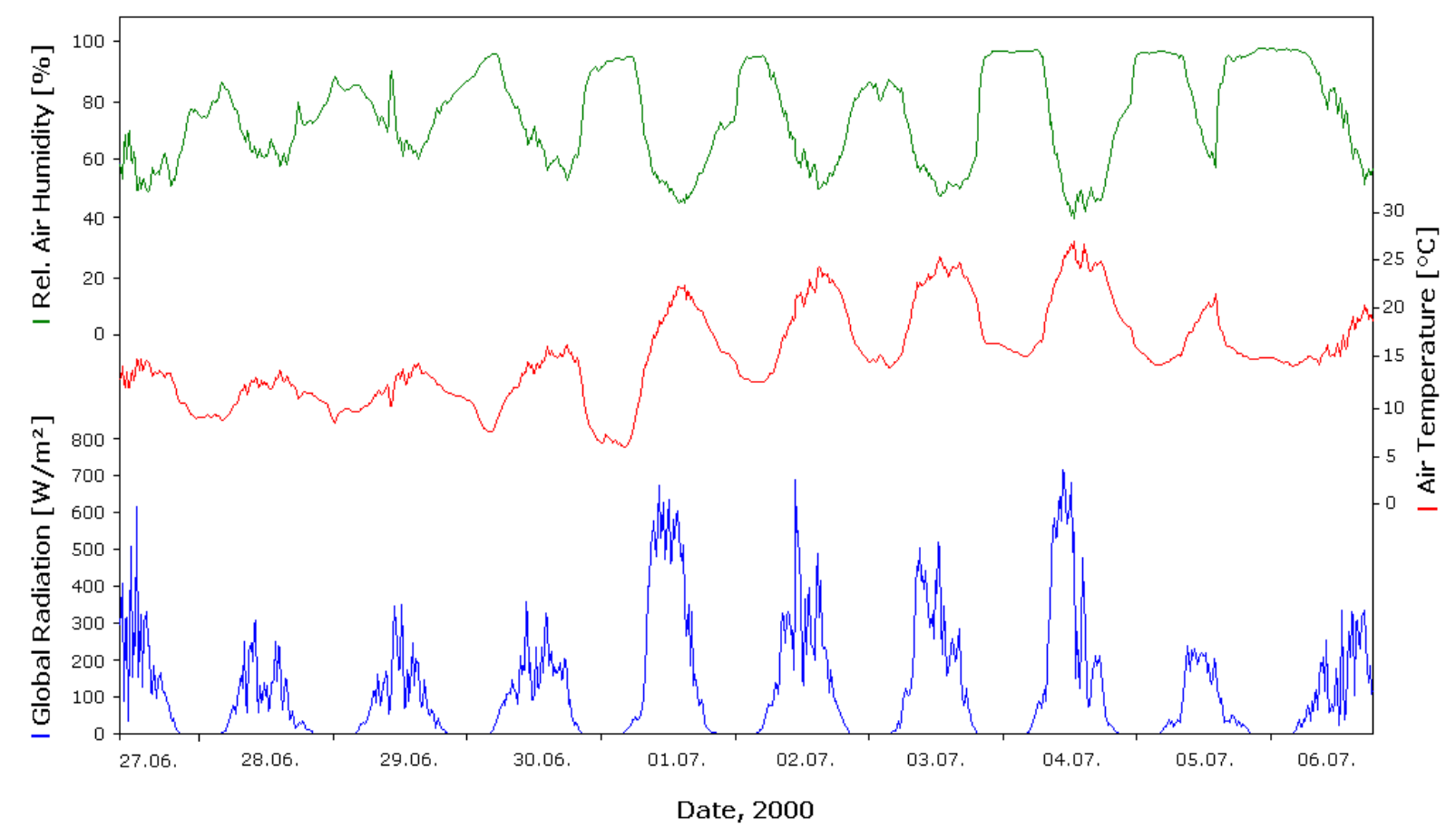

Figure 3-5: Global radiation, air temperature and relative humidity values measured from June $27^{\text {th }}$ till July $6^{\text {th }}$ in Unterlüß, Germany. All values were measured at a height of $36 \mathrm{~m}$ every 30 minutes.

\subsubsection{Plant material}


Jean Dauzat provided the digitised data of a Coffea arabica plant from Turrialba, Costa Rica. The data are coded in AMAP format (version 2.2) and were used for comparison with previous results from the original software for the model MIR/MuSc and HYDRO in order to validate the results from NEXUS. For details about data collection see RAPIDEL, 1995.

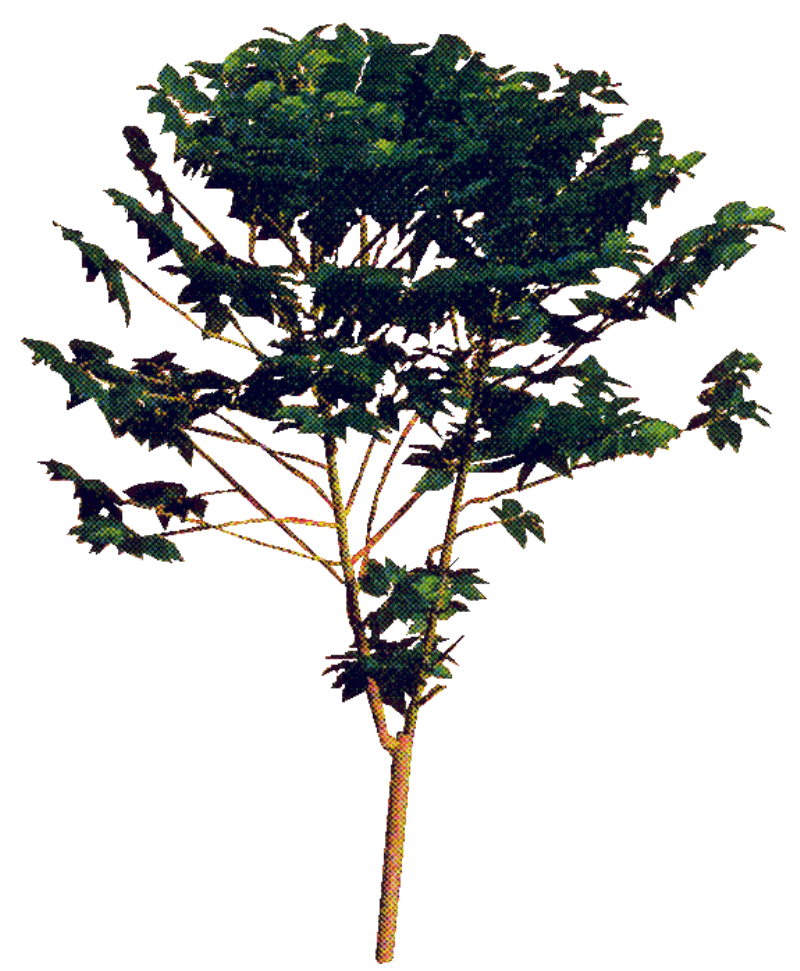

Figure 3-6: Representation of a Coffee plant from Costa Rica generated with the tool "Landmaker" from the AMAP software.

From the sampling plot in Unterlüß described in section 3.1.1 seven exemplars of oak (Quercus robur and Quercus petraea) were selected under consideration of light, growth density and age, to gain a vast range of different growth conditions and stages. The trees are named: ejc, ejd, ejf, ejg, ejh, eji and ejk, where $e$ means "Eiche" (German for oak), $j$ means "jung" (German for young) and the last letter is an identifier. From originally eleven preselected trees, only seven were selected for this investigation. Before sampling, a series of water flow measurements were made under different light conditions, especially to measure the reaction and the reaction time of water flow to sudden shadowing and exposition to light (SCHUlTE AND ČERMÁK, personal communication). After sampling, for each branch the following information was collected: 
- branch length, in mm

- branch diameter, in $\mathrm{mm}$, accurate to $0.1 \mathrm{~mm}$

- position of side branches measured from the base, in $\mathrm{mm}$

- branching angle, accurate to $5^{\circ}$

- number of leaves

- other relevant information, like dead or broken terminal buds, reiteration events, etc.

The data for each tree were graphically mapped and then compiled in dtd-format (descriptive tree data) for the representation with GROGRA (KURTH, 1994). Additionally, leaf area and dry mass of leaf and branches after 48 hours in a drying furnace at $105{ }^{\circ} \mathrm{C}$ were measured. Table 3-1 shows an example of a dtd-file and Figure 3-7 the corresponding map and its representation with GROGRA.

\begin{tabular}{|c|c|c|c|c|c|c|c|c|}
\hline 1 & L52 & \#\# & 01 & R3 & W90 & $\mathrm{D} 1.6$ & $\mathrm{C} 2$ & \\
\hline 2 & L60 & \#1 & $\mathrm{V}$ & R1 & W2 0 & $\mathrm{D} 1.6$ & $\mathrm{C} 2$ & \\
\hline 3 & L50 & \#2 & $\mathrm{V}$ & & & $\mathrm{D} 1.2$ & $\mathrm{C} 2$ & \\
\hline 4 & L4 3 & \#3 & $\mathrm{V}$ & & & $\mathrm{D} 1.2$ & $\mathrm{C} 10$ & $\{N\}$ \\
\hline 5 & L29 & \#2 & A3 2 & - & W8 0 & Do. 8 & $\mathrm{C} 2$ & \\
\hline 6 & L26 & \#5 & $\mathrm{V}$ & & & Do. 5 & $\mathrm{C} 10$ & $\{N\}$ \\
\hline 7 & L50 & \#2 & A52 & - & W65 & $\mathrm{D} 1.4$ & $\mathrm{C} 2$ & \\
\hline 8 & L36 & \#7 & $\mathrm{V}$ & & & $\mathrm{D} 1.0$ & C6 & $\{\mathrm{ON}\}$ \\
\hline 9 & L28 & $\# 7$ & A4 6 & - & W50 & D1.0 & $\mathrm{C} 10$ & $\{\mathrm{~N}\}$ \\
\hline 10 & L22 & \#7 & A44 & + & W65 & Do. 8 & C10 & $\{\mathrm{N}\}$ \\
\hline 11 & L4 2 & \#3 & A4 7 & - & W70 & Do. 8 & $\mathrm{C} 6$ & $\{\mathrm{ON}\}$ \\
\hline 12 & L41 & \#3 & A 47 & + & W70 & $\mathrm{D} 1.1$ & C10 & $\{\mathrm{N}\}$ \\
\hline 13 & L50 & \#2 & A54 & + & W60 & $\mathrm{D} 1.1$ & $\mathrm{C} 2$ & \\
\hline 14 & L43 & \#13 & $\mathrm{V}$ & & & $\mathrm{D} 1.0$ & C10 & $\{N\}$ \\
\hline 15 & L2 8 & \#13 & A4 5 & - & W65 & Do. 9 & $\mathrm{C} 10$ & $\{\mathrm{~N}\}$ \\
\hline 16 & L35 & \#13 & A4 6 & + & W60 & $\mathrm{D} 1.0$ & $\mathrm{C} 10$ & $\{\mathrm{~N}\}$ \\
\hline
\end{tabular}

Table 3-1: Example for the description of a plant structure using dtd (digitised tree data) format. The columns mean: identifier, length $[\mathrm{mm}]$, mother segment (\#\# is the base segment), location on the mother branch [mm] (V means the same axis), branching direction (+ right, - left), branching angle, diameter $[\mathrm{mm}]$, colour and possible attributes.

The close environment of each tree was mapped for the reconstruction of the scenes with the AMAP software tool Landscape. Relevant neighbours were mapped using a polar coordinates system centred on each sampling tree. Distance was measured in centimetres, accurate to $0.5 \mathrm{~cm}$, and angles were measured in reference to the north in degrees. Relevant neighbours are those trees that could affect the direct environment of the sampling tree, including old trees which modify wind and light environment. Figure 3-3 represents a scheme 
of the sampling plot and of the distribution of some of the sampling trees together with their relevant neighbours. The data of the sampling trees were converted into the AMAP linetree format using the interface provided by GROGRA in order to make it accessible for the Landmaker software.
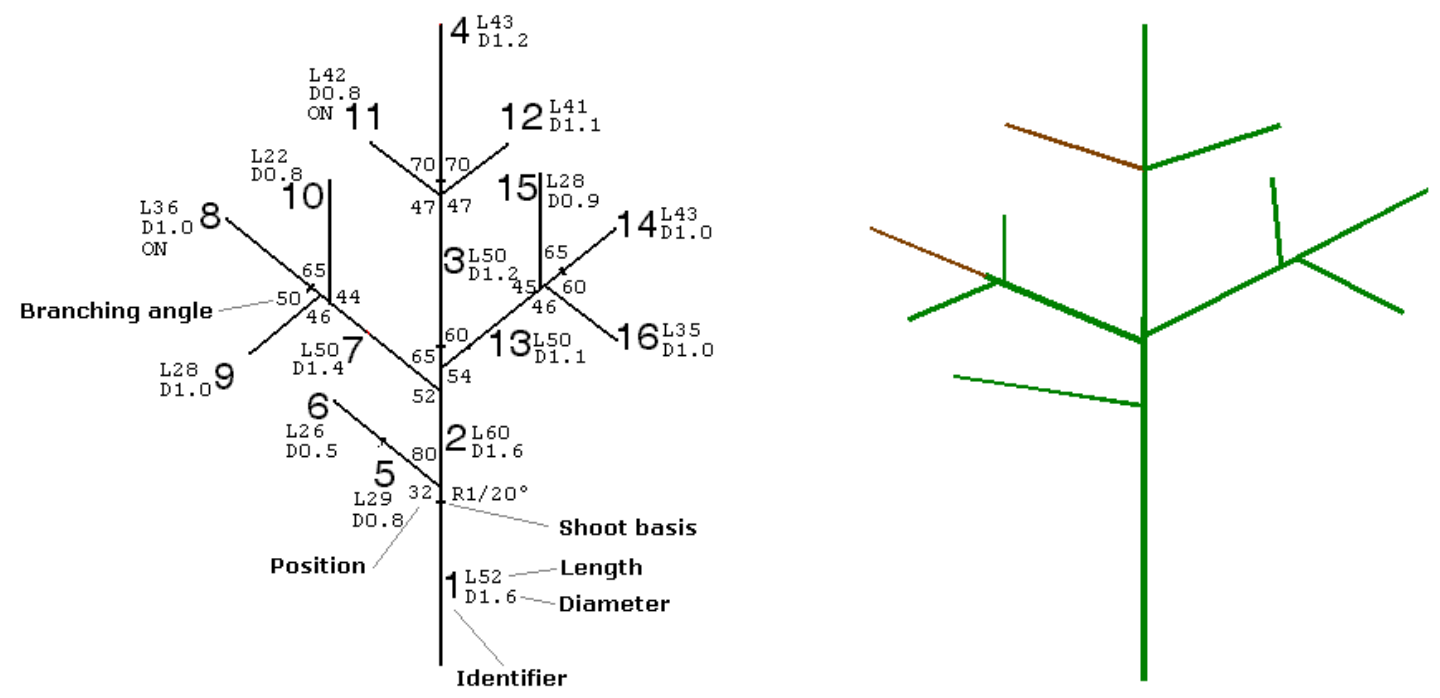

Figure 3-7: Example of a mapped structure and its representation with GROGRA.
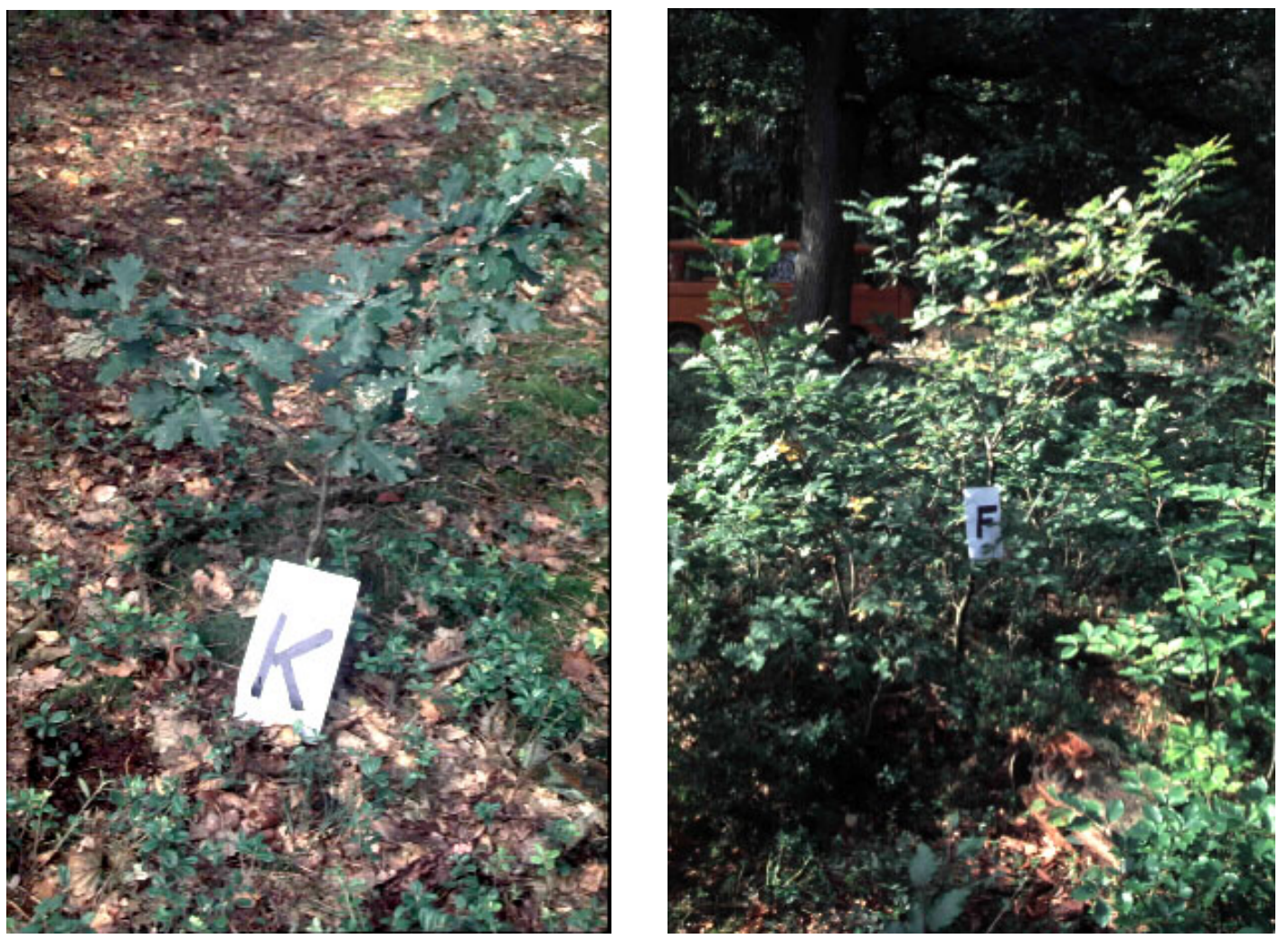

Figure 3-8: Sampling trees ejk (left) and ejf (right). Tree ejk grows below a pine tree with a $d b h$ of $30 \mathrm{~cm}$ and a height of ca. $25 \mathrm{~m}$. This tree was measured in situ. Tree ejf grows within a rejuvenation group and was measured destructively after sampling. 

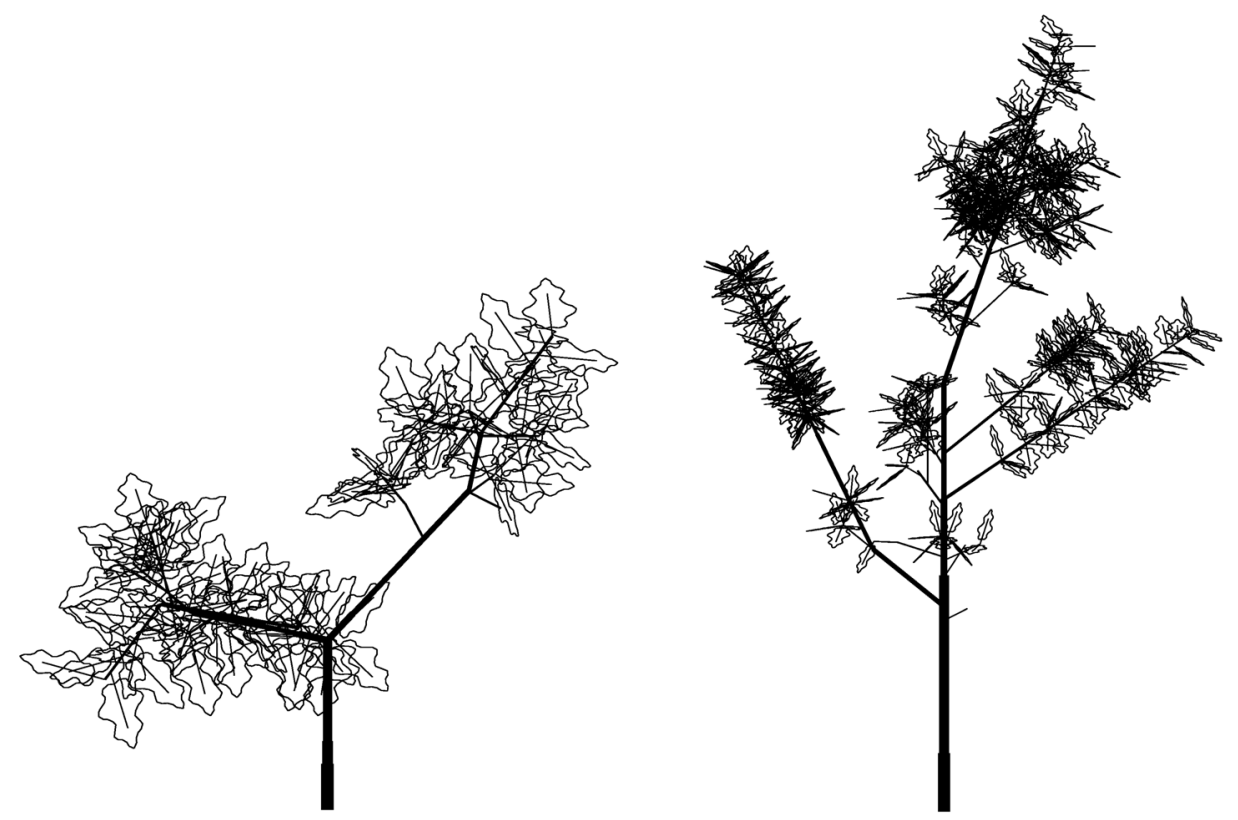

Figure 3-9: Representation of the trees ejk (left) and ejf (right) with GROGRA.
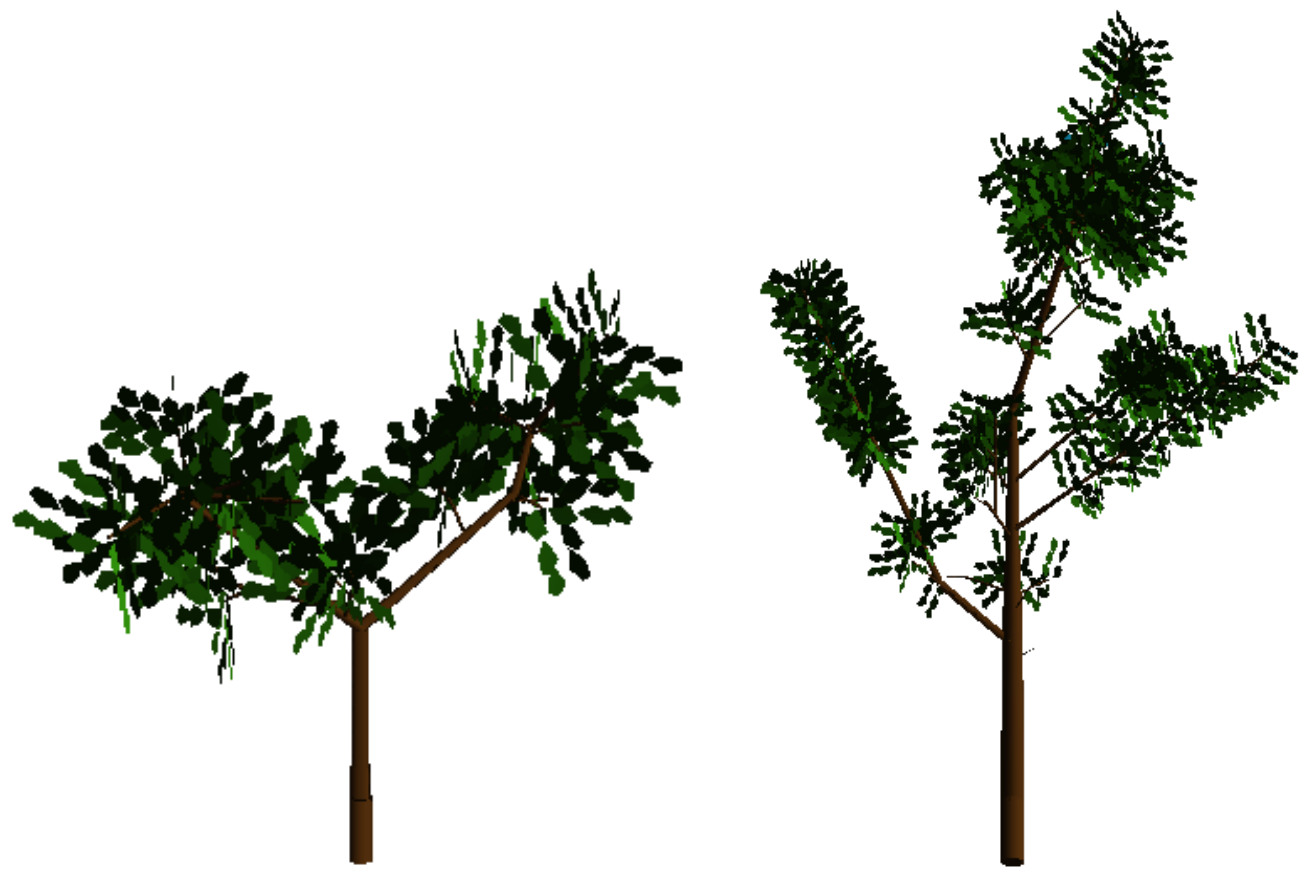

Figure 3-10: Representation of the trees ejk (left) and ejf (right) with AMAP.

Sap flow was measured for the trees ejk, ejh, eji, ejf and ejg parallel to the measurement of meteorological data using a "6-channel-sap-flow-meter" type T693.2 (EMS, Brno) with the stem heat balance method from ČERMÁK ET AL. $(1973,1976)$. Data for tree ejk were collected only till July $3^{\text {rd }} 2000$. In tree eji there were two measurement points, eji low for standard 
collar measurement and eji high for a single branch on the crown. For the comparison of results only eji low was used. Figure 3-11shows the measured sap flow data.
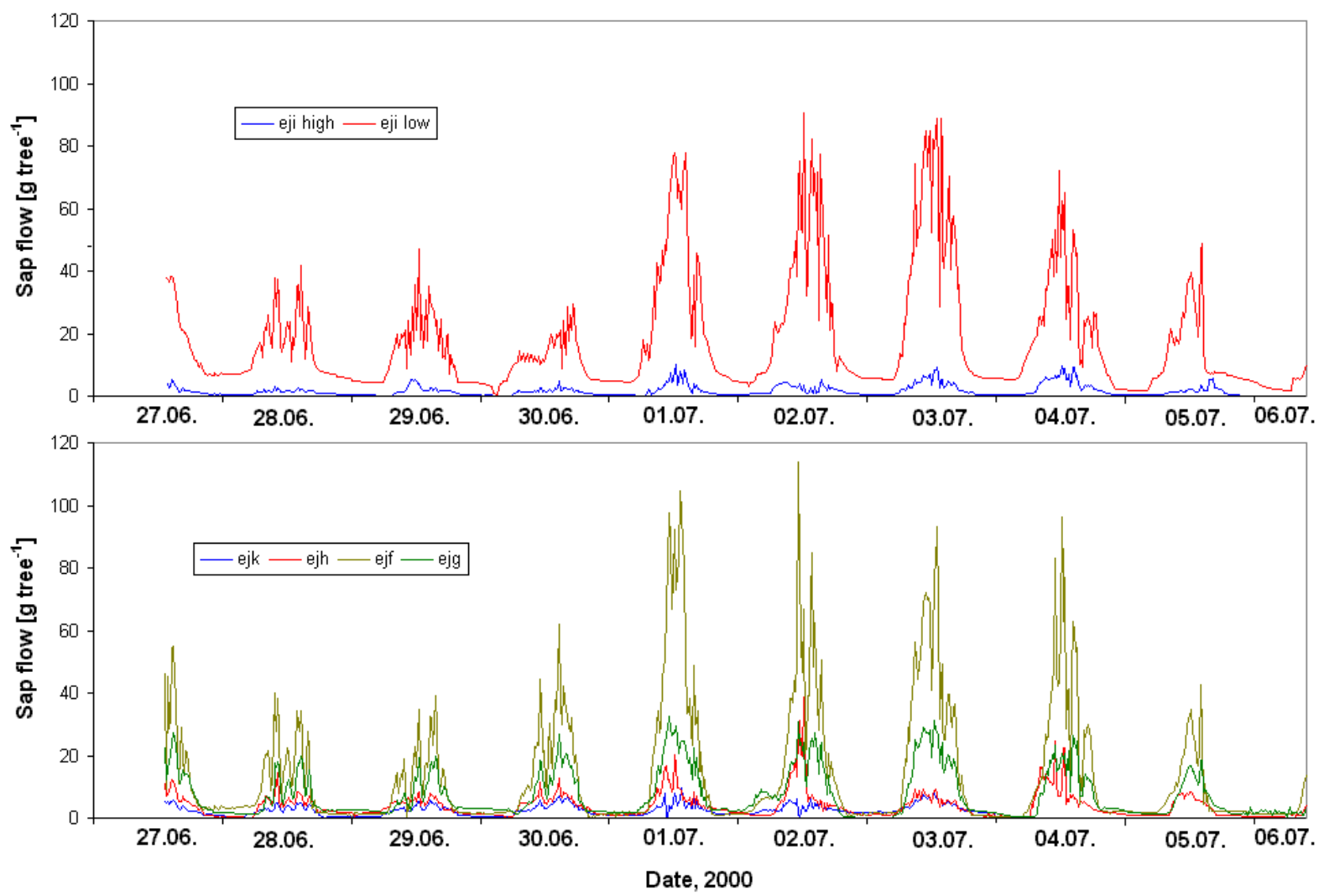

Figure 3-11: Measured sap flow for the tree ejk, ejh, ejf, ejg (below) and eji (above). On tree eji two separate sap flows were measured, once on the collar (eji low) and once on a branch in the upper part of the crown (eji high).

\subsection{Data formats}

To reach the desired level of effectiveness it is important first to achieve a general standard for data files. The first modules to be integrated each offer a set of data files that are strongly specific and show normally little flexibility. These modules and their corresponding file formats are listed in Table 3-2.

Using files is a common method for data transfer. But as shown in Table 3-2, already with the few modules presented here the quantity of different files provided is vast. All these file formats are quite different from each other and yet they contain partially common information. The structure files supplied by GROGRA containing information concerning morphological and topological structure and architecture of the plant are very accurate and clear, and thus easily transferable. Environmental information, on the other hand, is difficult 
to represent, as it is often encoded by functions and so-called sensitive growth rules, i.e. different programming paradigms are mixed (KURTH, 2002). However, through constant development GROGRA already provides a number of conversion interfaces to different software systems representing diverse models.

\begin{tabular}{|c|c|c|c|c|}
\hline & $\begin{array}{c}\text { GROGRA } \\
\text { (KURTH, 1994) }\end{array}$ & $\begin{array}{c}\text { AMAP } \\
\text { (DE REFFYE, } \\
\text { ET AL. 1995) }\end{array}$ & $\begin{array}{c}\text { HYDRA } \\
\text { (FRÜH, 1995) }\end{array}$ & $\begin{array}{c}\text { MIR/MuSc } \\
\text { (DAUZAT ET AL., } \\
1999 \text { ) }\end{array}$ \\
\hline $\begin{array}{l}\text { Structure data } \\
\text { (advanced level) }\end{array}$ & $\begin{array}{l}* .1 \text { sy } \\
* \text { *ssy } \\
*\end{array}$ & *.mtg & & \\
\hline $\begin{array}{l}\text { Structure data } \\
\text { (basic level) }\end{array}$ & $\begin{array}{l}* . d t d \\
* . d t g\end{array}$ & $\begin{array}{l}* \text { *lig } \\
\text { *.arc } \\
\text { *.brc } \\
\text { *.sce }\end{array}$ & $\begin{array}{l}* . m a p \\
* . p b g \\
* . s b g\end{array}$ & $\begin{array}{l}\text { *.top } \\
\text { *.scn } \\
\text { *.scu }\end{array}$ \\
\hline $\begin{array}{l}\text { Supplementary } \\
\text { Data }\end{array}$ & & $\begin{array}{l}* . \text { inf } \\
\text { *.dta }\end{array}$ & *.eva & $\begin{array}{l}* \text { *.met } \\
\text { *.lum }\end{array}$ \\
\hline
\end{tabular}

Table 3-2: Plant models to be interfaced with each other, together with their respective data file formats. The basic structure files describe the main part of the models. Advanced structure files, on the other hand, are expansions to the original formats based on higher mathematical concepts (formal grammars, graph theory). Supplementary data describe the general environment and framework of the simulated scenarios.

Although the interconnection of plant models is the principal objective of NEXUS, the program is not able to create or read L-systems using the formats $l s y$ and ssy of GROGRA. The development of such systems is not object of this thesis and thus these formats and the formalisms of L-systems cannot be included here. However, for the coding of plant structures the dtd format introduced in section 3.1.3 is essential.

AMAP, on the other side, provides a wide amount of information, both about the plant and its environment. This information is distributed over different files: geometrical information files, topological information files, parameter files, visualisation files. Due to the commercial use and distribution of the AMAP software, there is also a large complex of coded files, centred around the so called "linetree files", which are principally not public and thus difficult to use or access with other models. The programs AIR and MIR/MuSc are based on these and derived file formats. The files *.lig, *.arc respective *.brc describe the architecture of the plant and are only available as binary files and thus cannot be edited or modified by users. The files *.dta describe the characteristics of the graphical symbols used to represent the plant and can be edited and partially altered. The files *.inf contain information about the symbols for internal use. Editing and altering these files is not recommended, because they are attuned 
to the other non-editable files and changes can cause error while using the software. Through the co-operation between CIRAD and the University of Göttingen, the workgroup of W. Kurth had limited access to the internal formats and procedures used in AMAP. However, due to the security standards of the software to grant commercial efficiency the procedures and formats used are not up-to-date. At the moment, the interconnection is only possible with an older version of the AMAP system (version 2.2).

The development at the CIRAD of a new format based on multiscale tree graphs (MTG) leads to a reorganisation of the information management. The MTG format is used especially with AML, the AMAP Modelling Language (Godin AND CARAGLIO, 1998; GodIN ET AL., 1999a; GODIN ET AL., 1999b; GODIN, 2000) and is still in development, but is becoming more and more significant for the software development at CIRAD. The most important characteristic of this format is that it can contain in one file different description levels and scales for plant structures required for different special models. Hence this format seems optimal for data transfer. As mentioned above, an interface between AMAP and LIGNUM (PERTTUNEN ET AL., 1996) using the MTG format has been developed (DZIERZON AND KURTH, 2002), and GROGRA already possesses an unidirectional interface and is able to produce simple MTG structures, however it cannot read or process them. Through using the MTG format, NEXUS is supposed to overcome the version problem described above.

The file format sce is used to describe a scene with Landmaker software. A scene file is generated automatically after constructing the scene with Landmaker. The file is saved in ASCII format; however it is not recommendable to change it manually. The software group MIR/MuSc works on the basis of scene files. However, these programs don't need all the information found in sce-files and thus work with a simplified format called scn. Only structural data is retained, while visualisation data (camera position, light sources, etc) are discarded. This is made automatically. The working scene taken from the general scene described in the $s c n$-file is delimited concretely in the $s c u$-files, which also contain the geographical co-ordinates of the scene (latitude and longitude) needed to calculate the position of the sun.

The file format is: 


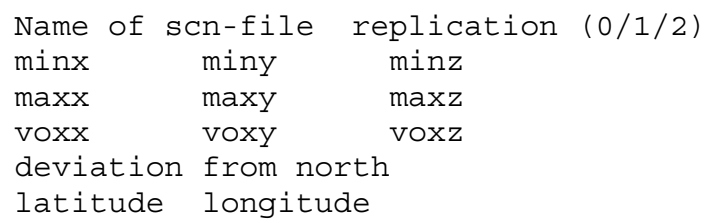

For example ejk.scu:

$\begin{array}{lll}e j k & 0 & \\ -15 & -30 & -15 \\ 30 & 15 & 30 \\ 2 & 2 & 2 \\ 0.0 & & \\ 52.75 & 10.5 & \end{array}$

The name of the scn-file is normally used to generate other files while running MIR/MuSc. The option "replication" indicates whether the scene is duplicated to simulate the surrounding environment or not. 0 means no duplication, 1 means that each plant of the scene is duplicated to create the environment. 2 means that all plants outside of the scene are ignored. Only plants that cross the borders are kept.

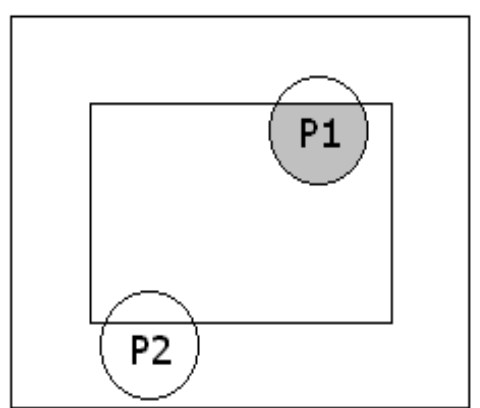

Option 1

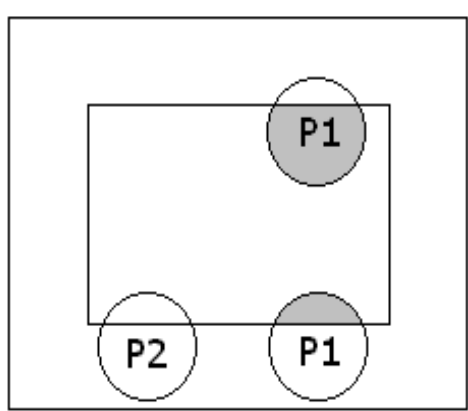

Option 2

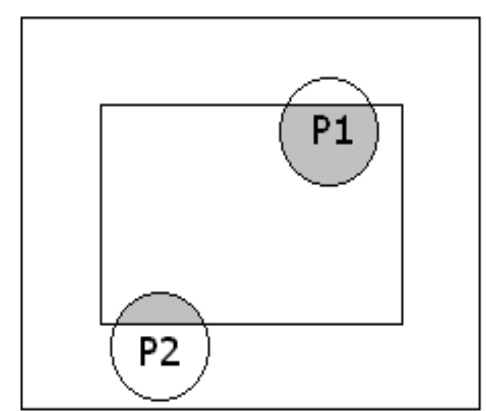

Option 3

Figure 3-12: Replication modes used in scu-files. In option $0, \mathrm{P} 2$ is ignored because it is outside the used scene. In option 2, P2 is still ignored, but P1 is duplicated to create the surrounding environment. In option 3, P2 is used as part of the surrounding environment.

The parameters $\min x, \min y, \min z, \max x, \max y$ and $\max z$ are the boundary co-ordinates of the scene, voxx, voxy and voxz indicate the number of divisions in each direction used to create voxels. The longitude value can be set to 0 because the actual versions of the programs don't use it actually. The option is left open for future possibilities.

The parameters included in the $s c n$-file correspond to the parameters from the original scefiles from AMAP. With output detail 1, the plant is considered one single organ. With output detail 2, the calculations are made for each organ. 
The format is:

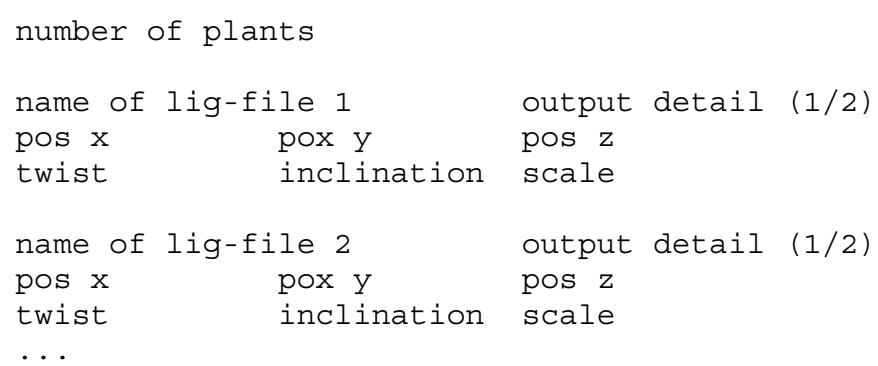

For example ejk.scn with only one plant:

1

$\begin{array}{lll}\text { ejk.lig } & 2 & \\ 44.3781 & 0.0 & 0.7\end{array}$

The met-files contain the meteorological data used to calculate the radiation balance and the sap flow. The file contains: date (Julian), time, air temperature $\left[{ }^{\circ} \mathrm{C}\right]$, PAR $\left[\mathrm{W} / \mathrm{m}^{2}\right]$, global radiation $\left[\mathrm{W} / \mathrm{m}^{2}\right]$, wind velocity $[\mathrm{m} / \mathrm{s}]$ and relative air humidity $[\%]$. The files .lum and .top are generated automatically and are for internal use only. The programs from the ARCHIMED shell (see 2.1.4.1) are being continuously actualised and developed, so that minor changes in the formats could occur.

The files .pbg, .sbg, .eva and .map of HYDRA are described in FRÜH, 1995. NEXUS generates automatically pbg-files as part of the interface with HYDRA.

Finally, the AMAP configuration file .cfg was expanded to create NEXUS configuration files, also .cfg, and species files .spc, which control some options for MIR, MuSc, HYDRO, HYDRA, GROGRA and NEXUS. The format of these files is:

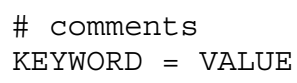

The new or expanded keywords of the cfg file are:

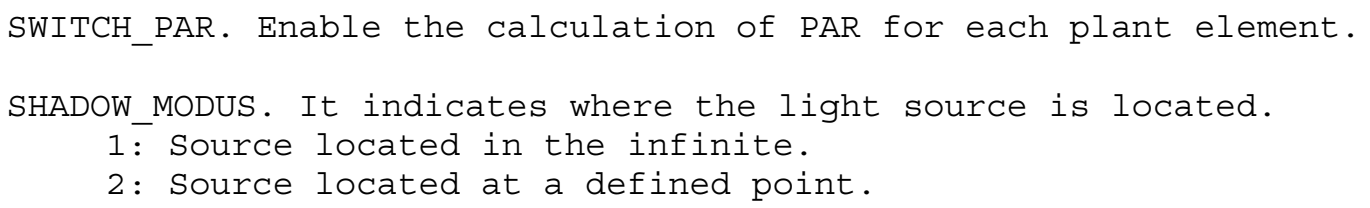


IMAGES. Directory for the resulting image files (.rgbn and.sgi for AMAP and .xpm for NEXUS). Default is the root directory.

METEO. Directory for meteorological data files (.met). Default is the root directory.

PROGRAMME. Directory containing the growth engines. Here it can also contain the path leading to the GROGRA directory. Default is the root directory.

SCENE. Directory for scene files (.sce for AMAP, .scu and.scn for NEXUS and ARCHIMED). Default is the root directory.

LAPS. Duration of the first time step in seconds. It must be greater than zero. The duration of the other time steps depends of the data found in the meteorological files.

NR PIXELS. Standard number of pixels in one scene. Recommended are $50 \overline{0} .000$ till 1.000 .000 pixels for better resolution.

TIME_CHANGE - Time zone used to calculate the position of the sun.

For the species files the parameters are:

AMAX. Maximal $\mathrm{CO}_{2}$ assimilation rate in $\left[\mu \mathrm{mol} \mathrm{CO}_{2} / \mathrm{m}^{2} \mathrm{~s}\right]$

AX_ALPHA and AX_BETA. Parameters for Conductance/Diameter Relation for branches: $\mathrm{KM}=\alpha \star \mathrm{D}^{\beta}$

AX_CAV_ALPHA and AX_WP. Parameters for cavitation function in the axes: $\mathrm{CON}=\overline{1} /\left(1+e^{(\alpha * \bar{T}-\mathrm{wp}))}\right)$

CAPA_LEAF. Water capacity of leaves in [kg / $\left.\mathrm{Mpa}^{*} \mathrm{~m}^{2}\right]$

CAPA_WOOD. Water capacity of wood tissue in [ $\left.\mathrm{kg} / \mathrm{Mpa}^{*} \mathrm{dm}^{3}\right]$

CO2_ATM. $\mathrm{CO}_{2}$ concentration in the atmosphere in $\left[\mu \mathrm{mol} \mathrm{CO}_{2} / \mathrm{mol}\right.$ air]

DECIDUOUs. Type of plant: 1 Deciduous / 0 Coniferous

EMAX_KG. Maximal transpiration rate in $\left[\mathrm{kg} \mathrm{H}_{2} \mathrm{O} / \mathrm{m}^{2}{ }^{*} \mathrm{~s}\right]$

EMAX_MOL. Maximal transpiration rate in [mmol $\mathrm{H}_{2} \mathrm{O} / \mathrm{m}^{2}{ }^{*} \mathrm{~s}$ ]

GH_ALPHA, GH_BETA, GH_XO and GH_YO. Parameters in the function for humidity dependence of stomatal conductance: $\mathrm{f}(\mathrm{dH})=\mathrm{y} 0+\alpha /\left(1+\mathrm{e}^{\{-(\mathrm{dH}-}\right.$ $\mathrm{x} 0\} / \beta)$ )

GMAX. Maximal stomatal conductance in $\left[\mathrm{mmol} / \mathrm{m}^{2} * \mathrm{~s}\right]$

GT_ALPHA, GT_BETA, GT_TMIN and GT_TMAX. Parameters in the function for the dependence of stomatal conductance upon leaf temperature $t$ : $f(t)=$ $\alpha *(t-t \min ) *(t \max -t)^{\beta}$

PET_ALPHA and PET_BETA. Parameters for Conductance/Leaf Area Relation for petioles: $\mathrm{KM}=\alpha \star \mathrm{LA}^{\beta}$ 
PET_CAV_ALPHA and PET_WP. Parameters for cavitation function in the petioles: $\operatorname{CON}=1 /\left(\overline{1}+\mathrm{e}^{(\alpha *(\Psi-\mathrm{wp}))}\right)$

SPECIES. Name of the species

\subsection{Programming techniques}

The modular character of the software requires the use of modern programming methods, especially the techniques of object oriented programming (OOP). With these techniques it is easier to implement a system of interchangeable modules, each representing a single special process to be modelled. SAARENMAA (1992) proposes a general object-oriented approach for tree modelling. Saarenmaa asserts that using an object-oriented approach is valuable for connecting function and structure. The structure is given by the internal division of elements into objects, which at the same time are connected to functions through tasks. Some processes can also be defined as objects on their own. Object oriented approaches allow a major degree of abstraction. The resulting classes of objects are often more intuitive and related to realworld concepts than computer concepts. There are two phases in the development of objectoriented systems (SALMINEN ET AL., 1994):

- Analysis (OOA). Description of information with real-world objects.

- Design (OOD). Expansion of the basic objects created with OOA by adding procedural and technical objects.

OOP is based on the concept of classes, objects, instances and inheritance. An object is a basic entity containing attributes and eventually methods describing its general behaviour. Methods are functions for the manipulation of the data contained in an object, and for the interaction with other objects. Ideally, the manipulation of the attributes of an object should be only possible by its methods. The description of such structures containing both data and methods is called class. Both, attributes and methods of a class are called member elements (member variables and member functions). After describing a class, an object can be created as an instance of this class. For example:

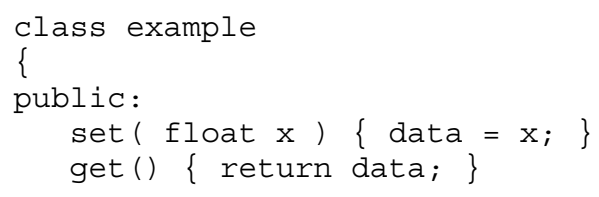




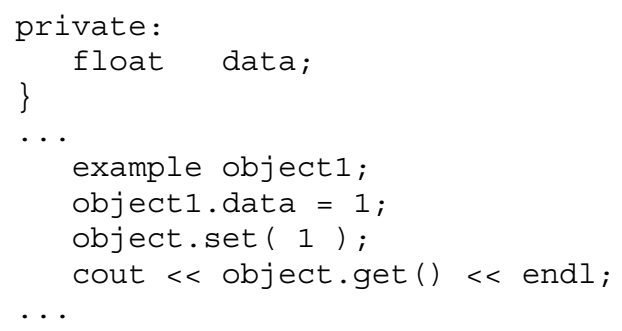

This very simple class example (written in $\mathrm{C}++$ ) defines an object containing an attribute data, and two methods, set for input and get for output. With "example object1;" an instance of the class example is created. The command "object1. data $=1$ " produces an error because the member variable data can only be handled through the member methods set and get described in the class definition. The user needs only to know which methods are accessible and how they are used. The implementation within the class remains "invisible" so that internal changes in a class can be made without the necessity that the user changes his code. The user handles only the objects themselves and creates his own instances. Some classes cannot have any instances. These classes are called abstract. They serve to define basic sets of attributes and methods which often need to be specified to single tasks.

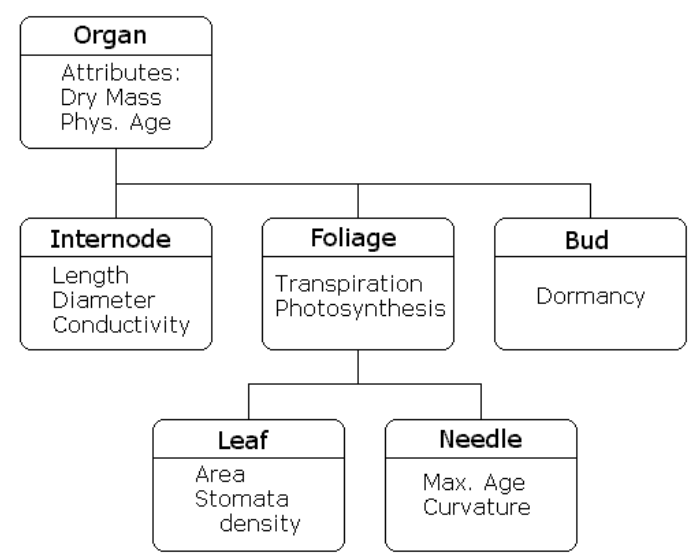

Figure 3-13: Example for class hierarchy and inheritance. Organ is the base class for Internode, Foliage and Bud. Leaf and Needle are derived from Foliage. A Needle object would have a curvature value, a maximal age (by itself), transpiration and photosynthesis rates (from Foliage), and dry mass and physiological age (from Organ).

A class can be generated by deriving it from existing classes. The original class is called base class or super class, the new class subclass or derived class. Abstract classes can only be used as base classes. A derived class gains the attributes and methods of it base class. This is called inheritance. Derived classes expand the gained set of member elements to fit new needs, and can on their own be used as base classes. Through the inheritance over many levels of classes a hierarchy is created. Classes at the top of a hierarchy contain elements that are common to 
all their subclasses. Classes at the bottom of the hierarchy contain very specific member elements and are thus more specialised. In some cases a class can be derived from more than one base class. This is called multiple inheritance. Finally, a special form of functions and classes called templates can be defined. A function-template is a function whose arguments have not a specific type but a type parameter. The needed type is instanced during the call of the function according to the type passed by the argument. This allows to define a function, which could work with different types, only once. For example a function $\min \left(\begin{array}{l}T \\ a\end{array}, T\right.$ b) with the type parameter $\mathrm{T}$ would work with integer, float or double values. A class template is a class with type parameters that serve as type for members of that class. Class templates are often used to define container classes like lists and vectors.

The implementation of general classes for the different universal compartments of the plant and its environment is the first step. These form the set of structural classes that can be expanded according to the requirements of future models. The representation of these classes can be reached easily using the already mentioned MTG format. Thus the representation and comparison of a simply-structured model with the compartments "plant", "soil" and "atmosphere" with a more complicated and highly structured model, such as the one provided by GROGRA with compartments for each growth unit, becomes possible. SAARENMAA ET AL. (1994) and SALMINEN ET AL. (1994) present the framework of an object-oriented tree model to describe forest health. BRECKLING (1994) describes the application of object-oriented models in plant ecology with the background of modelling single plants (individual based modelling).

On the other hand, a set of process oriented classes will also be developed. Most of the currently existing models for whole plants or stands function by using a set of smaller, often fixed, models for single and specific processes, together forming a sort of model complex. The integration of the basic models in a set of process oriented classes makes them easier to exchange and to compare. This step of model integration is only possible with a good cooperation between the workgroups, as it will often mean a reorganisation of the original model implementations. At this level, NEXUS acts as a comparison tool for process model specifications. The comparison of models with different temporal scales remains a main but not yet realised objective. The most important and central objective of NEXUS is the development of the model feedback with the growth engines. 
The decision to use the OOP techniques led to the use of the programming language $\mathrm{C}++$. $\mathrm{C}++$ is widely available and provides by means of the Standard Template Libraries (STL) an extensive set of programming tools that facilitate the co-operation between the different programmers. Most of the already existing models described here have been implemented using $\mathrm{C} / \mathrm{C}++$. Besides the STL provided by Silicon Graphics, the cross-platform $\mathrm{C}++\mathrm{GUI}$ application framework Qt from Trolltech was used. Qt is supported on Windows, Unix/X11 and Macintosh platforms. This is important, because a further aspect of the software development is its platform independence, as the final software should be available for both UNIX and WINDOWS systems. Programming was made on three platforms: on an SGI IRIX terminal, on Windows 95 using Borland $\mathrm{C}++5$ and MS Visual $\mathrm{C}++$, and on Linux SUSE 6.2 using KDevelop.
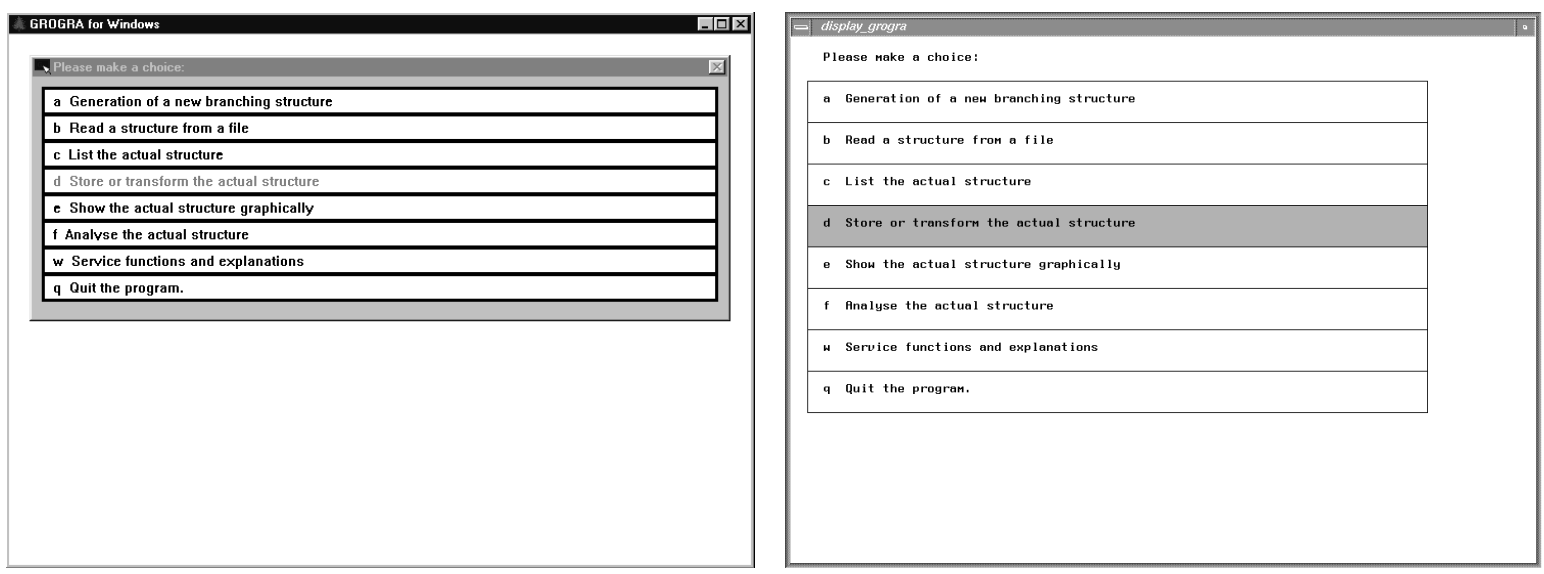

Figure 3-14: Screenshots from WGROGRA (left) and XGROGRA for SGI IRIX systems (right). The pictures show the same menu in both versions with modified gray tones.

In the first stage of the project, a Windows-compatible version of GROGRA, called WGROGRA, was developed and tested in order to warrant that this growth engine works on the most common platforms, as there were already executable versions for DOS, IRIX and XWINDOWS (UNIX systems). Figure 3-14 shows a screenshot of WGROGRA and its counterpart for UNIX. Due to backward compatibility and maintainability needs, WGROGRA could not be written using all the possibilities from Windows programming, especially file and window management. WGROGRA was written in Borland $\mathrm{C}++$ but without using OOP, because most of the files and libraries provided by Kurth, the author of the original version of GROGRA, are written procedurally in C. However, the availability of WGROGRA was important for the development of the project and for the collaboration with other workgroups. 
Next, the incorporation of the AIR software into NEXUS was to be realised. This software provides a first interconnection between AMAP and HYDRA and can be run individually or from an already running program. This option was designed for starting AIR from AMAP. AIR reads the structure files from AMAP and generates an sbg-file (Secondary Base Grid), which is used in HYDRA to calculate the water flow. The growth feedback with AMAP was not realised at that time. As the HYDRA software originally works only with some species of conifers, the AIR software also supported only the same parameters. With the new data from oak, both software packages HYDRA and NEXUS had to be adapted to work with deciduous plants, too. The adaptation of HYDRA has been done by M. Schulte from the University of Göttingen.

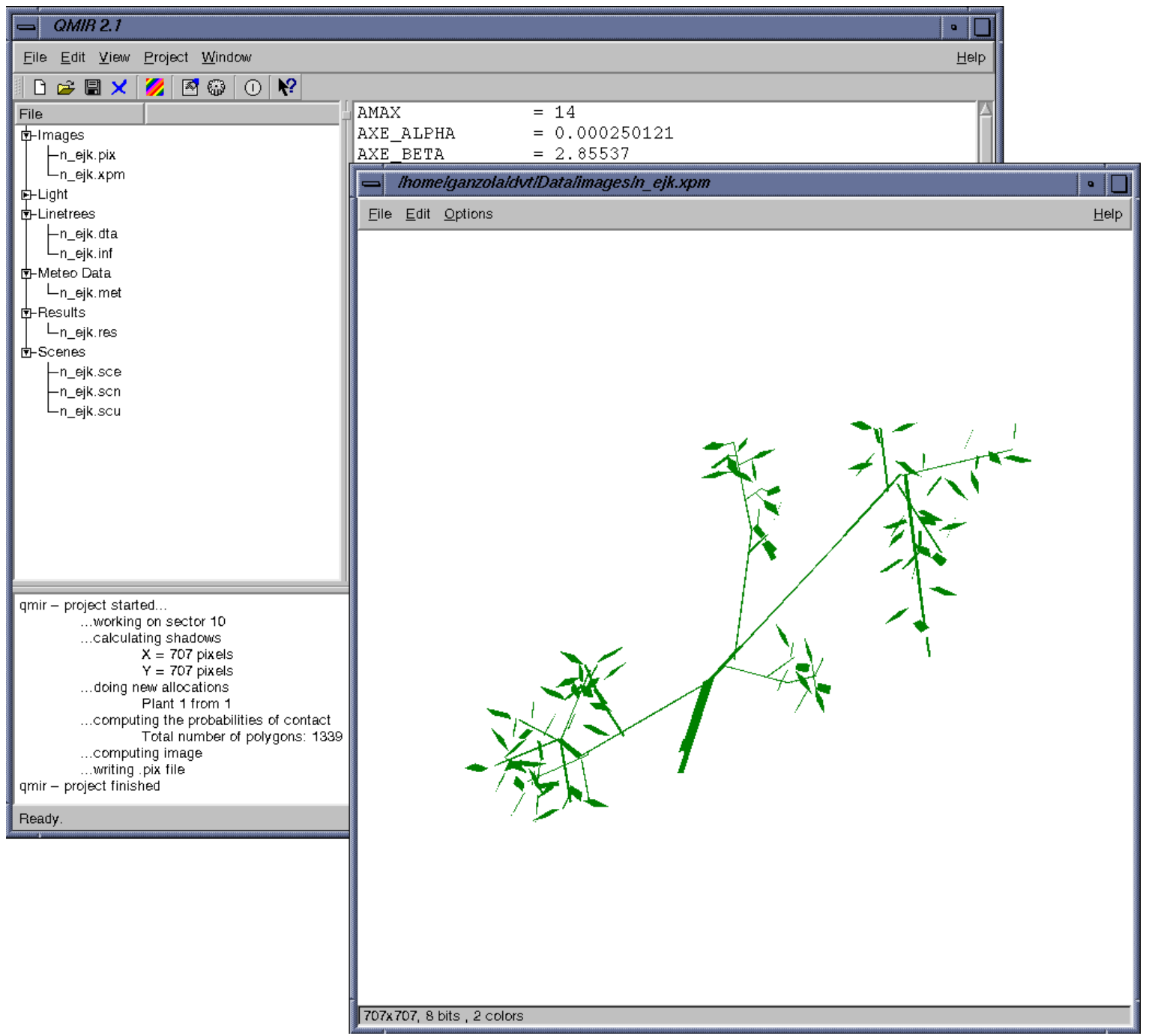

Figure 3-15: Screeshot of QMIR with its visualisation tool. The picture represents tree ejk viewed from sector 10 of the "turtle". The resolution of the picture depends od the number of pixels selected in the configuration file. Original colors have been changed for better reproduction. 
In co-operation with J. Dauzat and Ch. Nouguier from CIRAD, France, the MIR/MuSc software has been adapted for NEXUS, writing new $\mathrm{C}++$ versions according to the principles of OOP. Using the Qt libraries, the programs QMIR and QMuSc have been developed. Currently, a new version of MIR is being created at the CIRAD, in which the number of sectors is not fixed to 46 but variable, so that it can be adapted to different situations (see section 2.1.4.1). A free standing plant with few leaves (and thus with low self shadowing) needs fewer sectors than a highly structured plant in an environment with frequent irradiation changes. Besides these two radiation simulation programs, the water flow program HYDRO was tested as alternative to HYDRA. Again, an object-oriented version called QHYDRO has been developed. HYDRO and HYDRA form the first two modules for water flow to be included in NEXUS. The idea is to combine the numeric stability of HYDRA with the more exact calculation of leaf transpiration of HYDRO. Due to some differences in the boundary conditions, the setting of time intervals and the spatial discretisation of both programs, some difficulties arose in the combination of both programs.

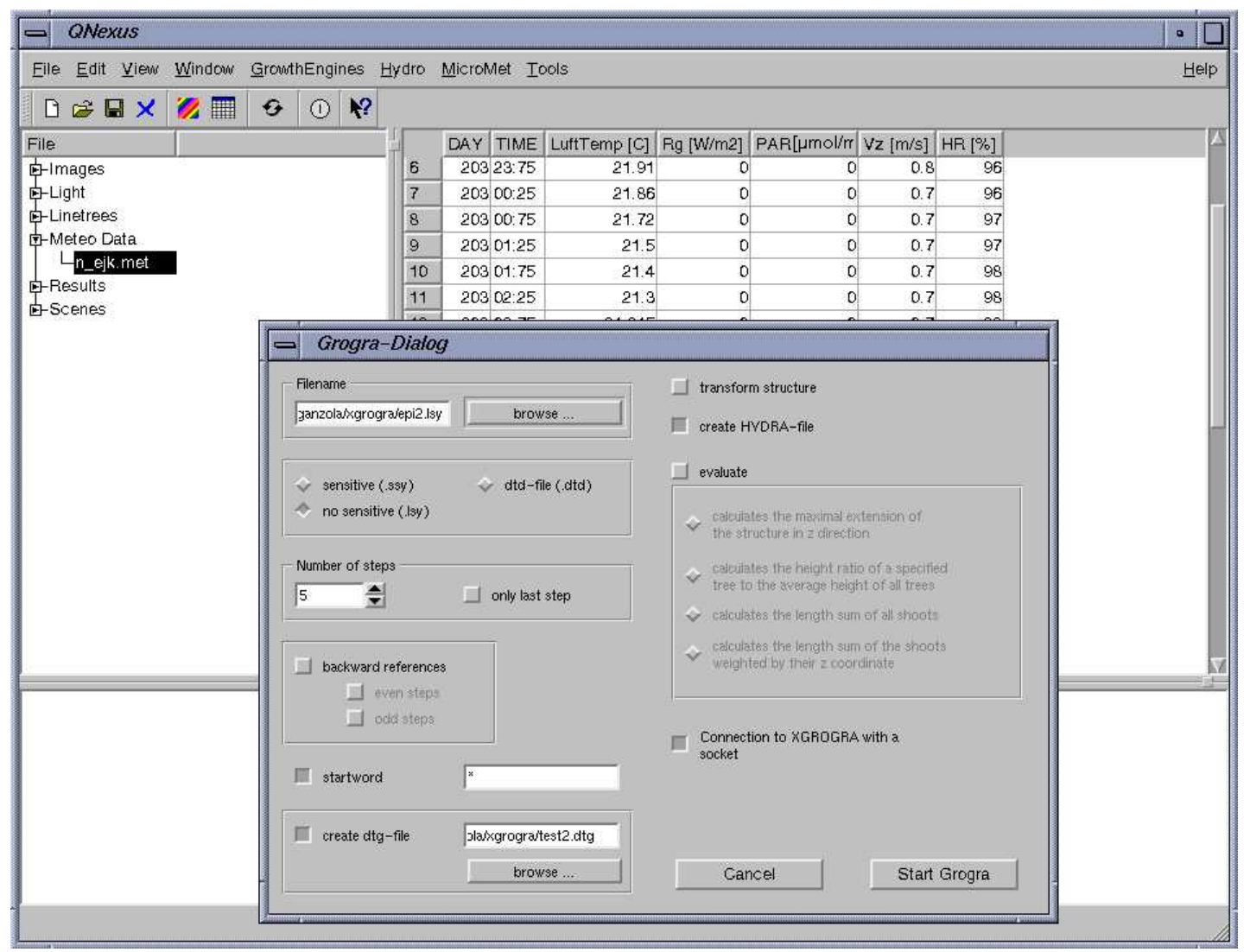

Figure 3-16: Screenshot of QNEXUS displaying the dialog for starting GROGRA. Every command line parameter can be selected in this dialog. With a special option, GROGRA runs interconnected with NEXUS via socket. 
The shell NEXUS itself was also programmed in C++ using both STL (LIPPMAN AND LAJOIE, 1998) and Qt (LEHNER, 2001). During the design phase, the standard templates and classes from RogueWave were proposed for the project. The classes from RogueWave are however only commercially available and thus more difficult to obtain and make open source programming difficult. For this reason one objective set while writing the software was to create a set of portable tools and standard classes to be used by several co-operation partners. These tools and classes include mathematical and geometrical functions, file management (independent from Qt, STL and RogueWave) and standard templates. These classes are to be shared with the workgroup AMAP at the CIRAD.

The interconnection with the growth engines and with the other models has been realised partially through a set of socket based interfaces. The programs GROGRA, MIR, MuSc and HYDRO can be called automatically according to project data, or NEXUS can used as a launch platform where the user directly selects the options for running the respective programs. Also a series of editing tools for the different (editable) files has been created.

The software has been documented using the program "Doxygen" to generate the API documentation, including class hierarchy, inheritance and member description in form of html files, which will be posted online after the software if finished. "Doxygen" is a documentation system based on LATEX, which can extract information from the code structure to generate on-line documentation (html) and off-line reference manuals in LATEX, RTF, PDF, PS or UNIX man pages format (VAN HEESCH, 2001).

To make it easier for other programmers to read and understand the code, there are a few guidelines to follow when writing $\mathrm{C}++$ code.

\section{Identifier names:}

- The name of an identifier should not be an abbreviation, unless it is the normally used name, e.g. PAR (Photosynthetically Active Radiation) or NIR (Near Infra-Red). Identifiers like nvin (non valid identifier name) should be avoided.

- Global variables begin with "the” (for example, theApplication). 
- Constants begin with lowercase c (for example, cMaxwindow).

- Global constants are written all in uppercase (for example, SCREENLINES). Globally defined variables are used instead of macros for allowing a better type control.

- Class data members begin normally with _ (for example,_symbol).

- Function parameters end with _ (for example, filename_). Ideally, a passed object name_ initialises a member object_name. This allows a better control of passed values.

- Static data members begin and end with _ (for example, _total_).

- Non-member functions begin with an uppercase letter (for example, Textstyle() ).

- Member functions begin with a lowercase letter (for example, getName () ).

- Names of classes begin with project identifier (for example, nxClass). Typical identifiers are Q for Qt classes, RW for Rogue Wave classes and K for KDE classes. KDE is another GUI based class library based on Qt. The identifier nx was selected for NEXUS.

\section{CLASS DECLARATIONS}

Classes are declared in the following order:

public member functions

protected member functions

private member functions

protected data members

private data members.

No data members should be declared public. For example:

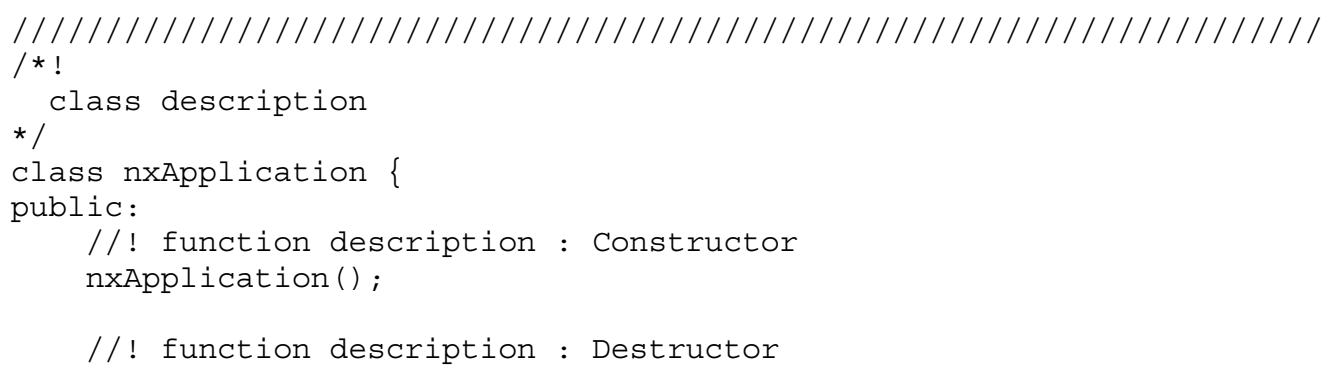




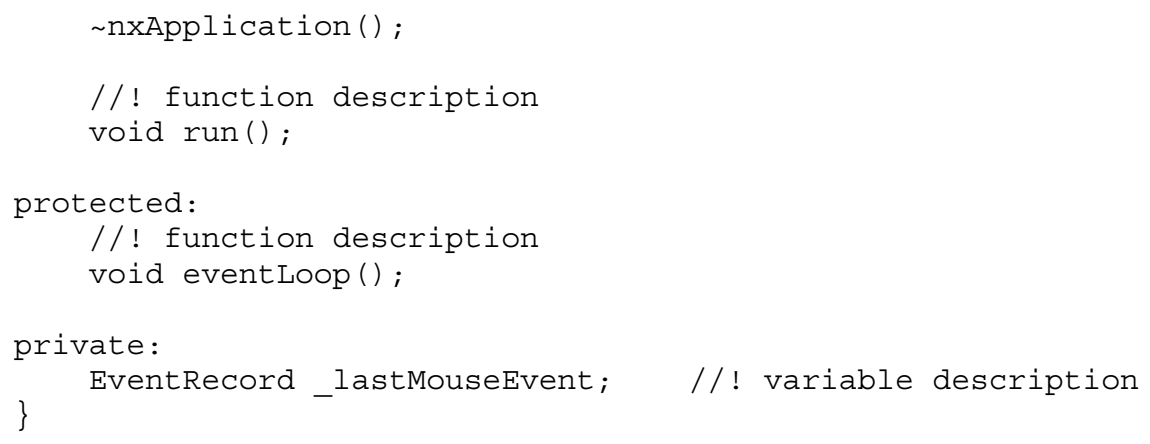

The comments used in form $/ * ! \ldots * /$ and $/ /$ ! are used to create data that can be read by Doxygen to create the documentation (VAN HEESCH, 2001).

Control structures: The control structures are written as follows:

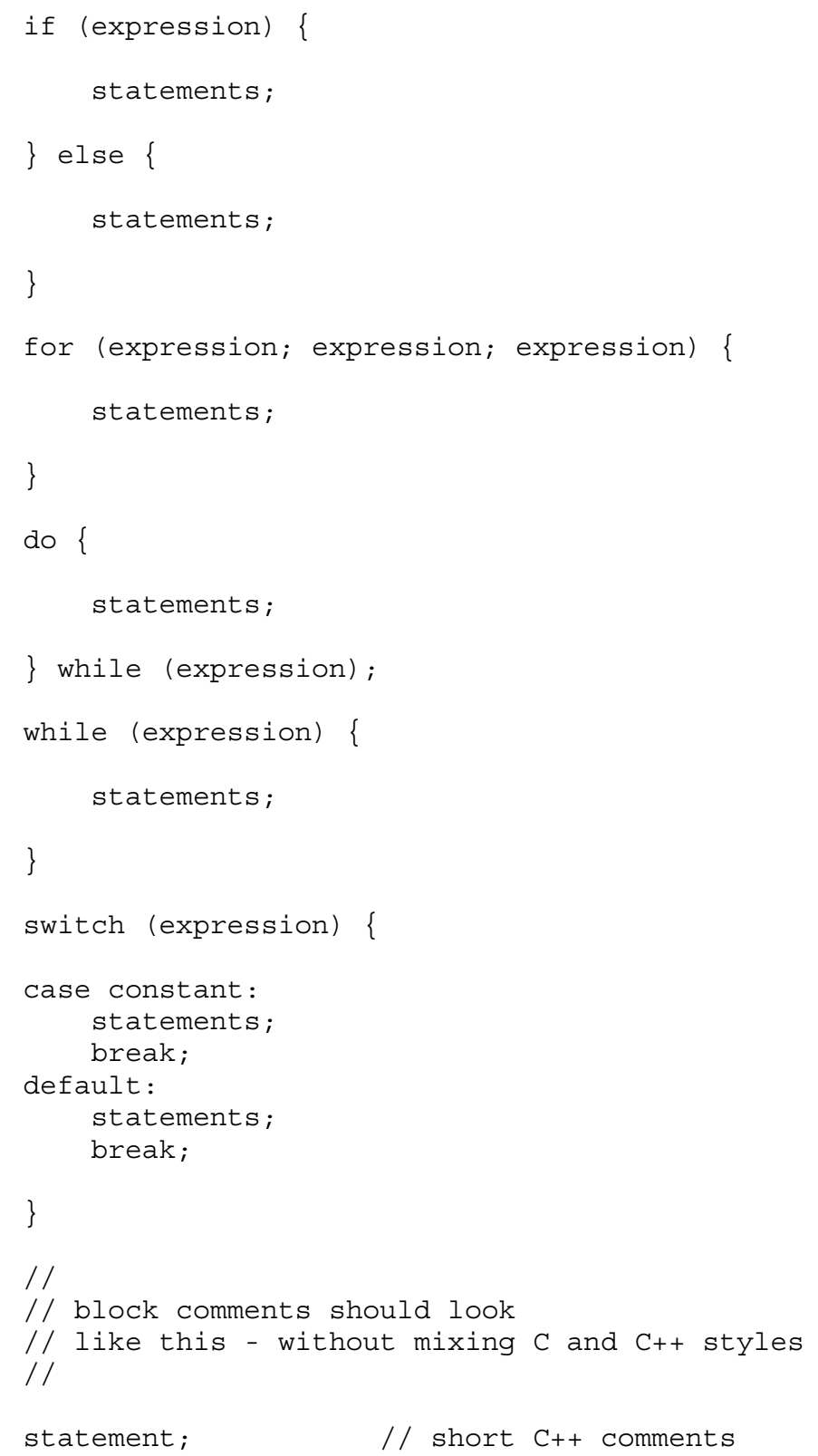




\section{THE SHELL PROGRAM NEXUS}

NEXUS was conceived as a platform independent shell for plant model interconnection. The idea is to provide a tool that handles different models as interchangeable modules. In general, any process and architecture model that is specified generically can be used as a module. Functional-structural models that handle only specific processes can also be integrated as modules. For other more complex models, NEXUS should act as an interface tool for accessing the provided models.

The major difficulty in achieving these goals is the problem of accessing background data and "insider" information to the models. Process models as presented in section 2.1 are often well defined, so that they may be included easily. In this case, the major difficulty rests in the parameterisation.

Structure models, on the other side, are often very rigid and inflexible. For that reason a generic structure model was chosen. Similar to some of the functional-structural models presented in section 2.3 and to the MTG format (GODIN ET AL., 1999a, 1999b) the structures in NEXUS are represented by nodes and internodes as basic unit. This was necessary to handle the different spatial scales found in different models. The structure information used in NEXUS is not used for external structures. NEXUS reads the structure data generated with other programs and creates new files for other models. The present version can read structure data generated with AMAP or GROGRA. As output, NEXUS generates $p b g$-files needed by HYDRA. The model can then read extended $p b g$-files generated with HYDRA to calculate new values in the next time step. The internal data structure contains the information for linking the data given in different formats. Figure 4-1 shows the differences between the representation of the structures with GROGRA, AMAP and for the input of HYDRA. 

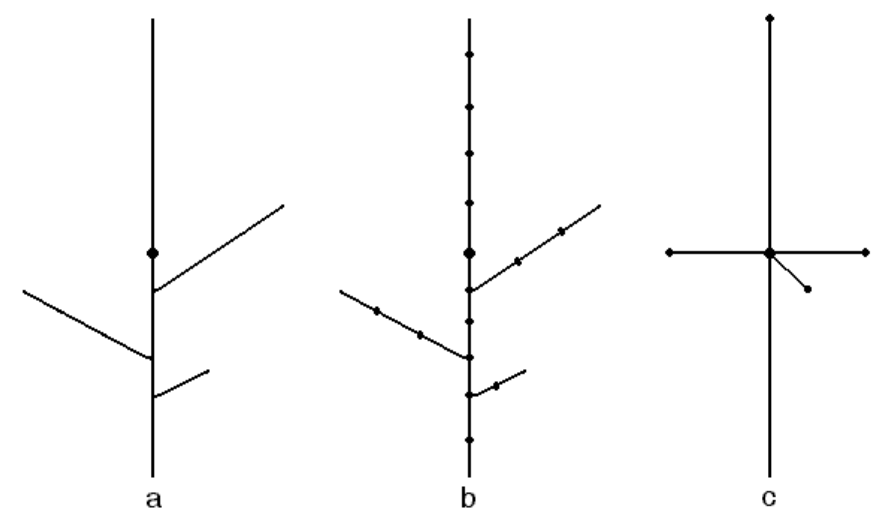

Figure 4-1: Representation of the tree structure in different formats. a) The standard model in GROGRA represents the structure at GU level. b) In AMAP, internodes and nodes are included in the structure files. c) In the pbg file from HYDRA, the structure is changed to meet the requirements for the numerical model. NEXUS keeps track of the relation between structures.

\subsection{The Graphical User Interface (GUI)}

As part of the initial project, a set of four programs has been written: NEXUS, HYDRO, MIR and MuSc. The last three programs were developed originally by Jean Dauzat at the CIRAD, France. However, it was necessary to make some changes to the existing programs to grant a better compatibility with other programs. The parameterisation of these programs was made for Coffea arabica (RAPIDEL, 1995) and was included within the code, so that every new parameterisation leads to a great amount of rewriting and fitting the programs anew. Furthermore, the programs were written procedurally. For reasons mentioned above, object oriented versions of the programs were necessary. There are two sets of programs. The first set contains text versions of the programs with very simple interfaces. These programs are provided for machines without x-server support and will not be maintained in the future.

The second set was programmed using the graphic support of Qt for XWindows applications. All four programs were written following the same scheme, which provides a similar graphical interface as the one used by KDevelop. Each program can be started individually and each supports a set of command line parameters for facilitating its use. And all programs can be started using the interface included in NEXUS. Figure 4-2 shows the general graphical user interface (GUI). The GUI contains standard menu and tool bars for file management, data editing and for specific characteristics of each application. Each program contains a project window, which allows a better control of information flow. In this project window the 
used files are shown following the classification similar to that of AMAP: image files, radiation files, linetree files containing the architecture and topological information of the trees (lig, arc and dta files), meteorological data, scene files (sce, scn and scu files) and result tables. The format of these files has been explained in section 3.2. On the right of the project window is an editor window. By double clicking on an item in the project window, the respective editor (text, table or dialog) is opened in this window. The only exception is the graphical view tool which works as an own application. Some items in the project cannot be edited, because it is not reasonable to do so, e.g. linetree files. The last window situated at the bottom of the application is an output window. Any temporal results, runtime messages and error warnings are written into this window. This window is not editable.

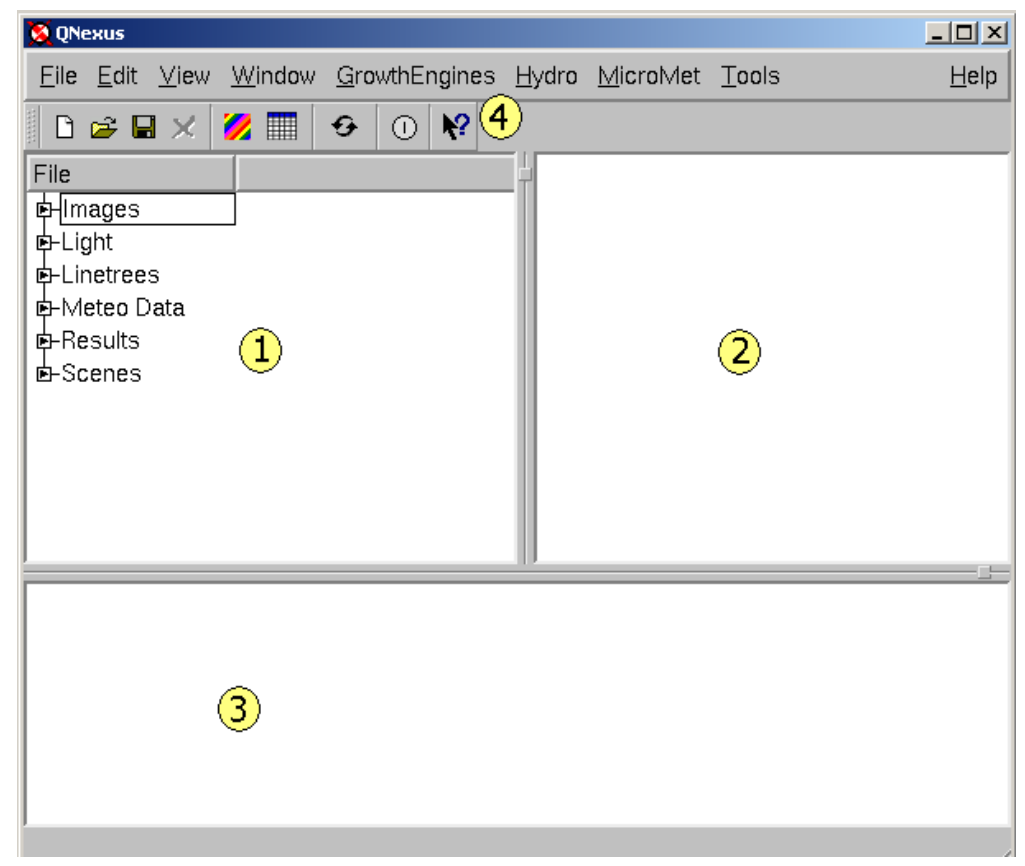

Figure 4-2: Generic graphical user interface (GUI) in the NEXUS project. 1) Project window. 2) Editor window. 3) Output window. 4) Menu and tool bars.

The most important menu items in NEXUS are:

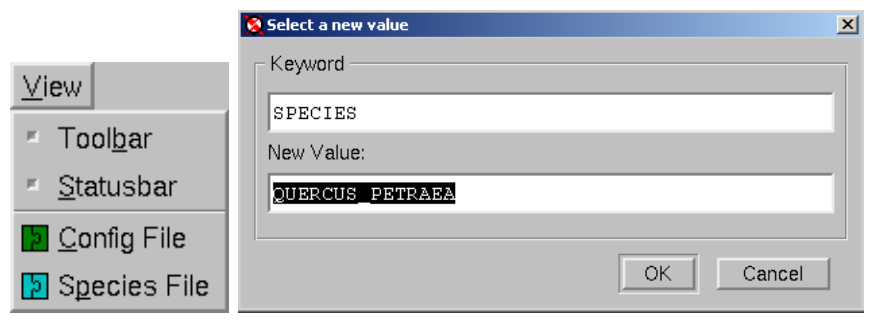

The view menu. This menu provides some options for the optical appearance of the GUI and gives access to the configuration and species files. The content of the configuration and species files appears as a list in the editor window. By double clicking an 
selecting an item an input dialog appears, where the user can directly select the command line parameters.

The command line form for MIR is qmir $[-\mathrm{h}] \quad[-\mathrm{s}<$ name $] \quad[-\mathrm{p}<$ name $] \quad[-\mathrm{t}<0-46>] \quad[-1$ <int $>] \quad[-0<0-4>]$. With:

$$
\begin{aligned}
& -\mathrm{h}, \quad-\text { help } \\
& -\mathrm{s}, \quad--\mathrm{scu}<\text { name }> \\
& -\mathrm{p}, \quad--\mathrm{XPM}<\text { name }> \\
& -t, \quad-\text { turtle }<0-46> \\
& -1, \quad-- \text { limits } \\
& -o, \quad-- \text { output }<0-4>
\end{aligned}
$$

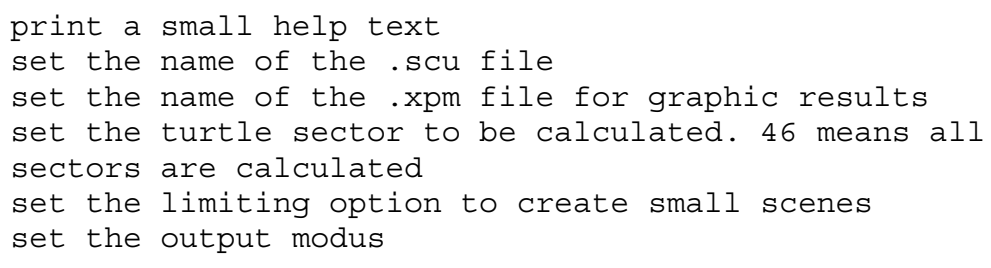

These parameters can be also selected after starting MIR.

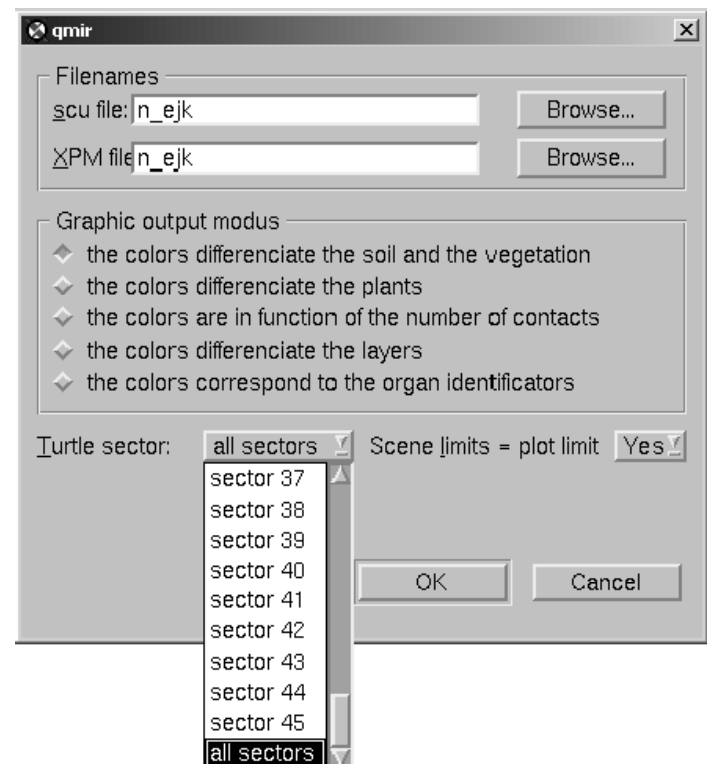

Figure 4-3: Parameter dialog of QMIR. The parameters can be selected directly in the command line. This dialog is also used in NEXUS to create the command line of MIR.

The command line form for MuSc is qmusc $[-\mathrm{p}<$ name> $] \quad[-1<$ int $>] \quad[-\mathrm{s}<$ int $>]$. With:

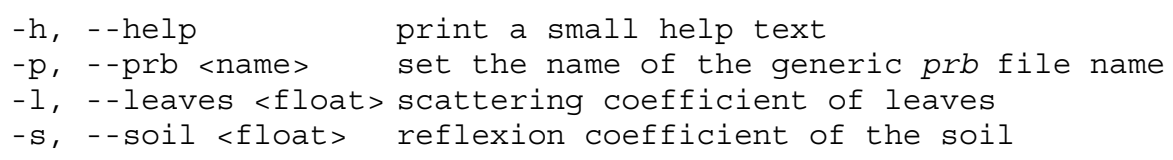

\begin{tabular}{l} 
Iools \\
\hline ViewPix \\
Iableview \\
SceneConvert
\end{tabular}

The tool menu. This menu contains different items for each application. The general items are a graphical view application, a table editor, and a text editor. With the graphical view application the user can load the output graphics, make minor modifications like changing colours, setting 
a monochromatic view, changing size etc., save and convert the graphics to other formats. Formats available are: bpm, jpeg, pbm, pgm, png, ppm, xbm and xpm. The standard format is $x p m$. This application is based on a standard graphics tool provided by Qt using OpenGL, from DEC, IBM, Intel, Microsoft and SGI (BARTH ET AL., 1996). The scene converter is a small application for converting sce files into scn files and vice versa.

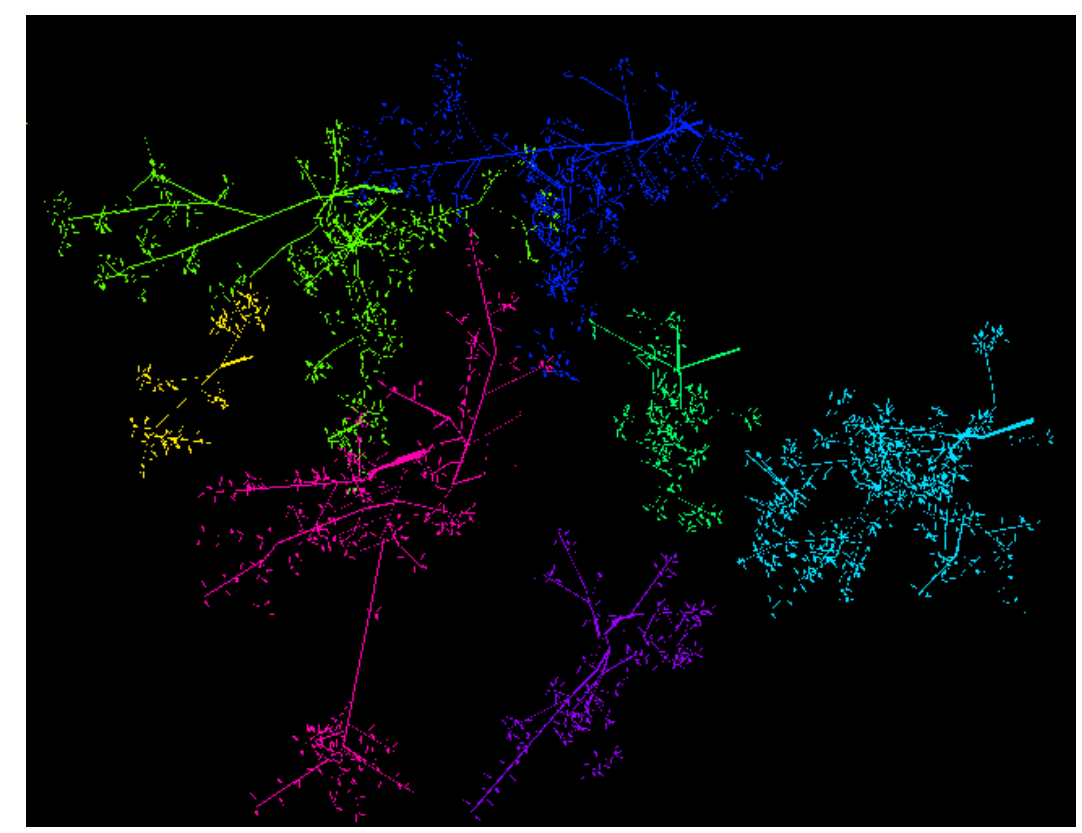

Figure 4-4: Picture generated by QMIR and viewed with the tool ViewPix. QMIR calculates the pixels that are hit by a light ray coming from an specific direction (ray tracing method). The output can follow different options. Here each plant has a different colour.

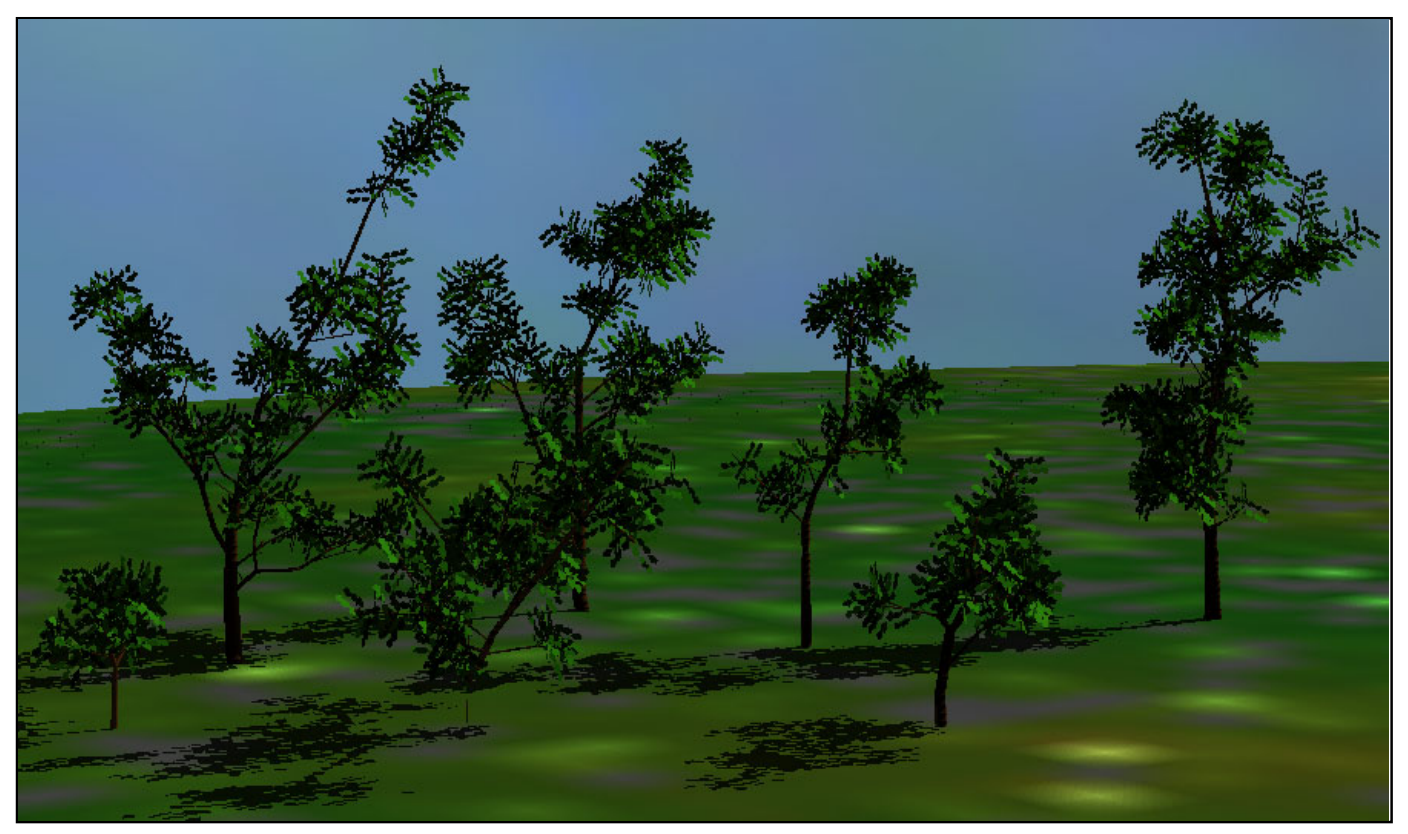

Figure 4-5: AMAP scene used to generate the picture in Figure 4-4. 


\subsection{Classes}

In the framework of object oriented programming, some generic classes were created. These classes are available as open source and will be used in future projects. Other classes are more specific and are not easy to transfer to other projects. These include the classes used for describing the architecture and the behaviour of the different organs.

Bifstream, derived from the STL class ifstream and bofstream derived from the STL class ofstream. These classes handle input and output of binary streams taking into account the endian code of the used machine. The endian code describes how the machine handles the high and low bytes for the binary representation of variables. The endian code must be known before instancing a stream object. These classes are provided for sharing files created in machines with different endian code.

nxFile is a more effective file handling class, as the user don't need to know about the endian code; it is determined automatically. This class is similar to the class ofile from Qt. In the Qt versions of the programs QFile was used instead of nxFile.

nxDate is a class for managing date in the Gregorian and the Julian calendars. The data input supports many formats and the output is also variable, e.g. dd/mm/yyyy or verbose version. A similar class, QDate is provided with Qt. This class has slightly different methods for handling the conversion Julian to Gregorian time and vice versa. In the Qt version QData has been used to maintain the same basis. Other classes are nxTime and nxDateTime for handling time in hours, minutes, seconds and milliseconds, and for day time respectively. Again, Qt provides the classes QTime and QDateTime, which were used in the Qt versions. The differences between the $\mathrm{nx}$ (NEXUS) versions and Q (Qt) versions are not really significant. The $\mathrm{nx}$ versions are used with the test versions of the software.

The class nxGeographics handles geographical co-ordinates and altitude. nxEphemeride combines nxGeographics with nxDateTime (or QdateTime) for calculating sun positions and times of sunrise and sunset. The class nxsun is related to nxEphemeride. 
The class nxpixel handles pixel co-ordinates and a series of attributes like colour. The nxImage class is a container of nxPixel. These classes are used to generate graphical results.

The class nxpoint handles a point with $\mathrm{x}, \mathrm{y}$ and $\mathrm{z}$ co-ordinates. The class nxVector is derived from nxPoint. nxPolygon is a list of nxPoint objects.

The class nxDocument handles files that are editable. The class $\mathrm{nxCfg}$ is derived from nxDocument and handles files with the format:

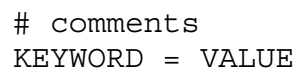

The classes nxConfig and nxSpecies are derived from nxCfg and handle the configuration and species files respectively.

The class nxImageviewer contains the graphical view application described above.

The class nxProject handles a generic project, and the class ePrjview handles the project window of the generic GUI. The class nxTable handles tabular data in the editor and the class nxTextEditor handles editable test data..

The class nxsceneConverter contains the scene converting tool described above.

The class nxScene handles a group of plants as generated with AMAP. A nxScene can also contain only one plant.

The class nxTurtle describes a turtle object as described by DEN DuLK (1989). The actual version is fixed to 46 sectors. A future version will handle a variable number of sectors.

The class nxRad handles the radiation balance of a scene. It contains the values for global, direct, diffuse and photosynthetically active radiation. The class nxTurtleRad is derived from nxRad and works with nxTurtle to calculate the radiation balance from each turtle sector. The class nxMusc handles the radiation from the multi-scattering sub-model. 
The class nxtine handles the information from the linetree files and reconstructs the tree architecture. It serves as a control class for the organ containers nxBranchList, nxSegmentList, nxuunctionList and nxLeafList.

The class nxplant handles a single plant with all its elements. The class nxorgan defines the basic characteristics of each organic element.

The classes nxLeaf, nxsegment and nxJoint are derived from nxorgan. The class nxBranch describes a growth unit. The class nxpipe describes a sequence of nxBranch objects.

The class nxerror is an error handling class. It delivers the error codes if an error can be identified.

Besides these classes, many mathematical classes and function templates were created that can substitute those provided by Qt or STL so that it is possible to create programs that are independent from them. These classes are mainly used in the text version of the programs mentioned.

The exact description of the classes and their members and methods will be available in form of on-line manuals. These will be posted initially at the server of the GWDG under http://www.gwdg.de/ ganzola/nexus.

Most classes have a number of undefined parameters in form of container classes of variable size. These are class templates (see 3.3) which are initialised with a base number of elements but that can be automatically expanded if more elements are used, or reduced if the number of elements decreases. With these "free" attributes, the classes can handle models with different parameters. The mean objective of the NEXUS project was to provide a possibility to interchange such sub-models. However, due to the problems with the parameterisation that represents an own problem, only the models provided by the original programs were tested. The idea is to create a collection of sub-models in form of modules that can be changed by simple selection in the program's menu. 
In relation to the models used originally the following changes were made: First, all parameters whose values were fixed within the code were substituted with variables. Constants were also defined separately in a special class called nxGlobal. All the constants are initialised with standard values, but it is possible to change their values for special cases. This can be achieved using parameter files like the species file described in section 3.2. Parameters that are normally variable are initialised with 0 and must be set by the user. Second, the function call for sub-models is kept general. The functions called provide the appropiate models according to the settings in the configuration files of the choices at run time.

NEXUS uses the event handling capacity of Qt to control the information flow between the programs. In this way, NEXUS can control the performance of HYDRA and GROGRA, which are programmed procedurally. GROGRA has already an interface for the socket connection, thus enabling a bi-directional information flow between, e.g., the radiation model (QMIR/QMuSc) and the growth engine (GROGRA). To use this, it is necessary to develop special L-systems that rely on this data flow. Although simple L-systems were tested, more complex models supporting radiation balance and water flow have not yet been realised.

The interconnection with the LIGNUM software (PERTTUNEN ET AL., 1998) and with AML (GoDIN, 2000) is being realised in a parallel project by Helge Dzierzon at the University of Göttingen. The interconnection with HYDRA depends on the parameterisation and adaptation of that model to Quercus spc. and has not yet been completely achieved till now. The adaptation of HYDRA is part of a parallel DFG-funded project carried out by Dr. Michael Schulte at the University of Göttingen. 


\section{Simulations}

In order to run the simulation with the programs MIR/MuSc and HYDRO, the collected architectural tree data were transcribed into the AMAP file format using GROGRA. The original $d t d$-files and their representation with GROGRA are documented in Appendix 1.

Once the tree structures were translated to AMAP, the single scenes were reconstructed from the measured data and graphical scenes were created using the tool Landmaker from AMAP 2.2 on an SGI IRIX workstation. The following figures show the created scenes.

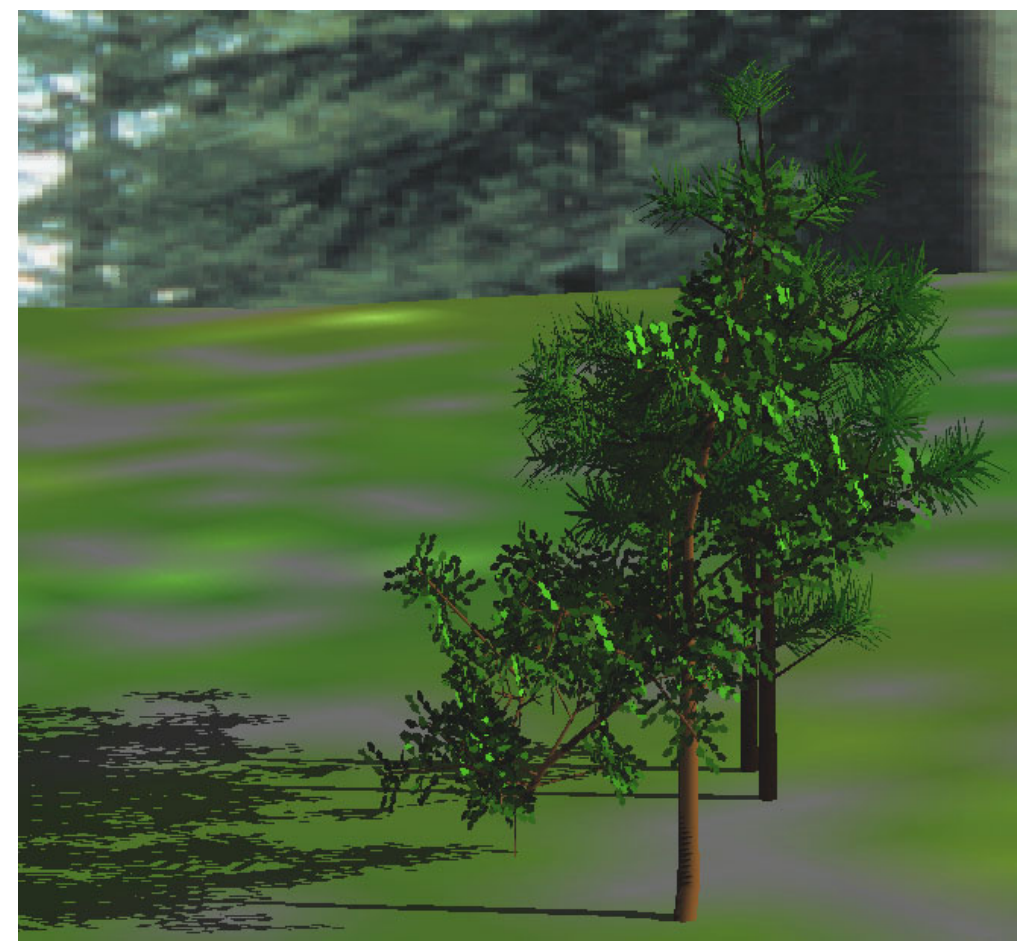

Figure 5-1: Scene for ejc. 


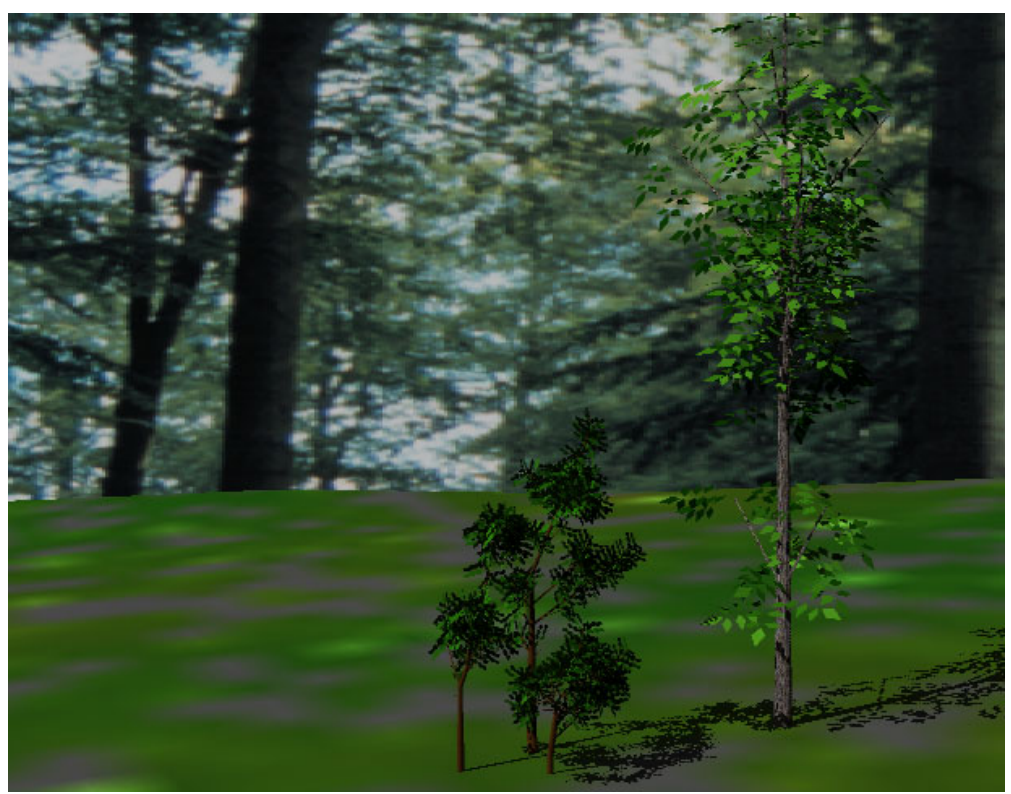

Figure 5-2: Scene for ejd.

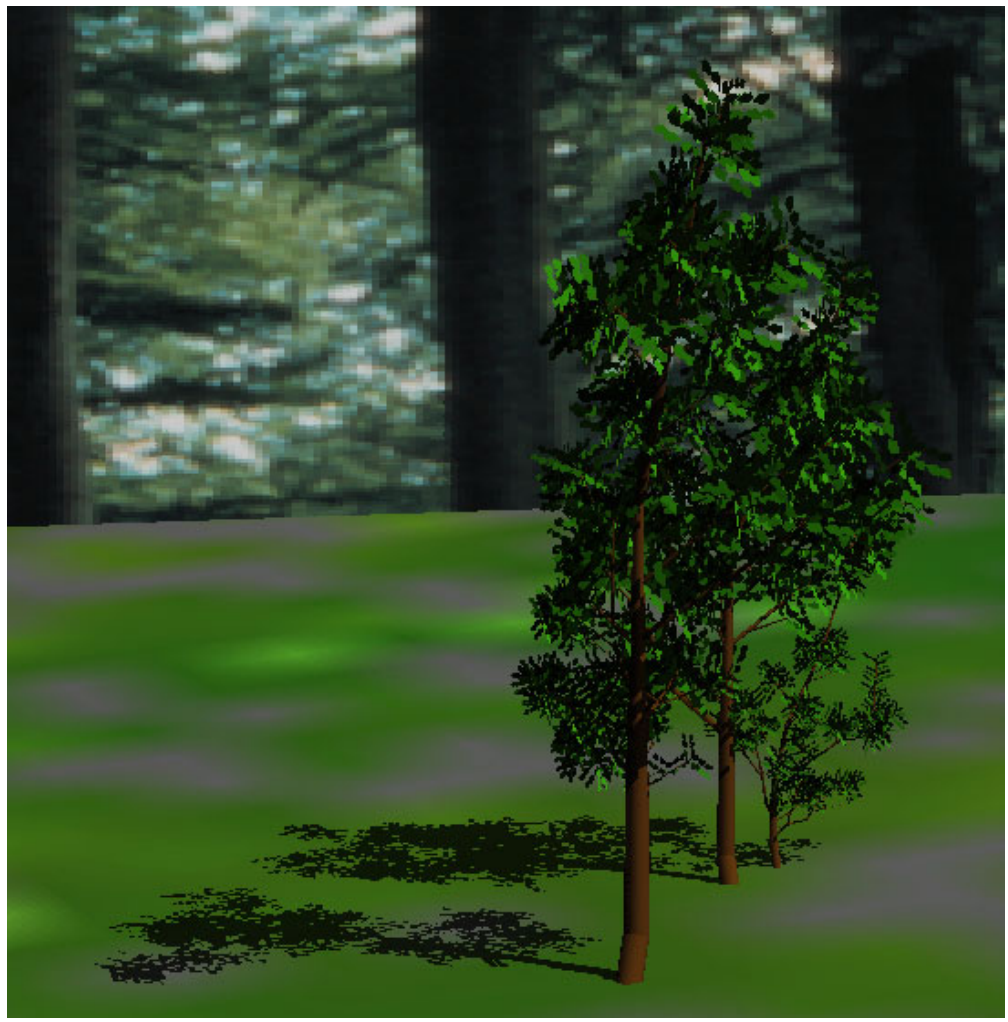

Figure 5-3: Scene for ejf. 


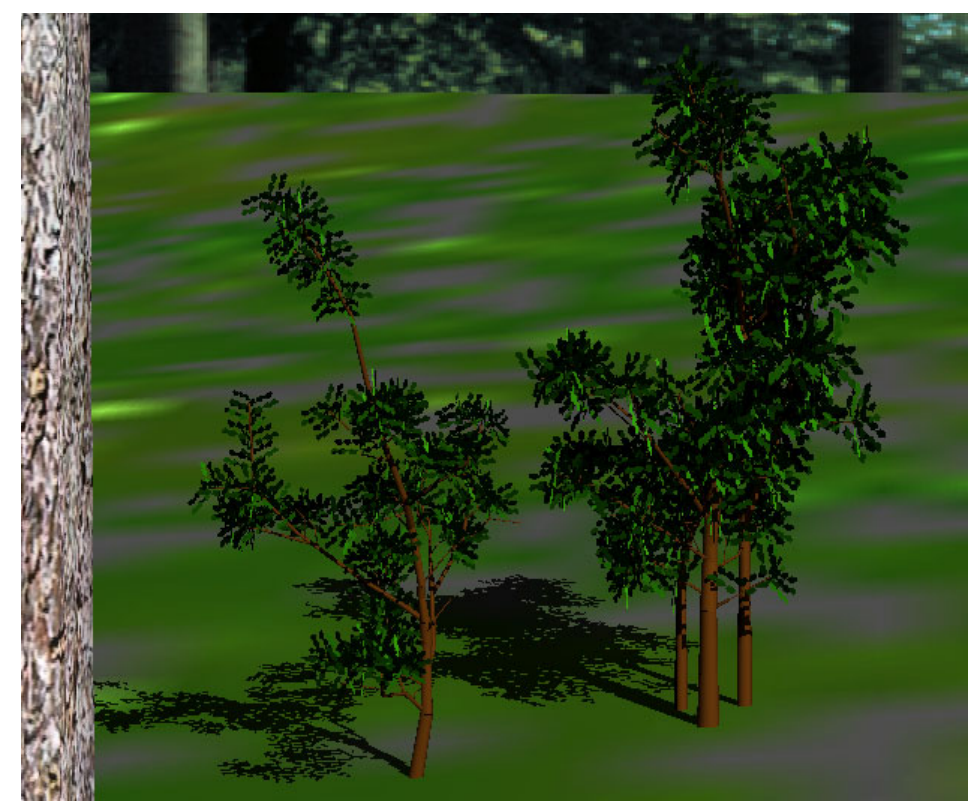

Figure 5-4: Scene for ejg.

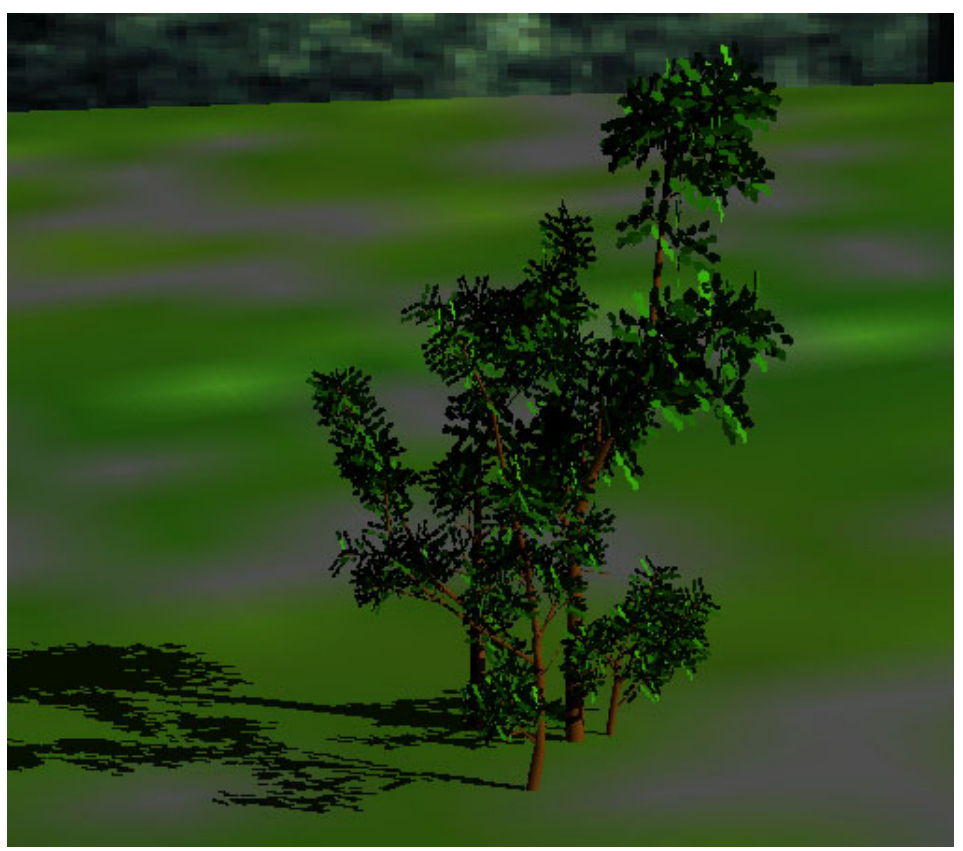

Figure 5-5: Scene for ejh. 


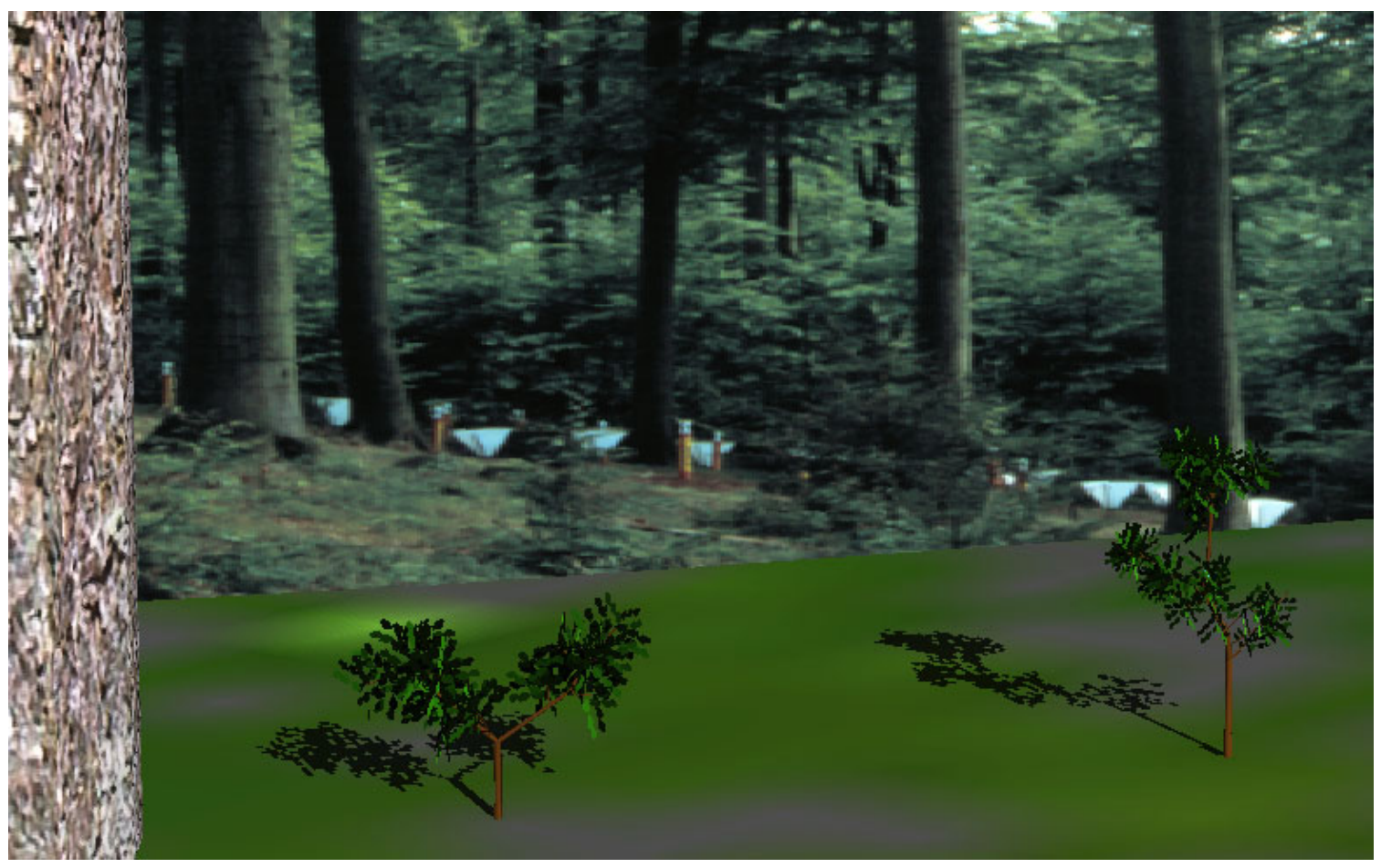

Figure 5-6: Scene for ejk.

Using the data provided by J. Dauzat, the initial validation of the new versions of MIR, MuSc and HYDRO was made. Due to some changes in the numerical handling of some values and functions the results obtained show a small variation in comparison with the original results. It also depends on the handling of the AMAP symbols. The original leaf symbols used by Dauzat and Rapidel were not available and different but similar symbols were used. Figure 5-7 shows the evolution of calculated transpiration values.

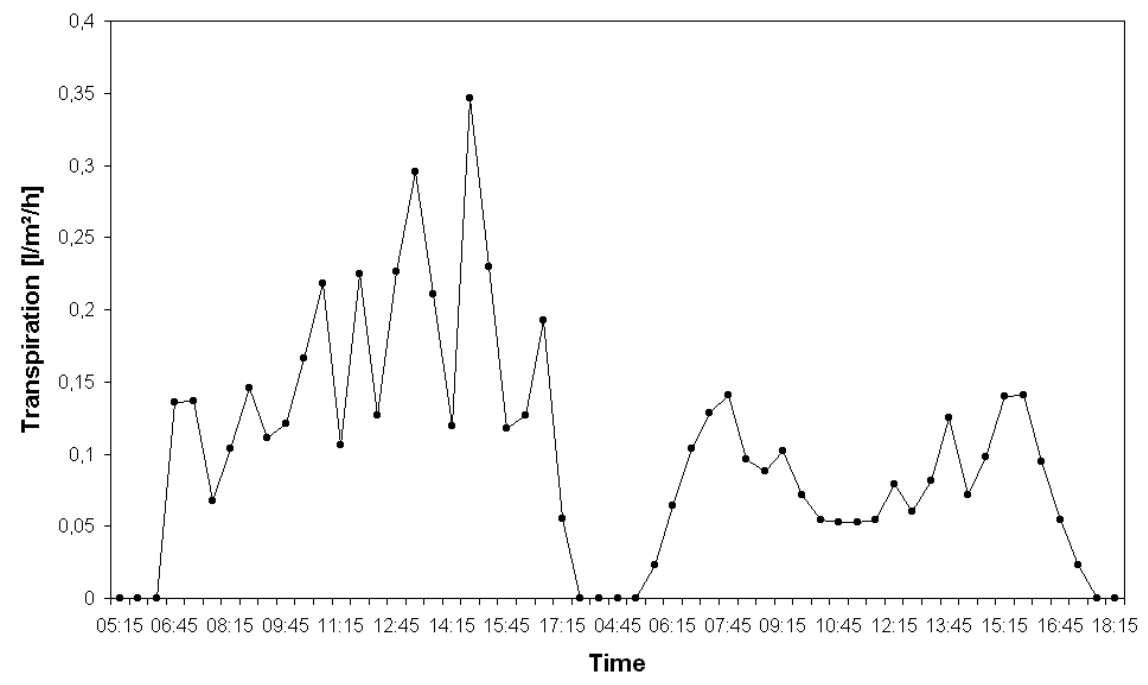

Figure 5-7: Evolution of calculated transpiration values of a coffee plant according to the meteorological data from Costa Rica provided by Dauzat (see 3.1.2). 
The original model still shows a minor bias (RAPIDEL, 1995) which not was corrected in this project. The model tends to underestimate leaf temperature at high PFD values. The temperatures were corrected by fitting the boundary layer resistance coefficients through a multiplication by 2 . The water flow is calculated correctly with some minor discrepancies. It is expected that the values calculated in combination with HYDRA will be more accurate. HYDRO tends to slightly underestimate the water potential gradients. HYDRA on the other side manages the hydraulic gradients more exactly due to its numerical stability but the absolute values of the hydraulic potentials are not yet optimally handled (SCHULTE, personal communication).

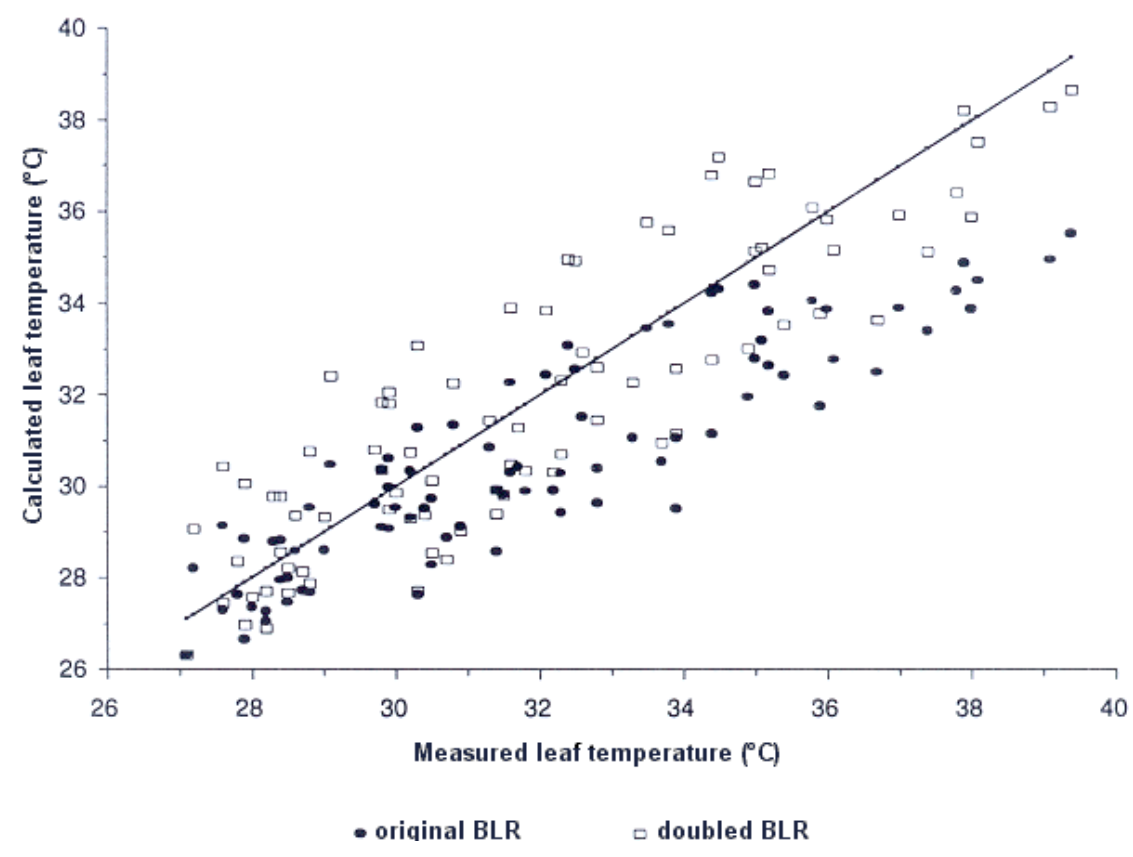

Figure 5-8: Comparison of measured and calculated leaf temperatures. The black symbols represent the original data, the white symbols represent the modified data after the multiplication of the boundary layer resistance (BLR) by 2 (After RAPIDEL, 1995).

As described above, the main problem resulting from the adaptation of the models to new plant species is the parameterisation of the used functions. The first simulations with the oak trees were made using the meteorological data from Costa Rica and the same parameters. Figure 5-8 shows the results of such a simulation made with the tree ejk. The parameters used in these simulations are: $\mathrm{g}_{\max }=450 \mathrm{mmol} \mathrm{m}^{-2} \mathrm{~s}^{-1} ; \mathrm{PFD}_{1 / 2}=100 \mu \mathrm{E} \mathrm{m}^{-2} \mathrm{~s}^{-1}$; el $=17$; e2 = 15; $\mathrm{T}_{\min }=15{ }^{\circ} \mathrm{C} ; \mathrm{T}_{\text {opt }}=24{ }^{\circ} \mathrm{C} ; \mathrm{T}_{\max }=41{ }^{\circ} \mathrm{C} ; \Psi=-1.3 \mathrm{MPa}$ and $\Psi_{\max }=-2.3 \mathrm{MPa}$. These parameters are used for the multiplicative stomatal sub-model used (JARVIS, 1976; compare also section 2.1.2). The obtained values for the oak were much lower than the values for the 
coffee plant, probably because of the lower number of assimilation organs. For other trees the values seemed to be too high (SCHULTE, personal communication). As the oak trees were run with the meteorological data from Germany, significant errors occurred. The most obvious error was caused by the temperature regression used by Dauzat and Rapidel. Because $54 \%$ of measured temperatures are $15^{\circ} \mathrm{C}$ or less, the values provided were negative (or 0 for $15^{\circ} \mathrm{C}$ ), and thus the whole equation for the stomatal conductance produced negative values. This was corrected by setting $\mathrm{T}_{\min }$ to $0{ }^{\circ} \mathrm{C}$, and leaving $\mathrm{T}_{\mathrm{opt}}$ and $\mathrm{T}_{\max }$ as given. These values were not measured or fitted, they were only set to avoid the negative values.

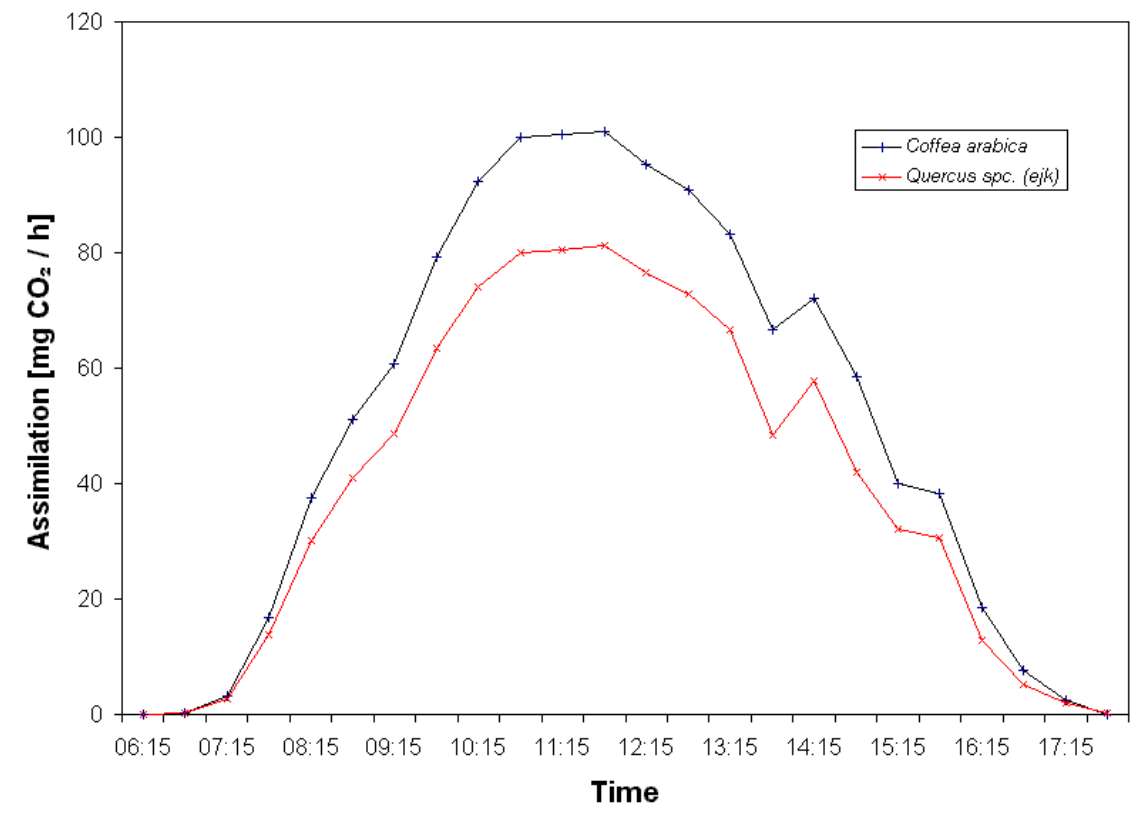

Figure 5-9: Results of the first simulation of the coffee plant and tree ejk using meteorological data from Costa Rica. The diagram shows the assimilation for April 27, 1994.

Most of the parameter fitting was made by Michael Schulte. The HYDRA software was parameterised for some tree species: Pinus sylvestris, Abies alba, Cedrus atlantica, Cedrus deodora, Thuja occidentalis, Pseudotsuga menziesii, Juglans regia, Coffea arabica, Fagus silvatica and Quercus rubra. Sufficient data for Quercus petraea and Quercus robur were not yet available. Furthermore, The modifications of HYDRA for broad-leaved trees are not yet finished. In coniferous trees, all the needles in a segment are handled as one unit. For leaves another solution was necessary. The parameters resulting from this first fitting are:

$$
\mathrm{g}_{\max }=420 \mathrm{mmol} \mathrm{m}^{-2} \mathrm{~s}^{-1}
$$




$$
\begin{aligned}
& \mathrm{PFD}_{1 / 2}=25.181 \mu \mathrm{E} \mathrm{m}^{-2} \mathrm{~s}^{-1} \\
& \mathrm{~T}_{\text {min }}=8.49{ }^{\circ} \mathrm{C} \\
& \mathrm{T}_{\mathrm{opt}}=24^{\circ} \mathrm{C} \\
& \mathrm{T}_{\max }=37^{\circ} \mathrm{C}
\end{aligned}
$$

A part of this fitting procedure includes determining values for petioles and leaves to improve the calculation of water flow in these organs.

Figure 5-10 shows the relation of sap flow to some meteorological data. The meteorological data from Unterlüß were then used to calculate new water flow and assimilation values. The wind velocity was not measured in Unterlüß. The values for wind velocity from Costa Rica were used to substitute the missing data from Unterlüß.
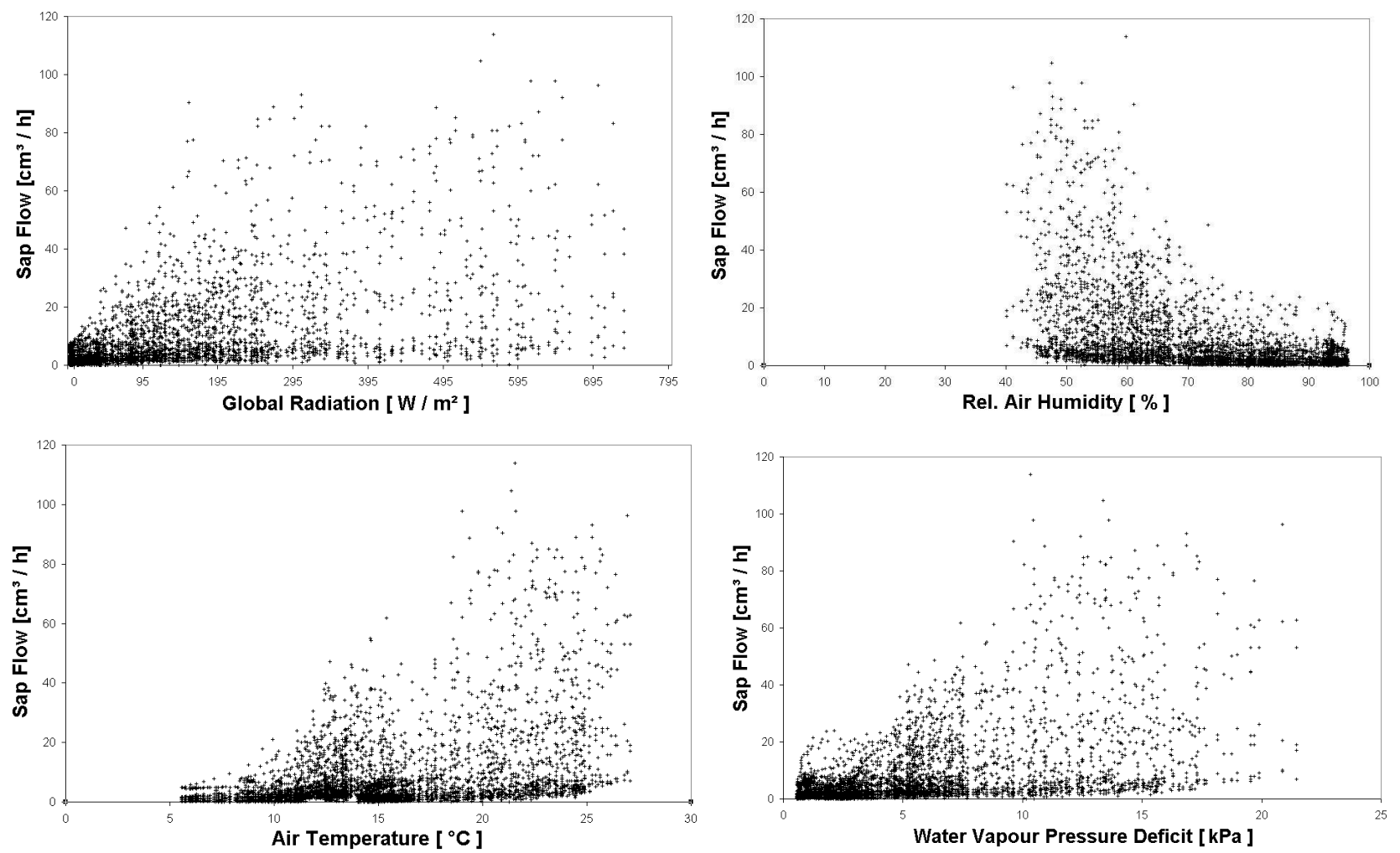

Figure 5-10: Sap flow of all sampling trees in relation to meteorological data: Global radiation, relative air humidity, air temperature and air vapour pressure deficit. The lower flow rates correspond to the smaller trees, ejk, ejk and ejh. The upper part of the scatter-plot correspond to the larger trees ejf and eji. The flow rate depends upon the respiration surface, and thus upon the number of leaves, and overall conductance. 
One problem with the wind velocity is that the sampling plot has a very complex structure. The wind model used in HYDRO leads to very different results depending of the "extra" trees included in a scene. In most of the scenes only small trees were considered to be relevant. The effect of wind on photosynthesis can vary. On the one side, high wind velocities induce changes of leaf surface temperature, on the other hand it reduces the thickness of the boundary layer. Which effect is predominant depends on the species. Due to the changes mentioned above to regulate leaf surface temperature, the real effect of wind cannot be exactly estimated.

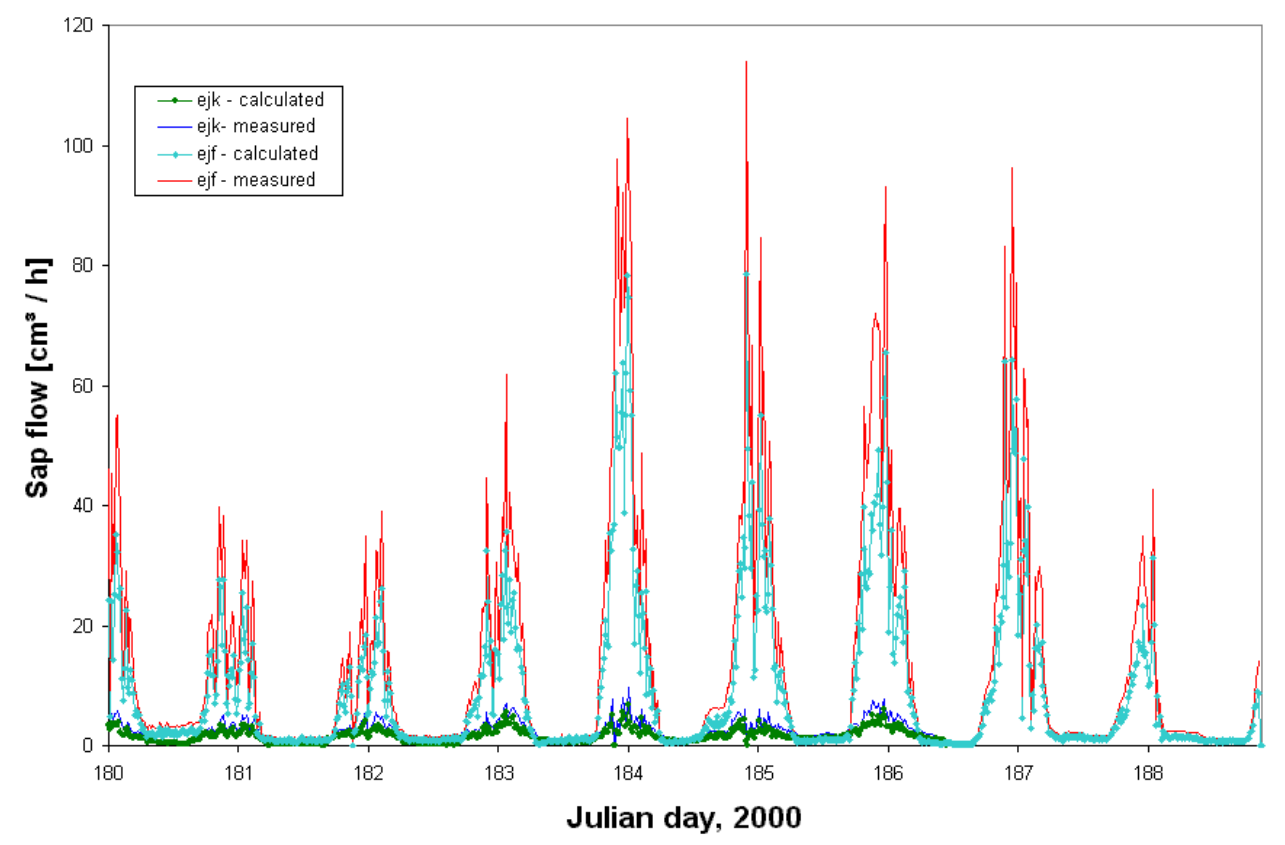

Figure 5-11: Measured and calculated sap flows for the trees ejk and ejf. Calculated values tend to underestimate the measured values by up to $20 \%$ divergence.

The water flow values calculated tend to underestimate the measured values. The discrepancies reach up to $20 \%$ below the real values. However, the general shape of the evolution curve is met well. Besides the difficulties of the parameterisation, another cause for the discrepancies can be the mentioned inhomogeneity of the sampling plot which is very difficult to reproduce accurately.

While the sap flow nearly matches the measured values, the assimilation values calculated seem to be too low, especially compared with the values obtained before. This can be caused by the lower radiation input in Unterlüß, but also on some bias in the parameterisation. 


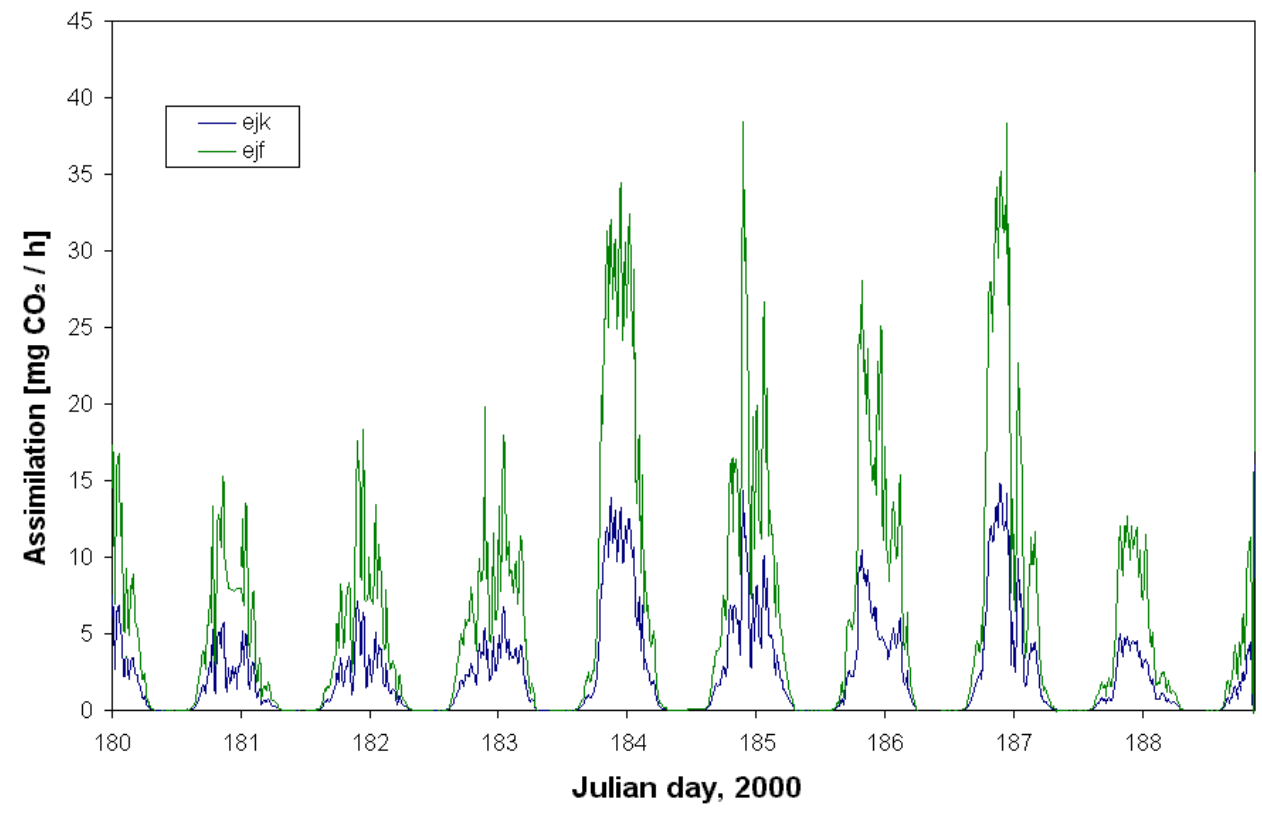

Figure 5-12: Assimilation of trees ejk and ejf.

Although exact values cannot be calculated at the current state of parameterisation, the qualitative comparison of the trees with each other can be made. More important is that the qualitative reaction of the trees to different growth environments can be shown. The assimilation values from solitary trees are higher than those of the same trees located amongst the rejuvenation.

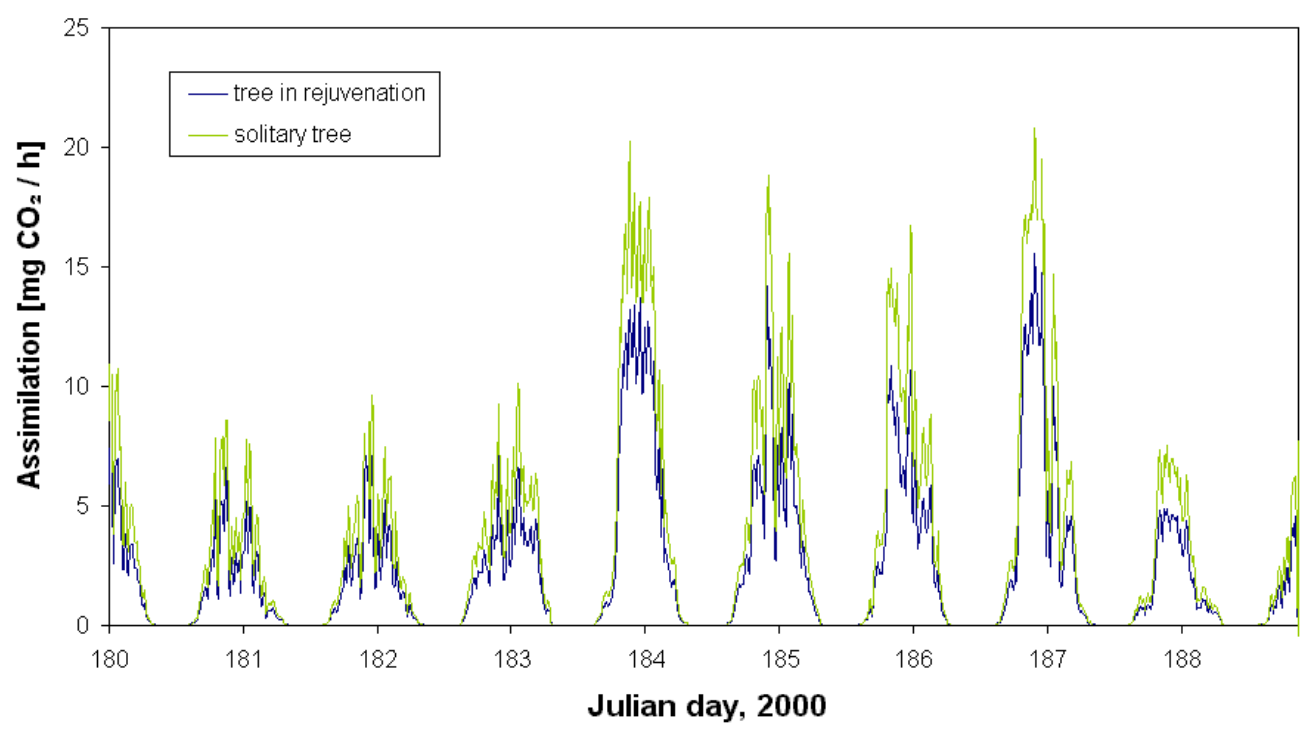

Figure 5-13: The assimilation of the same tree changes drastically between solitary and competition growth.

Through the integration of the models MIR, MuSc and HYDRO in NEXUS, a high degree of automation has been reached, which permits to effectuate the model run in shorter time. The 
user only needs to make some minor modifications, particularly in editing the scenes. NEXUS provides a small tool to automatically generate a scene without terrain, background or other special effects from AMAP.

As with the programs mentioned above, the interconnection with GROGRA required some reprogramming of the original software. With help from J.F. Barci from CIRAD, France, a set of sockets written in $\mathrm{C}$ was added to GROGRA. Kurth rewrote a sensitive L-system developed for spruce (Picea abies; see KURTH, 1999), so that it can accept input from outside. The listing of the L-system is shown in Appendix 2. Figure 5-14 shows three stages of the growth process controlled by this L-system in interaction with NEXUS.
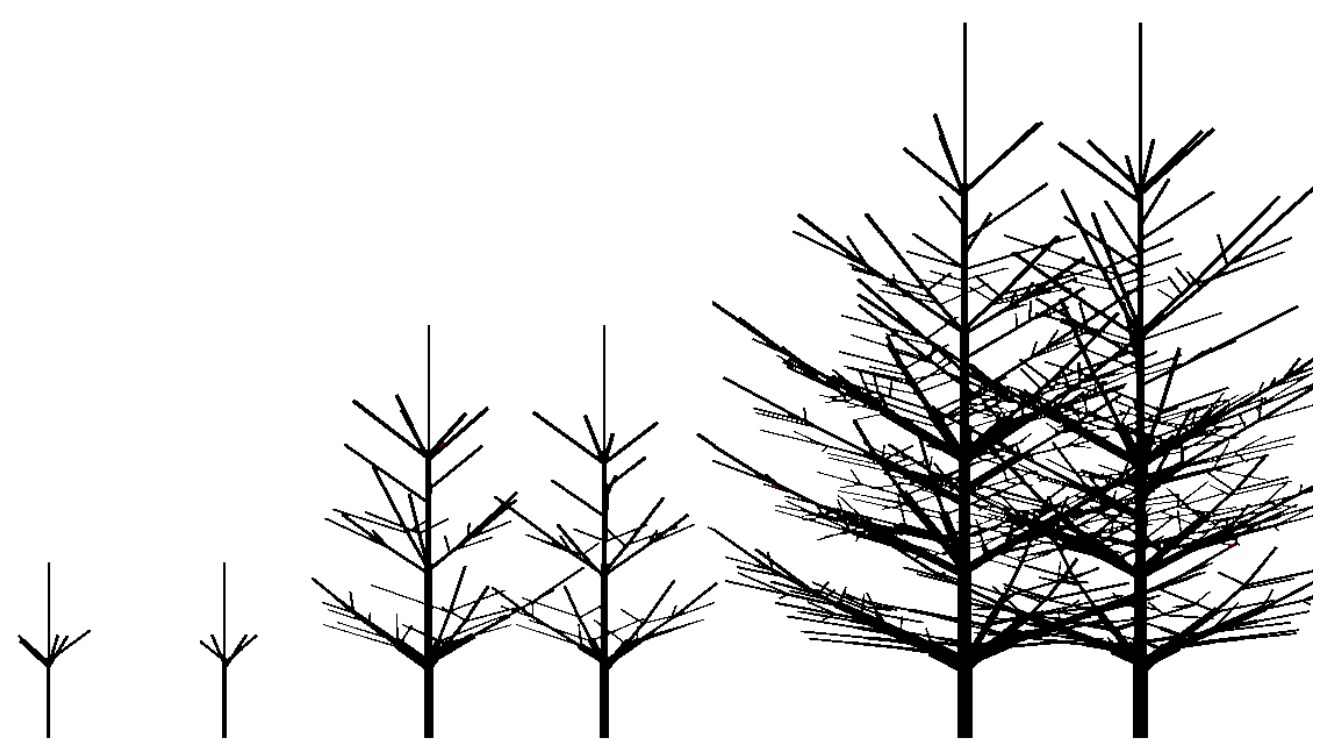

Figure 5-14: Spruce trees generated with the L-system epi2cdi2.ssy, written by Kurth, after 3 years (left), 5 years (middle) and 7 years (right). The L-system reads data from a table which is generated by NEXUS. Flow of data from radiation or hydraulic calculations is not yet realised.

The interconnection with GROGRA works on the basis of a bi-directional data transfer controlled internally by NEXUS. After creating a socket (see 3.3), NEXUS starts GROGRA passing the connection values through the command line. GROGRA then connects itself to this socket. At the beginning of each time step GROGRA waits for a signal from NEXUS to continue the growth simulation, and at the end of the time step it sends a signal back to NEXUS. NEXUS can call other programs to generate the data to be used by GROGRA. In the example, NEXUS creates a simple table of values that are read by GROGRA. These values are then compared with a threshold value within the L-system and different decisions are 
taken as result of this comparison. The values can represent assimilate concentration, hormone concentration, light interception, temperature, etc. In the example, the left tree has a light advantage over the right tree; its shoots are longer, thicker and more numerous than the shoots of the other tree.

Figure 5-15: Scheme of the interconnection NEXUS-GROGRA with a socket for data transfer. GROGRA waits for a signal from NEXUS to start the growth simulation using the generated values, and sends a signal back when the simulation is finished. NEXUS calculates new values using other models like MIR, HYDRO or HYDRA and signalises GROGRA to continue with the growth simulation.

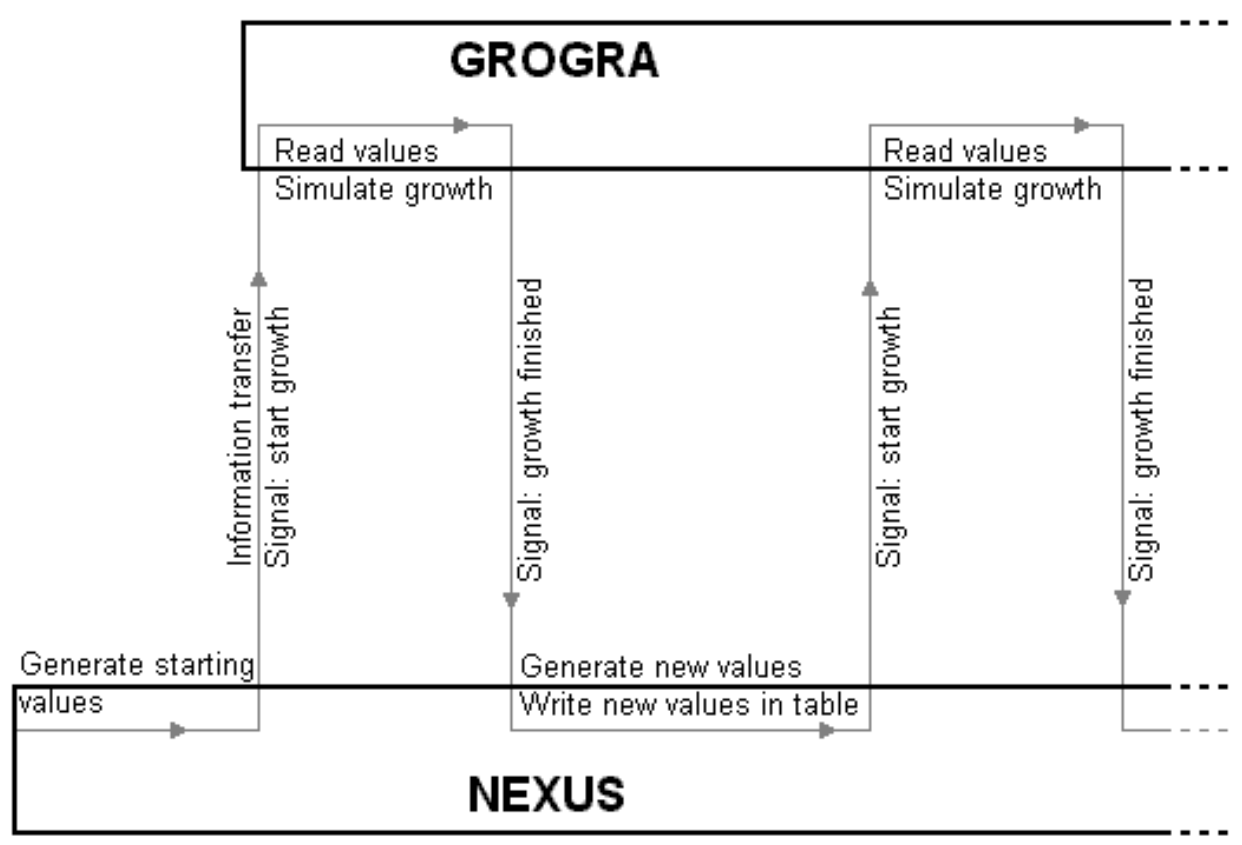

The interconnection with HYDRA is still in work. NEXUS can generate and read the $p b g$ files for HYDRA. However, the simultaneous use of HYDRA and HYDRO to create a more exact hydraulic tool has not yet been implemented. 



\section{Discussion}

The development of complex models for plant growth becomes more important, as the needs of forestry and agriculture grow. Environment changes and the development of modern culture and management methods make it necessary to reconsider old ideas, schemes and models. Sustainable management and ecological forestry build the principles of modern forestry. However, the models used in the praxis till now are no more suitable to explain the changes occurring in our forests and fields. World-wide researchers develop new and accurate models for plant growth or for some other relevant physiological processes.

While in forestry stand oriented models have been principally used as support tools in the management process, individual-oriented models have been developed in biology, agriculture and also in forestry. The development of process and structure models set the basis for modern modelling techniques. With the emergence of modern technologies, high-capacity computers and more accurate measurement tools and methods, also new questions have emerged. The process of modelling itself has developed to a science of its own. With the development of structural-functional models new possibilities to increase the knowledge and understanding of the most basic processes responsible for growth have been provided.

Unfortunately, most of the modelling and research work in this field is still a domain of few specialists. The complexity of models is a cause for the prominent individualism characteristic for most models. In most cases only a few researchers have access to a given model. This way the same model is developed at the same time by different people, possibly without knowing of each other and surely without the possibility of learning mutually from errors and insights. 
With a possibility of sharing knowledge and combining it, the development of more powerful models would be possible. The research process itself would be less ambivalent. There exist many models describing important processes, and others providing a framework for them by describing physical structures. It is not only important to create new and better models, but also to learn if and how they could work together. This thesis is an attempt to do that: Combining and interconnecting process and structure models and even functional-structural models.

The programs and models presented in this thesis, particularly GROGRA, AMAP and LIGNUM are very complex programs that provide a good starting point to begin with the interconnection of procedural and structural models. GROGRA and AMAP provide the framework necessary to build other models, the Growth Engine. The growth engines are not models by themselves but a modelling tool.

The development of single models and their integration occurs often at different scales, both in time and in space. So there arises a question that is often posed. Is it more important to know what happens in one leaf during a few minutes or to know what happens in a forest stand during many years? There is no general answer to this question as it always depends on the point of view. But the integration of models can help to answer this question: both things are important. We intend to know and understand the details to be able to handle better in the whole. In this thesis a series of models were presented that are suitable for the interconnection. Most of these models have been compared often, but each was run in a specific framework, so that the results are not always compatible or usable. The interconnection of models can help us to compare them, because they will now work in a known common environment.

The information transfer is one of the principal problems that must be solved. In this thesis this problem was handled by using common files formats that should become standard for the integration. The idea of the standard format is based on two very generic file formats: MTG from the AMAP Programming Language AML and a structure description format from GROGRA, dtd. 
The development of a generic data interface relies on the acceptance of the proposed file formats and on the utility of the interface. Both formats presented here have assets and drawbacks. The dtd format is more simple but also more limited in the amount of information it can contain. However, due to its simplicity, this format is perfectly suited for the information transfer with many models. Furthermore, GROGRA already provides a wide range of interfaces for other applications, which facilitates the integration process. The MTG format is more complex and it cannot be easily handled by most researchers and programs, but it allows to include more information referring to different spatial scales. Also the increasing importance of this format within the AMAP modelling team makes it essential for future development.

Beyond the acceptance of the formats it is important that the respective researchers are willing and/or able to undertake the modifications needed for the interfaces. This stage of the interconnection is the most critical phase. The interconnection of models developed at different sites and by different workgroups requires a great amount of co-ordination and logistics. The modules presented in this thesis demonstrate how difficult it can be. For the development of the Windows version of GROGRA, the original author, Prof. Dr. Kurth, was always present and ready to answer any questions about the program. Furthermore, he was always prepared to make any changes and expand the original software and to help solve any complications.

For the other two programs developed at the Institute for Forest Biometry and Applied Computer Science of the University of Göttingen the situation was more complicated. The program AIR, which is the predecessor of NEXUS, was developed by Dirk Lanwert. The software HYDRA was developed by Dr. Thomas Früh. Both were co-workers of the Institute that left by the time this project started. The possibilities for communication and feedback were, and still are very constricted. This has made the interconnection with HYDRA, which is being completed by Dr. Michael Schulte, more difficult than originally expected.

The last three modules that were incorporated in the project were MIR, MuSc and HYDRO, which were developed by Dr. Jean Dauzat at the CIRAD in Montpellier, France. He was willing to provide any information about the modules to facilitate the creation of the interface. However, due to the pre-requisites of an object oriented approach, the software had to be 
modified. For different reasons Dr. Dauzat could not make such modifications and he agreed to provide the code so that the programs could be modified. During a stage of three months at the CIRAD, Dr. Dauzat answered any question about the program, as long as it was possible. The collaboration with other programmers of the work team AMAP made it possible to access vital information for understanding the software AMAP and its functionality. It was however often difficult to keep continuous contact between Göttingen and Montpellier so that some questions remained unanswered.

The functionality of the original models presented here has already been tested by the respective authors and other researchers. RAPIDEL (1995) contributed to the validation of MIR, MuSc and HYDRO. He compared the model output with other standard models by calculating the output generated by those models separately. He concludes that it is necessary to integrate the models with the growth simulation programs from AMAP. In this context, a modular model shell would have facilitated the model comparison.

The main objective of this thesis was not to re-validate the models taken, but to demonstrate that this kind of model can (with some effort) be integrated in this project. The parameterisation of the models for oak will be finished by Dr. Schulte in the framework of his project, so that the data from HYDRO can be compared and expanded with the data from HYDRA. However, the basic results obtained here confirm the results from Dauzat and Rapidel for Coffea arabica, and provide an expandable basis for the modelling of oak hydrology and growth.

The development of the NEXUS software is not yet finalised as it will continue beyond the scope of this thesis. The actual version provides some basic tools and the possibility to combine a few programs. A basic collection of sub-models has been created, but the models have not yet been tested within other major models. This includes different formulations for the multiplicative approach of JARVIS (1976) and two wind models.

The software also has some remaining bugs that should be removed later. Some errors are due to the numerics in the programs included. Other bugs were caused by the difference in windows management and event handling on different platforms. 
An important part of the future development of the software is the compatibility with the latest version of AMAP, so that it can work with its Growth Engine. But this depends on the willingness of the managers of the AMAP software to allow insight in the code. This would permit to use NEXUS also with the NT version of AMAP. But this cannot be warranted by the author.

A complete software documentation for users and programmers will be posted on-line in small steps (see section 4). Most of the classes and procedures created will be posted as Open Source with exception of the procedures that handle the actual interface with AMAP. This is protected and Copyright of the CIRAD. 



\section{ZUSAMMENFASSUNG}

Mit den zunehmenden Anforderungen von Forst- und Landwirtschaft nimmt auch die Entwicklung neuer, komplexer Modelle für Pflanzenwachstum an Bedeutung zu. Sowohl Klimaveränderungen als auch die Entwicklung moderner Anbau- und Einrichtungsmethoden machen es notwendig, alte Ideen, Denkschemata und Modelle nachzuprüfen. Nachhaltige und ökologische Waldwirtschaft bilden die Grundlagen der modernen Forstwirtschaft. Die Modelle, die bis heute in der Praxis benutzt werden, sind nicht mehr in der Lage, Veränderungen in unseren Wäldern und Feldern zu erklären. Dies trifft besonders auf Ertragstafeln und andere forstliche Hilfsmittel zu. Weltweit entwickeln Forscher neue und genauere Modelle für Pflanzenstrukturen, -wachstum und andere wichtige physiologische Prozesse. Pflanzenmodelle werden im Allgemeinem aufgeteilt in:

- Prozeßmodelle. Diese Modelle beschenken sich auf die Beschreibung physikalischer, chemischer oder physiologische Prozesse, die mit dem Pflanzenwachstum gekoppelt sind. Beispiele solcher sind Photosynthese, Nährstoff- und Wasseraufnahme, atmosphärischer Transport, Deposition, etc.

- Strukturmodelle. Diese Modelle beschreiben die Struktur und Architektur von Pflanzen und Beständen, sowie die morphologischen Grundprinzipien des Wachstums.

- Struktur-Funktions-Modelle. Diese Art von Modellen wurde in den letzten Jahren immer stärker entwickelt. Es handelt sich um komplexe Modelle, die Prozesse und Strukturen miteinander koppeln. Während in vielen einfacheren Modellen die Struktur nur den räumlichen Rahmen für einen Prozeß darstellt, sind Strukturen in Struktur-Funktions- 
Modellen dynamisch mit den in ihnen ablaufenden Prozessen gekoppelt. Das heißt, die Strukturen ändern sich in Abhängigkeit von den Prozessen.

Während in der Forstwirtschaft Bestandesmodelle allgemein als Hilfsmittel der Forsteinrichtung bis jetzt benutzt werden, sind Einzelpflanzen-Modelle in der Biologie und Landwirtschaft, aber auch zuweilen in der Forstwirtschaft entwickelt worden. Die Entwicklung der Prozeß- und Strukturmodelle bildete die Grundlage für neue Modellansätze. Mit dem Aufkommen moderner Technologien, Hochleistungscomputer und genauerer Meßverfahren und Methoden sind auch neue Fragestellungen aufgetaucht. Der Prozeß der Modellierung hat sich selbst zu einer Wissenschaft entwickelt. Mit der Entwicklung von Struktur-Funktions-Modellen sind neue Möglichkeiten gegeben, das Wissen und Verstehen der grundlegendsten Prozesse, die für das Wachstum verantwortlich sind, zu erweitern.

Leider ist die Modellierungs- und Forschungsarbeit auf diesem Gebiet oft auf wenige Spezialisten beschränkt. Die Komplexität vieler Modelle ist eine der Hauptursachen für den markanten Individualismus, der für die meisten Modelle charakteristisch ist. In den meisten Fällen haben nur einige Wissenschaftler Zugriff auf ein gegebenes Modell. So kann das gleiche Modell gleichzeitig von verschiedenen Leuten entwickelt werden, ohne daß sie voneinander wissen, und ohne die Möglichkeit, von den Fehlern und Erkenntnissen der anderen zu lernen.

Mit der Möglichkeit, Wissen auszutauschen und $\mathrm{zu}$ teilen, würde die Entwicklung leistungsfähigerer Modelle möglich sein. Die Forschung würde eindeutigere Ergebnisse liefern. Es ist nicht nur wichtig, neue und bessere Modelle $\mathrm{zu}$ erstellen, sondern auch zu erkennen, ob und wie sie zusammen arbeiten könnten. Diese Dissertation ist ein Versuch, dies zu tun: Prozeß- und Strukturmodelle, und sogar Struktur-Funktions-Modelle miteinander zu koppeln und zu kombinieren.

Um die Kopplung zu realisieren, wurde ein Programm geschrieben, das den Daten- und Informationsaustausch zwischen verschiedenen Modellen regelt oder gar ermöglicht. Dieses Programm wurde NEXUS genannt. Das Programm wurde in der Programmiersprache $\mathrm{C}++$ unter Anwendung moderner Programmiermethoden geschrieben, insbesondere von Methoden der objektorientierten Programmierung (OOP). 
Objektorientiert bedeutet, daß man Gegenstände, Ideen und Begriffe in abstrakten Strukturen repräsentiert. Diese Strukturen werden Objekte genannt und enthalten alle relevanten Informationen, die das Objekt selbst beschreiben (Attribute), sowie Funktionen, die das Verhalten des Objektes festlegen (Methoden). Diese Objekte können ebenfalls weitere Objekte enthalten. So kann man ein Objekt „Pflanze“ definieren, das aus den Objekten „Stamm“, „Wurzel“ und „Krone“ besteht. Man kann auch Prozesse als Objekte definieren, also z.B. die Objekte „Wasserfluß“, „Transpiration“, „Lichtaufnahme“ und „Photosynthese“. In der objektorientierten Programmierung nennt man die Definition eines Objektes Klasse. Mit Hilfe der Klassen können dann mehrere Objekte vom selben Typ erzeugt werden, sogenannte Instanzen. Also muß man nur einmal die Klasse „Blatt“ definieren, während jedes einzelne Blatt nur eine Instanz dieser Klasse ist.

Der Vorteil der OOP ist die Möglichkeit, Modelle in Module aufzuteilen, die miteinander austauschbar sind. So kann man z.B. in einem Modell für C-Allokation verschiedene Assimilationsmodelle einsetzen und miteinander vergleichen, oder ein Assimilationsmodell wird in verschiedene Allokationsmodelle eingesetzt.

Für die erste Version des Programms wurden verschiedene Module gewählt, die nun miteinander interagieren können. Diese Module behandeln die Strahlungsbilanz, den Wasserhaushalt und das Wachstum. Für die Strahlungsbilanz wurden die Programme MIR (Mapping Incoming Radiation) und MuSc (Multiple Scattering) gewählt. Beide Programme wurden in ihrer ursprünglichen Form von Dr. J. Dauzat am CIRAD (Centre de coopération internationale en recherche agronomique pour le développement), Frankreich, entwickelt. MIR berechnet die direkte Strahlung, die auf jeden Punkt der Pflanze aus verschiedenen Richtungen eintreffen kann. Dafür wird die Raytracing-Methode in Verbindung mit dem Lichthemisphären-Modell von Den Dulk benutzt. Das Modell MuSc berechnet die diffuse Strahlung, die durch Reflexion und Refraktion in den verschiedenen Schichten eines Bestandes entsteht. Für den Wasserfluß im Xylem wurden die Programme HYDRO und HYDRA gewählt. HYDRO wurde ebenfalls von Dr. Dauzat entwickelt. Es berechnet für jedes Blatt anhand meteorologischer Daten Transpiration, Assimilation und Wasserpotentiale. Die Wasserpotentiale werden dazu benutzt, durch ein iteratives Verfahren den Wasserfluß zu berechnen. Das Programm HYDRA wurde von Dr. T. Früh am Institut für Forstliche Biometrie und Informatik der Universität Göttingen entwickelt. Das Modell benutzt ein 
genaueres und numerisch stabileres Verfahren als HYDRO, um den Wasserfluß zu simulieren. Die Transpiration jedoch wird als konstant für alle Assimilationsorgane gesetzt, so daß das Mikroklima nur sehr vergröbert berücksichtigt wird. Als Wachstumsmodule wurden die zwei Programme GROGRA und AMAP gewählt. GROGRA (GROwth GRAmmar interpreter) wurde von Prof. Dr. Kurth am Institut für Forstliche Biometrie und Informatik der Universität Göttingen entwickelt. Es simuliert das Wachstum durch sogenannte L-Systeme (Lindenmayer-Systeme) in festen Zeitschritten. Als Standardschrittweite können Jahre benutzt werden, aber kleinere und größere Abstände sind ebenfalls möglich. AMAP („Atelier de Modélisation de l'Architecture des Plantes“ bzw. „botAnique et bioinforMatique de l'Architecture des Plantes“) wurde am CIRAD von der Arbeitsgruppe von Philippe de Reffye entwickelt. AMAP ist eine Gruppe mächtiger Programme zur Simulation und grafischen Darstellung von Pflanzen und Beständen. Die Simulation des Wachstums wird mit Hilfe der Beschreibung von morphogenetischen Gradienten und unter Verwendung des Begriffs des physiologischen Alters durchgeführt. In Gegensatz zu den anderen Modulen wird AMAP kommerziell benutzt und ist daher nicht unbeschränkt zugänglich.

Alle diese Programme wurden in der Programmiersprache $\mathrm{C}$ geschrieben, ohne OOP zu benutzen. Um die Kopplung der Modelle zu erleichtern, wurden die Module MIR, MuSc und HYDRO reimplementiert und in $\mathrm{C}++$ unter Anwendung von OOP neu geschrieben. GROGRA wurde von Prof. Dr. Kurth modifiziert, um den Datenaustausch zu vereinfachen. HYDRA wird in Rahmen eines parallel laufenden Projekts von Dr. M. Schulte ebenfalls modifiziert.

Als Teil der Arbeit wurde eine Bibliothek von Standardprozeduren und -klassen geschrieben, die anderen Wissenschaftlern zur Verfügung gestellt werden sollen, um die Entwicklung weiterer Module zur erleichtern.

Der Informationsaustausch zwischen den Modulen findet durch den Austausch von Dateien statt. NEXUS ist in der Lage, verschiedene Formate zu lesen und zu schreiben. Für die Darstellung der Pflanzenstruktur wird ein Standardformat angestrebt, um eben den Austausch von Daten zu vereinfachen. Zur Zeit kommen folgende Formate in Frage: 
- Das dtd-Format (descriptive tree data format) von GROGRA. Mit diesem Format lassen sich Baumstrukturen sehr einfach codieren. Außerdem können Zusatzinformationen in Form von Attributen weitergegeben werden.

- Das MTG-Format (Multiscale Tree Graph), entwickelt für die AMAPModellierungssprache AML. Dieses Format ist dem obengenannten ähnlich, aber mit dem Unterschied, daß Informationen in verschiedenen räumlichen Maßstäben dargestellt werden können.

NEXUS ist in der Lage, die verschiedenen Module unter Angabe relevanter Parameter aufzurufen, deren Verhalten zur Laufzeit begrenzt zu steuern, die Ergebnisse zu empfangen und an andere Module weiterzuleiten. Die Kontrolle zur Laufzeit wird mit Hilfe sogenannter „Sockets“ erreicht. Sockets, wie sie hier verwendet werden, sind einfache bidirektionale Datenströme, die zur Weiterleitung von Signalen zwischen zwei oder mehr Programmen dienen. Dies wird in erster Linie dazu benutzt, die Wachstumsmodelle bei jedem Zeitschritt (Wachstumsperiode) so lange anzuhalten, bis die anderen Module Ergebnisse geliefert haben. Es ist möglich, daß verschiedene Module verschiedene Schrittweiten haben, z.B. Jahre für das Wachstum und Tage für die Berechnung der Assimilation. NEXUS hilft dabei, diese miteinander abzustimmen.

Für die Validierung wurden Daten aus Costa Rica und aus Unterlüß, Deutschland, verwendet. Die Daten aus Costa Rica wurden von Dr. Dauzat zur Verfügung gestellt, um die neuen Versionen der Programme MIR, MuSc und HYDRO zu testen. Es wurden meteorologische Daten (Globalstrahlung, PAR, Lufttemperatur, relative Luftfeuchtigkeit und Windgeschwindigkeit über dem Bestand) für einen sonnigen und einen bedeckten Tag im Sommer 1994, sowie Struktur- und Architekturdaten für eine Kaffeepflanze (Coffea arabica) verwendet. In Unterlüß, in der Lüneburger Heide, wurden meteorologische Daten für zehn aufeinanderfolgende Tage im Sommer 2000 gemessen. Außerdem wurde an sieben Eichen (Quercus spc.) Saftfluß gemessen, und die Bäume wurden anschließend kartiert und im dtdFormat codiert.

Unter Verwendung der AMAP-Software wurden virtuelle Szenen hergestellt, in denen die kartierten Bäume und ihre Nachbarn dargestellt werden. Die Szenen wurden benutzt, um die 
Strahlungsbilanz der einzelnen Bäume unter Berücksichtigung ihrer Umwelt zu simulieren. Die Szenen werden von MIR/MuSc benötigt, um mit der Position der verschiedenen Pflanzen die Beschattung und die Lichtreflexion und -refraktion $\mathrm{zu}$ berechnen. Mit diesen Daten berechnet HYDRO die Transpiration und die Assimilation, und anschließend den Wasserfluß. NEXUS liefert die Basisdaten, die HYDRA benötigt, um ebenfalls den Wasserfluß zu berechnen.

Das Verhalten von Transpirations- und Assimilationsraten und des Wasserflusses wurde verglichen für Bäume verschiedener Größe und für dieselben Bäume unter verschiedenen Wuchsbedingungen:

- freistehend gegen Konkurrenz

- $\quad$ sonnige Tage gegen bedeckte Tage

- meteorologische Daten aus Costa Rica gegen Daten aus Deutschland.

Da die Parametrisierung der Modelle für die Baumart Eiche noch nicht abgeschlossen ist, können noch keine quantitativen Aussagen über die Modelle gemacht werden. Trotzdem zeigt die Anwendung der Modelle bei unterschiedlichen Wuchs- und Umweltbedingungen qualitative Unterschiede, die vergleichbar mit denen aus der Literatur sind, indem eine Verringerung der Assimilations- und Transpirationsraten und des Wasserflusses bei geringerem Lichtgenuß und geringerer Temperatur stattfindet. Die simulierten Zeitreihen des Baumwasserflusses stimmen in ihrem qualitativen Verlauf gut mit den gemessenen Zeitreihen überein.

Um die bidirektionale Kopplung mit GROGRA zu testen, wurde ein L-System für die Baumart Fichte (Picea abies (Karst.) L.) von Prof. Dr. Kurth so modifiziert, daß es auf die Signale von NEXUS reagiert. NEXUS generiert Werte in tabellarischer Form, die von GROGRA gelesen werden und mit Schwellenwerten im L-System verglichen werden. Bei Überschreiten dieser Schwellenwerte ändert sich das Verzweigungsverhalten des simulierten 
Baumes. Damit läßt sich eine Rückkopplungsschleife zwischen Bestandes-Mikroklima und Wachstum im Modell repräsentieren.

NEXUS bildet somit einen Grundstein für die Modellkopplung. Die Anpassung von existierenden Modellen an neue Baumarten bleibt eine aufwendige Prozedur, die jedoch durch die Anbindung an andere Modelle zukünftig mehr Früchte tragen kann. 



\section{BibLIOGRAPHY}

AbElson, H.; DiSessa, A.A. (1981): Turtle geometry. M.I.T. Press series in artificial intelligence, Cambridge, $\mathrm{XX}+477 \mathrm{p}$.

ACTON, FORMAN S. (1970): Numerical Methods That Work. New York, Harper and Row.

ADAMS, R.S.; BLACK, T.A.; FLEMING, R.L. (1991): Evapotranspiration and surface conductance in a high elevation, grass-covered forest clear-cut. Agricultural and Forest Meteorology, 56, $173-$ 193.

AMEgLIO, T. (1991): Relations hydriques chez le jeune noyer, de l'échelle de la journée à celle de l'année, en liaison avec quelques aspects de la physiologie de l'arbre. Thèse, Université B. Pascal, Clermont-Ferrand.

ANDERSON, M.C. (1966): Stand structure and light penetration. II. Theoretical analysis. Journal of Applied Ecology, 3, 41-54.

Anzola, Gustavo (1998): Auswertung und Modellierung der Morphologie junger Fichten (Picea abies (L.) Karst.) in Abhängigkeit von Licht und Wuchsdichte. Diplomarbeit an der Fakultät für Forstwissenschaften und Waldökologie der Universität Göttingen.

Barczi, J.-F.; DE RefFye, Ph.; CARAglio, Y. (1997): Essai sur l'identification et la mise en œuvre des paramètres nécessaires à la simulation d'une architecture végétale. Le logiciel AMAPsim. In: Bouchon J., Reffye P. de, Barthélémy D. (Eds.), Modélisation et Simulation de l'Architecture des Végétaux, INRA, Paris, 205-254.

Balandier, Ph.; Lacointe, A.; Le Roux, X.; Sinoquet, H.; Cruiziat, P.; Le Dizès, S. (2000): SIMWAL: A structural-functional model simulating single walnut tree growth in response to climate and pruning. Annals of Forest Science, 57, 571-585.

BALL, J.T.; WOODROW, L.E.; BERRY, J.A. (1987): A model predicting stomatal conductance and its contribution to the control of photosynthesis under different environmental conditions. In: J. Biggins (Ed.) Progress in photosynthesis research. Martinus Nijhoff, Dordrecht, 221-224.

BARTÁK, M.; JANOUŠ, D. (1989): Crown structure of dominant Turkey oak (Quercus cerris L.) in an oak-hornbeam forest stand. Ekológia, USSR, 8(2), 123-130.

BARTh, R.; BeIER, E.; PAHNKe, B. (1996), Grafikprogrammierung mit OpenGL. Addison-Wesley, Bonn, Paris, 240 p. 
BASSOW, S.L.; FORD, E.D.; KIESTER, A.R. (1990): A critique of carbon-based tree growth models. In: R.K. Dixon; R.S. Meldahl; G.A. Ruark and W.G. Warren (Eds.), Process modeling of forest growth responses to environmental stress, Portland, 50-57.

BAyrhuber, Horst; Kull, Ulrich; BäßLer, Ulrich; DANZer, Albert (1991): Linder Biologie. Lehrbuch für die Oberstufe. J.B. Metzlersche Verlagsbuchhandlung, Stuttgart, $528 \mathrm{~S}$.

Berninger, F.; NikinMaA, E.; SiEvÄNEn R.; NYGRen, P. (2000): Modelling of reserve carbohydrate dynamics, regrowth and nodulation in a $\mathrm{N}_{2}$-fixing tree managed by periodic prunings. Plant, Cell and Environment, 23, 1025-1040.

BIDEL, LUC (1994): Développement d'un modèle numérique de simulation de la transpiration et la circulation de la sève brute dans l'appareil végétatif d'une plante ligneuse. Mémoire de fin d'études, Ecole National Supérieure Agronomique de Rennes.

BLAISE, F. (1991): Simulation du parallélisme dans la croissance des plantes et applications. Thèse, Université Lois Pasteur, Strasbourg, France.

BLENDINGER, C. (1995) silVlow-ein Programm zur Berechnung gesättigt-ungesättigter Strömung im zweidimensionalen porösen Medium. Schriftenreihe SFB 350, Universität Bonn,

BÖHM, W. (1979): Methods of Studying Root Systems, Springer, Berlin, Heidelberg, New York.

Bosc, AleXANDRe (2000): EMILION, a tree functional-structural model: Presentation and first application to the analysis of branch carbon balance. Annals of Forest Science, Nr. 35, 555569.

Bossel, HARTMUT (1994): TREEDYN3 Forest Simulation Model. Mathematical model, program documentation, and simulation results. Berichte des Forschungszentrums Waldökosysteme, Reihe B, Band 35, Göttingen.

BRECKLING, B. (1994): An individual based model for the study of pattern and process in plant ecology: An application of object oriented programming. Projektzentrum Ökosystemforschung. Universität Kiel. ECOSYS, 4, 241-254.

Brough, D.W.; JONES, H.G.; GraCE, J. (1986): Diurnal changes in water content of the stems of apple trees, as influenced by irrigation. Plant Cell Environment, 9, 1-7.

BRUNET, Y. (1997): Modélisation architecturale et transferts turbulents. In: B. Andrieu (Ed.), Modélisation Architecturale, Actes du Séminaire, INRA-Bioclimatologie, Paris, 231-233.

BRUnet, Y.; Finnigan, J.J.; RAUPACH, M.R. (1994): A wind tunnel study of air flow in waving wheat: single-point velocity statistics. Boundary Layer Meteorology, 70, 96-132.

Brunet, Y.; LAVILle, P.; Flure, D.; Baculatat, T. B. (1996): La mesure des flux turbulents. In: "De la plante au couvert végétal", Cruiziat P., Lagouarde J.P. (eds.), Ecole-Chercheurs INRA en Bioclimatologie, Le Croisic, 03-07/04/1995, INRA Département de Bioclimatologie, tome $1,441-459$.

ČERMÁK, J.; DemL, M.; PenKa, M. (1973): A new method of sap flow rate determination in trees. Biologia Plantarum, Praha, 13, 3, 171-178.

ČERMÁK, J.; DEML, M.; PENKA, M. (1976): Improvement of the method of sap flow rate determination in full-grown trees based on heat balance with direct electric heating of xylem. Biologia Plantarum, Praha, 18, 105-110. 
Chambers, J.L.; Hinckley, T.M.; CoX, G.S.; MetCalf, C.L.; Aslin, R.G. (1985): Boundary-line analysis and modes of leaf conductance for oak-hickory forest species. Forest Science, 31, 2, 431-450.

ChibA, N.; OhKawa, S.; Muraoka, K.; MiURA, M. (1994): Visual simulation of botanical trees based on virtual heliotropism and dormancy break. The Journal of Visualization and Computer Animation, 5, 3-15.

CHIBA, YUKIHIRO (1990): Plant form analysis based on the pipe model theory - I. A statistical model within the crown. Ecological Research, 5, 207-220.

CHIBA, YUKIHIRO (1991): Plant form analysis based on the pipe model theory - I. Quantitative analysis of ramification in morphology. Ecological Research, 6, 21-28.

ChibA, YUKIHIRo (2000): Mathematical Model of Stem Formation and Tree Architectural Development. Plant Biomechanics, 606-612.

CHIBA, YUKIHIRO; SHINOZAKI, KICHIRO (1994): A simple mathematical model of growth pattern in tree stems. Annals of Botany, 73, 91-98.

Chiba, Yukiniro; FuJimori, TAKaO; KiYono, YoshiYUKi (1988): Another Interpretation of the Profile Diagram and Its Availability with Consideration of the Growth Process of Forest Trees. Journal of the Japan Forest Society. 70 (6), 245-254.

CHIEN, T.W.; JÜRGENSEN, H. (1992): Parameterized L systems for modelling: Potential and limitations. In: G. Rosenberg and A. Salomaa. (Eds.), Lindenmayer Systems, Springer Verlag, Berlin, 213-229.

Choudhury, B.L.; MonTeith, J.L. (1988): A four-layer model for heat budget of homogeneous land surfaces. Q. J. R. Meteorol. Society, 114, 373-398.

ClausnitZer, V.; HoPMANS, J.W. (1994): Simultaneous modeling of transient three-dimensional root growth and soil water flow. Plant and Soil, 164, 299-314.

CONERS, HEINZ (2001): Wasseraufnahme und artspezifische hydraulische Eigenschaften der Feinwurzeln von Buche, Eiche und Fichte: In situ-Messungen an Altbäumen. Dissertation, Universität Göttingen.

COnStANTIN, J. (1993): Stoffeinträge in ein Fichtenwaldökosystem durch Deposition luftgetragener Partikel und Nebeltröpfchen. Berichte des Forschungszentrums Waldökosysteme, Reihe A, Bd.106, Göttingen.

COWAN, I.R.; FARQUHAR, G.D. (1977): Stomatal function in relation to leaf metabolism and environment. Symp. Soc. Exp. Biol., 31, 471-505.

Cruiziat, P.; Granier, A.; Claustres, J.P.; Lachaize, D. (1989): Diurnal evolution of water flow in an individual spruce: experimental and theoretical study. Annales des Sciences Forestières, $353-356$.

Cruiziat, P.; Ameglio, T.; Cochard, H. (2001): La cavitation: un mécanisme perturbant la circulation de l'eau chez les végétaux. Mécanique \& Industries, Vol.2, 4, 289-298.

DAUdeT, F.-A.; LE RouX, X.; SinOQuet, H.; ADAM, B. (1999): Wind speed and leaf boundary layer conductance variations within tree crown: consequences on leaf-to-atmosphere coupling and tree function. Agricultural and Forest Meteorology, 97, 171-185. 
DAUZAT, J. (1993): Simulated plants and radiative transfer simulation. In: C. Varlet-Grancher, R. Bonhomme, H. Sinoquet (Eds.) Crop Structure and Light Microclimate, Paris, 271-278.

DAUZAT, J. (1994): Simulation des échanges radiatifs sur maquettes informatiques de Elais guineensis. Oléagineux, 49 (3), 81-90.

DAUZAT, J.; EROY, M.N. (1997). Simulating light regime and intercrop yields in coconut based farming systems. European Journal of Agronomy 7, 63-74.

DAUZAT, J.; HAUTECOEUR, O. (1991): Simulation des transferts radiatifs sur maquettes informatiques de couverts végétaux. In: Physical measurements and signatures in remote sensing, Proc. $5^{\text {th }}$ Int. Coll. ESA, ESA, Frascatti, Italy, 415-418.

DAUZAT, J.; RAPIDEL, B. (1998). Modélisation du fonctionnement hydrique des plantes. Application au calcul de la transpiration du caféier. In: Architecture et modélisation en arboriculture fruitière, 11ème colloque sur les recherches fruitières, Montpellier, France 5-6/03/1998. INRA-Ctifl; pp. 253-262.- [A-249].

De CAStro, F.; Fetcher, N. (1998): Three dimensional model of the interception of light by a canopy. Agricultural and Forest Meteorology, 90, 215-233.

Deleuze, C.; Hervé, J.-C.; Colin, F.; Ribeyrolles, L. (1996): Modelling crown shape of Picea abies: spacing effects. Canadian Journal of Forest Research, 26, 1957-1966.

DEN DULK, J.A. (1989): The interpretation of remote sensing, a feasibility study. Doctoral Thesis, Wageningen.

DE REFFYe, P.; DinOUARD, P.; BARTHELEMY, D. (1991): Modélisation et simulation de l'architecture de l'orme du Japon Zelkova serrata (Thunb.) Makino (Ulmaceae): La notion d'axe de référence. In: C. Edelin (Ed.), L'arbre. Biologie et développement. Naturalia Monspeliensia, 251-266.

DE RefFye, P.; Houllier, F.; Blaise, F.; BARThÉlÉmy, D.; DAuzat, J.; Auclair, D. (1995a): A model simulating above- and below- ground tree architecture with agroforestry applications. In: Agroforestry: Science; Policy and Practice, 20th IUFRO World Congress, F.L. Sinclair (Ed.), Tampere, Finland 06-12/08/1995. Agroforestry Systems, Vol. 30; pp. 175-197.

De RefFye, P.; Houllier, F.; Blaise, F.; Barthelemy, D.; Dauzat, J.;Auclair, D. (1995b): Modélisation et simulation de la croissance d'une architecture végétale: une approche morphogénétique expérimentale. Tendances nouvelles en modélisation pour l'environnement, Actes des Journées du Programme Environnement, Vie et Sociétés, Session B Modélisation des systèmes complexes, nouveaux modèles, validation de modèles; Cité des Sciences et de l'Industrie; Paris, France 15-17/01/1996. CNRS Ed.; 91-112.

De RefFye, P.; Fourcaud, T.; Blaise, F.; Barthélémy, D.; Houllier, F. (1997): A functional model of tree growth and tree architecture. Silva Fennica, 31, 297-311.

DORUSKA, P.F.; BURKHARD, H.E. (1994): Modeling the diameter and locational distribution of branches within the crowns of loblolly pine trees in unthinned plantations. Canadian Journal of Forest Research, 24, 2363-2376.

DZIERZON, H.; KURTH, W. (2002): LIGNUM: A Finnish tree growth model and its interface to the French AML-Database. Proceedings of the 8th Workshop on Individual-based Models and Structural-Functional Models, Helenenau (10.-12. 7. 2000), Peter Lang Verlag, Frankfurt a. M. (in press). 
EDWARDS, W.R.N.; JARVIS, P.G. (1982) Relation between water content, potential and permeability in stems of conifers. Plant Cell Environment, 5, 271-277

EdWARDS, W.R.N.; JARVIS, P.G.; LANDSBerG, J.J.; TAlbot, H. (1986): A dynamic model for studying flow of water in single trees. Tree Physiology, 1, 309-324

EsChENBACH, Christiane (2000): The effect of light acclimation of single leaves on whole tree growth and competition - an application of tree growth model ALMIS. Annals of Forest Science 57, 599-609.

Falge, E.; Graber, W.; Siegwolf, R; Tenhunen, J.D. (1996): A model of the gas exchange response of Picea abies to habitat conditions. Trees, 10, 277-287.

FAlge, E.; RYel, R.J.; Alsheimer, M; TEnhunen, J.D. (1997): Effects of stand structure and physiology on forest gas exchange: a simulation study for Norway spruce. Trees, 11, 436-448.

FARNSWORTH, K.D.; VAN GARDINGEN, P.R. (1995): Allometric analysis of Sitka spruce branches: mechanical versus hydraulic design principles. Trees, 10, 1-12.

FARQUHAR, G.D. (1978): Feedforward responses of stomata to humidity. Australian Journal of Plant Physiology, 5, 787-800.

FARQUHAR, G.D.; WONG, S.C. (1984): An empirical model of stomatal conductance. Aust. Journal of Plant Physiology, 11, 191-210.

FARQUHAR, G.D.; VON CAEMMERER, S.; BERRY, J.A. (1980): A biochemical model of photosynthetic $\mathrm{CO}_{2}$ assimilation in leaves of $\mathrm{C}_{3}$ species. Planta, 149, 78-90.

FEDERER, C.A. (1980): Paper birch and white oak saplings differ in response to drought. Forest Science, 26, 313-324.

FINNIGAN, J.J.; RAUPACH, M.R. (1986): Transfer processes in plant canopies in relation to stomatal characteristics. In: Zeiger; Farquhar; Cowan (Eds.) Stomata Function, Stanford University Press, 385-429.

FRÜH, T. (1995): Entwicklung eines Simulationsmodells zur Untersuchung des Wasserflusses in der verzweigten Baumarchitektur. Berichte des Forschungszentrums Waldökosysteme Göttingen, Band 131.

FRÜH, T.; KURTH, W. (1999): The Hydraulic System of Trees: Theoretical Framework and Numerical Simulation. Journal of Theoretical Biology, 201, 251-270.

GAFFREY, D.; KNIEMEYER, O. (2001): The elasto-mechanical behavior of Douglas fir, its sensitivity to tree-specific properties, wind and snow loads, and implications for stability - a simulation study. Submitted.

GAFFrey, D.; SlobodA, B. (2001): Tree mechanics, hydraulics and needle-mass distribution as a possible basis for explaining the dynamics of stem morphology. Journal of Forest Science, 47 (6), 241-254.

GoDIN, C. (2000): Representing and encoding plant architecture: A review. Annals of Forest Science, $57,413-438$.

Godin, C.; CARAgLio, Y. (1998): A multiscale model of plant topological structures. Journal of Theoretical Biology, 191, 1-46. 
Godin, C.; Costes, E.; SinOQueT, H. (1999a): A method for describing plant architecture which integrates topology and geometry. Annals of Botany, 84, 343-357.

Godin, C.; GuÉDON, Y.; COSTES, E. (1999b): Exploration of a plant architecture database with the AMAPmod software illustrated on an apple tree hybrid family. Agronomie 19, 163-184, Paris.

GOUdRIAAN, J. (1977): Crop micrometeorology: a simulation study. Simulation Monographs, Wageningen, $249 \mathrm{p}$.

Goudriann, J. (1982): Potential production processes. In: F.W. Penning de Vries; H.H. van Laar (Eds.), Simulation Monographs, Wageningen, 99-113.

Gruber, F. (1987): Das Verzweigungssystem und der Nadelfall der Fichte (Picea abies (L.) Karst.) als Grundlage zur Beurteilung von Waldschäden. Berichte des Forschungszentrums Waldökosysteme Göttingen, Reihe A, Band 26, Göttingen.

GuEDON, Y. (1997): Modélisation de séquences, d'événements décrivant la mise en place d'éléments botaniques. In: J. Bouchon, Ph. De Reffye, D. Barthélémy (Eds.), Modélisation et Simulation de l'Architecture des Végétaux, INRA, Paris, 187-202.

HÄCKEL, H. (1999): Meteorologie. UTB für Wissenschaft, Ulmer, Stuttgart, 448 p.

HAINZL, J. (1981): Mathematik für Naturwissenschaftler, Teubner Studienbücher, Stuttgart.

HALLÉ, F. (1986): Modular growth in seed plants. Phil. Trans. Royal Society London, B, 313, 77-87.

Halle, F.; Oldeman, R.A.A. (1970): Essai sur l'architecture et la dynamique de croissance des arbres tropicaux. Masson, Paris.

Hallé, F.; Oldeman, R.A.A.; Tomlinson, P.B. (1978): Tropical Trees and Forests. Springer, Berlin.

HERMAN, G.T.; SCHIFF, G.L. (1975): Simulation of multi-gradient models of organisms in the context of L-systems. Journal of Theoretical Biology, 54, 35-46.

HoRnUnG, U.; Messing, W. (1984): Poröse Medien und Simulation. Kirchzarten, Verlag Beiträge zur Hydrologie.

Host, G.E.; RAUSCHER, M.; ISEBRANDS, J.G.; MiChaEL, D.A. (1990): Validation of photosynthate in production in ECOPHYS, an ecophysiological growth process model of Populus. Tree Physiology, 7, 283-269.

IBROM, A. (1993): Die Deposition und Pflanzenauswaschung (Leaching) von Pflanzenstoffen in einem Fichtenbestand im Solling. Forschungszentrum Waldökosysteme der Universität Göttingen.

ISTOK, J.D. (1989): Groundwater Modeling by the Finite Element Method, Washington, DC, American Geophysical Union.

Jallas, E.; Martin, P.; Sequeira, R.; Turnar, S.; Cretenet, M.; GérardeauX, E. (2000): Virtual COTONS ${ }^{\circledR}$, the Firstborn of the Next Generation of Simulation Model. In: J.-C. Heudin (Ed.), Virtual Worlds 2000, Springer, Berlin, 235-244.

JARVIS, P.G. (1976): The interpretation of the variations in leaf water potential and stomatal conductance found in canopies in the field. Phil. Trans. R. Soc. London, B. 273, 593-610. 
JARVIS, P.G.; MCNAUGHTON, K.G. (1986): Stomatal control of transpiration: scaling up from leaf to region. Adv. In Ecology Research, 15, 1-49.

JARVIS, P.G.; EDWARDS, W.R.N.; TALbOT, H. (1981): Model of plant and crop use. In: D.A. Rose, A.C. Edwards (Eds.), Mathematics and plant physiology. Experimental Botany, 16.

JONES, H.G. (1992): Plants and microclimate, a quantitative approach to environmental plant physiology. Cambridge University Press, $452 \mathrm{p}$.

KAHN, M.; PRETZSCH, H. (1997): Das Wuchsmodell SILVA - Parametrisierung der Version 2.1 für Rein- und Mischbestände aus Fichte und Buche. Allgemeine Forst- und Jagdzeitung, 168 (67), 115-123.

KELLOMÄKI, S.; IKONEN, V.P. (1996): Light and structural development of Scots pine crown. The Helsinki workshop on functional-structural tree models (12-13/12/1996). Reader with abstracts. METLA, Helsinki.

KellomÄKI, S.; KURTTIO; O. (1991): A model for the structural development of a Scot pine crown based on modular growth. Forest Ecology and Management, 43, 103-123.

KellomÄKI, S.; Strandman, H. (1995): A model for the structural growth of young Scots pine crowns based on light interception by shoots. Ecological Modelling 80, 237-250.

KIESTER, R.; Ford, E.D.; Ford, R.; GAY, C.A.; Avery, A.; Droessler, T.D.; Bassow, S. (1990): NAPAP Report 17: Development and Use of Tree and Forest Response Models. Chapter: Simple Whole Tree. Acidic Deposition: State of Science and Technology, Vol. III. Washington, D.C., 35-127.

KnyaZhiKin, Y.; KranigK, J.; Miessen, G.; PANFyorov, O.; Gravenhorst, G. (1996): Modelling three-dimensional distribution of photosynthetically active radiation in sloping coniferous stands. Biomass and Bioenergy, 11, 189-200.

KozlowsKi, T.T.; KRAMER, P.J.; PALladY; S.G. (1991): The physiological ecology of woody plants. Academic Press, San Diego, 567 p.

KRAMER, P.J. (1988): Changing concepts regarding plant water relations. Plant Cell and Environment, $14,531-544$.

KranigK, J. (1995): Ein Modell für den Strahlungstransport in Fichtenbeständen. Dissertation, Universität Göttingen.

KURTH, W. (1994): Growth Grammar Interpreter GROGRA 2.4. A software tool for the 3dimensional interpretation of stochastic, sensitive growth grammars in the context of plant modelling. Introduction and Reference Manual. Berichte des Forschungszentrums Waldökosysteme, Reihe B, Band 38, Göttingen.

KURTH, W. (1997): Computersimulation von Baumarchitektur und -wachstum. Dreidimensionale Modelle in der Forstwissenschaft. Beiträge für Forstwirtschaft und Landschaftsökölogie 31, 1, S. 22-26.

KURTH, W. (1999): Die Simulation der Baumarchitektur mit Wachtumsgrammatiken. Stochastische, sensitive L-Systeme als formale Basis für dynamische, morphologische Modelle der Verzweigungsstruktur von Gehölzen. Wissenschaftlicher Verlag Berlin. 
KURTH, W. (2002): Spatial structure, sensitivity and communication in rule-based models. In: Proceedings of the 8th Workshop on Individual-based Models and Structural-Functional Models, Helenenau (10.-12. 7. 2000), Peter Lang Verlag, Frankfurt a. M. (in press).

Kurth, W.; Anzola JÜrgenson, G. (1997): Triebwachstum und Verzweigung junger Fichten in Abhängigkeit von den beiden Einflußgrößen „Beschattung“ und „Wuchsdichte“: Datenaufbereitung und -analyse mit GROGRA. Deutscher Verband Forstlicher Forschungsanstalten, Sektion Forstliche Biometrie und Informatik, 10. Tagung, Freiburg i. Br., 24.-26. 9. 1997, Hg.: D. Pelz, Biotechn. Fakultät Univ. Ljubljana, 89-108.

KURTH, W.; FRÜH, T. (1998): Modelling crown structure and hydraulic function in conifers I. A stochastic growth grammar for the developing branching system of spruce. (submited).

KURTH, W.; LANWERT, D. (1995): Biometrische Grundlagen für ein dynamisches Architekturmodell der Fichte (Picea abies (L.) Karst.). Allgemeine Forst- und Jagdzeitung 166 (9-10), S. 177184.

KURTH, W.; SLOBODA, B. (1997): Growth grammars simulating trees - an extension of L-systems incorporating local variables and sensitivity. Silva Fennica, 31 (3), 285-295.

Kurth, W.; SlobodA, B. (2001): Sensitive Growth Grammars Specifying Models of Forest Structure, Competition and Plant-Herbivore Interaction. Proceedings of the IUFRO 4.11 Congress "Forest Biometry, Modelling and Information Science", Greenwich, UK, June $25^{\text {th }}$ $29^{\text {th }} 2001$.

KuUluvainen, T.; PukKala, T. (1987): Effect of crown shape and tree distribution on the spatial distribution of shade. Agricultural and Forest Meteorology, 40, 215-231.

LACOINTE, A. (2000): Carbon allocation among tree organs: A review of basic processes and representation in functional-structural tree models. Annals of Forest Science, 57, 521-533.

LAFOLIE, F.; BRUCKLER, L.; TARDIEU, F. (1991): Modeling root water potential and soil-root water transport: I. Model presentation. Soil Sci. Soc. Am. J., 55, 1203-1212.

LANWERT, D. (1997): Water use of forest trees: Implementation of a drought-stress reaction predictor for tree and stand growth, based on combining well-tried botanical and physical models. Final report of the project AIR3-BM94-4013, CIRAD, Montpellier, France.

LANWERT, D.; DAUZAT, J.; FRÜH, TH. (1998): Water use of forest trees: a possibility of combining structures and functional models. Bayreuther Forum Ökologie, 52, 117-128.

LeCOUSTRE, R. (1990): Simulation des transferts radiatifs sur maquettes informatiques d'Elaeis guineensis. ATP No. 222 / IRHO, Modélisation des transferts radiatifs au sein d'une palmeraie, Document No. 2, CIRAD, Montpellier, France.

LeCoustre, R.; De RefFye, PH. (1993): AMAP, un modeleur de végétaux, un ensemble de logiciels de $\mathrm{CAO} / \mathrm{DAO}$ à l'usage de professionnels de l'aménagement et des paysages. Revue Horticole Suisse 66, 142-146.

LEDIG, S. (1991): Représentation graphique d'épicéas. INRA, Centre de Nancy, Recherche Forestière. Mention Complémentaire en Informatique pour l'Instrumentation Scientifique et Médicale. Année 1990-1991.

LEHNER, B. (2001): KDE- und Qt-Programmierung. Addison-Wesley, München, Boston, 779 p. 
Le Roux, X.; Grand, S.; Dreyer, E.; Daudet, F.A. (1999): Parameterization and testing of a biochemically based photosynthesis model for walnut (Juglans regia) trees and seedlings. Tree Physiology, 19, 481-492.

LEWANDOWSKI, A.; VON GADOW, K. (1996): Ein heuristischer Ansatz zur Reproduktion von Waldbeständen. Allgemeine Forst- und Jagdzeitung, 168. Jg., 9, 170-174.

LHOMME, J.P.; MONTENY, B.; AMADOU, M. (1994): Estimating sensible heat flux from radiometric temperature over sparse millet. Agricultural and Forest Meteorology, 68, 77-91.

LI, J.C.R. (1966): Numerical Mathematics, Edwards Brothers, Inc., Michigan, 643 p.

LINDENMAYER, A. (1968): Mathematical models for cellular interactions in development. I. Filaments with one-sided inputs. Journal of Theoretical Biology, 18, 280-299.

LINDENMAYER, A. (1974): Adding continuous components to L-Systems. In: G. Rosenberg and A. Salomaa (Eds.), L-Systems, Lecture Notes in Computer Science, 15, 53-58.

LipPMAN, S.B.; LAJOIE, J. (1998): C++ Primer. Addison-Wesley, Reading, Massachusetts, 1236 p.

LIST, RALF; KÜPPERS, MANFRED (1998): Light climate and assimilate distribution within segment oriented woody plants growth in the simulation program MADEIRA. In: A. Kastner-Maresch, W. Kurth, M. Sonntag, B. Breckling (Eds.) Individual-based structural and functional models in ecology, Bayreuther Forum Ökologie, Band 52, 141-153.

LIST, R.; KÜPPERS, M.; SCHRÖDER, F. (1994): Madeira - ein Programm zur Simulation des Wachstums (Biomasse und Architektur) von Holzgewächsen. Verhandlungen der Gesellschaft für Ökologie, 23, 39-48.

LIVINGSTON, N.J.; BLACK, T.A. (1987): Stomatal characteristics and transpiration of three species of conifer seedlings planted on a high elevation south-facing clear-cut. Canadian Journal of Forest Research, 17, 1273-1282.

LOUP, C.; FOURNIER, M.; CHANSON, B. (1991): Relations entre architecture, mécanique et anatomie de l'arbre. Cas d'un pin maritime (Pinus pinaster Soland.). In: C. Edelin (Ed.) L'ARBRE. Biologie et Développement. Naturalia Monspeliensia, H.S., Montpellier, 181-195.

LÜCK, J.; LÜCK, H.B.; BAKKALI, M. (1990): A comprehensive model for acrotonic, mesotonic and basitonic branching in plants. Acta Biotheoretica, 38, 257-288.

Maillette, L. (1990): The value of meristem states, as estimated by a discrete-time Markov chain. Oikos, 59, 245-240.

MÄKELÄ, A. (1986): Implications of the pipe model theory on dry matter partitioning and height growth in trees. Journal of Theoretical Biology, 123, 103-120.

Mattheck, C. (1993): Design in der Natur. Der Baum als Lehrmeister. Rombach Ökologie, Freiburg.

MCMahon, T.A.; KRONAUER, R.E. (1976): Tree structures: Deducing the principle of mechanical design. Journal of Theoretical Biology, 59, 443-466.

MCNAUGHTON, K.G.; JARVIS, P.G. (1983): Predicting effects of vegetation changes on transpiration and evaporation. In: T.T. Kozlowski (Ed.), Water Deficits and Plant Growth, Vol. VII, Academic Press, 1-47. 
MĚCH, R; PRUSINKIEWICZ, P. (1996): Visual models of plants interacting with their environments. Computer Graphics Proceedings, Annual Conference Series, SIGGRAPH96, New Orleans (August 1996), pp. 397-410.

MenCUCCINI, M.; GRACE, J.; FioRAVANTI, M. (1997): Biomechanical and hydraulic determinants of tree structure in Scots pine: anatomical characteristics. Tree Physiology, 17, 105-113.

MonTEITH, J.L. (1965): Principles of environmental physics. Arnold, London, 236 p.

MoOn, P.; SPEnCER, D.E. (1942): Illumination from a non-uniform sky. Trans. Illum. Eng. Soc., 37, $707-712$.

MoRgenstern, M. (1996): Die Gleichungen für wassergesättigten und -ungesättigten Fluß im Untergrund. In: Dokumentation von Wassehaushaltsmodellen. Hörmann, G., Schmidt, J. (eds.). Berichte des Forschungszentrums Waldökosysteme, Reihe B, Bd. 42, 1-11, Göttingen.

MÖHRING, B. (1980): Über die Zusammenhänge zwischen Baumform und Schneebruchanfälligkeit bei der Fichte. Diplomarbeit, Georg-August-Universität Göttingen.

MurRAY, D.M.; VON GADOW, K. (1993): A Flexible Yield Model for Regional Timber Forecasting. South. Journal of Applied Forestry, 17(2), 112-115

NeILSON, R.E.; JARVIS, P.G. (1975): Photosynthesis in Sitka spruce (Picea sitchensis (Bong.) Carr.). VI. Response of stomata to temperature. Journal of Applied Ecology, 12, 879-891.

NIEDERSÄCHSISCHE LANDESREGIERUNG (1992): Niedersächsisches Programm zur langfristigen ökologischen Waldentwicklung in den Landesforsten, Hannover.

NISHIDA, T. (1980): K0L-system simulating almost but not exactly the same development. Journal of Theoretical Biology, 152, 429-452.

NÜTZMANN, E. (1999): Modellierung des turbulenten Austausches zwischen der Prandtl-Schicht und der Waldatmosphäre. Dissertation, Georg-August-Universität Göttingen.

OpPelt, A.; KURTH, W.; GODBOLD; D.L. (2001): Topology, scaling relations and Leonardo's rule in root systems from African tree species. Tree Physiology, 21, 117-128.

OTTO, HANS-JÜRGEN (1989): Aus dem Walde. Langfristige, ökologische Waldbauplanung für die Niedersächsischen Landesforsten - Band 1, Mitteilungen aus der Niedersächsischen Landesverwaltung, Heft 42, $442 \mathrm{~S}$.

OTTO, HANS-JÜRGEN (1991): Aus dem Walde. Langfristige, ökologische Waldbauplanung für die Niedersächsischen Landesforsten - Band 2, Mitteilungen aus der Niedersächsischen Landesverwaltung, Heft 43, 527 S.

PAGÈS, L. (2000): How to include organ interactions in models of the root system architecture? The concept of endogenous environment. Annals of Forest Science, 57, 535-541.

Penman, H.L. (1948): Natural evaporation from open water, bare soil and grass. Proc. Royal Society, London, A 194, 120-145.

Perttunen, J.; Sievänen, R.; NikinmaA, E.; Salminen, H.; SAarenmaA, H.; VÄKeVÄ, J. (1996): LIGNUM: A tree model based on simple structural units. Annals of Botany, 77, 87-98. 
PertTUnen, J.; SievÄNEN, R.; NiKInMAA, E. (1998): LIGNUM: a model combining the structure and the functioning of trees. Ecological Modelling, 108, 189-198.

PFREUNDT, J. (1988): Modellierung der räumlichen Verteilung von Strahlung, Photosynthesekapazität und Produktion in einem Fichtenbestand und ihrer Beziehung zur Bestandesstruktur. Berichte des Forschungszentrums Waldökosysteme, Reihe A, Band 39, Göttingen.

Pfreundt, J.; SLOBODA, B. (1996): The Relation of Local Stand Structure to Photosynthetic Capacity in a Spruce Stand: A Model Calculation. Lesnictvi / Forestry, 42, 149-160.

POMMERENING, A. (2000): Neue Methoden zur räumlichen Reproduktion von Waldbeständen und ihre Bedeutung für forstliche Inventuren und deren Fortschreibung, Allgemeine Forst und Jagdzeitung, 171 (9-10), 164-170.

Press, W.H.; Teukolsky, S.A.; VetTerling, W.T.; FlanNery, B.P. (1992): Numerical Recipes in $\mathrm{C}-$ The Art of Scientific Computing. Cambridge University Press, $994 \mathrm{p}$.

PRETZSCH, H. (1990a): Analyse der Bestandesstruktur als Grundlage für die Entwicklung eines Wachstumssimulators für Mischbestände. Tagungsberichte der Arbeitsgruppe Biometrie in der Ökologie, 1, 52-71.

PRETZSCH, H. (1990b): Strukturparameter zur Steuerung von Wuchsmodellen für Rein- und Mischbestände. In: Jahrestagung der Sektion Ertragskunde des Deutschen Verbandes Forstl. Forschungsanstalten, Verden, 80-100.

PretZSCH, H. (1992a): Modellierung der Kronenkonkurrenz von Fichte und Buche in Rein- und Mischbeständen. Allgemeine Forst- und Jagdzeitung, 163, 203-213.

PRETZSCH, H. (1992b): Kronenformen und ihre Veränderung unter Konkurrenz. In: Jahrestagung der Sektion Ertragskunde des Deutschen Verbandes Forstl. Forschungsanstalten, Grillenburg, 40 61.

PRUSINKIEWICZ, P. (1987): Applications of L-systems to computer imagery. Lecture Notes in Computer Science, 291, 534-548.

PRUSINKIEWICZ, P. (1994): Visual Models of Morphogenesis. Artificial Life, 1, Nr. 1/2, 61-74, MIT.

PRUSINKIEWICZ, P. (1997): A look at the visual modeling of plants using L-systems. In: Hofestädt R., Lengauer T., Löffler M., Schomburg D. (Eds.), Bioinformatics - Proceedings GCB'96, Springer, Berlin, Lecture Notes in Computer Science, 1278, 11-29.

Prusinkiewicz, P.; LindenMAYER, A. (1990): The algorithmic beauty of plants. New York: Springer. $\mathrm{X}+228 \mathrm{p}$.

PRUSinKiewicz, P.; LindenMaYeR, A.; HANAN, J. (1988): Developmental models of herbaceous plants for computer imagery purposes. Computer Graphics (ACM / SIGGRAPH), 22 (4), 141150 .

PRUSinkiewicz, P.; JAMES, M., AND MĚCH, R. (1994a): Synthetic topiary. Computer Graphics Proceedings, Annual Conference Ser., 1994, 351-358.

Prusinkiewicz, P.; RemPhreY, W.R.; Davidson, C.G.; HAMmel, M.S. (1994b): Modeling the architecture of expanding Fraxinus pennsylvanica shoots using L-systems. Can. J. Bot., 72, 701-714. 
Prusinkiewicz, P.; Hammel, M.; Hanan, J.; MĚCH, R. (1997): Visual models of plant development. G. Rosenberg, A. Salomaa (Eds.), Handbook of formal languages. Vol. 3: Beyond Words, 535-597. Springer, Berlin.

Prusinkiewicz, P.; Karwowski, R.; Perttunen, J.; SieVÄnen, R. (1999): Specification of L - a plant-modeling language based on Lindenmayer systems. Version 0.5. Manuscript.

Prusinkiewicz, P.; MÜndermann, L.; KARWOWski, R.; LANE, B. (2001): The use of positional information in the modeling of plants. ACM SIGGRAPH 2001, 289-300.

RAPIDEL, BRUNO (1995): Etude expérimental et simulation des transferts hydriques dans les plantes individuelles. Application au caféier (Coffea arabica L.), Thèse de doctorat, Université de Montpellier.

RAPIDEL, B.; DAUZAT, J.; BERGER, A. (1999): Application of a mock-up based transpiration and photosynthesis model on a Coffee plantation in Costa Rica. In: Multi-strata agroforestry systems with perennial crops, International Symposium, F. Jimémez and J. Beer (Eds), Turrialba, Costa Rica 22-27/02/1999. CATIE; 198-203.

RENSHAW, E. (1985): Computer simulation of Sitka spruce: Spatial branching models for canopy growth and root structure. IMA Journal of Mathematics applied in Medicine and Biology, 2, $183-200$.

RICHARDS, P.W. (1996): The tropical rain forest: an ecological study. Cambridge University Press, 74 p.

RICHTER, J.P. (1970): The notebooks of Leonardo da Vinci (1452-1519), compiled and edited from the original manuscripts. Dover, New York (Reprint of a work originally published by Sampson Low, Marston Searle and Rivington, London 1883).

ROBERTS, J. (1976): An examination of the quantity of water stored in mature Pinus sylvestris L. trees. Journal of Experimental Botany, 42, 123-130.

Roloff, A. (1984): Morphologie der Verzweigung von Fagus sylvatica L. (Rotbuche) als Grundlage zur Beurteilung von Triebanomalien und Kronenschäden. Berichte des Forschungszentrumg Walökosysteme / Waldsterben, 18, Göttingen.

RUNNING, S.W.; WARING, R.H.; RYDELL, R.A. (1975): Physiological control of water flux in conifers. A computer simulation model. Oecologia 18, 1-16.

SAARENMAA, H. (1992): Object-oriented design of topological tree and forest models. In: A. Teller, P. Mathy, J.N.R. Jeffers (eds.) Responses of forest ecosystems to environmental changes. London. 957-958.

SAarenmaA, H.; PertTunen, J.; VÄKeVÄ, J.; NikUlA, A. (1994a): Object-oriented Modeling of Tasks and Agents in Integrated Forest Health Management. AI Applications, Vol. 8, No. 1, $43-59$.

Salminen, H.; SaArenmaA, H.; Perttunen, J.; SievÄnen, R.; NikinmaA, E.; VÄKeVÄ, J. (1994b): Modelling trees with an object-oriented scheme. In: Saarenmaa, H. \& Gimbett, R. (eds.). Object-oriented modelling of natural and artificial agents in ecosystem and natural resource management. Mathematical and Computer Modelling, 20(8), 49-64. 
SAUGIER, B. (1974): Transport de $\mathrm{CO}_{2}$ et de vapeur d'eau à l'interface végétation - atmosphère. Interaction du microclimat avec le comportement physiologique de plantes prairiales. Thèse de doctorat, Université des Sciences et Techniques du Languedoc, Montpellier.

SCHMidT, J.P.; BlEndingeR, C.; LANGE, H. (1995): silVlow - eine Modelldokumentation. In: Dokumentation von Wasserhaushaltsmodellen. Hörmann, G., Schmidt, J. (eds.). Berichte des Forschungszentrums Waldökosysteme, Reihe B, Bd. 42, 71-83, Göttingen.

Schulze, E. D.; Čermak, J.; Matyssek, R.; Penka, M.; Zimmermann, R.; VasiČeK, F.; Gries, W.; KUČERA, J. (1985): Canopy transpiration and water fluxes in the xylem of the trunk of Larix and Picea trees - a comparison of xylem flow, porometer and cuvette measurements. Oecologia, 66, 475-483.

SHINOZAKI, KICHIRO; YODA, KYORI; HOZUMI, KAZUO; KIRA, TATUO (1964): A quantitative analysis of plant form - the pipe model theory. I. Basic analyses. Japanese Journal of Ecology, Vol. 14, Nr. 3, 97-139.

Sievänen, R.; NikinmaA, E.; Hakula, H.; Perttunen, J.; Salminen, H.; SaArenmaA, H.; VÄKEVÄ, J. (1995): Lignum - modeling trees with simple structural units. CSC News 7(2), $20-24$

SinOQUet, HeRVÉ; Le RouX, XAVIER (2000): Short term interactions between tree foliage and the aerial environment: An overview of modelling approaches available for tree structure-function models. Annals of Forest Science, Nr. 57, 477-496.

SPERRY, J.S.; DONNELLY, J.R.; TYREE, M.T. (1988): A method for measuring hydraulic conductivity and embolism in xylem, Plant Cell Environment, 11, 35-40.

SPITTERS, C.J.T.; TOussainT, H.A.J.M.; GoudRIAAN, J. (1986): Separating the diffuse and direct component of global radiation and its implication for modelling canopy photosynthesis. Part I: components of incoming radiation. Agricultural and Forest Meteorology, 38, 217-229.

SUZUKI, T.; OOSAKI, K.; SATO, H.; YAMAMOTO, Y. (1992): コンピュータ グラフィックス による トドマツ単木の形状表示 (A representation method for todo-fir shapes using computer graphics). Journal of the Japanese Forestry Society, 74(6), 504-508.

TAKENAKA, AKIO (1994): A simulation model of tree architecture development based on growth response to local light environment. Journal of Plant Research, 197, 321-330.

Tausend, P.; Meinzer, F.C.; Goldstein, G. (2000): Control of transpiration in three coffee cultivars: the role of hydraulic and crown architecture. Trees, 14, 181-190.

THOM, A.S. (1975): Momentum, mass and heat exchange of plant communities. In: J.L. Monteith (Ed.), Vegetation and the atmosphere. Vol. I: Principles, Academic Press, London, 57-109.

ThORNLEY, J.H.M. (1976): Photosynthesis. In: J.H.M. Thornley (ed.), Mathematical models in plant physiology, Academic Press, London, 92-110.

TOMLINSON, P.B. (1983): Tree architecture. American Scientist, 71, 141-149.

TSEL'NIKER, Y. (1994): Structure of spruce crown. Lesovedenie, No. 4, 35-44.

TSEL'NIKER, Y. (1995): Der Einfluß der Lichtintensität auf die Kronenstrukturparameter der Fichte. Lesovedenie 1995, 73-78. 
TYREE, M.T. (1988): A dynamic model for water flow in a single tree: evidence that models must account for hydraulic architecture. Tree Physiology, 4, 195-217.

TYREE, M.T. (1993): Theory of vessel-length determination: the problem of nonrandom vessel ends. Canadian Journal of Botany, 71, 297-302.

TYREE, M.T.; SPERRY, J.S. (1988): Do woody plants operate near the point of catastrophic xylem dysfunction caused by dynamic water stress? Answers from a model. Plant Physiology 88, 574-580.

UlRICH, B. (1981): Eine ökosystemare Hypothese zum Tannensterben. Forstwiss. Centralblatt., 100, $288-236$.

VAN DEN HONERT, T.H. (1948): Water transports in plants as a catenary process. Discuss. Faraday Society, 3, 146-153.

VAN HeESCH, D. (2001): User Manual for Doxygen 1.2.10. Available on-line unter http://www.doxygen.org.

WoOdCock, H.; Vollenweider, P.; Bubs, R.; HOFER, R.-M. (1995): Crown alterations induced by decline: a study of relationships between growth rate and crown morphology in beech (Fagus sylvatica L.). Trees, 9, 279-288.

YANG, S.; TYREE, M.T. (1993): Hydraulic architecture of Acer saccharum and A. rubrum: comparison of branches to whole tree and the contribution of leaves to hydraulic resistance. Journal of Experimental Botany, Vol. 45, No. 271, 179-186.

Yang, S.; LiU, X.; Tyree, M.T. (1998): A Model of Stomatal Conductance in Sugar Maple (Acer saccharum Marsh). Journal of Theoretical Biology, 191, 197-211.

Zhang, H.; NoBel, P.S. (1996): Dependency of Ci/Ca and leaf transpiration on the vapour pressure deficit. Australian Journal of Plant Physiology, 23, 561-568. 


\section{APPENDIX 1}

Original $d t d$ structure files for the sample trees and their graphical representation with GROGRA.

ejc:

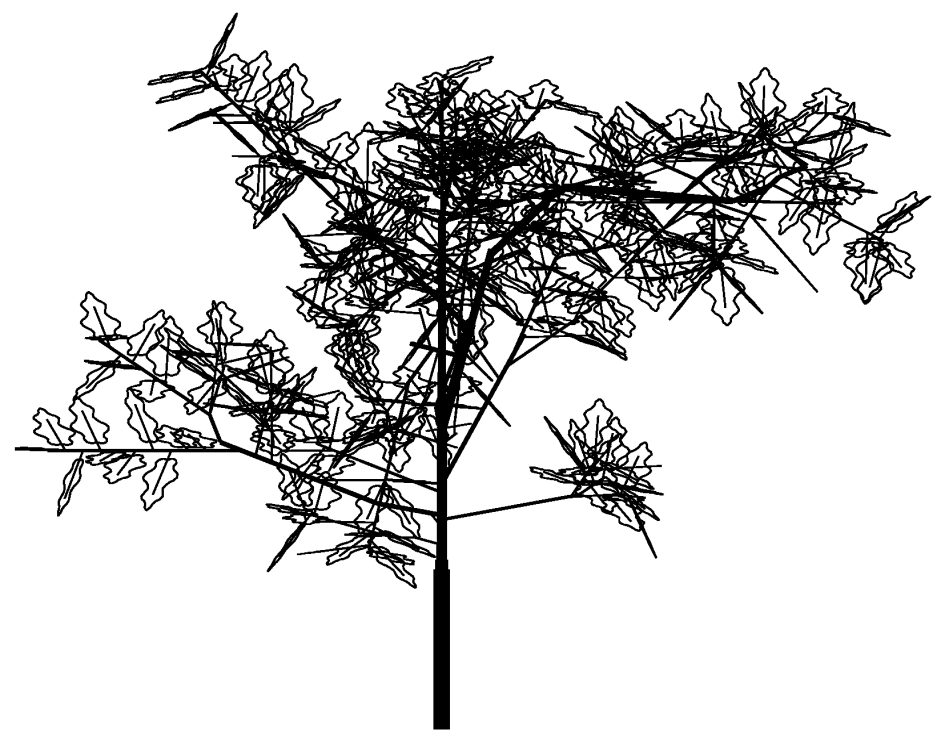

\leafobject eblatt.Isy veg 2,

$\backslash$ leafarea 1337,

$\backslash$ leaflength 65.9,

$\backslash$ phyllotaxy spiral,

Imin_intn 10,

E0 L150 \#\#

$1.1 \quad$ L48 \#E0

1. 2

L4 2

E0 A150 R1

$\# 1.1$ V $\quad$ RI

$\# 1.2 \mathrm{~V}$

$\# 1.3 \mathrm{~V}$

L28

$\# 1.2$ A32

L89 \#1.5 V

L34 \#1.6 V $\quad$ R3

$\begin{array}{lll}\# 1.6 & \text { V } & \text { R3 } \\ \# 1.6 & \text { A76 } & -\end{array}$

$\begin{array}{llll}\text { L56 } & \# 1.6 & \text { A76 } \\ \text { L55 } & \# 1.6 & \text { A82 } & +\end{array}$

1.8

$\begin{array}{llll}\text { L55 } & \# 1.6 & \text { A82 } & + \\ \text { L163 } & \# 1.3 & \text { A37 } & \text { R8 }\end{array}$

1.10

L163 \#1.3 A37 R8

W60

D14.2

W15

D9. 4

D8. 6

D8. 0

D2. 6

W75 D2.6

D2. 6

W30 D2.0 B5

W10 D1.5 B4

W50 D1.5 B8

W40 D3.5 <Broken tip> 


\begin{tabular}{|c|c|c|c|c|c|c|c|c|}
\hline 1.11 & L95 & $\# 1.10$ & A128 & R6 & W30 & $\mathrm{D} 2.2$ & B5 & \\
\hline 1.12 & L114 & $\# 1.10$ & A138 & RI & W4 0 & D2. 6 & B9 & \\
\hline 1.13 & L80 & $\# 1.10$ & A114 & + & W60 & $\mathrm{D} 2.0$ & & \\
\hline 1.14 & L188 & $\# 1.3$ & A 52 & R5 & W15 & D7. 2 & & \\
\hline 1.15 & L292 & $\# 1.14$ & $\mathrm{~V}$ & & & D6. 8 & & \\
\hline 1.16 & L120 & $\# 1.15$ & V & $\mathrm{R} 8$ & W10 & $\mathrm{D} 4.2$ & B10 & \\
\hline 1.17 & L22 & $\# 1.14$ & A177 & - & W60 & $\mathrm{D} 1.4$ & B3 & \\
\hline 1.18 & L127 & $\# 1.15$ & A22 7 & - & W60 & $\mathrm{D} 2.3$ & B8 & \\
\hline 1.19 & L183 & $\# 1.15$ & A 262 & R5 & W60 & D3. 3 & B10 & \\
\hline 1.20 & L153 & $\# 1.15$ & A2 73 & $\mathrm{R} 8$ & W60 & D2. 8 & B6 & \\
\hline 1.21 & L180 & $\# 1.15$ & A2 83 & - & W60 & D3. 2 & B9 & \\
\hline 1.22 & L58 & $\# 1.15$ & A2 88 & $\mathrm{R} 1$ & W60 & $\mathrm{D} 2.2$ & B5 & \\
\hline 1.23 & L192 & $\# 1.15$ & A2 82 & + & W50 & D3. 4 & B10 & \\
\hline 1.24 & L152 & $\# 1.15$ & A2 44 & $\mathrm{R} 1$ & W60 & D2. 6 & B8 & \\
\hline $1.24 \mathrm{a}$ & L116 & $\# 1.15$ & A198 & + & W45 & D2. 4 & B8 & \\
\hline 1.25 & L122 & $\# 1.15$ & A183 & R1 & W4 0 & D2. 5 & & \\
\hline 1.26 & L38 & $\# 1.25$ & A122 & - & W50 & $\mathrm{D} 1.4$ & B4 & \\
\hline 1.27 & L38 & $\# 1.25$ & A 122 & R5 & W60 & $\mathrm{D} 1.4$ & B2 & \\
\hline 1.28 & L52 & $\# 1.25$ & A 112 & + & W30 & $\mathrm{D} 1.6$ & B6 & \\
\hline 1.29 & L84 & $\# 1.3$ & A2 6 & + & W60 & $\mathrm{D} 2.3$ & & \\
\hline 1.30 & L46 & $\# 1.29$ & $\mathrm{~V}$ & & & D1.9 & B6 & \\
\hline 1.31 & L60 & $\# 1.2$ & A3 8 & + & W75 & D3.9 & & \\
\hline 1.32 & L162 & $\# 1.31$ & $\mathrm{~V}$ & R7 & W15 & D3. 9 & & $<$ Broken tip $>$ \\
\hline 1.33 & L93 & $\# 1.32$ & A126 & - & W70 & $\mathrm{D} 1.9$ & B7 & \\
\hline 1.34 & L38 & $\# 1.32$ & A162 & - & W45 & $\mathrm{D} 2.9$ & & \\
\hline 1.35 & L115 & $\# 1.34$ & A3 8 & R6 & W30 & $\mathrm{D} 1.9$ & B5 & \\
\hline 1.36 & L74 & $\# 1.34$ & A38 & $\mathrm{R} 8$ & W30 & $\mathrm{D} 1.5$ & B5 & \\
\hline 1.37 & L140 & $\# 1.34$ & A3 8 & + & W30 & $\mathrm{D} 2.5$ & B7 & \\
\hline 1.38 & L174 & $\# 1.32$ & $\mathrm{~A} 140$ & + & W30 & D2. 9 & B10 & \\
\hline 1.39 & L80 & $\# 1.32$ & A109 & R1 & W90 & $\mathrm{D} 1.6$ & B6 & \\
\hline 1.40 & L17 & $\# 1.2$ & A22 & $\mathrm{R} 2$ & W60 & $\mathrm{D} 1.1$ & & \\
\hline 1.41 & L30 & $\# 1.1$ & A4 6 & + & w60 & $\mathrm{D} 1.8$ & & $<$ Broken tip > \\
\hline 1.42 & L20 & $\# 1.41$ & A2 8 & + & W30 & $\mathrm{D} 1.8$ & & \\
\hline 1.43 & L56 & $\# 1.42$ & V & - & W30 & $\mathrm{D} 1.8$ & & \\
\hline 1.44 & L36 & $\# 1.43$ & V & & & $\mathrm{D} 1.7$ & B5 & \\
\hline 2.1 & L9 & \#E0 & V & & & $\mathrm{D} 11.4$ & & \\
\hline 2.2 & L123 & $\# 2.1$ & V & & & D8. 9 & & \\
\hline 2.3 & L62 & $\# 2.2$ & V & R3 & W15 & D8. 5 & & \\
\hline 2.4 & L40 & $\# 2.3$ & V & & & D7. 5 & & \\
\hline 2.5 & L72 & $\# 2.4$ & V & & & D6. 6 & & $<$ Broken tip $>$ \\
\hline 2.6 & L22 & $\# 2.2$ & A108 & R4 & W60 & $\mathrm{D} 1.8$ & & $<$ Broken tip $>$ \\
\hline 2.7 & L12 & $\# 2.6$ & A19 & R1 & W60 & $\mathrm{D} 1.7$ & & \\
\hline 2.8 & L22 & \#2.7 & $\mathrm{V}$ & + & W10 & $\mathrm{D} 1.7$ & & \\
\hline 2.9 & L39 & $\# 2.8$ & $\mathrm{~V}$ & - & W10 & $\mathrm{D} 1.7$ & & \\
\hline 2.10 & L90 & $\# 2.5$ & A67 & R5 & W30 & D6. 3 & & \\
\hline 2.11 & L125 & $\# 2.10$ & $\mathrm{~V}$ & R5 & W50 & D6. 3 & & \\
\hline 2.12 & L26 & $\# 2.11$ & $\mathrm{~V}$ & R7 & W70 & D6.0 & & \\
\hline 2.13 & L60 & $\# 2.12$ & $\mathrm{~V}$ & & & D5 . 8 & & \\
\hline 2.14 & L95 & $\# 2.13$ & $\mathrm{~V}$ & R1 & W10 & D4. 4 & & \\
\hline 2.15 & L27 & \#2.14 & $\mathrm{V}$ & R1 & W10 & D 4.3 & & \\
\hline 2.16 & L75 & \#2.15 & V & R5 & W10 & D4.0 & & \\
\hline 2.17 & L123 & \#2.16 & $\mathrm{V}$ & R7 & W30 & D3. 9 & & \\
\hline 2.18 & L114 & \#2.17 & $\mathrm{V}$ & & & D2. 8 & B9 & \\
\hline 2.19 & L13 & $\# 2.11$ & A120 & R4 & W120 & D3.0 & & \\
\hline 2.20 & L29 & \#2.19 & $\mathrm{V}$ & & & D3.0 & & \\
\hline 2.21 & L23 & $\# 2.20$ & A29 & + & W5 & D2. 2 & & \\
\hline 2.22 & L16 & $\# 2.21$ & $\mathrm{~V}$ & & & $\mathrm{D} 2.2$ & & \\
\hline 2.23 & L27 & $\# 2.22$ & V & & & D2. 0 & & \\
\hline 2.24 & L45 & $\# 2.23$ & V & & & D1.9 & & $<$ Broken tip $>$ \\
\hline 2.25 & L15 & $\# 2.24$ & A24 & + & W4 5 & $\mathrm{D} 1.0$ & B4 & \\
\hline 2.26 & L10 & $\# 2.24$ & A16 & - & W4 5 & D0. 8 & B2 & \\
\hline 2.27 & L49 & $\# 2.20$ & A29 & - & W4 0 & D2. 0 & & \\
\hline 2.28 & L29 & \#2.27 & V & & & D2. 0 & & \\
\hline 2.29 & L22 & $\# 2.28$ & A22 & + & W70 & $\mathrm{D} 1.4$ & & \\
\hline 2.30 & L4 & $\# 2.29$ & V & & & $\mathrm{D} 1.4$ & B3 & \\
\hline 2.31 & L22 & $\# 2.28$ & A12 & R5 & W60 & $\mathrm{D} 1.3$ & & \\
\hline 2.32 & L26 & $\# 2.11$ & A125 & R6 & W2 0 & D2. 2 & & \\
\hline
\end{tabular}




\begin{tabular}{|c|c|c|c|c|c|c|c|}
\hline 2.33 & L33 & $\# 2.32 \mathrm{~V}$ & & & $\mathrm{D} 2.2$ & & \\
\hline 2.34 & L4 8 & \#2.33 A23 & R5 & W30 & D1.9 & & \\
\hline 2.35 & L18 & $\# 2.34 \mathrm{~V}$ & & & D1.8 & & \\
\hline 2.36 & L10 & $\# 2.35 \mathrm{~V}$ & & & D1.3 & & \\
\hline 2.37 & L20 & $\# 2.36 \mathrm{~V}$ & & & D1.2 & & \\
\hline 2.38 & L57 & \#2.33 A31 & - & W70 & D1.8 & & \\
\hline 2.39 & L10 & $\# 2.38 \mathrm{~V}$ & & & D1.0 & & \\
\hline 2.40 & L17 & \#2.13 A4 7 & - & w42 & $\mathrm{D} 2.8$ & & \\
\hline 2.41 & L25 & $\# 2.40 \mathrm{~V}$ & & & $\mathrm{D} 2.5$ & & $<$ Broken tip $>$ \\
\hline 2.42 & L35 & \#2.41 A17 & R4 & W30 & D2. 3 & & \\
\hline 2.43 & L88 & $\# 2.42 \mathrm{~V}$ & & & $\mathrm{D} 2.2$ & & \\
\hline 2.44 & L31 & $\# 2.43 \mathrm{~V}$ & & & $\mathrm{D} 2.0$ & B4 & \\
\hline 2.45 & L11 & $\# 2.43$ A68 & R6 & W8 0 & $\mathrm{D} 1.0$ & B2 & \\
\hline 2.46 & L10 & $\# 2.43$ А 30 & R7 & W8 0 & $\mathrm{D} 1.0$ & $\mathrm{~B} 1$ & \\
\hline 2.47 & L10 & $\# 2.41$ A18 & R8 & W60 & D1.0 & & $<$ Broken tip $>$ \\
\hline 2.48 & L18 & \#2.41 A12 & R7 & W90 & D1.8 & & \\
\hline 2.49 & L45 & $\# 2.48 \mathrm{~V}$ & & & $\mathrm{D} 1.8$ & & \\
\hline 2.50 & L23 & $\# 2.49 \mathrm{~V}$ & & & $\mathrm{D} 1.7$ & B6 & \\
\hline 2.51 & L115 & $\# 2.13$ A55 & R3 & W4 0 & $\mathrm{D} 2.3$ & & $<$ Broken tip $>$ \\
\hline 2.52 & L15 & $\# 2.51$ A110 & R8 & W4 0 & D1.0 & & $<$ Broken tip $>$ \\
\hline 2.53 & L20 & $\# 2.15$ A7 & R4 & W60 & $\mathrm{D} 2.0$ & & \\
\hline 2.54 & L70 & $\# 2.53 \mathrm{~V}$ & & & $\mathrm{D} 1.9$ & & \\
\hline 2.55 & L34 & $\# 2.54 \mathrm{~V}$ & & & $\mathrm{D} 1.7$ & B6 & \\
\hline 2.56 & L11 & $\# 2.16$ A43 & + & W60 & $\mathrm{D} 1.2$ & & \\
\hline 2.57 & L25 & $\# 2.56 \mathrm{~V}$ & & & $\mathrm{D} 1.0$ & B2 & \\
\hline 2.58 & L12 & \#2.17 A57 & R1 & W90 & $\mathrm{D} 1.1$ & B2 & \\
\hline 2.59 & L49 & \#2.17 A73 & R4 & W8 0 & $\mathrm{D} 1.5$ & B6 & \\
\hline 2.60 & L53 & \#2.17 A98 & R2 & W90 & $\mathrm{D} 1.7$ & B2 & \\
\hline 2.61 & L68 & $\# 2.16$ A55 & - & W60 & $\mathrm{D} 2.0$ & & \\
\hline 2.62 & L43 & $\# 2.61 \mathrm{~V}$ & & & $\mathrm{D} 1.6$ & B5 & \\
\hline 2.63 & L21 & \#2.15 A17 & R6 & W8 0 & $\mathrm{D} 2.2$ & & \\
\hline 2.64 & L59 & $\# 2.63 \mathrm{~V}$ & R2 & W10 & $\mathrm{D} 2.0$ & & \\
\hline 2.65 & L51 & $\# 2.64 \mathrm{~V}$ & & & $\mathrm{D} 2.0$ & B7 & \\
\hline 2.66 & L4 & $\# 2.64$ A40 & - & w42 & $\mathrm{D} 1.0$ & $\mathrm{~B} 1$ & \\
\hline 2.67 & L70 & $\# 2.13$ A56 & R7 & W55 & $\mathrm{D} 2.8$ & & $<$ Broken tip $>$ \\
\hline 2.68 & L8 & \#2.67 A18 & R2 & W60 & D1. 5 & & \\
\hline 2.69 & L17 & $\# 2.68 \mathrm{~V}$ & & & $\mathrm{D} 1.5$ & & \\
\hline 2.70 & L29 & $\# 2.69 \mathrm{~V}$ & & & $\mathrm{D} 1.5$ & & \\
\hline 2.71 & L12 & $\# 2.70 \mathrm{~V}$ & & & D1. 4 & B3 & \\
\hline 2.72 & L38 & \#2.67 A67 & R7 & W30 & $\mathrm{D} 2.2$ & & \\
\hline 2.73 & L30 & $\# 2.72 \mathrm{~V}$ & & & $\mathrm{D} 1.9$ & & \\
\hline 2.74 & L38 & $\# 2.73 \mathrm{~V}$ & & & $\mathrm{D} 1.7$ & & \\
\hline 2.75 & L24 & $\# 2.74 \mathrm{~V}$ & & & D1.3 & B4 & \\
\hline 2.76 & L27 & \#2.73 A23 & R2 & W8 0 & $\mathrm{D} 1.4$ & & \\
\hline 2.77 & L24 & $\# 2.76 \mathrm{~V}$ & & & $\mathrm{D} 1.4$ & B4 & \\
\hline 2.78 & L10 & $\# 2.67$ A38 & R6 & W50 & $\mathrm{D} 1.3$ & & \\
\hline 2.79 & L15 & $\# 2.78 \mathrm{~V}$ & & & $\mathrm{D} 1.3$ & & \\
\hline 2.80 & L15 & $\# 2.79 \mathrm{~V}$ & & & $\mathrm{D} 1.3$ & & \\
\hline 2.81 & L19 & $\# 2.80 \mathrm{~V}$ & & & $\mathrm{D} 1.3$ & B4 & \\
\hline 2.82 & L2 8 & $\# 2.13$ A2 6 & R8 & W110 & $\mathrm{D} 2.6$ & & \\
\hline 2.83 & L18 & $\# 2.82 \mathrm{~V}$ & R5 & W30 & $\mathrm{D} 2.6$ & & \\
\hline 2.84 & L34 & $\# 2.83 \mathrm{~V}$ & & & $\mathrm{D} 2.6$ & & \\
\hline 2.85 & L12 & $\# 2.84$ A10 & R8 & W90 & $\mathrm{D} 1.3$ & & \\
\hline 2.86 & L13 & $\# 2.85 \mathrm{~V}$ & & & $\mathrm{D} 1.3$ & B5 & \\
\hline 2.87 & L4 6 & \#2.84 A15 & R3 & W60 & D1.5 & & $<$ Broken tip $>$ \\
\hline 2.88 & L15 & $\# 2.87$ A35 & R1 & W60 & $\mathrm{D} 1.3$ & B4 & \\
\hline 2.89 & L8 & \#2.87 A21 & R5 & W30 & D0.8 & B2 & \\
\hline 2.90 & L18 & \#2.84 A20 & R6 & W60 & D1.9 & & \\
\hline 2.91 & L9 & $\# 2.90 \mathrm{~V}$ & & & D1.0 & B1 & \\
\hline 2.92 & L4 & $\# 2.90$ a14 & R1 & W45 & D1.0 & B2 & \\
\hline 2.93 & L8 & \#2.10 A88 & R2 & W90 & D1.6 & & \\
\hline 2.94 & L13 & $\# 2.93 \mathrm{~V}$ & & & $\mathrm{D} 1.6$ & & $<$ Broken tip $>$ \\
\hline 2.95 & L86 & $\# 2.10$ A88 & R1 & W90 & D2.0 & & $\langle$ Broken tip $\rangle$ \\
\hline 2.96 & L7 & \#2.95 A81 & + & W30 & D1.8 & & \\
\hline 2.97 & L18 & $\# 2.96 \mathrm{~V}$ & & & D1.8 & & $<$ Broken tip $>$ \\
\hline 2.98 & L12 & $\# 2.10$ A88 & R8 & W120 & $\mathrm{D} 2.0$ & & \\
\hline 2.99 & L22 & $\# 2.98 \mathrm{~V}$ & & & D1.8 & & \\
\hline
\end{tabular}




\begin{tabular}{|c|c|c|c|c|c|c|c|c|}
\hline 2.100 & L34 & $\# 2.99 \mathrm{~V}$ & & & $\mathrm{D} 1.8$ & & & \\
\hline 2.101 & L21 & $\# 2.100$ & $\mathrm{~V}$ & $\mathrm{R} 1$ & W4 0 & $\mathrm{D} 1.7$ & B5 & \\
\hline 2.102 & L70 & $\# 2.4 \quad$ A35 & R1 & W60 & $\mathrm{D} 4.6$ & & & \\
\hline 2.103 & L54 & \#2.102 & V & & & D3. 6 & & \\
\hline 2.104 & L124 & $\# 2.103$ & V & & & D3.0 & & \\
\hline 2.105 & L95 & $\# 2.104$ & V & & & D2. 8 & B10 & \\
\hline 2.106 & L19 & \#2.102 & A66 & R5 & W80 & $\mathrm{D} 1.6$ & & \\
\hline 2.107 & L39 & $\# 2.106$ & V & & & D1. 6 & & \\
\hline 2.108 & L22 & \#2.107 & V & & & $\mathrm{D} 1.6$ & B6 & \\
\hline 2.109 & L28 & $\# 2.102$ & A 67 & R7 & W60 & $\mathrm{D} 2.3$ & & \\
\hline 2.110 & L65 & \#2.109 & V & & & $\mathrm{D} 2.1$ & & \\
\hline 2.111 & L35 & $\# 2.110$ & V & & & $\mathrm{D} 1.8$ & B6 & \\
\hline 2.112 & L25 & $\# 2.110$ & A58 & - & w32 & $\mathrm{D} 1.4$ & B4 & \\
\hline 2.113 & L23 & $\# 2.103$ & A29 & R5 & W60 & $\mathrm{D} 1.8$ & & \\
\hline 2.114 & L43 & $\# 2.113$ & $\mathrm{~V}$ & & & $\mathrm{D} 1.6$ & B5 & \\
\hline 2.115 & L14 & $\# 2.104$ & A90 & $\mathrm{R} 4$ & W70 & $\mathrm{D} 1.1$ & B2 & \\
\hline 2.116 & L22 & $\# 2.104$ & A 114 & R5 & W70 & $\mathrm{D} 1.4$ & B4 & \\
\hline 2.117 & L23 & $\# 2.104$ & A121 & $\mathrm{R} 2$ & W90 & $\mathrm{D} 1.4$ & B4 & \\
\hline 2.118 & L15 & $\# 2.102$ & A65 & R1 & W80 & $\mathrm{D} 1.6$ & & \\
\hline 2.119 & L28 & \#2.118 & $\mathrm{V}$ & & & $\mathrm{D} 1.6$ & & \\
\hline 2.120 & L38 & $\# 2.119$ & $\mathrm{~V}$ & & & D1. 5 & B5 & \\
\hline 2.121 & L48 & $\# 2.4 \quad$ A8 & R1 & W90 & D3. 9 & & $<$ Broken tip $>$ & \\
\hline 2.122 & L17 & $\# 2.121$ & A 27 & R5 & W70 & $\mathrm{D} 2.8$ & & \\
\hline 2.123 & L25 & $\# 2.122$ & V & & & $\mathrm{D} 2.8$ & <Broken & tips \\
\hline 2.124 & L46 & $\# 2.123$ & A 21 & $\mathrm{R} 6$ & W30 & $\mathrm{D} 2.6$ & & \\
\hline 2.125 & L80 & \#2.124 & $\mathrm{V}$ & & & $\mathrm{D} 2.3$ & & \\
\hline 2.126 & L46 & $\# 2.125$ & $\mathrm{~V}$ & & & D1. 9 & B7 & \\
\hline 2.127 & L13 & $\# 2.125$ & A 45 & + & W60 & $\mathrm{D} 1.1$ & B4 & \\
\hline 2.128 & L38 & $\# 2.123$ & A23 & R1 & W60 & D1. 5 & <Broken & tips \\
\hline 2.129 & L22 & $\# 2.123$ & A17 & R3 & W4 0 & D1. 7 & & \\
\hline 2.130 & L2 8 & $\# 2.129$ & V & & & $\mathrm{D} 1.6$ & & \\
\hline 2.131 & L8 & $\# 2.130$ & A2 8 & + & W60 & D1. 5 & B2 & \\
\hline 2.132 & L17 & $\# 2.130$ & A2 8 & $\mathrm{R} 6$ & W60 & $\mathrm{D} 1.6$ & B4 & \\
\hline 2.133 & L12 & $\# 2.130$ & A2 8 & R1 & W60 & D1. 5 & B3 & \\
\hline 2.134 & L17 & $\# 2.121$ & A29 & R1 & W60 & D1. 8 & & \\
\hline 2.135 & L22 & $\# 2.134$ & V & & & D1. 7 & <Broken & tips \\
\hline $2.136 a$ & L7 & $\# 2.135$ & A17 & R5 & W70 & D1. 5 & & \\
\hline 2.136 & L17 & $\# 2.136 a$ & V & & & $\mathrm{D} 1.3$ & & \\
\hline 2.137 & L22 & \#2.136 & $\mathrm{V}$ & & & D1. 1 & B5 & \\
\hline 2.138 & L15 & $\# 2.121$ & $\mathrm{~A} 22$ & R3 & W90 & $\mathrm{D} 2.7$ & & \\
\hline 2.139 & L22 & $\# 2.138$ & V & & & $\mathrm{D} 2.5$ & & \\
\hline 2.140 & L39 & \#2.139 & $\mathrm{V}$ & & & $\mathrm{D} 2.5$ & <Broken & tips \\
\hline 2.141 & L52 & $\# 2.140$ & A4 & R5 & W4 5 & D1. 6 & & \\
\hline 2.142 & L32 & $\# 2.141$ & $\mathrm{~V}$ & & & $\mathrm{D} 1.6$ & B7 & \\
\hline 2.143 & L66 & $\# 2.140$ & $\mathrm{~A} 2$ & R1 & W45 & $\mathrm{D} 1.8$ & & \\
\hline 2.144 & L66 & $\# 2.143$ & $\mathrm{~V}$ & & & $\mathrm{D} 1.8$ & B5 & \\
\hline 2.145 & L29 & $\# 2.2 \quad A 115$ & - & W10 & D3. 5 & & & \\
\hline 2.146 & L35 & $\# 2.145$ & $\mathrm{~V}$ & $\mathrm{R} 1$ & W30 & D3. 4 & & \\
\hline $2.146 a$ & L9 & $\# 2.146$ & $\mathrm{~V}$ & + & W60 & D3 .4 & & \\
\hline 2.147 & L22 & $\# 2.146 a$ & $\mathrm{~V}$ & & & $\mathrm{D} 2.9$ & & \\
\hline 2.148 & L15 & \#2.147 & $\mathrm{V}$ & & & D3. 1 & & \\
\hline 2.149 & L15 & $\# 2.148$ & $\mathrm{~V}$ & R7 & W4 5 & D1. 7 & & \\
\hline 2.150 & L7 & $\# 2.149$ & $\mathrm{~V}$ & & & D1. 7 & <Broken & tips \\
\hline 2.151 & L9 & $\# 2.146$ & A34 & & wo & $\mathrm{D} 2.1$ & & \\
\hline 2.152 & L25 & \#2.151 & $\mathrm{V}$ & & & $\mathrm{D} 2.1$ & <Broken & tips \\
\hline 2.153 & L10 & $\# 2.152$ & $\mathrm{~A} 22$ & - & W60 & D1. 9 & <Broken & tips \\
\hline 2.154 & L4 5 & \#2.153 & A 8 & + & W60 & D1. 8 & <Broken & tip $>$ \\
\hline 2.155 & L10 & $\# 2.154$ & A4 3 & + & W60 & D1. 7 & <Broken & tips \\
\hline 2.156 & L25 & \#2. 148 & A15 & R5 & W4 5 & D1. 4 & <Broken & tips \\
\hline 2.157 & L26 & $\# 2.148$ & A15 & $\mathrm{R} 1$ & W4 5 & D2. 7 & & \\
\hline 2.158 & L78 & $\# 2.157$ & $\mathrm{~V}$ & R5 & W10 & D2. 5 & & \\
\hline 2.159 & L104 & $\# 2.158$ & $\mathrm{~V}$ & & & D2. 5 & B8 & \\
\hline 2.160 & L35 & \#2.158 & A50 & R5 & W90 & $\mathrm{D} 1.2$ & B5 & \\
\hline 2.161 & L28 & $\# 2.145$ & A8 & + & W90 & D1. 7 & & \\
\hline 2.162 & L33 & $\# 2.161$ & $\mathrm{~V}$ & & & $\mathrm{D} 1.6$ & $<$ Broken & tips \\
\hline 2.163 & L15 & $\# 2.161$ & $\mathrm{~A} 12$ & R5 & W60 & D1. 1 & & \\
\hline 2.164 & L10 & \#2.162 & A2 8 & R5 & W60 & Do. 9 & & \\
\hline
\end{tabular}




$\begin{array}{lllllll}2.165 & \text { L33 } & \# 2.161 & \text { A22 } & \text { R1 } & \text { W60 } & \text { D1.3 } \\ 2.166 & \text { L13 } & \# 2.161 & \text { A5 } & + & \text { W30 } & \text { D0.9 }\end{array}$

ejd:

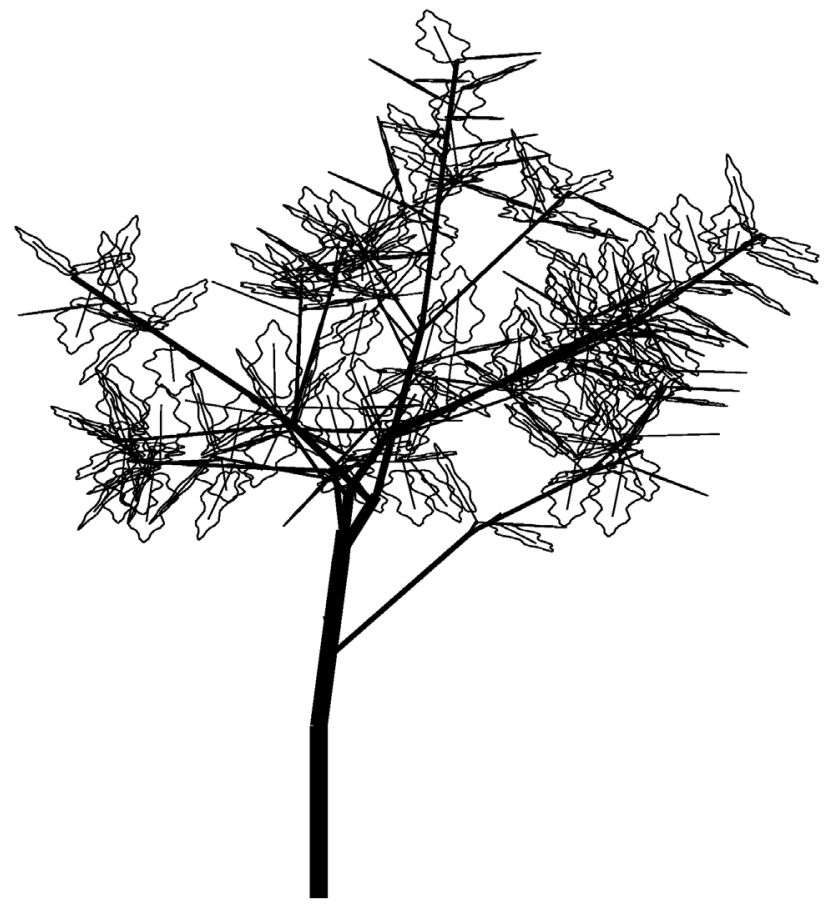

\leafobject eblatt.lsy veg 2,

$\backslash$ leafarea 1337

$\backslash$ leaflength 65.9,

$\backslash$ phyllotaxy spiral,

Imin_intn 10,

\begin{tabular}{|c|c|c|c|c|c|c|c|c|}
\hline EO & L125 & \#\# & & & & D12.0 & & \\
\hline E1 & L40 & \#E0 & $\mathrm{V}$ & R2 & W10 & D11.7 & & \\
\hline $\mathrm{E} 2$ & L85 & \#E1 & $\mathrm{V}$ & & & D11.7 & & \\
\hline E3 & L19 & \#Е2 & V & & & $\mathrm{D} 11.2$ & & $<$ Broken tip $>$ \\
\hline 1.1 & L63 & \#E2 & A10 & R6 & W4 0 & D3 .5 & & \\
\hline 1.2 & L73 & \#1.1 & V & & & D3 .5 & & \\
\hline 1.3 & L88 & $\# 1.2$ & $\mathrm{~V}$ & R5 & W15 & D3.0 & & \\
\hline 1.4 & L43 & $\# 1.3$ & V & R1 & W15 & D2. 0 & & \\
\hline 1.5 & L42 & \#1. 4 & $\mathrm{~V}$ & R1 & W15 & D1.9 & B5 & \\
\hline 1.6 & L27 & $\# 1.2$ & A59 & - & W55 & D1. 5 & & \\
\hline 1.7 & L31 & \#1. 6 & $\mathrm{~V}$ & & & D1. 5 & & \\
\hline $1.7 a$ & L5 & \#1. 7 & A10 & + & w4 2 & D0. 5 & B1 & \\
\hline 1.8 & L65 & $\# 1.3$ & A85 & - & W50 & D1.9 & & \\
\hline 1.9 & L43 & \#1.8 & A22 & - & W2 5 & $\mathrm{D} 1.4$ & B5 & \\
\hline 1.10 & L38 & \#1.4 & A15 & + & W4 5 & $\mathrm{D} 1.4$ & B5 & \\
\hline 1.11 & L38 & $\# 1.3$ & A87 & & W60 & D1. 6 & & \\
\hline 1.12 & L24 & $\# 1.11$ & A7 & R2 & W4 5 & D1.0 & B4 & \\
\hline 1.13 & L37 & \#1.2 & A68 & + & W70 & D1. 5 & & \\
\hline 1.14 & L12 & $\# 1.13$ & V & & & D1. 5 & & \\
\hline 1.15 & L7 & $\# 1.13$ & A25 & - & W4 5 & D 0.7 & B2 & \\
\hline 2.1 & L18 & \#Е2 & A2 5 & R3 & W30 & $\mathrm{D} 1.3$ & & <Broken tip> \\
\hline 3.1 & L52 & \#Е3 & A4 & R5 & W30 & D7. 5 & & \\
\hline 3.2 & L37 & \#3.1 & $\mathrm{V}$ & R1 & W2 0 & D5. 0 & & \\
\hline 3.3 & L93 & \#3.2 & $\mathrm{V}$ & & & D4 . 6 & & \\
\hline 3.4 & L112 & \#3.3 & V & R3 & W10 & D3 .6 & & \\
\hline 3.5 & L98 & \#3. 4 & $\mathrm{~V}$ & & & D3. 3 & B10 & \\
\hline 3.6 & L4 0 & \#3.3 & A78 & R1 & W70 & D1. 5 & & \\
\hline 3.7 & L8 & \#3. 6 & $\mathrm{~V}$ & & & D1. 5 & B3 & \\
\hline 3.8 & L93 & \#3.3 & A90 & - & W4 5 & D2. 5 & & \\
\hline
\end{tabular}




\begin{tabular}{|c|c|c|c|c|c|c|c|c|}
\hline 3.9 & L60 & \#3.8 & V & & & D2. 4 & B9 & \\
\hline 3.10 & L38 & \#3. 4 & A95 & R8 & W60 & D1. 6 & B6 & \\
\hline 3.11 & L68 & \#3. 4 & A82 & R4 & W60 & D2. 0 & B7 & \\
\hline 3.12 & L71 & $\# 3.3$ & A8 7 & + & W50 & D1.9 & & \\
\hline 3.13 & L39 & $\# 3.12$ & V & & & D1.9 & B7 & \\
\hline 3.14 & L23 & $\# 3.3$ & A64 & R5 & W60 & $\mathrm{D} 1.4$ & & \\
\hline 3.15 & L21 & $\# 3.14$ & V & & & $\mathrm{D} 1.3$ & B4 & \\
\hline 3.16 & L43 & $\# 3.1$ & A4 6 & + & W60 & D5.0 & & \\
\hline 3.17 & L78 & $\# 3.16$ & V & R1 & W15 & D5.0 & & \\
\hline 3.18 & L127 & $\# 3.17$ & V & R4 & W10 & D3. 8 & & \\
\hline 3.19 & L149 & $\# 3.18$ & V & & & D3. 8 & B11 & \\
\hline 3.20 & L70 & $\# 3.17$ & A71 & R8 & W70 & D2. 4 & & \\
\hline 3.21 & L42 & $\# 3.20$ & V & & & D2. 1 & B8 & \\
\hline 3.22 & L85 & $\# 3.17$ & A76 & - & W50 & D2. 8 & & \\
\hline 3.23 & L62 & $\# 3.22$ & V & & & D2. 2 & B10 & \\
\hline 3.24 & L90 & $\# 3.17$ & A74 & + & W4 5 & D2. 5 & & \\
\hline 3.25 & L49 & $\# 3.24$ & V & & & D2. 1 & B8 & \\
\hline 4.1 & L58 & \#E3 & A10 & R8 & W55 & D7.2 & & \\
\hline 4.2 & L45 & \#4.1 & V & R2 & W50 & D5.4 & & \\
\hline 4.3 & L38 & $\# 4.2$ & V & & & D1. 3 & & $<$ Broken tip $>$ \\
\hline 4.4 & L40 & \#4.1 & A50 & R6 & W30 & $d 3.5$ & & \\
\hline 4.5 & L25 & \#4.4 & $\mathrm{V}$ & R5 & W20 & D2. 5 & & \\
\hline 4.6 & L75 & \#4.5 & $\mathrm{V}$ & R1 & W2 5 & $\mathrm{D} 2.3$ & & \\
\hline 4.7 & L60 & \#4.6 & $\mathrm{V}$ & & & $\mathrm{D} 2.1$ & B10 & \\
\hline 4.8 & L30 & \#4.4 & A4 0 & - & W4 0 & D2. 0 & & \\
\hline 4.9 & L32 & \#4.8 & $\mathrm{V}$ & & & $\mathrm{D} 2.0$ & & \\
\hline 4.10 & L40 & \#4.9 & $\mathrm{V}$ & & & D2. 0 & B6 & \\
\hline 4.11 & L38 & \#4.4 & A4 0 & + & W4 0 & $\mathrm{D} 1.3$ & & \\
\hline 4.12 & L14 & \#4.11 & $\mathrm{V}$ & & & $\mathrm{D} 1.2$ & & \\
\hline 4.13 & L4 & $\# 4.12$ & $\mathrm{~V}$ & & & $\mathrm{D} 1.1$ & B1 & \\
\hline 4.14 & L34 & \#4.2 & A4 2 & R4 & W4 5 & D1. 8 & & \\
\hline 4.15 & L60 & $\# 4.14$ & $\mathrm{~V}$ & & & D1. 7 & & \\
\hline 4.16 & L20 & $\# 4.15$ & $\mathrm{~V}$ & & & D1. 5 & B7 & \\
\hline 4.17 & L75 & $\# 4.2$ & A4 5 & R5 & W30 & D5.0 & & \\
\hline 4.18 & L129 & \#4.17 & $\mathrm{V}$ & & & D3 .6 & & \\
\hline 4.19 & L97 & $\# 4.18$ & $\mathrm{~V}$ & R1 & W10 & D3 .2 & B11 & \\
\hline 4.20 & L93 & \#4.17 & A73 & - & W4 0 & D3 .2 & & \\
\hline 4.21 & L76 & $\# 4.20$ & $\mathrm{~V}$ & & & D3. 0 & B10 & \\
\hline 4.22 & L2 8 & $\# 4.18$ & A76 & R8 & W60 & $\mathrm{D} 1.4$ & B6 & \\
\hline 4.23 & L44 & $\# 4.18$ & A124 & R5 & W90 & D1.9 & B2 & \\
\hline 4.24 & L43 & $\# 4.18$ & A94 & + & W60 & $\mathrm{D} 1.6$ & B4 & \\
\hline 4.25 & L117 & \#4.17 & A68 & + & W4 5 & D2. 6 & & $<$ Broken tip $>$ \\
\hline 4.26 & L18 & $\# 4.25$ & A70 & - & W4 0 & $\mathrm{D} 1.0$ & B1 & \\
\hline 4.27 & L60 & $\# 4.25$ & A105 & - & W30 & $\mathrm{D} 1.7$ & B6 & \\
\hline 4.28 & L16 & $\# 4.25$ & A108 & + & W4 0 & $\mathrm{D} 1.0$ & B3 & \\
\hline 4.29 & L12 & $\# 4.25$ & A82 & + & W45 & D0. 8 & B2 & \\
\hline 4.30 & L38 & $\# 4.2$ & A4 5 & R1 & W60 & $\mathrm{D} 1.4$ & & \\
\hline 4.31 & L6 & $\# 4.30$ & $\mathrm{~V}$ & & & $\mathrm{D} 1.4$ & & \\
\hline 4.32 & L20 & $\# 4.31$ & A13 & + & W4 5 & D0. 8 & B1 & \\
\hline 4.33 & L13 & $\# 4.2$ & A39 & R8 & W60 & $\mathrm{D} 1.3$ & & \\
\hline 4.34 & L14 & $\# 4.33$ & V & & & $\mathrm{D} 1.3$ & & \\
\hline 4.35 & L8 & $\# 4.34$ & $\mathrm{~V}$ & & & $\mathrm{D} 1.3$ & B2 & \\
\hline 4.36 & L70 & $\# 4.1$ & $\mathrm{~A} 2$ & R1 & W95 & D4. 0 & & \\
\hline 4.37 & L5 & $\# 4.36$ & V & & & $\mathrm{D} 1.5$ & & $<$ Broken tip $>$ \\
\hline 4.38 & L45 & $\# 4.36$ & A70 & R5 & W4 0 & D4. 0 & & \\
\hline 4.39 & L2 8 & $\# 4.38$ & $\mathrm{~V}$ & & & $\mathrm{D} 1.2$ & & \\
\hline 4.40 & L50 & $\# 4.38$ & A4 0 & R8 & W60 & D2. 0 & & \\
\hline 4.41 & L4 8 & $\# 4.40$ & $\mathrm{~V}$ & & & $\mathrm{D} 1.8$ & & \\
\hline $4.41 a$ & L15 & \#4.41 & V & & & $\mathrm{D} 1.7$ & B6 & \\
\hline 4.42 & L18 & $\# 4.40$ & A 46 & $\mathrm{R} 2$ & W60 & Do. 9 & & \\
\hline $4.42 \mathrm{a}$ & L5 & $\# 4.42$ & $\mathrm{~V}$ & & & D0.9 & B1 & \\
\hline 4.43 & L50 & $\# 4.38$ & A4 5 & + & W4 5 & D3.0 & & \\
\hline 4.44 & L80 & $\# 4.43$ & $\mathrm{~V}$ & & & D2. 6 & & \\
\hline 4.45 & L49 & \#4.44 & $\mathrm{V}$ & & & $\mathrm{D} 2.2$ & B8 & \\
\hline 4.46 & L50 & $\# 4.38$ & A4 5 & - & W2 0 & $\mathrm{D} 2.2$ & & \\
\hline 4.47 & L83 & $\# 4.46$ & V & & & $\mathrm{D} 2.0$ & & <broken tip> \\
\hline 4.48 & L2 8 & $\# 4.47$ & A71 & - & W60 & $\mathrm{D} 1.2$ & B5 & \\
\hline
\end{tabular}




$\begin{array}{lllllll}4.49 & \text { L33 } & \# 4.47 \text { A80 } & \text { R1 } & \text { W60 } & \text { D1.4 } & \text { B4 } \\ 4.50 & \text { L45 } & \# 4.47 \text { A79 } & + & \text { W45 } & \text { D1.6 } & \text { B5 }\end{array}$

ejf:

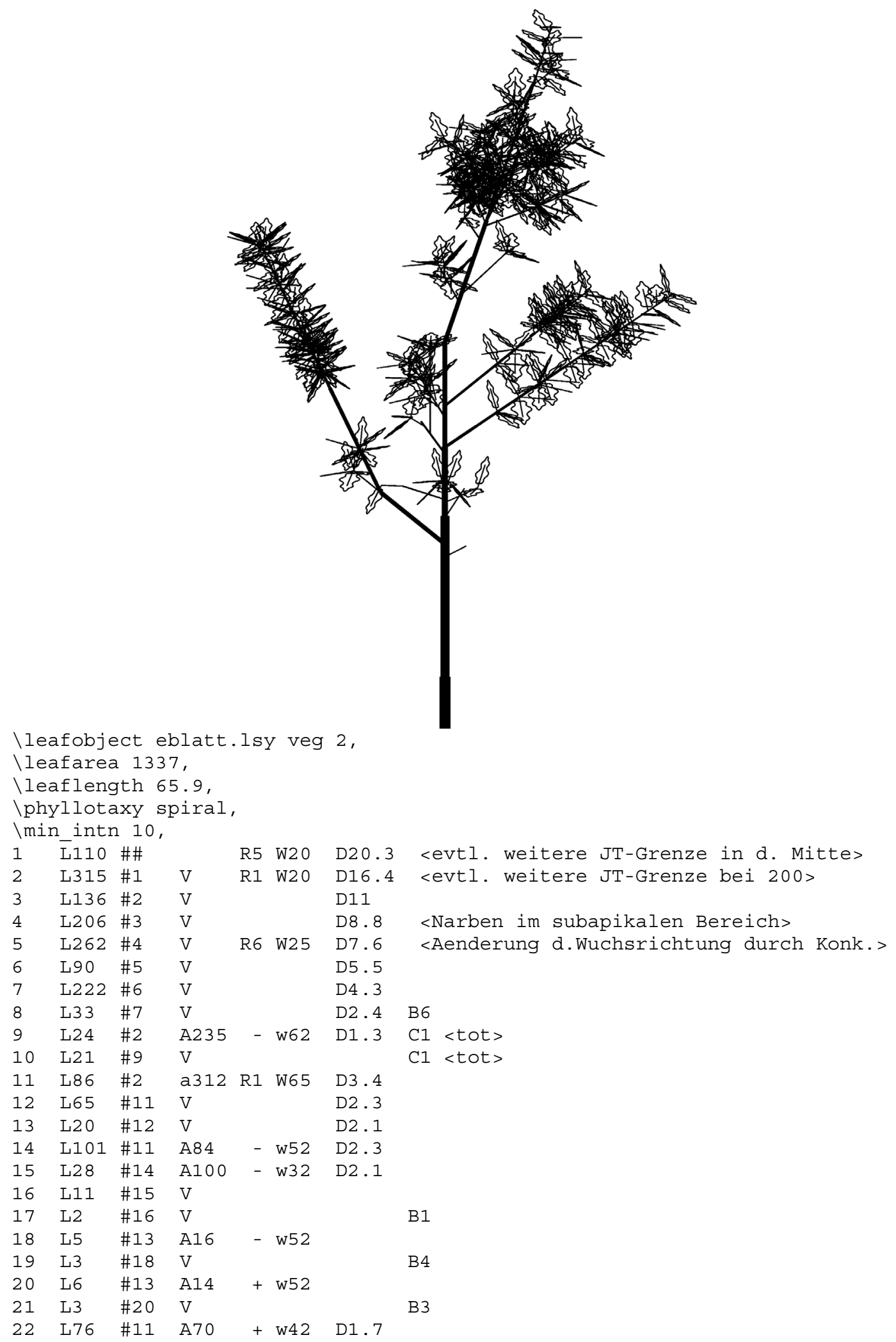




\begin{tabular}{|c|c|c|c|c|c|c|}
\hline L14 & $\# 22$ & V & & & $\mathrm{D} 1.6$ & \\
\hline 24 & $\# 23$ & V & & & & B2 \\
\hline L73 & \#3 & A135 & R7 & W55 & D5. 2 & \\
\hline L201 & \#25 & V & & & D4 . 4 & \\
\hline L95 & $\# 26$ & V & & & D 3.2 & \\
\hline L58 & $\# 27$ & V & & & D3. 0 & \\
\hline L98 & $\# 28$ & V & & & $\mathrm{D} 2.3$ & B8 \\
\hline L4 5 & $\# 26$ & A106 & - & w62 & D1. 7 & \\
\hline L13 & \#30 & V & & & & \\
\hline L21 & \#31 & V & & & & B5 \\
\hline L4 9 & $\# 26$ & A196 & - & w52 & D1. 9 & \\
\hline L22 & \#33 & V & & & & \\
\hline 35 & \#34 & V & & & & B1 \\
\hline L15 & \#34 & A10 & + & w42 & & B4 \\
\hline L2 8 & $\# 27$ & A81 & - & w52 & D1. 5 & \\
\hline L40 & \#37 & V & & & D1. 5 & B7 \\
\hline L2 0 & $\# 28$ & A34 & - & w62 & $\mathrm{D} 1.1$ & B3 \\
\hline L14 & $\# 28$ & A52 & - & w52 & & B3 \\
\hline L19 & $\# 28$ & A4 4 & + & w52 & $\mathrm{D} 1.2$ & B2 \\
\hline L14 & $\# 28$ & A2 5 & + & w62 & $\mathrm{D} 1.3$ & B3 \\
\hline L57 & $\# 26$ & A2 01 & + & w4 2 & D2. 0 & \\
\hline L30 & $\# 43$ & V & & & D1. 9 & \\
\hline L32 & $\# 44$ & V & & & D1. 9 & B5 \\
\hline L35 & $\# 26$ & A186 & + & w52 & D1. 7 & \\
\hline L6 & $\# 46$ & V & & & & \\
\hline 48 & $\# 47$ & V & & & & B3 \\
\hline L13 & $\# 26$ & A136 & + & w52 & $\mathrm{D} 1.4$ & \\
\hline L8 & $\# 49$ & V & & & $\mathrm{d} 1.2$ & \\
\hline L14 & \#50 & V & & & $\mathrm{D} 1.1$ & B4 \\
\hline L27 & \#26 & A80 & + & w62 & $\mathrm{D} 1.3$ & \\
\hline L3 & \#52 & V & & & & \\
\hline L9 & \#53 & V & & & & B3 \\
\hline L80 & $\# 25$ & A50 & + & w52 & D2. 1 & \\
\hline L33 & \#55 & V & & & D1. 9 & \\
\hline L5 & \#56 & V & & & & $\mathrm{C} 1<$ tot $>$ \\
\hline L4 & \#56 & A19 & - & w42 & & \\
\hline L6 & \#58 & V & & & & B3 \\
\hline L80 & \#4 & A5 7 & R2 & W4 0 & D2. 6 & \\
\hline L39 & $\# 60$ & V & & & D2. 0 & \\
\hline L10 & \#61 & V & & & D2. 0 & \\
\hline L10 & \#62 & V & & & D2. 0 & B5 \\
\hline L29 & $\# 60$ & A74 & - & w52 & D1. 7 & \\
\hline L9 & $\# 64$ & V & & & $\mathrm{d} 1.7$ & \\
\hline L5 & \#65 & V & & & D2. 1 & B5 \\
\hline L17 & \#60 & A79 & + & w62 & $\mathrm{D} 1.4$ & \\
\hline L7 & \#67 & V & & & & \\
\hline L5 & \#68 & $\mathrm{V}$ & & & & B4 \\
\hline L169 & \#4 & A8 0 & R7 & W50 & D 4.2 & $<$ Broken tip $>$ \\
\hline L78 & \#70 & A162 & - & w32 & D2. 9 & \\
\hline L65 & \#71 & V & & & $\mathrm{D} 2.8$ & \\
\hline L85 & \#72 & V & & & $\mathrm{D} 1.9$ & B7 \\
\hline L72 & \#72 & A62 & - & w52 & $\mathrm{D} 1.7$ & B6 \\
\hline L2 7 & \#72 & A62 & + & w42 & $\mathrm{D} 1.4$ & B4 \\
\hline L16 & \#72 & A50 & + & w52 & $\mathrm{D} 1.2$ & B4 \\
\hline L26 & \#72 & A29 & + & w52 & $\mathrm{D} 1.3$ & B5 \\
\hline L43 & \#70 & A169 & + & w32 & $\mathrm{D} 2.2$ & \\
\hline L30 & \#78 & $\mathrm{V}$ & & & D2. 1 & \\
\hline L30 & \#79 & $\mathrm{V}$ & & & $\mathrm{D} 2.3$ & B6 \\
\hline L29 & \#70 & A168 & + & w4 2 & D2. 1 & \\
\hline L35 & \#81 & $\mathrm{V}$ & & & D2. 0 & \\
\hline L43 & \#82 & $\mathrm{V}$ & & & D1.9 & B7 \\
\hline L4 7 & \#70 & A165 & + & w72 & D1.9 & $<$ Broken tip $>$ \\
\hline L6 & \#84 & A37 & + & w32 & d1. 9 & \\
\hline L12 & \#85 & $\mathrm{V}$ & & & D1.9 & B5 \\
\hline L33 & \#70 & A103 & + & w52 & D1. 5 & $<$ Broken tip $>$ \\
\hline L4 & \#87 & A30 & - & w22 & & \\
\hline L6 & \#88 & V & & & & B3 \\
\hline
\end{tabular}




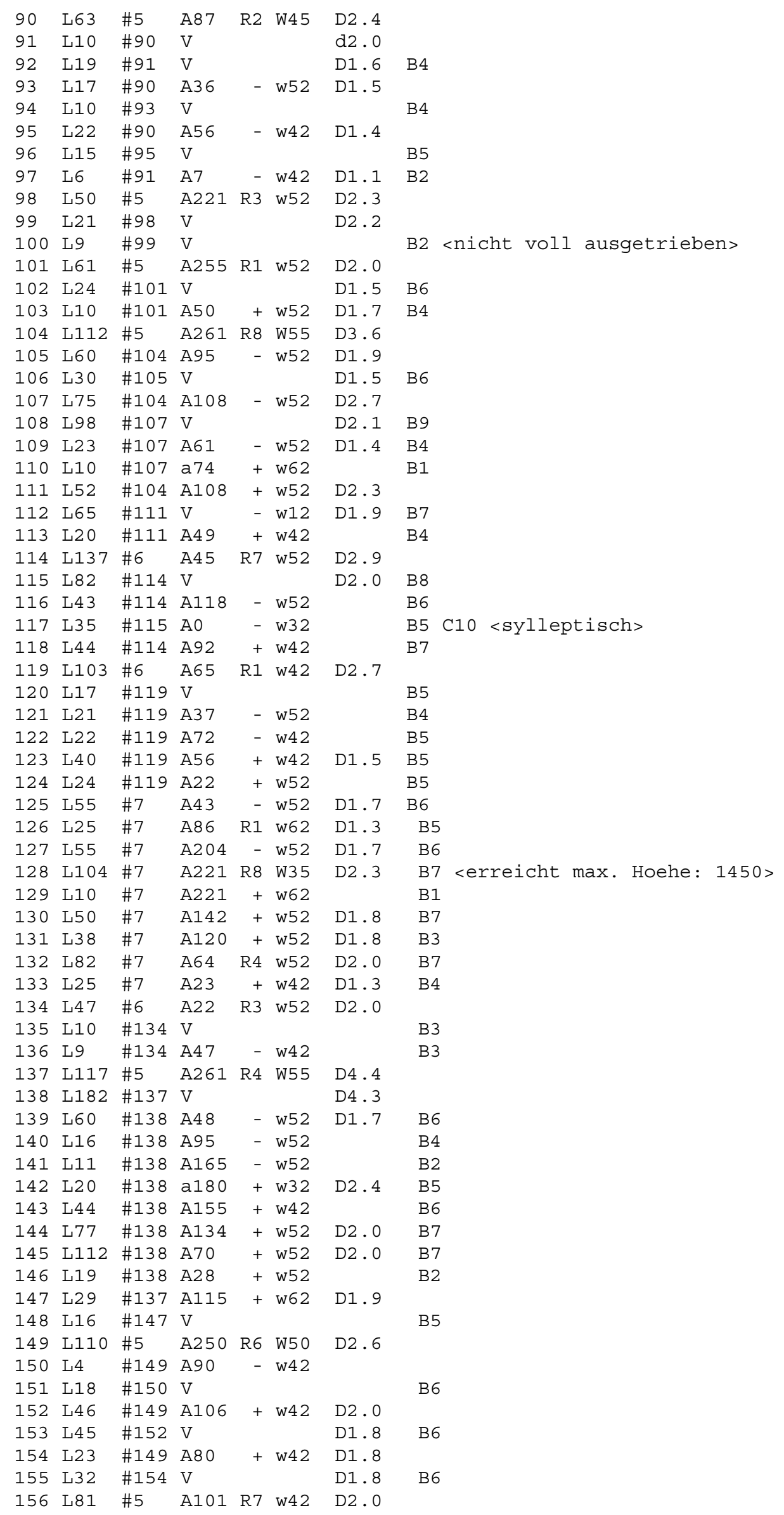




\begin{tabular}{|c|c|c|c|c|c|c|c|}
\hline 157 & L26 & \#156 & $\mathrm{V}$ & & & D1. 8 & \\
\hline 158 & L23 & \#157 & $\mathrm{V}$ & & & D1. 9 & B5 \\
\hline 159 & L90 & \#3 & A122 & $\mathrm{R} 4$ & W4 5 & D3. 1 & \\
\hline 160 & L104 & \#159 & A5 6 & $\mathrm{R} 1$ & W45 & D2. 6 & \\
\hline 161 & L2 7 & \#160 & $\mathrm{V}$ & & & $\mathrm{d} 2.4$ & \\
\hline 162 & L15 & \#161 & $\mathrm{V}$ & & & $\mathrm{d} 2.2$ & \\
\hline 163 & L29 & \#162 & $\mathrm{V}$ & & & D2. 1 & B6 \\
\hline 164 & L8 & \#160 & A95 & + & w52 & d1. 5 & \\
\hline 165 & L6 & \#164 & $\mathrm{V}$ & & & d1 .4 & \\
\hline 166 & L10 & \#165 & $\mathrm{V}$ & & & D1. 4 & B4 \\
\hline 167 & L6 & $\# 160$ & A61 & + & w52 & $\mathrm{d} 1.6$ & \\
\hline 168 & L6 & \#167 & $\mathrm{V}$ & & & $\mathrm{d} 1.6$ & \\
\hline 169 & L14 & \#168 & $\mathrm{V}$ & & & D1. 6 & B5 \\
\hline 170 & L12 & \#159 & A76 & + & w42 & D1. 5 & \\
\hline 171 & L12 & \#170 & $\mathrm{V}$ & & & D1. 4 & \\
\hline 172 & L3 & \#171 & $\mathrm{V}$ & & & & \\
\hline 173 & L5 & \#172 & $\mathrm{V}$ & & & & B3 \\
\hline 174 & L55 & $\# 2$ & a312 & R6 & w42 & D1. 5 & $\mathrm{Cl}<$ tot $>$ \\
\hline 175 & L22 & \#174 & $\mathrm{V}$ & & & & $\mathrm{Cl}<$ tot $>$ \\
\hline 176 & L14 & \#174 & a 42 & & & & $\mathrm{C} 1<$ tot $>$ \\
\hline 177 & L158 & $\# 2$ & A2 60 & R3 & W50 & D7. 6 & \\
\hline 178 & L225 & \#177 & $\mathrm{V}$ & $\mathrm{R} 1$ & W25 & D6. 5 & $<$ Narbe an der Basis $>$ \\
\hline 179 & L110 & \#178 & $\mathrm{V}$ & & & D5. 0 & \\
\hline 180 & L168 & \#179 & $\mathrm{V}$ & & & D3. 8 & \\
\hline 181 & L62 & \#177 & A60 & - & w4 2 & D1. 8 & \\
\hline 182 & L21 & \#181 & $\mathrm{V}$ & & & D1. 5 & \\
\hline 183 & L22 & \#182 & $\mathrm{V}$ & & & & $\mathrm{C} 1<$ tot $>$ \\
\hline 184 & L89 & \#177 & A100 & - & w42 & D2 .3 & \\
\hline 185 & L39 & \#184 & $\mathrm{V}$ & & & $\mathrm{D} 2.0$ & \\
\hline 186 & L31 & \#185 & $\mathrm{V}$ & & & D1. 9 & \\
\hline 187 & L9 & \#186 & $\mathrm{V}$ & & & & B5 \\
\hline 188 & L33 & \#178 & A64 & - & w62 & D1. 7 & \\
\hline 189 & L12 & \#188 & $\mathrm{V}$ & & & D1. 7 & \\
\hline 190 & L24 & \#189 & $\mathrm{V}$ & & & D1. 5 & B7 \\
\hline 191 & L51 & \#178 & A2 10 & - & w52 & D1. 9 & \\
\hline 192 & L24 & \#191 & $\mathrm{V}$ & & & D1. 9 & \\
\hline 193 & L18 & \#192 & $\mathrm{V}$ & & & D1. 9 & B4 \\
\hline 194 & L82 & \#178 & a223 & - & w42 & D2 .3 & <Spitze abgebrochen> \\
\hline 195 & L4 8 & \#194 & A68 & - & w4 2 & D1. 8 & \\
\hline 196 & L15 & \#195 & $\mathrm{V}$ & & & D1. 4 & B5 \\
\hline 197 & L29 & \#194 & A76 & + & w42 & & $\mathrm{C} 1<$ tot $>$ \\
\hline 198 & L4 7 & \#179 & A99 & - & w52 & D2. 0 & \\
\hline 199 & L35 & \#198 & A4 0 & - & w32 & D1 . 4 & B5 \\
\hline 200 & L10 & \#198 & A33 & + & w52 & D1. 2 & B3 \\
\hline 201 & L70 & \#180 & A31 & - & w52 & D1. 5 & B6 \\
\hline 202 & L45 & \#180 & A97 & - & w62 & D1. 6 & B5 \\
\hline 203 & L76 & \#180 & A160 & - & w52 & D2. 0 & B7 \\
\hline 204 & L45 & \#180 & A166 & + & w32 & D2. 1 & B5 \\
\hline 205 & L117 & \#180 & A124 & + & w52 & D2. 1 & B8 \\
\hline 206 & L90 & \#179 & A109 & + & w42 & D2 .4 & \\
\hline 207 & L110 & $\# 206$ & $\mathrm{~V}$ & & & D2. 1 & B8 \\
\hline 208 & L30 & $\# 206$ & A70 & + & w52 & D1. 2 & B3 \\
\hline 209 & L40 & \#179 & A51 & + & w52 & D1. 7 & \\
\hline 210 & L30 & \#209 & $\mathrm{V}$ & & & D1. 5 & B5 \\
\hline 211 & L75 & \#178 & A22 0 & + & w42 & D2. 5 & \\
\hline 212 & L22 & \#211 & A65 & - & w62 & D1. 4 & \\
\hline 213 & L60 & \#211 & A54 & + & w32 & D2. 3 & \\
\hline 214 & L90 & $\# 213$ & $\mathrm{~V}$ & & & D1. 9 & B8 \\
\hline 215 & L25 & $\# 213$ & A5 8 & + & w52 & D1. 4 & B3 \\
\hline 216 & L80 & \#178 & A188 & + & w52 & D2. 7 & \\
\hline 217 & L80 & \#216 & V & & & D2. 8 & \\
\hline 218 & L91 & \#217 & $\mathrm{V}$ & & & D1. 9 & B7 \\
\hline 219 & L58 & \#217 & A5 8 & - & w62 & D1. 7 & B6 \\
\hline 220 & L21 & \#217 & A74 & - & w42 & D1. 2 & B4 \\
\hline 221 & L100 & $\# 217$ & A 67 & + & w52 & $\mathrm{D} 1.6$ & B5 \\
\hline
\end{tabular}


ejg:

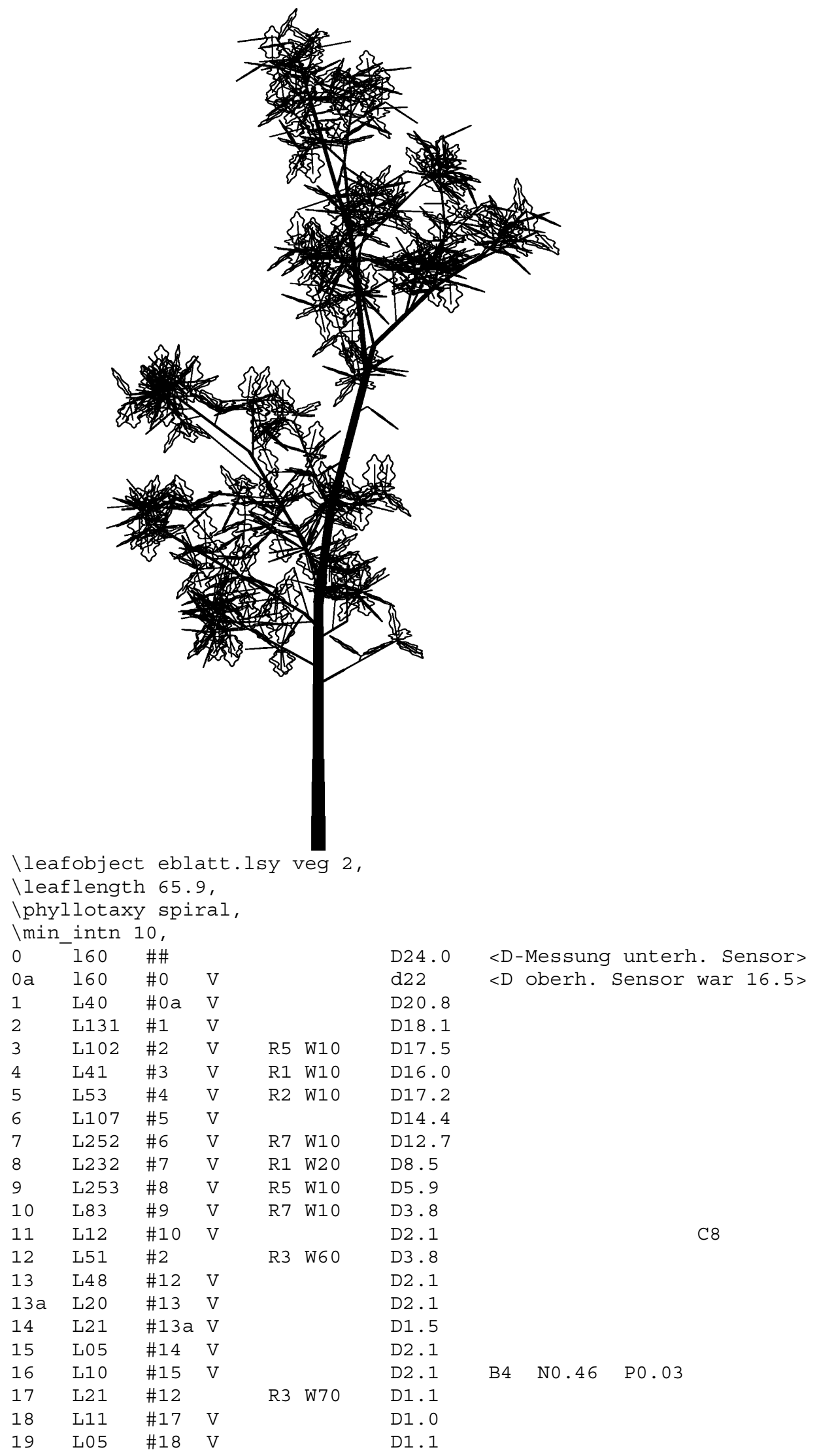




\begin{tabular}{|c|c|c|c|c|c|c|c|c|c|}
\hline 20 & L10 & \#13 & A2 8 & $\mathrm{R} 2$ & W50 & D1. 1 & & & \\
\hline 21 & L02 & \#20 & $\mathrm{V}$ & & & D1. 1 & B2 & N0.06 & P0.01 \\
\hline 22 & L64 & \#3 & A30 & R7 & W60 & D3. 4 & & & \\
\hline 23 & L72 & \#22 & $\mathrm{V}$ & & & D2. 8 & & & \\
\hline 24 & L 67 & $\# 23$ & $\mathrm{~V}$ & & & D2. 2 & & & \\
\hline 25 & L05 & $\# 24$ & $\mathrm{~V}$ & $\mathrm{R} 8$ & W60 & D2. 4 & & & \\
\hline 26 & L03 & \#25 & $\mathrm{V}$ & & & D2. 8 & B5 & No .20 & P0.01 \\
\hline 27 & L1 7 & $\# 22$ & A4 9 & R7 & W60 & D1. 4 & & & \\
\hline 28 & L20 & \#27 & $\mathrm{V}$ & & & $\mathrm{D} 1.2$ & & & \\
\hline 29 & L02 & \#28 & $\mathrm{V}$ & & & $\mathrm{D} 1.2$ & B2 & No. 06 & P0.01 \\
\hline 30 & L08 & \#28 & & $\mathrm{R} 8$ & W30 & D1. 0 & B3 & No. 11 & P0.01 \\
\hline 31 & L4 7 & $\# 23$ & & $\mathrm{R} 4$ & W4 5 & D1. 5 & & & \\
\hline 32 & L12 & \#31 & $\mathrm{V}$ & & & D1. 5 & & & \\
\hline 33 & L18 & \#32 & $\mathrm{V}$ & & & D1. 8 & B6 & No .37 & P0.03 \\
\hline 34 & L22 & $\# 24$ & A4 1 & R6 & W55 & D1. 4 & & & \\
\hline 35 & L15 & \#34 & $\mathrm{V}$ & & & D1. 3 & B5 & No .19 & P0. 02 \\
\hline 36 & L08 & \#34 & & R3 & W55 & D0. 5 & B2 & No . 04 & P0.01 \\
\hline 37 & L06 & $\# 24$ & & $\mathrm{R} 2$ & W2 0 & D1. 8 & B2 & No .12 & P0.01 \\
\hline 38 & L10 & \#24 & A5 5 & R1 & W50 & D1. 2 & & & \\
\hline 39 & L2 8 & $\# 24$ & A32 & R3 & W55 & D1. 5 & & & \\
\hline 40 & L22 & \#39 & $\mathrm{V}$ & & & D1. 3 & B5 & No .34 & P0.03 \\
\hline 41 & L12 & $\# 24$ & A12 & $\mathrm{R} 2$ & W70 & D1. 4 & & & \\
\hline 42 & L04 & $\# 41$ & $\mathrm{~V}$ & & & D1. 0 & B2 & N0. 06 & P0.01 \\
\hline 43 & L8 0 & \#3 & A81 & $\mathrm{R} 4$ & W60 & D3. 3 & & & \\
\hline 44 & $\mathrm{~L} 42$ & $\# 43$ & $\mathrm{~V}$ & R7 & W4 0 & D3. 0 & & & \\
\hline 45 & L99 & $\# 44$ & $\mathrm{~V}$ & R1 & W10 & D2. 5 & & & \\
\hline 46 & L2 0 & $\# 45$ & $\mathrm{~V}$ & R1 & W50 & D1. 7 & & & \\
\hline 47 & L10 & $\# 46$ & $\mathrm{~V}$ & & & D1. 5 & B7 & No .49 & P0. 04 \\
\hline 48 & L4 7 & $\# 43$ & A39 & R1 & W60 & D1. 4 & & & \\
\hline 49 & L09 & $\# 48$ & $\mathrm{~V}$ & & & D1. 3 & B6 & No .30 & P0.01 \\
\hline 50 & L2 5 & $\# 45$ & A85 & $\mathrm{R} 4$ & W4 5 & D2. 0 & & & \\
\hline 51 & L11 & \#50 & $\mathrm{V}$ & & & D1. 8 & B5 & No .47 & P0. 04 \\
\hline 52 & L24 & $\# 45$ & & R5 & W10 & D1. 8 & & & \\
\hline 53 & L03 & \#52 & A2 0 & $\mathrm{R} 8$ & W60 & D1. 1 & B4 & No. 16 & P0.01 \\
\hline 54 & L34 & $\# 45$ & A72 & $\mathrm{R} 4$ & W4 5 & D1. 6 & & & \\
\hline 55 & L24 & $\# 54$ & $\mathrm{~V}$ & R1 & W4 0 & D1. 6 & B6 & N0. 55 & P0. 06 \\
\hline 56 & L4 0 & \#3 & & R8 & W60 & D5. 3 & & & \\
\hline 57 & L126 & $\# 56$ & $\mathrm{~V}$ & R3 & W30 & D4. 3 & & & \\
\hline 58 & L163 & \#57 & $\mathrm{V}$ & & & D4. 3 & & & \\
\hline 59 & L4 3 & \#58 & $\mathrm{V}$ & $\mathrm{R} 2$ & W4 5 & D2. 1 & & & \\
\hline 60 & L8 6 & \#56 & A15 & R5 & W4 0 & D2. 1 & & & \\
\hline 61 & L6 8 & \#60 & $\mathrm{V}$ & & & D2. 0 & & & \\
\hline 62 & L23 & \#61 & $\mathrm{V}$ & R5 & W10 & D1. 5 & & & \\
\hline 63 & L15 & $\# 62$ & $\mathrm{~V}$ & & & D1. 8 & B6 & N0. 39 & P0. 02 \\
\hline 64 & L17 & $\# 61$ & A56 & R7 & W55 & D1. 1 & & & \\
\hline 65 & L05 & \#64 & V & & & D1. 3 & B2 & No .10 & P0.01 \\
\hline 66 & L05 & \#61 & A62 & $\mathrm{R} 2$ & W60 & D1. 8 & & & \\
\hline 67 & L04 & \#66 & $\mathrm{V}$ & & & D1. 3 & B2 & N0. 04 & P0.01 \\
\hline 68 & L06 & \#61 & A34 & $\mathrm{R} 8$ & W60 & D1. 5 & & & \\
\hline 69 & L0 7 & \#68 & $\mathrm{V}$ & & & D1. 0 & B4 & No .10 & P0.01 \\
\hline 70 & L3 2 & \#57 & A32 & R7 & W4 5 & D1. 2 & & & \\
\hline 71 & L03 & \#70 & $\mathrm{V}$ & & & D1. 5 & B1 & N0. 01 & P0.01 \\
\hline 72 & L8 2 & \#57 & A84 & $\mathrm{R} 4$ & W50 & D1. 8 & & & \\
\hline 73 & L12 & \#72 & $\mathrm{V}$ & & & D1. 2 & & & \\
\hline 74 & L06 & \#73 & $\mathrm{V}$ & & & D1. 0 & & & \\
\hline 75 & L09 & \#72 & & $\mathrm{R} 4$ & W85 & D1. 1 & & & \\
\hline 76 & L11 & \#75 & $\mathrm{V}$ & & & D1. 1 & B4 & No .18 & P0. 02 \\
\hline 77 & L05 & \#72 & & R1 & W4 5 & D1. 2 & & & \\
\hline 78 & L01 & \#77 & $\mathrm{V}$ & & & D1. 1 & B2 & N0.03 & P0.01 \\
\hline $78 a$ & L4 1 & \#57 & A106 & R7 & W4 5 & D1. 2 & & & \\
\hline $78 \mathrm{~b}$ & L15 & $\# 78 a$ & $\mathrm{~V}$ & & & D1. 3 & B3 & N0. 06 & P0.01 \\
\hline $78 \mathrm{C}$ & L10 & $\# 78 a$ & A30 & R7 & W4 5 & D1. 3 & & & \\
\hline $78 d$ & L10 & $\# 78 \mathrm{c}$ & $\mathrm{V}$ & & & D1. 2 & B4 & No. 04 & P0.01 \\
\hline 79 & L4 5 & \#58 & A2 8 & R6 & W4 5 & D1. 8 & & & \\
\hline 80 & L15 & \#79 & $\mathrm{V}$ & & & D1. 5 & B5 & No .27 & P0.02 \\
\hline & L4 5 & \#58 & A95 & R8 & W4 5 & D1. 3 & B2 & N0. 03 & P0.05 \\
\hline 82 & L54 & \#58 & A12 7 & R7 & W50 & D2. 1 & & & \\
\hline
\end{tabular}




\begin{tabular}{|c|c|c|c|c|c|c|c|c|c|c|}
\hline 83 & L71 & \#82 & $\mathrm{V}$ & & & $\mathrm{D} 1.5$ & B7 & No. 74 & P0. 25 & $\{$ Blattoberfl. 115\} \\
\hline 84 & L12 & \#82 & A35 & R2 & W20 & $\mathrm{D} 1.2$ & B6 & No. 12 & P0.01 & \\
\hline 85 & L45 & \#82 & A4 2 & R5 & W20 & $\mathrm{D} 1.3$ & B4 & No. 37 & P0.06 & \\
\hline 86 & L15 & \#59 & A34 & R3 & W4 0 & $\mathrm{D} 1.2$ & B5 & No. 34 & P0.04 & \\
\hline 87 & L13 & \#59 & A36 & $\mathrm{R} 8$ & W4 0 & $\mathrm{D} 1.2$ & B4 & No.13 & P0.02 & \\
\hline 88 & L12 & \#59 & A29 & R1 & W30 & $\mathrm{D} 1.1$ & B5 & No. 14 & P0.01 & \\
\hline 89 & L35 & \#59 & A25 & R4 & W45 & $\mathrm{D} 1.1$ & B4 & No. 36 & P0.04 & \\
\hline 90 & L08 & \#58 & A52 & R1 & W45 & $\mathrm{D} 1.8$ & & & & \\
\hline 91 & L05 & \#90 & $\mathrm{V}$ & & & $\mathrm{D} 1.0$ & B4 & N0.12 & P0.01 & \\
\hline 92 & L122 & \#57 & & R2 & W30 & $\mathrm{D} 2.1$ & & & & \\
\hline 93 & L20 & \#92 & $\mathrm{V}$ & & & D1. 6 & & & & \\
\hline 94 & L10 & \#93 & $\mathrm{V}$ & & & $\mathrm{D} 1.6$ & & & & \\
\hline 95 & L02 & \#94 & V & & & D1. 4 & B1 & N0.03 & P0.01 & \\
\hline 96 & L10 & \#92 & A88 & R8 & W60 & D1.0 & & & & \\
\hline 97 & L01 & \#96 & $\mathrm{V}$ & & & D1.0 & & & & \\
\hline 98 & L15 & \#94 & & R8 & W70 & D1. 4 & B5 & N0. 37 & P0.01 & \\
\hline 99 & L10 & \#93 & & R8 & W60 & $\mathrm{D} 2.1$ & & & & \\
\hline 100 & L02 & \#99 & $\mathrm{V}$ & & & D1. 5 & B5 & N0.15 & P0.03 & \\
\hline 101 & L15 & \#92 & A78 & R4 & W55 & $\mathrm{D} 1.2$ & & & & \\
\hline 102 & L03 & \#101 & V & & & D1.1 & B3 & N0.11 & P0.01 & \\
\hline 103 & L39 & \#57 & A98 & R1 & W35 & D1. 5 & & & & \\
\hline 104 & L11 & \#103 & $\mathrm{V}$ & & & D2. 0 & B4 & No. 14 & P0.01 & \\
\hline 105 & L05 & \#103 & A2 6 & R3 & W45 & D1. 3 & & & & \\
\hline 106 & L05 & \#105 & $\mathrm{V}$ & & & $\mathrm{D} 1.2$ & & & & \\
\hline 107 & L17 & \#57 & A4 1 & R3 & W70 & D1. 5 & & & & \\
\hline 108 & L10 & \#107 & $\mathrm{V}$ & & & $\mathrm{D} 1.2$ & B4 & No. 14 & P0.01 & \\
\hline 109 & L76 & \#56 & A25 & R8 & W25 & D2. 0 & & & & \\
\hline 110 & L2 8 & \#109 & $\mathrm{V}$ & & & D1. 4 & & & & \\
\hline 111 & L03 & \#110 & $\mathrm{V}$ & & & D1. 4 & & & & \\
\hline 112 & L11 & \#109 & A50 & R3 & W4 0 & D1.1 & & & & \\
\hline 113 & L19 & \#5 & A13 & R1 & W30 & D3 .8 & & & & \\
\hline 114 & L87 & \#113 & $\mathrm{V}$ & & & D2. 9 & & & & \\
\hline 115 & L114 & \#114 & $\mathrm{V}$ & R7 & W30 & $\mathrm{D} 2.2$ & & & & \\
\hline 116 & ᄂ47 & \#113 & A18 & R6 & W60 & D1.1 & & & & \\
\hline 117 & L13 & \#116 & A4 5 & R7 & w60 & $\mathrm{D} 1.3$ & & & & \\
\hline 118 & L02 & \#117 & $\mathrm{V}$ & & & $\mathrm{D} 1.0$ & B1 & No.01 & P0.03 & \\
\hline 119 & L53 & \#114 & A4 4 & $\mathrm{R} 4$ & W45 & $\mathrm{D} 1.3$ & B5 & No.11 & P0.02 & \\
\hline 120 & L06 & \#119 & A4 1 & R5 & W20 & $\mathrm{D} 1.2$ & & & & \\
\hline 121 & L06 & \#120 & $\mathrm{V}$ & & & D1.1 & B4 & N0.17 & P0.01 & \\
\hline 122 & L89 & \#114 & A89 & R6 & W55 & $\mathrm{D} 2.1$ & & & & \\
\hline 123 & L10 & \#122 & $\mathrm{V}$ & & & $\mathrm{D} 1.8$ & & & & \\
\hline 124 & L10 & \#123 & $\mathrm{V}$ & R7 & W50 & D1. 5 & B3 & No. 27 & P0.03 & \\
\hline 125 & L15 & \#122 & A36 & R6 & W55 & Do. 8 & B2 & No. 02 & P0.02 & \\
\hline 126 & L11 & \#122 & A51 & $\mathrm{R} 2$ & W30 & $\mathrm{D} 1.8$ & B4 & No. 07 & P0.01 & \\
\hline 127 & L10 & \#115 & A68 & $\mathrm{R} 2$ & W45 & $\mathrm{D} 1.2$ & & & & \\
\hline $127 a$ & L01 & \#127 & $\mathrm{V}$ & & & $\mathrm{D} 1.3$ & B3 & No. 12 & P0.01 & \\
\hline 128 & L10 & \#115 & A10 & $\mathrm{R} 2$ & W20 & D1. 5 & B3 & N0.19 & P0.01 & \\
\hline 129 & L10 & \#115 & A78 & R7 & W45 & $\mathrm{D} 2.0$ & & & & \\
\hline 130 & L02 & \#129 & $\mathrm{V}$ & & & D1. 8 & B3 & N0.14 & P0.02 & \\
\hline 131 & L10 & \#115 & A55 & R3 & W45 & $\mathrm{D} 1.2$ & & & & \\
\hline 132 & L02 & \#131 & $\mathrm{V}$ & & & $\mathrm{D} 1.2$ & B2 & No. 03 & P0.01 & \\
\hline 133 & L96 & \#114 & & R1 & W60 & D1. 3 & & & & \\
\hline 134 & L03 & \#133 & A94 & R3 & W4 0 & $\mathrm{D} 1.2$ & B3 & N0.11 & P0.01 & \\
\hline 135 & L63 & \#114 & A63 & R1 & W60 & $\mathrm{D} 1.2$ & & & & \\
\hline 136 & L09 & \#135 & $\mathrm{V}$ & & & $\mathrm{D} 1.2$ & & & & \\
\hline 137 & L08 & \#136 & $\mathrm{V}$ & & & $\mathrm{D} 1.2$ & B3 & No. 19 & P0.01 & \\
\hline 138 & L25 & \#114 & $\mathrm{A} 21$ & $\mathrm{R} 2$ & W60 & $\mathrm{D} 1.2$ & & & & \\
\hline 139 & L08 & \#138 & $\mathrm{V}$ & & & $\mathrm{D} 1.1$ & & & & \\
\hline 140 & L07 & \#139 & $\mathrm{V}$ & R1 & W60 & D0. 8 & B3 & No.07 & P0.01 & \\
\hline 141 & L108 & \#5 & A4 1 & R3 & W4 0 & D5 . 8 & & & & \\
\hline 142 & L192 & \#141 & $\mathrm{V}$ & R3 & W5 & $\mathrm{D} 4.8$ & & & & \\
\hline 143 & L151 & \#142 & $\mathrm{V}$ & R3 & W35 & D3. 7 & & & & \\
\hline 144 & L55 & \#143 & $\mathrm{V}$ & R8 & W4 0 & $\mathrm{D} 2.1$ & & & & \\
\hline 145 & L31 & \#141 & A53 & R7 & W75 & $\mathrm{D} 1.2$ & & & & \\
\hline 146 & L10 & \#145 & $\mathrm{V}$ & & & $\mathrm{D} 1.2$ & & & & \\
\hline 147 & L05 & \#146 & $\mathrm{V}$ & R7 & W45 & Do. 9 & B3 & No. 07 & P0.01 & \\
\hline 148 & L77 & \#141 & & R8 & W65 & D1. 3 & & & & \\
\hline
\end{tabular}




\begin{tabular}{|c|c|c|c|c|c|c|c|c|c|}
\hline 149 & L05 & \#148 & A64 & R7 & W30 & D1. 1 & B1 & No. 02 & P0.01 \\
\hline 150 & L04 & \#148 & A73 & R8 & W30 & $\mathrm{D} 2.0$ & & & \\
\hline 151 & L03 & $\# 150$ & $\mathrm{~V}$ & & & $\mathrm{D} 1.6$ & B5 & No .28 & P0.02 \\
\hline 152 & L05 & \#148 & A69 & R2 & W30 & D1. 1 & B4 & No. 09 & P0.01 \\
\hline 153 & L14 7 & $\# 142$ & A1 77 & R7 & W30 & D3. 0 & & & \\
\hline 154 & L22 & \#153 & A2 2 & R7 & W4 0 & D1. 0 & & & \\
\hline 155 & L06 & \#154 & $\mathrm{V}$ & & & D1. 0 & B2 & N0.03 & P0.01 \\
\hline 156 & L08 & \#153 & A78 & R8 & W6 5 & D1. 3 & & & \\
\hline 157 & L08 & \#156 & V & & & $\mathrm{D} 1.6$ & B4 & No .49 & P0.03 \\
\hline 158 & L17 & \#153 & A 114 & R6 & W30 & D1. 5 & & & \\
\hline 159 & L03 & \#158 & A03 & R5 & W2 0 & D0. 8 & B1 & No. 02 & P0.01 \\
\hline 160 & L04 & \#153 & A1 72 & R7 & W70 & $\mathrm{D} 2.3$ & & & \\
\hline 161 & L38 & \#153 & A101 & R2 & W30 & $\mathrm{D} 2.0$ & & & \\
\hline 162 & L23 & \#161 & A2 0 & R5 & W30 & D1. 5 & B4 & No .33 & P0.05 \\
\hline 163 & L4 5 & \#153 & A60 & R6 & W30 & D1. 5 & & & \\
\hline 164 & L12 & \#153 & $\mathrm{V}$ & & & D1. 3 & B4 & No .28 & P0.02 \\
\hline 165 & L21 & $\# 143$ & A74 & R1 & W70 & $\mathrm{D} 1.3$ & & & \\
\hline 166 & L0 8 & \#165 & $\mathrm{V}$ & & & D1. 3 & B5 & No .27 & P0.03 \\
\hline 167 & L4 3 & \#143 & A121 & $\mathrm{R} 4$ & W50 & D1. 3 & & & \\
\hline 168 & L08 & \#167 & A30 & R3 & W30 & D1. 5 & B4 & No .21 & P0.01 \\
\hline 169 & L6 5 & \#143 & & R3 & W60 & $\mathrm{D} 2.1$ & & & \\
\hline 170 & L18 & \#169 & A 34 & R6 & W30 & D1. 1 & B4 & N0. 19 & P0.01 \\
\hline 171 & L36 & \#169 & A4 0 & R3 & W30 & D1. 2 & B4 & N0. 36 & P0.03 \\
\hline 172 & L18 & \#169 & A2 5 & R6 & W60 & $\mathrm{D} 1.2$ & B5 & No .18 & P0.02 \\
\hline 173 & L4 3 & $\# 144$ & A1 7 & R6 & W4 0 & D1. 8 & B7 & N0. 82 & P0.13 \\
\hline 174 & L2 0 & \#144 & A2 8 & $\mathrm{R} 4$ & W4 0 & D1. 3 & B4 & N0. 35 & P0.04 \\
\hline 175 & L82 & \#143 & & R6 & W3 5 & D2. 1 & & & \\
\hline 176 & L1 7 & \#175 & A16 & R3 & W50 & D1. 1 & B4 & N0. 08 & P0.02 \\
\hline 177 & L06 & \#175 & A3 8 & R1 & W2 5 & D1. 4 & B5 & N0. 06 & P0.01 \\
\hline 178 & L4 6 & \#175 & A4 8 & R5 & W2 5 & D1. 4 & B4 & No .40 & P0.08 \\
\hline 179 & L3 7 & \#175 & A2 0 & R7 & W60 & D1. 1 & B5 & No .28 & P0.04 \\
\hline 180 & L19 & \#143 & A133 & R1 & W50 & D1. 5 & & & \\
\hline $180 a$ & L09 & \#180 & $\mathrm{V}$ & R2 & W60 & D1. 0 & B4 & No .11 & P0.01 \\
\hline 181 & L39 & \#143 & A101 & R6 & W60 & D1. 3 & & & \\
\hline 182 & L0 8 & \#181 & $\mathrm{V}$ & & & D1. 5 & B3 & N0. 08 & P0.01 \\
\hline 183 & L01 & \#182 & A1 7 & R7 & W4 0 & D1. 3 & B1 & N0. 01 & P0.01 \\
\hline 184 & L14 8 & \#142 & A114 & R3 & W30 & D2. 0 & & & \\
\hline 185 & L15 & \#184 & $\mathrm{V}$ & & & D1. 6 & & & \\
\hline 186 & L113 & \#141 & & R3 & W3 5 & D1. 8 & & & \\
\hline 187 & L06 & \#186 & A110 & R8 & W70 & $\mathrm{D} 1.3$ & & & \\
\hline 188 & L04 & \#187 & $\mathrm{V}$ & & & D1. 2 & B1 & N0. 05 & P0.01 \\
\hline 189 & L12 & \#186 & A103 & R3 & W50 & D1. 5 & & & \\
\hline 190 & L13 & \#189 & $\mathrm{V}$ & & & D1. 2 & B5 & No. 30 & P0.01 \\
\hline 191 & L15 & \#186 & A78 & R7 & W60 & Do. 9 & B4 & N0. 15 & P0.01 \\
\hline 192 & L107 & $\# 6$ & A86 & R7 & W60 & D2. 1 & & & \\
\hline 193 & L08 & \#192 & $\mathrm{V}$ & R7 & W15 & $\mathrm{D} 2.1$ & & & \\
\hline 194 & L05 & \#193 & $\mathrm{V}$ & & & D1. 8 & B2 & N0. 15 & P0.01 \\
\hline 195 & L11 & \#192 & A 81 & R8 & W50 & D1. 4 & & & \\
\hline 196 & L03 & \#195 & $\mathrm{V}$ & R7 & W60 & D0. 5 & B3 & No . 04 & P0.01 \\
\hline 197 & L11 & \#192 & & $\mathrm{R} 4$ & W30 & D1. 0 & & & \\
\hline 198 & L15 & \#197 & $\mathrm{V}$ & R1 & W10 & D1. 5 & B4 & No .44 & P0.04 \\
\hline $198 a$ & L06 & \#192 & A 58 & R2 & W55 & D1. 0 & & & \\
\hline 198b & L10 & \#198a & $\mathrm{V}$ & & & D1. 0 & B6 & No .14 & P0.01 \\
\hline 199 & L199 & \#6 & & R3 & W3 5 & D2. 8 & & & \\
\hline 200 & L10 & \#199 & A109 & R1 & W60 & D1. 7 & & & \\
\hline 201 & L11 & $\# 200$ & $\mathrm{~V}$ & R3 & W30 & D1. 1 & B4 & No .20 & P0.01 \\
\hline 202 & L15 & \#199 & & R8 & W4 5 & D1. 8 & B3 & No. 09 & P0.01 \\
\hline 203 & L2 0 & \#199 & & $\mathrm{R} 4$ & W4 5 & $\mathrm{D} 1.4$ & & & \\
\hline 204 & L12 & $\# 203$ & $\mathrm{~V}$ & & & $\mathrm{D} 1.2$ & B4 & No .18 & P0.02 \\
\hline 205 & L07 & \#203 & & R7 & W4 5 & $\mathrm{D} 1.4$ & B3 & N0. 35 & P0.02 \\
\hline 206 & L53 & $\# 7$ & A3 5 & R1 & W65 & D1. 5 & & & \\
\hline 207 & L12 & \#206 & A 50 & R3 & W4 5 & D1. 3 & & & \\
\hline 208 & L06 & \#207 & $\mathrm{V}$ & & & $\mathrm{D} 1.3$ & B3 & No .13 & P0.01 \\
\hline 209 & L07 & $\# 206$ & A39 & R1 & W4 0 & D1. 1 & B3 & No .04 & P0.01 \\
\hline 210 & L152 & $\# 7$ & A133 & R3 & W4 0 & D2. 8 & & & \\
\hline 211 & L15 & \#210 & A14 5 & R8 & W50 & D1. 7 & & & \\
\hline 212 & L07 & \#211 & $\mathrm{V}$ & & & D1. 7 & B5 & No .42 & P0.0I \\
\hline
\end{tabular}




\begin{tabular}{|c|c|c|c|c|c|c|c|c|c|}
\hline 213 & L48 & \#210 & A130 & R4 & W35 & $\mathrm{D} 2.1$ & & & \\
\hline 214 & L22 & \#213 & A21 & R3 & W25 & D1. 5 & B5 & N0. 28 & P0.03 \\
\hline 15 & L06 & \#213 & A42 & R1 & W30 & $\mathrm{D} 1.8$ & B4 & N0. 12 & P0.01 \\
\hline 16 & L21 & \#210 & A62 & R7 & W4 5 & D1.7 & & & \\
\hline 17 & L02 & \#216 & V & & & $\mathrm{D} 1.3$ & B1 & N0.02 & P0.01 \\
\hline 18 & L51 & \#7 & & & & D8. 3 & & & \\
\hline 219 & L161 & \#218 & V & R4 & W4 0 & D7. 1 & & & \\
\hline 220 & L114 & \#219 & V & R6 & W10 & D5.1 & & & \\
\hline 221 & L161 & $\# 220$ & V & R3 & W30 & D 3.2 & B9 & N1. 66 & P0.89 \\
\hline 222 & L167 & \#218 & & R8 & W35 & D 4.2 & & & \\
\hline 223 & L126 & $\# 222$ & V & & & $\mathrm{D} 2.2$ & & & \\
\hline 224 & L83 & $\# 222$ & A75 & R2 & W70 & $\mathrm{D} 1.6$ & & & \\
\hline 225 & L13 & \#224 & A75 & R3 & W30 & D0. 8 & B5 & N0. 10 & P0.01 \\
\hline 226 & L55 & \#224 & A55 & R1 & W50 & D0. 8 & B5 & N0. 12 & P0.01 \\
\hline 227 & L6 7 & $\# 222$ & A140 & R2 & W50 & D2. 1 & & & \\
\hline 228 & L11 & \#227 & A 22 & R8 & W50 & D0. 8 & B4 & N0. 10 & P0.01 \\
\hline 229 & L14 & \#227 & A4 6 & R6 & W35 & $\mathrm{D} 1.1$ & B4 & N0.12 & P0.01 \\
\hline 230 & L09 & $\# 227$ & A58 & R2 & W30 & $\mathrm{D} 1.2$ & B4 & No. 24 & P0.01 \\
\hline 231 & L03 & \#22 7 & A 32 & R3 & W4 0 & D0. 8 & B1 & N0.02 & P0.01 \\
\hline 232 & L145 & \#222 & & R8 & W60 & D2. 5 & & & \\
\hline 233 & L42 & \#232 & A3 7 & R2 & W30 & $\mathrm{D} 1.2$ & B7 & N0. 36 & P0.07 \\
\hline 234 & L22 & \#232 & A106 & R6 & W30 & $\mathrm{D} 1.4$ & B6 & N0.51 & P0.08 \\
\hline 235 & L50 & \#232 & A90 & R3 & W30 & $\mathrm{D} 1.3$ & B6 & N0.41 & P0.03 \\
\hline 236 & L26 & \#232 & A2 0 & R7 & W30 & $\mathrm{D} 1.0$ & B4 & N0.11 & P0.01 \\
\hline 237 & L21 & $\# 223$ & A70 & R3 & W30 & $\mathrm{D} 1.0$ & B3 & No. 16 & P0.01 \\
\hline 238 & L25 & $\# 223$ & A111 & R8 & W35 & D1. 5 & B6 & N0. 50 & P0.05 \\
\hline 239 & L17 & $\# 223$ & A8 7 & R7 & W35 & $\mathrm{D} 1.8$ & B5 & N0. 35 & P0.04 \\
\hline 240 & L05 & $\# 223$ & A45 & R2 & W10 & D0. 5 & B1 & N0.01 & P0.01 \\
\hline 241 & L131 & \#222 & A155 & R4 & W4 0 & D2. 7 & & & \\
\hline 242 & L11 & \#241 & V & & & D2. 0 & B2 & N0.19 & P0.02 \\
\hline 243 & L4 0 & \#241 & A85 & R7 & W50 & D1. 5 & B7 & N0. 54 & P0.09 \\
\hline 244 & L20 & \#241 & A113 & R2 & W4 0 & $\mathrm{D} 1.0$ & B5 & N0.19 & P0.01 \\
\hline 245 & L10 & \#241 & & R3 & W4 5 & D2. 5 & B3 & N0. 29 & P0.05 \\
\hline 246 & L83 & \#219 & A60 & R1 & W55 & $\mathrm{D} 1.8$ & & & \\
\hline 247 & L26 & \#246 & V & & & $\mathrm{D} 1.3$ & B8 & N0. 76 & P0.08 \\
\hline 248 & L113 & \#219 & A95 & R8 & W60 & D2. 9 & & & \\
\hline 249 & L85 & \#248 & V & & & D2. 0 & B11 & N1. 70 & P0.39 \\
\hline 250 & L18 & \#248 & A71 & R8 & W4 5 & $\mathrm{D} 1.2$ & B6 & N0. 16 & P0.01 \\
\hline 251 & L42 & \#248 & A58 & R2 & W4 5 & $\mathrm{D} 1.2$ & B6 & N0. 29 & P0.04 \\
\hline 252 & L20 & \#220 & $\mathrm{A} 20$ & R7 & W8 0 & D0. 9 & B3 & N0.09 & P0. 02 \\
\hline 253 & L21 & $\# 220$ & A2 7 & R1 & W60 & $\mathrm{D} 1.0$ & B6 & N0. 11 & P0. 02 \\
\hline 254 & L131 & \#220 & A76 & R7 & W35 & D3. 2 & B7 & N1.01 & P0. 80 \\
\hline 255 & L19 & \#220 & A104 & R8 & W50 & $\mathrm{D} 1.2$ & B3 & N0. 14 & P0.01 \\
\hline 256 & L65 & \#220 & & R1 & W15 & D2. 0 & B7 & No .64 & P0.16 \\
\hline 257 & L122 & \#219 & & $\mathrm{R} 2$ & W30 & D 4.1 & & & \\
\hline 258 & L166 & \#257 & V & R1 & W2 0 & D3. 1 & B8 & N1. 61 & P1.06 \\
\hline 259 & L31 & \#257 & A23 & R8 & W60 & $\mathrm{D} 1.3$ & B6 & No .22 & P0. 05 \\
\hline 260 & L103 & $\# 257$ & A94 & R6 & W30 & D1.9 & B6 & N0. 56 & P0.23 \\
\hline 261 & L117 & \#219 & A85 & R3 & W4 0 & D3. 5 & & & \\
\hline 262 & L52 & \#261 & V & & & $\mathrm{D} 1.8$ & B9 & N0. 86 & P0.14 \\
\hline 263 & L20 & \#261 & A4 7 & R8 & W55 & D1.1 & B5 & N0. 15 & P0.02 \\
\hline 264 & L43 & $\# 261$ & & R7 & W4 5 & $\mathrm{D} 1.2$ & B4 & N0. 25 & P0.02 \\
\hline 265 & L08 & $\# 261$ & & R3 & W4 5 & $\mathrm{D} 1.0$ & B2 & N0. 13 & P0.01 \\
\hline 266 & L35 & \#8 & A34 & R6 & W60 & D1. 1 & B2 & N0.05 & P0.03 \\
\hline 267 & L45 & \#8 & A77 & R4 & W90 & $\mathrm{D} 1.1$ & & & \\
\hline $267 a$ & L07 & \#267 & $\mathrm{V}$ & & & D1. 0 & B3 & N0. 10 & P0.01 \\
\hline 268 & L52 & \#8 & A125 & R3 & W70 & D1. 6 & & & \\
\hline 269 & L18 & \#268 & $\mathrm{V}$ & & & $\mathrm{D} 1.2$ & B4 & N0. 27 & P0.02 \\
\hline 270 & L04 & \#268 & A4 0 & R8 & W70 & D0. 6 & B1 & N0.01 & P0.01 \\
\hline 271 & L25 & \#268 & A43 & R4 & W4 0 & $\mathrm{D} 1.0$ & B5 & No. 20 & P0.03 \\
\hline 272 & L12 & \#268 & A23 & R2 & W80 & D0. 8 & B4 & N0.07 & P0.01 \\
\hline 273 & L157 & \#8 & A215 & R1 & W50 & D3. 0 & & & \\
\hline 274 & L61 & \#273 & $\mathrm{V}$ & R1 & W10 & D1. 8 & B8 & N0. 96 & P0.18 \\
\hline 275 & L3 7 & \#273 & A81 & R7 & W50 & $\mathrm{D} 1.2$ & B6 & N0.31 & P0.06 \\
\hline 276 & L66 & \#273 & & R6 & W25 & D1.9 & B6 & N0. 40 & P0.13 \\
\hline 277 & L65 & \#273 & & R4 & W25 & D1.9 & B7 & N0. 62 & P0.16 \\
\hline 278 & L10 & \#273 & A71 & R2 & W60 & D0. 5 & B1 & N0.02 & P0.01 \\
\hline
\end{tabular}




\begin{tabular}{|c|c|c|c|c|c|c|c|c|c|}
\hline \multirow{2}{*}{279} & \multirow{2}{*}{$\begin{array}{l}\text { L52 } \\
\text { L161 }\end{array}$} & \multicolumn{2}{|l|}{$\# 8$} & $\mathrm{R} 8$ & W50 & D1 . 4 & \multirow[t]{2}{*}{ B8 } & \multirow[t]{2}{*}{ N0. 31} & \multirow[t]{2}{*}{ P0.08 } \\
\hline & & $\# 8$ & & R7 & W30 & D4 . 7 & & & \\
\hline 281 & L95 & $\# 280$ & $\mathrm{~V}$ & R8 & W15 & D3. 5 & & & \\
\hline 282 & L81 & $\# 280$ & & R4 & W3 5 & D3. 2 & & & \\
\hline 283 & L125 & \#282 & $\mathrm{V}$ & R1 & W5 & D2. 7 & B9 & N0. 83 & P0. 30 \\
\hline 284 & L5 7 & \#282 & A5 8 & R4 & W4 5 & D1. 7 & B7 & No .72 & P0.16 \\
\hline 285 & L05 & \#282 & & R3 & W3 5 & D1. 1 & B3 & N0.06 & P0.01 \\
\hline 286 & L20 & $\# 282$ & $\mathrm{~A} 44$ & R8 & W4 5 & D1. 0 & B3 & No .16 & P0. 02 \\
\hline 287 & L85 & \#281 & A72 & R6 & W4 5 & D2 . 4 & B9 & N1.02 & P0. 28 \\
\hline 288 & L83 & \#281 & & R3 & W50 & D1. 7 & B6 & No. 76 & P0. 20 \\
\hline 289 & L60 & \#281 & & R1 & W30 & D1. 8 & B9 & N1. 36 & P0. 60 \\
\hline 290 & L95 & \#281 & A90 & R8 & W50 & D1. 8 & B7 & N1. 15 & P0. 27 \\
\hline 291 & L65 & \#281 & A65 & $\mathrm{R} 2$ & W4 5 & D1. 5 & B8 & N0 .54 & P0.09 \\
\hline 292 & L35 & \#281 & A10 & R1 & W4 5 & D1. 0 & B5 & N0. 18 & P0.07 \\
\hline 293 & L77 & \#9 & & R3 & W4 0 & D4 . 0 & & & \\
\hline 294 & L68 & \#293 & $\mathrm{V}$ & & & D2 . 4 & B1 & No .17 & P0. 12 \\
\hline $294 a$ & L37 & \#293 & A33 & R1 & W30 & D1. 2 & B6 & No . 40 & P0. 08 \\
\hline $294 b$ & L102 & \#293 & A4 5 & $\mathrm{R} 6$ & W30 & D3. 0 & B6 & No .73 & P0. 53 \\
\hline 295 & L34 & \#10 & A05 & R5 & W50 & D1. 8 & B7 & N0. 46 & P0.07 \\
\hline 296 & L83 & \#10 & A32 & R1 & W30 & D2. 0 & B5 & N0. 39 & P0.08 \\
\hline 297 & L8 7 & \#10 & A51 & R3 & W3 5 & D2. 0 & B2 & NO . 04 & P0.01 \\
\hline 298 & L10 & \#10 & A73 & R3 & W30 & D1. 2 & B6 & N0. 97 & P0. 48 \\
\hline
\end{tabular}

ejh:

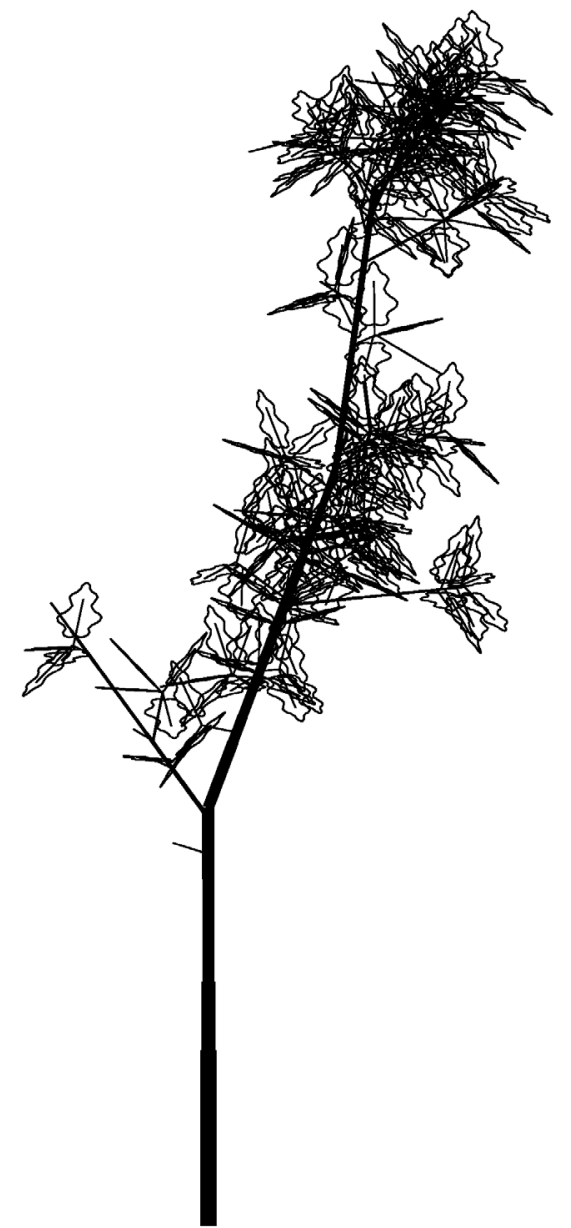

$\backslash$ leafobject eblatt.lsy veg 2,

$\backslash$ leafarea 1337,

$\backslash$ leaflength 65.9,

$\backslash$ phyllotaxy spiral, Imin_intn 10,

1 L155 \#\#

D13.4 <Basis: Ca. 17.2 oder 18.5> 


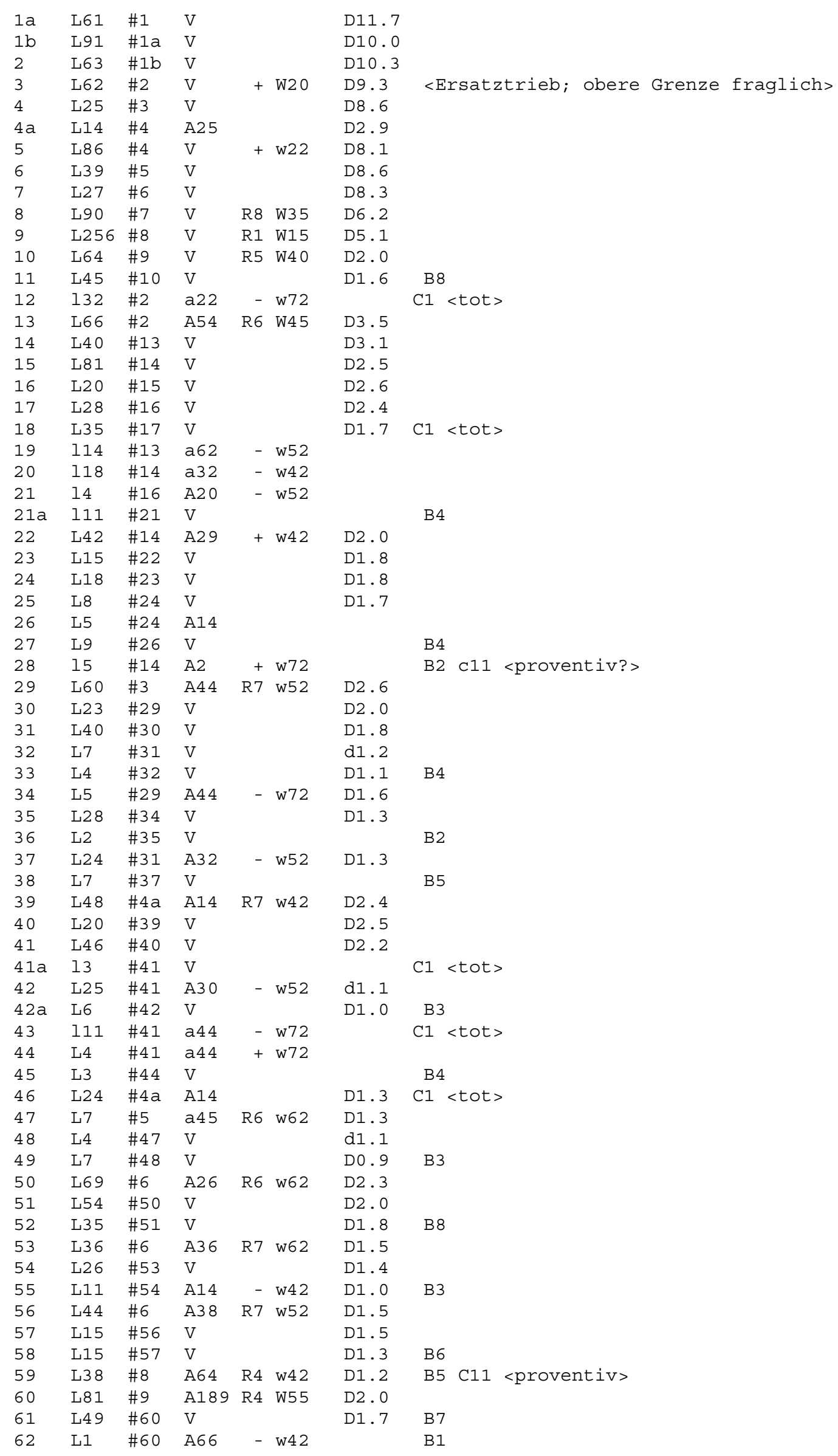




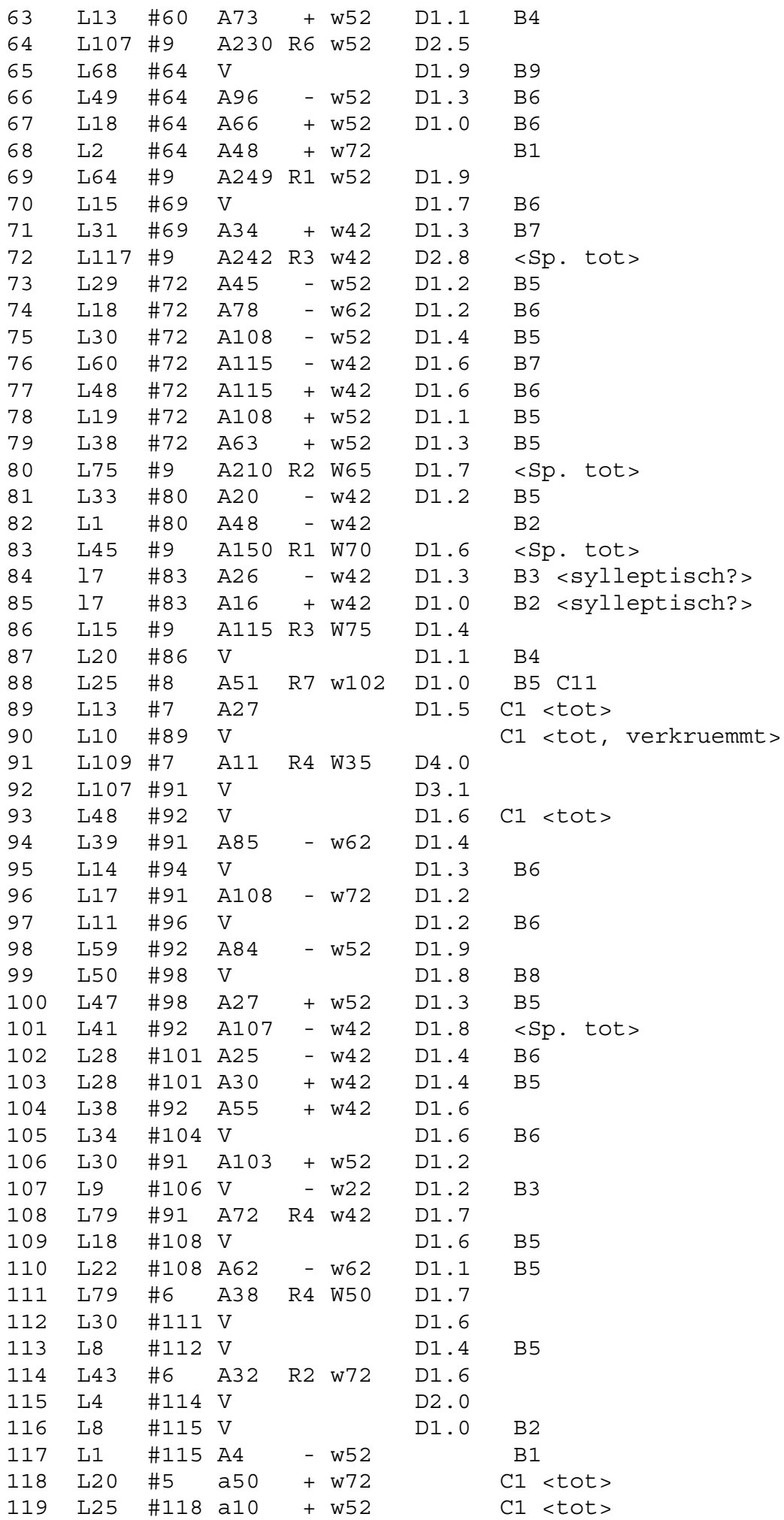


eji:

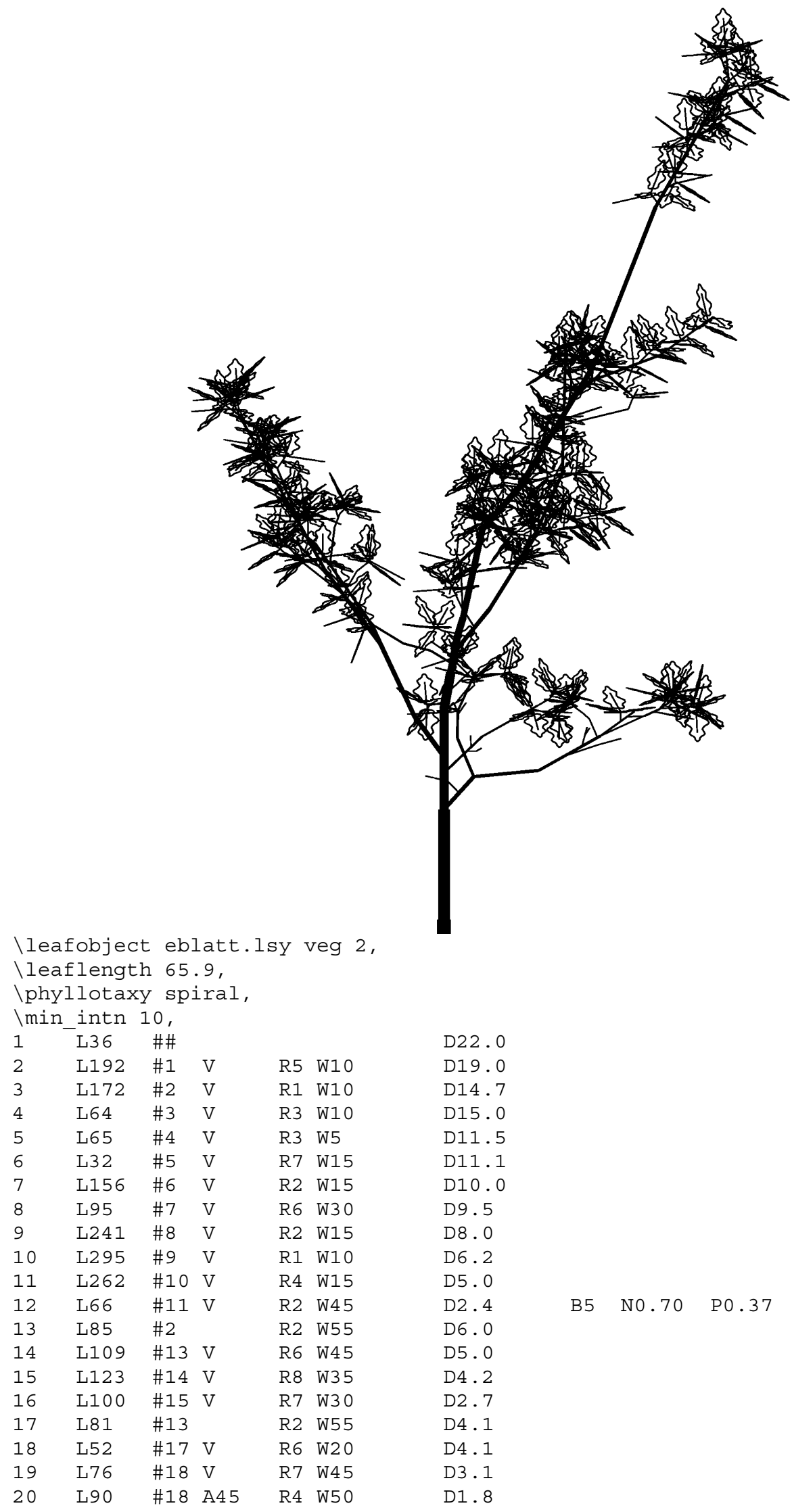




\begin{tabular}{|c|c|c|c|c|c|c|c|c|}
\hline L52 & \#19 & A5 8 & R5 & W30 & D1. 8 & & & \\
\hline L36 & \#21 & A0 5 & $\mathrm{R} 6$ & W30 & D1. 8 & & & \\
\hline L26 & \#22 & $\mathrm{V}$ & & & D2. 0 & B6 & No. 66 & P0.08 \\
\hline L2 7 & \#19 & A71 & $\mathrm{R} 2$ & W65 & D1. 8 & & & \\
\hline L15 & \#24 & $\mathrm{V}$ & & & D1. 2 & B4 & No .21 & P0.02 \\
\hline L13 & \#24 & & R1 & W4 5 & D1. 2 & B3 & No. 14 & P0.01 \\
\hline L09 & \#19 & A35 & R7 & W3 5 & D1. 7 & & & \\
\hline L16 & \#27 & $\mathrm{V}$ & & & D1. 7 & & & \\
\hline L1 7 & \#28 & $\mathrm{V}$ & $\mathrm{R} 2$ & W4 5 & D1. 4 & B5 & No. 23 & P0.01 \\
\hline L31 & \#18 & & R1 & W8 0 & D2. 1 & & & \\
\hline L15 & \#30 & $\mathrm{V}$ & R1 & W30 & $\mathrm{D} 2.2$ & & & \\
\hline L18 & \#31 & $\mathrm{V}$ & & & D2. 1 & B5 & No. 39 & P0.04 \\
\hline L26 & \#18 & & R3 & W30 & D1. 8 & & & \\
\hline L11 & \#33 & $\mathrm{V}$ & R3 & W80 & D1. 8 & & & \\
\hline L09 & \#34 & $\mathrm{V}$ & & & D1. 0 & & & \\
\hline L0 7 & \#35 & V & & & D1. 3 & B2 & No .20 & P0.02 \\
\hline L26 & \#15 & A86 & $\mathrm{R} 2$ & W70 & D1. 7 & & & \\
\hline L18 & \#37 & $\mathrm{V}$ & & & D1. 5 & & & \\
\hline L15 & \#37 & & R6 & W60 & D1. 1 & & & \\
\hline L35 & \#16 & A78 & $\mathrm{R} 4$ & W3 0 & D2. 1 & & & \\
\hline L31 & \#40 & $\mathrm{V}$ & R1 & W20 & D2. 3 & & & \\
\hline L30 & \#41 & $\mathrm{V}$ & R1 & W10 & D2. 1 & B5 & No. 68 & P0.06 \\
\hline L10 & \#16 & A60 & $\mathrm{R} 8$ & W4 0 & D1. 8 & & & \\
\hline L05 & $\# 43$ & $\mathrm{~V}$ & $\mathrm{R} 2$ & W2 0 & D1. 8 & & & \\
\hline L05 & $\# 44$ & $\mathrm{~V}$ & & & D1. 4 & B4 & No. 23 & P0.03 \\
\hline L111 & \#15 & & R3 & W3 0 & D2. 1 & & & \\
\hline L4 7 & $\# 46$ & $\mathrm{~V}$ & $\mathrm{R} 2$ & W3 5 & D2. 1 & & & \\
\hline L4 5 & $\# 46$ & & R6 & W3 5 & D1. 8 & & & \\
\hline L10 & \#48 & $\mathrm{V}$ & R3 & W4 5 & D1. 4 & & & \\
\hline L12 & \#49 & $\mathrm{V}$ & & & D1. 1 & B5 & No. 23 & P0.01 \\
\hline L25 & \#48 & A4 0 & R7 & W4 5 & D1. 8 & & & \\
\hline L15 & \#51 & $\mathrm{V}$ & & & D1. 9 & B4 & N0. 68 & P0.03 \\
\hline L08 & $\# 47$ & A35 & R6 & W4 5 & D1. 4 & B2 & No. 03 & P0.01 \\
\hline L2 5 & $\# 47$ & A41 & R3 & W4 5 & D1. 8 & & & \\
\hline L21 & \#54 & $\mathrm{V}$ & & & D1. 6 & B5 & No .46 & P0.04 \\
\hline L05 & \#4 7 & A2 0 & R1 & W4 5 & D1. 1 & & & \\
\hline L05 & \#56 & $\mathrm{V}$ & & & D1. 1 & B2 & No. 09 & P0.01 \\
\hline L0 8 & \#46 & A93 & R3 & W4 5 & D1. 7 & & & \\
\hline L05 & \#58 & $\mathrm{V}$ & & & D1. 7 & & & \\
\hline L04 & \#59 & $\mathrm{V}$ & & & D1. 4 & B1 & N0.01 & P0.02 \\
\hline L3 5 & \#15 & A117 & R1 & W85 & D1. 8 & & & \\
\hline L15 & \#61 & $\mathrm{V}$ & R1 & W2 5 & D1. 8 & & & \\
\hline L16 & \#62 & $\mathrm{V}$ & R1 & W5 & D1. 8 & & & \\
\hline L1 7 & \#63 & $\mathrm{V}$ & R5 & W5 & D1. 8 & B6 & N0. 65 & P0.04 \\
\hline L8 5 & \#15 & A51 & R3 & W60 & D1. 7 & & & \\
\hline L51 & \#65 & A8 0 & $\mathrm{R} 6$ & W5 & D1. 5 & & & \\
\hline L30 & \#13 & A43 & $\mathrm{R} 2$ & W75 & D1. 8 & & & \\
\hline L12 & \#67 & $\mathrm{V}$ & R1 & W4 5 & D1. 8 & & & \\
\hline L11 & \#68 & $\mathrm{V}$ & & & D1. 8 & & & \\
\hline L0 7 & \#69 & V & & & D1. 4 & & & \\
\hline L08 & \#70 & $\mathrm{V}$ & & & D1. 2 & & & \\
\hline L3 7 & \#3 & A63 & $\mathrm{R} 2$ & W55 & D3. 4 & & & \\
\hline L74 & \#72 & $\mathrm{V}$ & & & D3. 2 & & & \\
\hline L16 & \#73 & A2 8 & $\mathrm{R} 6$ & W4 5 & $\mathrm{D} 1.8$ & & & \\
\hline L13 & \#74 & $\mathrm{V}$ & $\mathrm{R} 2$ & W3 5 & D1. 4 & & & \\
\hline L72 & \#73 & A72 & R7 & W30 & D2. 7 & & & \\
\hline L35 & \#76 & $\mathrm{V}$ & & & D2. 7 & & & \\
\hline L22 & \#77 & $\mathrm{V}$ & & & D2. 1 & & & \\
\hline L18 & \#78 & $\mathrm{V}$ & $\mathrm{R} 2$ & W2 0 & D1. 8 & B5 & No. 75 & P0.04 \\
\hline L12 & \#77 & A11 & R7 & W60 & D1. 1 & & & \\
\hline L10 & \#80 & $\mathrm{V}$ & R1 & W30 & D1. 1 & B4 & No. 14 & P0.01 \\
\hline L2 7 & \#77 & A24 & R6 & W50 & D1. 7 & & & \\
\hline L2 0 & \#82 & $\mathrm{V}$ & & & D1. 5 & B5 & N0. 54 & P0.03 \\
\hline L46 & \#73 & A4 0 & R3 & W4 0 & D1. 7 & & & \\
\hline L86 & \#3 & A91 & $\mathrm{R} 7$ & W3 5 & D7. 4 & & & \\
\hline L177 & \#85 & $\mathrm{V}$ & $\mathrm{R} 8$ & W15 & D6. 8 & & & \\
\hline L35 & \#86 & $\mathrm{V}$ & R5 & W10 & D4. 5 & & & \\
\hline
\end{tabular}




\begin{tabular}{|c|c|c|c|c|c|c|c|c|c|}
\hline 88 & L135 & $\# 87 \mathrm{~V}$ & V & & & D3.7 & & & \\
\hline 89 & L31 & \#88 V & V & R6 & W30 & D3. 1 & & & \\
\hline 90 & L52 & $\# 89 \mathrm{~V}$ & V & R1 & W30 & D3. 8 & & & \\
\hline 91 & L65 & $\# 90 \mathrm{~V}$ & V & R2 & W30 & D2. 8 & & & \\
\hline 92 & L10 & $\# 91 \mathrm{~V}$ & V & & & D2. 6 & & & \\
\hline 93 & L54 & \#92 V & V & R4 & W4 0 & D1. 8 & B7 & N0. 97 & P0.15 \\
\hline 94 & L112 & $\# 86 \quad A$ & A135 & R3 & W30 & D5. 7 & & & \\
\hline 95 & L55 & $\# 94 \mathrm{~V}$ & V & & & D5.3 & & & \\
\hline 96 & L132 & $\# 95 \mathrm{~V}$ & V & R7 & W2 0 & D4. 6 & & & \\
\hline 97 & L85 & $\# 96 \mathrm{~V}$ & V & & & D3. 8 & & & \\
\hline 98 & L85 & \#97 V & V & & & D3. 8 & & & \\
\hline 99 & L41 & $\# 98 \mathrm{~V}$ & V & R2 & W10 & $\mathrm{D} 1.3$ & B4 & N0. 54 & P0.13 \\
\hline 100 & L92 & \#94 & & R6 & W4 0 & $\mathrm{D} 2.3$ & & & \\
\hline 101 & L4 8 & \#100 & $\mathrm{V}$ & R7 & W15 & $\mathrm{D} 2.2$ & & & \\
\hline 102 & L13 & \#101 & $\mathrm{V}$ & & & $\mathrm{D} 2.3$ & & & \\
\hline 103 & L17 & \#102 & $\mathrm{V}$ & & & $\mathrm{D} 1.3$ & B5 & No. 42 & P0.05 \\
\hline 104 & L30 & \#100 & & R3 & W2 0 & $\mathrm{D} 2.5$ & & & \\
\hline 105 & L06 & \#104 & $\mathrm{V}$ & & & D2. 0 & & & \\
\hline 106 & L12 & \#105 & $5 \mathrm{~V}$ & R2 & W4 0 & D1. 3 & B4 & No. 34 & P0.02 \\
\hline 107 & L03 & \#102 & A10 & R8 & W4 0 & D0. 9 & B4 & No. 19 & P0.02 \\
\hline 108 & L4 7 & \#96 & & $\mathrm{R} 4$ & W35 & D1. 4 & & & \\
\hline 109 & L21 & \#108 & 3 A37 & R7 & W30 & $\mathrm{D} 1.0$ & & & \\
\hline 110 & L61 & \#97 & A35 & R7 & W15 & D2. 0 & & & \\
\hline 111 & L26 & \#110 & A54 & R7 & W2 0 & $\mathrm{D} 1.2$ & B5 & No. 40 & P0.06 \\
\hline 112 & L86 & \#98 & & R7 & W50 & D1. 7 & B9 & N1.05 & P0.27 \\
\hline 113 & L52 & \#98 & & R3 & W55 & D1. 8 & B7 & No. 97 & P0.16 \\
\hline 114 & L21 & \#97 & & R8 & W35 & $\mathrm{D} 2.7$ & & & \\
\hline 115 & L32 & \#114 & $\mathrm{V}$ & & & D1. 3 & B7 & No. 79 & P0.09 \\
\hline 116 & L24 & \#97 & A09 & R3 & W60 & D1. 3 & & & \\
\hline 117 & L03 & \#116 & $5 \mathrm{~V}$ & & & D1. 8 & B2 & No. 13 & P0.01 \\
\hline 118 & L21 & \#96 & A59 & R2 & W55 & D2. 4 & & & \\
\hline 119 & L20 & \#118 & $3 \mathrm{~V}$ & & & D1. 8 & & & \\
\hline 120 & L11 & \#119 & ${ }^{3} \mathrm{~V}$ & R6 & W45 & D1. 2 & B5 & N0. 23 & P0.03 \\
\hline 121 & L121 & \#95 & A25 & R3 & W15 & D3.0 & & & \\
\hline 122 & L40 & \#121 & $-\mathrm{V}$ & $\mathrm{R} 8$ & W55 & D2. 1 & & & \\
\hline 123 & L14 & \#122 & $\mathrm{V}$ & R6 & W30 & D2. 0 & & & \\
\hline 124 & L0 4 & \#123 & $\mathrm{V}$ & & & D0. 8 & B2 & N0. 05 & P0.04 \\
\hline 125 & L16 & \#122 & A34 & R2 & W4 0 & $\mathrm{D} 1.5$ & & & \\
\hline 126 & L10 & \#125 & $5 \mathrm{~V}$ & & & D1. 1 & B5 & No. 37 & P0.01 \\
\hline 127 & L11 & \#122 & A20 & R3 & W4 0 & D1. 1 & & & \\
\hline 128 & L05 & \#127 & $\mathrm{V}$ & & & $\mathrm{D} 1.1$ & B4 & N0.09 & P0.01 \\
\hline 129 & L81 & \#121 & & R3 & W55 & D1. 8 & & & \\
\hline 130 & L34 & $\# 121$ & A74 & R4 & W50 & $\mathrm{D} 1.2$ & & & \\
\hline 131 & L15 & \#130 & $\mathrm{V}$ & & & D1.0 & & & \\
\hline 132 & L52 & \#94 & & R1 & W60 & $\mathrm{D} 1.5$ & & & \\
\hline 133 & L18 & \#132 & $\mathrm{V}$ & R1 & W2 0 & D1. 5 & & & \\
\hline $133 a$ & L05 & \#133 & $\mathrm{V}$ & & & $\mathrm{D} 1.5$ & & & \\
\hline 134 & L08 & $\# 133 a$ & $3 \mathrm{a} V$ & & & D1.0 & B3 & N0.06 & P0.01 \\
\hline 135 & L0 8 & \#133a & $a$ & R3 & W30 & D1.0 & B3 & No. 14 & P0.01 \\
\hline 136 & L72 & \#88 & A90 & R8 & W4 0 & D2. 7 & & & \\
\hline 137 & L20 & \#136 & $5 \mathrm{~V}$ & R2 & W4 5 & $\mathrm{D} 2.3$ & & & \\
\hline 138 & L06 & \#137 & $\mathrm{V}$ & R2 & W5 & $\mathrm{D} 2.2$ & & & \\
\hline 139 & L21 & \#138 & $3 \mathrm{~V}$ & R7 & W4 0 & D1.7 & B5 & N0. 58 & P0.07 \\
\hline 140 & L10 & \#137 & A18 & R5 & W4 5 & $\mathrm{D} 1.5$ & & & \\
\hline 141 & L06 & $\# 140$ & $\mathrm{~V}$ & & & $\mathrm{D} 1.3$ & B5 & No. 28 & P0.01 \\
\hline 142 & L46 & \#90 & A44 & R6 & W35 & $\mathrm{D} 1.3$ & & & \\
\hline 143 & L0 7 & $\# 142$ & $\mathrm{~V}$ & R1 & W5 & D1.1 & B3 & No. 26 & P0.10 \\
\hline 144 & L12 & \#91 & A3 7 & R8 & W30 & D0.9 & B1 & No. 10 & P0.01 \\
\hline 145 & L31 & \#92 & & R2 & W55 & D1.7 & B5 & No .64 & P0.10 \\
\hline 146 & L50 & \#92 & & R6 & W4 5 & $\mathrm{D} 1.4$ & B5 & N0. 51 & P0.10 \\
\hline 147 & L11 & \#91 & A21 & R1 & W4 5 & $\mathrm{D} 1.4$ & B2 & N0.08 & P0.01 \\
\hline 148 & L39 & \#88 & A38 & R3 & W35 & D2. 0 & & & \\
\hline 149 & L32 & \#148 & $3 \mathrm{~V}$ & R3 & W2 5 & D2. 0 & & & \\
\hline 150 & L15 & \#149 & ${ }^{3} \mathrm{~V}$ & R6 & W2 0 & D1.7 & & & \\
\hline 151 & L20 & $\# 150$ & $\mathrm{~V}$ & & & D1. 7 & B6 & N0. 48 & P0.05 \\
\hline 152 & L08 & \#149 & & R1 & W60 & D1. 8 & & & \\
\hline 153 & L16 & \#152 & $\mathrm{V}$ & & & $\mathrm{D} 1.4$ & B5 & N0. 27 & P0.02 \\
\hline
\end{tabular}




\begin{tabular}{|c|c|c|c|c|c|c|c|c|c|c|}
\hline 154 & L3 7 & \#3 & & R3 & W60 & D1. 8 & & & & \\
\hline 155 & L21 & \#154 & A23 & R2 & W30 & D1. 3 & & & & \\
\hline 156 & L1 7 & \#155 & $\mathrm{V}$ & & & D1. 3 & & & & \\
\hline 157 & L14 & $\# 4$ & A2 8 & R6 & W4 5 & D2. 1 & & & & \\
\hline 158 & L53 & \#157 & $\mathrm{V}$ & & & D2. 0 & & & & \\
\hline 159 & L87 & \#158 & V & R8 & W90 & D1. 9 & & & & \\
\hline 160 & L56 & $\# 4$ & & R3 & W55 & D7. 4 & & & & \\
\hline 161 & L15 & $\# 160$ & $\mathrm{~V}$ & R7 & W10 & D6. 8 & & & & \\
\hline 162 & L126 & \#161 & $\mathrm{V}$ & R7 & W10 & D6. 4 & & & & \\
\hline 163 & L203 & \#162 & V & R2 & W5 & D5. 7 & & & & \\
\hline 164 & L155 & $\# 163$ & V & R1 & W10 & D4. 7 & & & & \\
\hline 165 & L216 & \#164 & V & R1 & W4 0 & D4. 1 & B13 & N2. 99 & P1. 48 & \\
\hline 166 & L96 & \#162 & A54 & R8 & W60 & D2. 2 & & & & \\
\hline 167 & L4 5 & \#166 & $\mathrm{V}$ & R1 & W10 & D2. 1 & & & & \\
\hline 168 & L35 & \#167 & V & R1 & W5 & D2. 0 & B7 & N1. 11 & P0.17 & \\
\hline 169 & L18 & \#167 & A30 & R2 & W4 5 & D1. 3 & B4 & N0. 16 & P0.02 & \\
\hline 170 & L62 & $\# 163$ & A135 & R2 & W55 & D2. 1 & & & & \\
\hline 171 & L4 5 & \#170 & $\mathrm{V}$ & $\mathrm{R} 4$ & W30 & D1. 8 & B6 & N0. 83 & P0.13 & \\
\hline 172 & L68 & \#163 & A177 & R8 & W60 & D2. 8 & & & & \\
\hline 173 & L4 8 & \#172 & $\mathrm{V}$ & R5 & W30 & D2. 1 & B1 & No. 03 & P0.01 & \\
\hline 174 & L10 & \#171 & A4 3 & R2 & W30 & D1. 0 & B7 & N1.09 & P0.17 & \\
\hline 175 & L41 & \#164 & A65 & R3 & W4 5 & D1. 7 & B6 & N0. 48 & P0.09 & \\
\hline 176 & L4 7 & \#164 & $\mathrm{A} 142$ & R7 & W65 & D2. 1 & B7 & N0. 64 & P0.13 & \\
\hline 177 & L139 & \#164 & & $\mathrm{R} 4$ & W60 & D2. 4 & B8 & N1. 19 & P0.13 & \\
\hline 178 & L153 & \#163 & A152 & $\mathrm{R} 4$ & W30 & D3. 8 & & & & \\
\hline 179 & L75 & \#178 & $\mathrm{V}$ & R1 & W30 & D2. 3 & B5 & N0. 85 & P0. 20 & \\
\hline 180 & L2 8 & \#178 & A122 & R8 & W65 & D1. 6 & B5 & No .47 & P0.07 & \\
\hline 181 & L81 & \#178 & A138 & R7 & W4 5 & D2. 0 & B7 & No. 79 & P0. 21 & \\
\hline 182 & L5 8 & \#178 & A149 & R3 & W4 5 & D1. 8 & B6 & N0. 92 & P0.16 & \\
\hline 183 & L4 5 & $\# 162$ & A69 & R2 & W60 & D2. 2 & & & & \\
\hline 184 & L2 0 & \#183 & $\mathrm{V}$ & R5 & W15 & D1. 8 & & & & \\
\hline 185 & L3 8 & \#184 & $\mathrm{V}$ & & & D1. 8 & B7 & N0. 87 & P0.09 & \\
\hline 186 & L39 & \#162 & A4 0 & R3 & W70 & D1. 7 & & & & \\
\hline 187 & L21 & \#186 & $\mathrm{V}$ & R5 & W5 & D1. 4 & & & & \\
\hline 188 & L23 & \#187 & $\mathrm{V}$ & R1 & W15 & D1. 4 & B5 & N0. 35 & P0.06 & \\
\hline 189 & L56 & $\# 160$ & A2 7 & R3 & W4 5 & D3. 1 & & & & \\
\hline 190 & L53 & \#189 & $\mathrm{V}$ & R3 & W2 0 & D3. 2 & & & & \\
\hline 191 & L81 & \#190 & $\mathrm{V}$ & R7 & W2 0 & D3. 0 & & & & \\
\hline 192 & L98 & \#191 & $\mathrm{V}$ & R1 & W2 0 & D2. 4 & B8 & N1. 72 & P0. 43 & \\
\hline 193 & L03 & \#191 & A79 & R8 & W30 & D0. 3 & & & & \\
\hline 194 & L24 & \#191 & A70 & R3 & W4 5 & D1. 5 & B4 & No .25 & P0.03 & \\
\hline 195 & L90 & \#6 & & R7 & W3 5 & D2. 3 & & & & \\
\hline 196 & L4 2 & \#195 & $\mathrm{V}$ & R6 & W5 & D2. 2 & & & & \\
\hline 197 & L34 & \#196 & A37 & R6 & W10 & D1. 8 & & & & \\
\hline 198 & L34 & \#196 & A14 & R6 & W10 & D1. 7 & B6 & N0. 62 & P0. 02 & \\
\hline 199 & L06 & \#195 & & R2 & W4 0 & D1. 9 & & & & C8 \\
\hline 200 & L86 & $\# 7$ & A135 & R3 & W4 5 & D2. 8 & & & & \\
\hline 201 & L35 & $\# 200$ & $\mathrm{~V}$ & $\mathrm{R} 4$ & W4 0 & D1. 8 & & & & \\
\hline 202 & L2 7 & $\# 200$ & & R7 & W3 5 & D1.9 & & & & \\
\hline 203 & L15 & \#202 & $\mathrm{V}$ & R5 & W4 0 & D2. 1 & & & & \\
\hline 204 & L10 & \#203 & $\mathrm{V}$ & R1 & W2 0 & D1. 7 & B5 & No. 74 & P0.09 & \\
\hline 205 & L1 7 & \#201 & A05 & R7 & W60 & D1. 1 & & & & \\
\hline 206 & L07 & \#205 & $\mathrm{V}$ & & & D1. 0 & B3 & N0.16 & P0.01 & \\
\hline 207 & L07 & \#205 & A15 & R1 & W30 & D1. 1 & & & & C8 \\
\hline 208 & L 81 & \#8 & A53 & $\mathrm{R} 4$ & W70 & D2. 5 & & & & \\
\hline 209 & L63 & \#208 & $\mathrm{V}$ & & & D2. 0 & & & & \\
\hline 210 & L61 & \#209 & $\mathrm{V}$ & R1 & W2 0 & D1. 8 & B6 & N1. 36 & P0. 12 & \\
\hline 211 & L12 & \#208 & A53 & R1 & W50 & D1. 4 & B1 & N0.03 & P0.03 & \\
\hline 212 & L11 & \#209 & A33 & R8 & W80 & D1. 0 & B2 & N0.05 & P0.01 & \\
\hline 213 & L14 & \#209 & & R3 & W4 5 & D0. 9 & B2 & No. 09 & P0. 02 & \\
\hline 214 & L2 0 & \#208 & A2 6 & R6 & W80 & D0.9 & & & & \\
\hline 215 & L126 & \#8 & A74 & R7 & W4 5 & D4. 5 & & & & \\
\hline 216 & L118 & \#215 & V & R7 & W15 & D4. 9 & & & & \\
\hline 217 & L70 & \#216 & A2 5 & R6 & W30 & D1. 8 & B6 & N0. 80 & P0.16 & \\
\hline 218 & L26 & \#216 & A10 07 & R2 & W50 & D2. 2 & B6 & N0. 97 & P0. 06 & \\
\hline 219 & L26 & \#216 & A4 2 & R2 & W50 & D1. 8 & B5 & N0. 52 & P0.05 & \\
\hline 220 & L11 & \#115 & A2 7 & R7 & W55 & D1. 4 & & & & \\
\hline
\end{tabular}




\begin{tabular}{|c|c|c|c|c|c|c|c|c|c|}
\hline 221 & L 21 & \#220 & $\mathrm{V}$ & R1 & W2 0 & D1.3 & & & \\
\hline 222 & L71 & \#8 & A53 & R2 & W80 & D2. 7 & & & \\
\hline 223 & L32 & \#222 & V & R3 & W4 5 & $\mathrm{D} 1.4$ & B5 & N0. 57 & P0.07 \\
\hline 224 & L35 & \#222 & A 45 & R7 & W4 5 & $\mathrm{D} 2.0$ & B4 & N0. 33 & P0.03 \\
\hline 225 & L53 & \#224 & V & R3 & W35 & D1.9 & B5 & N0.81 & P0.12 \\
\hline 226 & L14 & \#224 & & R1 & W65 & $\mathrm{D} 1.3$ & B5 & N0. 31 & P0.02 \\
\hline 227 & L39 & \#224 & & R7 & W60 & $\mathrm{D} 1.4$ & B4 & No. 44 & P0.07 \\
\hline 228 & L126 & \#9 & $\mathrm{A} 122$ & R5 & W4 5 & $\mathrm{D} 2.3$ & & & \\
\hline 229 & L25 & \#228 & V & R1 & W55 & D1. 3 & B4 & No. 44 & P0.04 \\
\hline 230 & L105 & \#9 & A162 & R3 & W60 & D3.0 & & & \\
\hline 231 & L81 & \#230 & V & R2 & W15 & D2. 8 & B7 & N2. 15 & P0.45 \\
\hline 232 & L131 & \#9 & A176 & R7 & W55 & $\mathrm{D} 2.5$ & & & \\
\hline 233 & L07 & \#232 & A52 & R3 & W4 5 & D2. 4 & B2 & N0.06 & P0.01 \\
\hline 234 & L22 & \#232 & A98 & R2 & W4 5 & $\mathrm{D} 1.8$ & B6 & N0. 60 & P0.05 \\
\hline 235 & L11 & \#232 & A70 & R7 & W4 5 & D1.0 & B4 & No. 40 & P0.05 \\
\hline 236 & L56 & \#9 & A192 & R2 & W60 & D1. 8 & & & \\
\hline 237 & L13 & \#236 & V & R4 & W30 & $\mathrm{D} 1.2$ & B4 & N0. 25 & P0.02 \\
\hline 238 & L94 & \#9 & A2 06 & R8 & W50 & D2. 0 & & & \\
\hline 239 & L12 & \#238 & A85 & R7 & W50 & D1. 8 & B5 & N0. 64 & P0.06 \\
\hline 240 & L18 & \#238 & A78 & R3 & W4 5 & D1. 5 & B4 & No. 23 & P0.01 \\
\hline 241 & L12 & \#238 & A31 & R2 & W50 & D1. 3 & B5 & No. 20 & P0.05 \\
\hline 242 & L143 & \#9 & A2 07 & R6 & W4 5 & D3. 8 & & & \\
\hline 243 & L115 & \#242 & V & R1 & W15 & D2. 7 & B9 & $\mathrm{N} 2.55$ & P0. 63 \\
\hline 244 & L24 & \#242 & A85 & R7 & W45 & D1. 4 & B3 & N0. 27 & P0.05 \\
\hline 245 & L50 & \#242 & A104 & R2 & W4 0 & D1. 7 & B7 & N0. 65 & P0.11 \\
\hline 246 & L25 & \#11 & A0 8 & R2 & W60 & D1. 5 & B3 & No. 20 & P0.02 \\
\hline 247 & L17 & \#11 & A2 0 & R6 & W4 0 & D1. 4 & B1 & N0.13 & P0.03 \\
\hline 248 & L44 & \#11 & A39 & R4 & W50 & D1. 5 & B4 & N0. 38 & P0.09 \\
\hline 249 & L14 & \#11 & A59 & R8 & W45 & D1. 5 & B3 & N0. 29 & P0.03 \\
\hline 250 & L54 & \#11 & A113 & R3 & W4 0 & D2. 1 & B8 & $\mathrm{N} 1.12$ & P0.19 \\
\hline 251 & L49 & \#11 & $\mathrm{A} 144$ & R7 & W55 & D1. 8 & B5 & N0. 67 & P0.18 \\
\hline 252 & L8 7 & \#11 & A168 & R4 & W50 & D1.9 & B7 & $\mathrm{N} 1.03$ & P0.27 \\
\hline 253 & L25 & \#11 & A190 & R1 & W4 5 & D2. 1 & B5 & N0.90 & P0.10 \\
\hline 254 & L81 & \#11 & A2 21 & R5 & W35 & D2. 1 & B6 & N0.88 & P0.20 \\
\hline 255 & L4 1 & \#11 & A258 & R7 & W4 5 & D2. 1 & B5 & No. 70 & P0.22 \\
\hline 256 & L112 & \#11 & & R3 & W4 5 & $\mathrm{D} 2.2$ & B5 & N0. 62 & P0.60 \\
\hline
\end{tabular}

ejk :

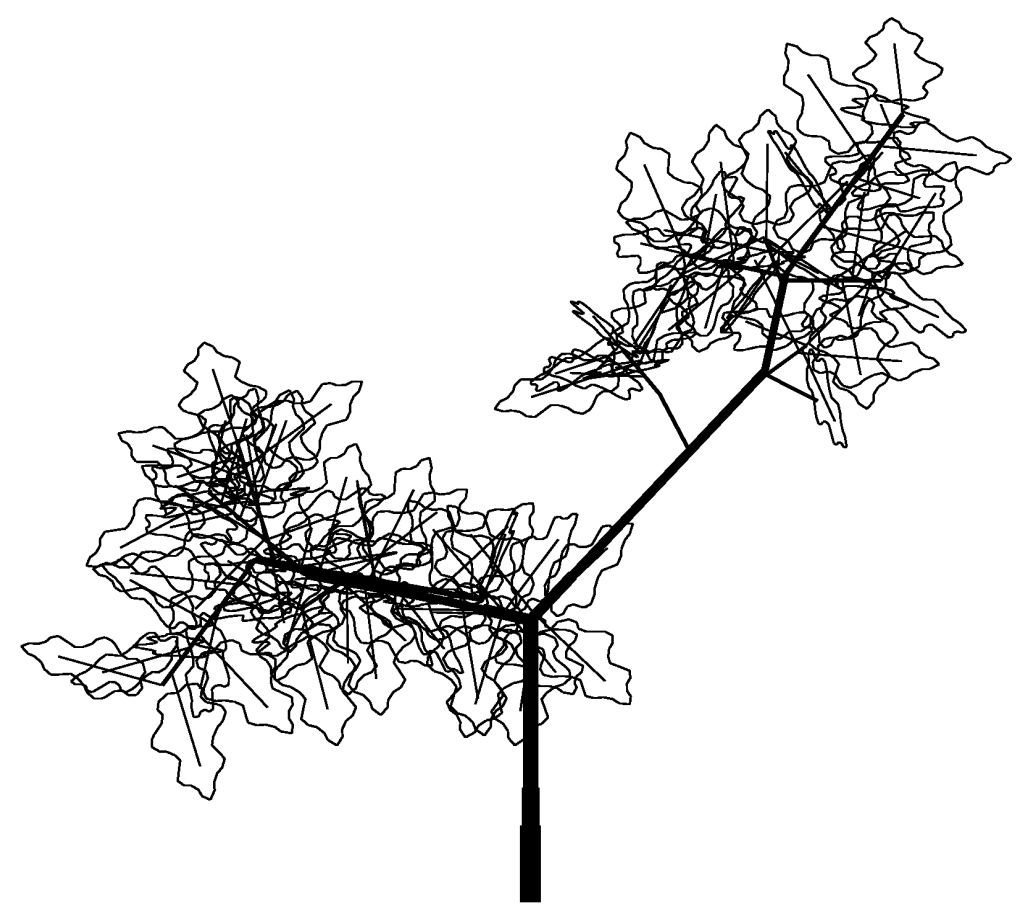




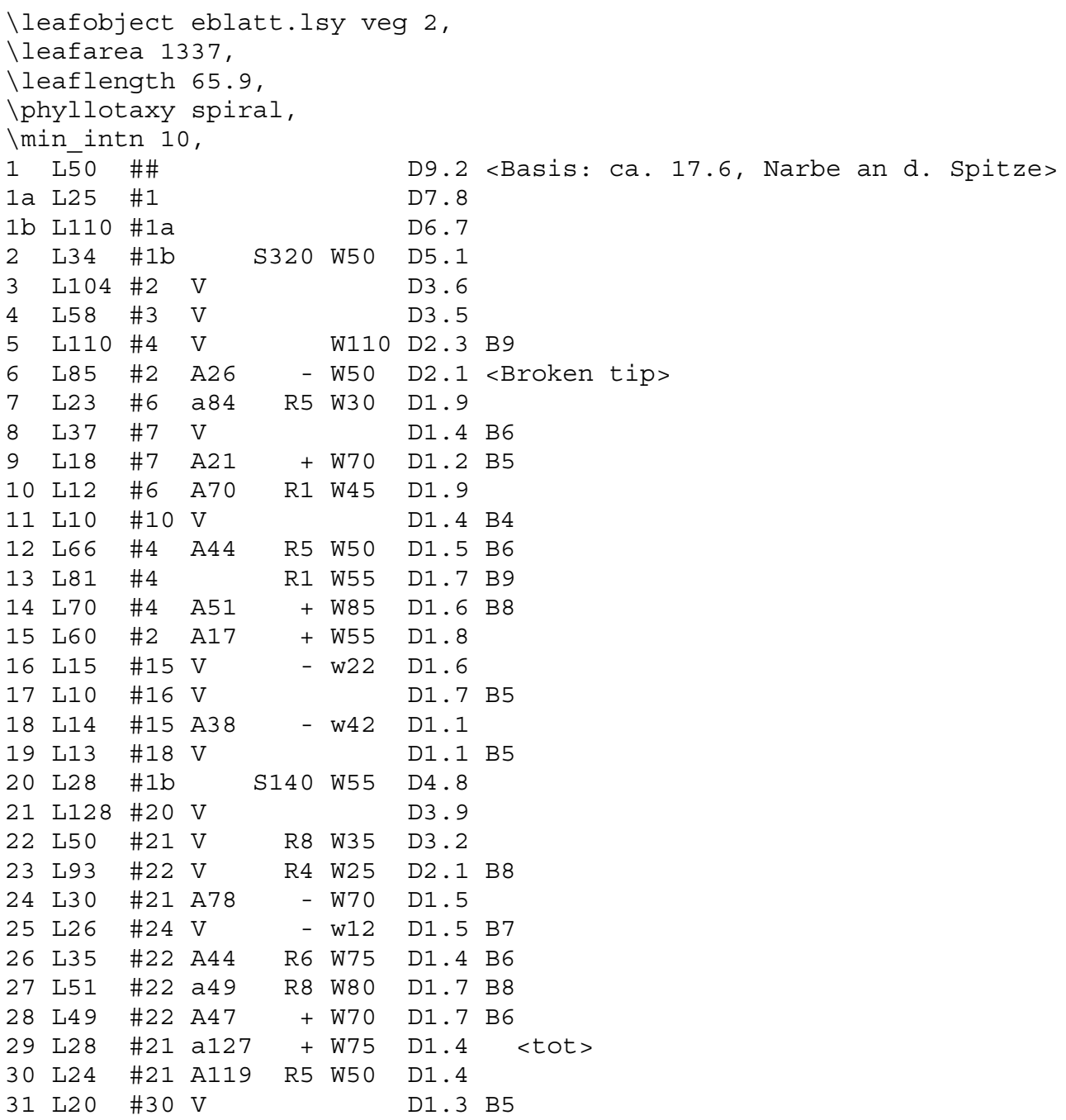




\section{APPENDIX 2}

The listing of the file epi2cdi2.ssy used to demonstrate the connection GROGRA - NEXUS.

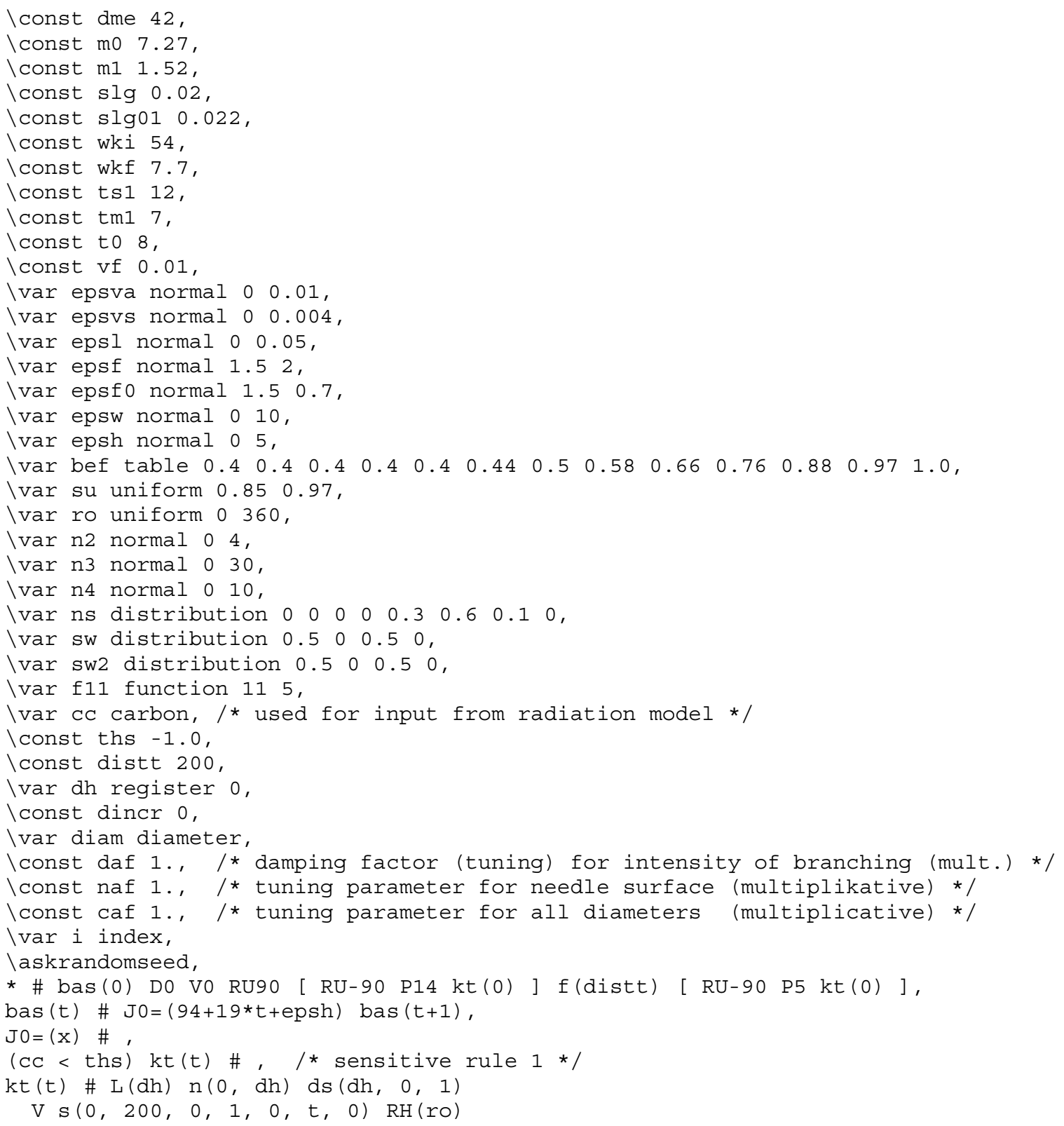




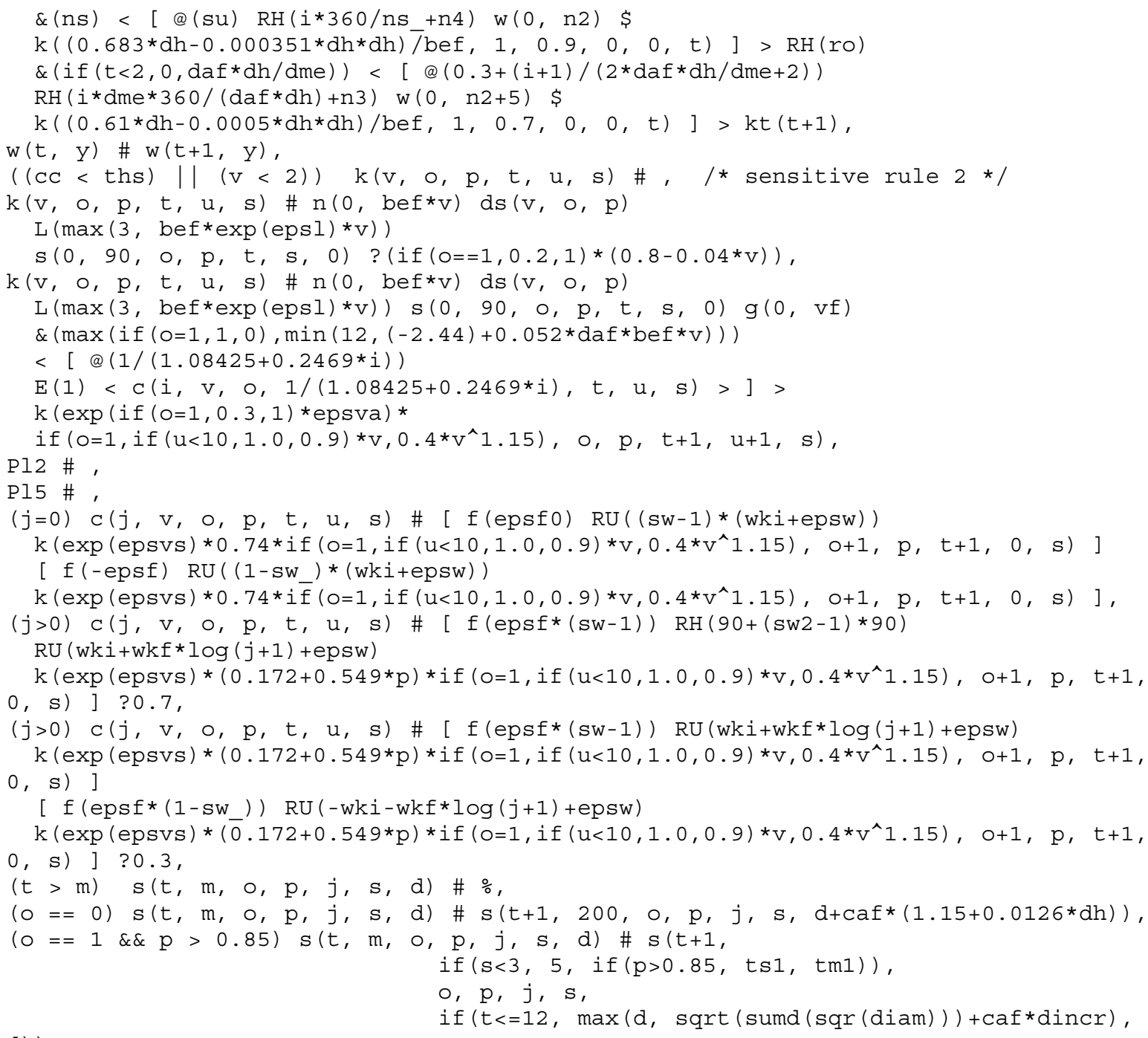

d) ),

$(0==1 \& \& p<=0.85) \mathrm{s}(t, \mathrm{~m}, \mathrm{o}, \mathrm{p}, \mathrm{j}, \mathrm{s}, \mathrm{d}) \# \mathrm{~s}(\mathrm{t}+1$, if $(s<3,5$, if $(p>0.85, t s 1, t m 1))$, $0, p, j, s$, if $(t<=4, \max (d$,

sqrt (sumd (sqr (diam))) +0.7* caf*dincr), d)),

$(0==2) s(t, m, o, p, j, s, d) \# s(t+1, t 0,0, p, j, s$, if $(t<=6, \max (d$,

sqrt (sumd (sqr (diam)) )+0.7*caf*dincr), d)),

$(o>2) \quad s(t, m, o, p, j, s, d) \# s(t+1, t 0, o, p, j, s$, if $(t<=2, \max (d$,

sqrt (sumd (sqr (diam))) +0.5*caf*dincr), d)),

$\mathrm{n}(\mathrm{t}, \mathrm{v}) \# \mathrm{n}(\mathrm{t}+1, \mathrm{v})$,

$g(t, s) \# g(t+1, s)$,

$w(t, y) \# R U(\operatorname{atg}(0.19 *(t-3)) / 3+60+y)$,

$(t<=11) \mathrm{n}(\mathrm{t}, \mathrm{v})$ \#\# $\mathrm{N}((\operatorname{naf} * \mathrm{~m} 0 * \mathrm{v} \wedge \mathrm{m} 1) * i f(t<=3,1,(11-t) / 8))$,

$(t>11) \mathrm{n}(t, v)$ \#\# No,

$\mathrm{ds}(\mathrm{v}, \mathrm{o}, \mathrm{p})$ \#\# D(if $(\mathrm{o}<=1, \mathrm{~s} \lg 01, \mathrm{slg})$ * caf * v),

$g(t, s)$ \#\# $V(s *(t * t-2 * t+1))$,

$(\mathrm{o}==1 \& \& \mathrm{p}>0.85) \quad \mathrm{s}(\mathrm{t}, \mathrm{m}, \mathrm{o}, \mathrm{p}, \mathrm{k}, \mathrm{s}, \mathrm{d}) \# \# \mathrm{Dl}+(\mathrm{d})$

$\mathrm{V}\left(\mathrm{f} 11\left(k, t, 2.5 /(k+t+10)^{\wedge} 2,6 /(k+t+10)^{\wedge} 2,0.3-0.015 * \operatorname{sqrt}(k+t)\right)\right) \quad R V F$,

$(0==1 \& \& \mathrm{p}<=0.85) \mathrm{s}(\mathrm{t}, \mathrm{m}, \mathrm{o}, \mathrm{p}, \mathrm{k}, \mathrm{s}, \mathrm{d}) \# \# \mathrm{Dl}+(\mathrm{d})$

$\mathrm{V}(\mathrm{f} 11(k, t, 0.01,0.01,0.25)) \mathrm{RV} \mathrm{F}$,

$\mathrm{s}(\mathrm{t}, \mathrm{m}, \mathrm{o}, \mathrm{p}, \mathrm{k}, \mathrm{s}, \mathrm{d})$ \#\# $\mathrm{Dl}+(\mathrm{d}) \mathrm{RV} F$ 


\section{CURRICULUM Vitae}

\section{Personal Data}

Born

Marital status

Parents

\section{School}

1978-1983 primary school

1983-1985 primary school

1985-1986 high school

1986-1987 high school

1987-1990 high school

July $18^{\text {th }}, 1990$

\section{Preparation for studies}

Nov. $2^{\text {nd }}, 1990$ - May $31^{\text {st }}, 1991$

June $16^{\text {th }}, 1991-$ Aug. $31^{\text {st }}, 1991$

Sep. $14^{\text {th }}, 1991-$ June $25^{\text {th }}, 1992$

\section{Study}

Oct. 1992 - March 1993

Oct. 1992 - Aug. 1998

March 21 ${ }^{\text {st }}, 1995$

Aug. $17^{\text {th }}, 1998$

\section{Occupations}

Sept. $04^{\text {th }}-22^{\text {nd }}, 1995$;

Nov. $1^{\text {st }}-31^{\text {st }}, 1995$;

Oct. $1^{\text {st }}-10^{\text {th }}, 1996$;

Sept. $21^{\text {st }}-25^{\text {th }}, 1997$;

Nov. $14^{\text {th }}, 1997$ - Dec. $9^{\text {th }}, 1997$;

Dec. $22^{\text {nd }}, 1997$ - Jan. $19^{\text {th }}, 1998$;

Apr. $1^{\text {st }}-24^{\text {th }}, 1998$

Nov. $1^{\text {st }}, 1998-$ Apr. $19^{\text {th }}, 2002$
Oct. $1^{\text {st }}, 1972$ in Valera, Venezuela

Unmarried

Dr. Med. Héctor L. Anzola Guerra

Dr. Med. Ingrid Jürgenson Tamsalu

"Instituto de Educación Integral", Caracas, Venezuela

"Colegio El Mundo de Los Niños", Cabimas, Venezuela

"Colegio El Mundo de Los Niños", Cabimas, Venezuela

"Colegio Nuestra Madre", Caracas, Venezuela

"Colegio Ignacio Martin Burg", Valera, Venezuela

Graduation in sciences and mathematics

Classes in German language at the Carl Duisberg Centren (CDC), in Cologne und Radolfzell am Bodensee, Germany

Practical course at the Forest Administration Office of Seesen, Germany

German High School grade obtained at the Niedersächsisches Studienkolleg of the University Hannover, Germany

Practical course at the Forest Administration Office of Bovenden, Germany

Studies of forest sciences, with the specialisation in forest ecology at the Georg-August-University in Göttingen, Germany

Pre-degree examination in Forestry

Graduation as Diplom Forstwirt with the graduation thesis: "Analysis and Modeling of the Morphology of young spruce trees (Picea abies) in dependence of light and growth density" (in German)

Periods of employment as research assistant at the Institute for Forest Biometry and Applied Computer Science of the University of Göttingen, Germany

Employment as researcher at the Institute for Forest Biometry and Applied Computer Science of the University of Göttingen, Germany 